R. R. Danie1

Tata Institute of Fundamental Research Homi Bhabha Road, Bombay, India

\author{
and \\ S. A. Stephens* \\ Department of Physics Astronomy, University of Maryland \\ and \\ Goddard Space Flight Center, Greenbelt, Maryland, U.S.A.
}

Technical Report No. 75-028

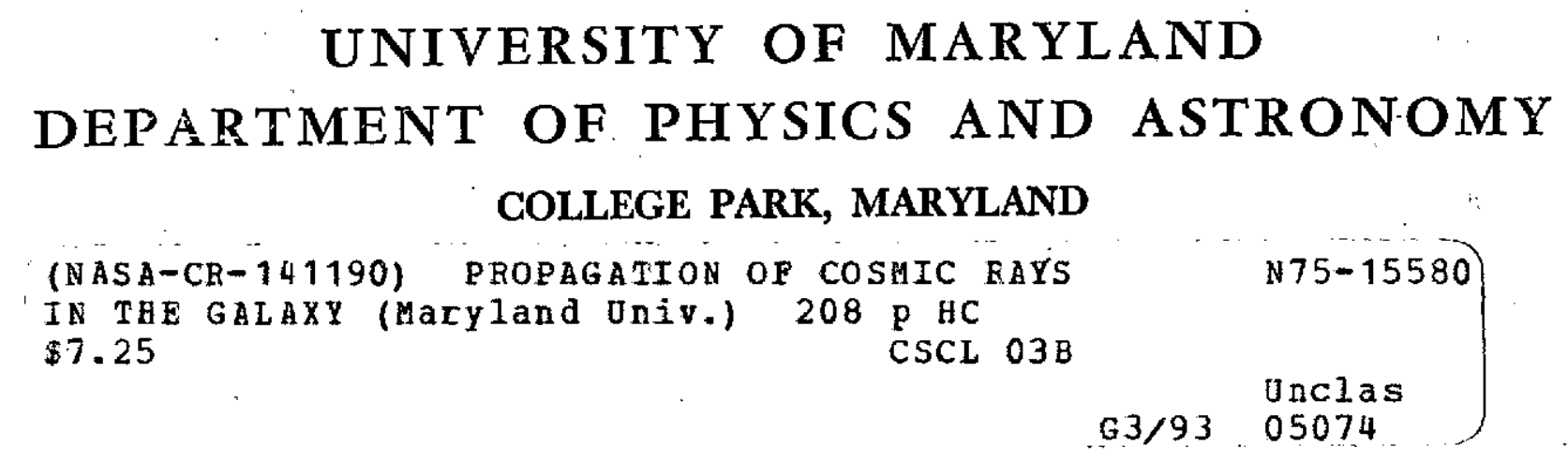


This is a preprint of research carried out at the University of Maryland. In order to promote the active exchange of research results, individuals and groups at your institution are encouraged to send their preprints to

PREPRINT LIBRARY

DEPARTMENT 'OF PHYSICS AND ASTRONOMY

UNIVERSITY OF MARYLAND

COLLEGE PARK, MARYLAND

20742

U.S.A. 


\title{
PROPAGATION OF COSMIC RAYS IN THE GALAXY
}

\author{
R. R. Daniel \\ Tata Institute of Fundamental Research \\ Homi Bhabha Road, Bombay, India \\ and \\ S. A. Stephens* \\ Department of Physics \& Astronomy, University of Maryland \\ and \\ Goddard Space Flight Center, Greenbelt, Maryland, U.S.A.
}

Technical Report No. 75-028

To appear in Space Science Reviews

*on leave from Tata Institute of Fundamental Research, Bombay, India. 
CONTENTS

1. Introduction

1.1. Models of cosmic ray confinement

1.2. The galactic model for cosmic ray confinement

1.3. Scope of the present review

2. General description of the Galaxy

2.1. Dimensions and density

2.2. Structure

2.3. Interstellar gas

2.4. Magnetic fields

2.5. Radiation fields

3. Cosmic ray propagation in the Galaxy: Theoretical aspects

3.1. Quasi-stationary state and spatial homogenity of cosmic rays

3.2. Models of diffusion of cosmic rays

3.2.1. Motion of charged particles in a magnetic field and their diffusion

3.2.2. Self-scattering of cosmic rays

3.2.3. Instability model

3.3. Diffusion process and isotropy of cosmic rays

3.4. General formulation of the propagation of cosmic rays in interstellar space

3.5. Quasi-equilibrium models

3.5.1. Nucleonic components

3.5.2. Electronic components 
4. Interpretation of the observed data

4.1. Matter traversed by cosmlc rays

4.1.1. The effect of ionization

4.1.2. Fragmentation of nuclei

4.1.3. Charge composition of cosmic ray electrons

4.1.4. Gamma-ray component of the galactic background radiation

4.1.5. Sumnary

4.2. The chemical composition at the source

4.3. Modification of energy spectrum at low energies

4.3.1. L/M ratio

4.3.2. ${ }^{2} \mathrm{H} /{ }^{4} \mathrm{He}$ ratio

4.3.3. ${ }^{3} \mathrm{He} /{ }^{4} \mathrm{He}$ ratio

4.3.4. He/M rat1o

4.3.5. He/LH ratio

4.3.6. He/VH ratio

4.3.7. Summary

4.4. Modification of energy spectrum at high energies

4.4.1. Observed change in the abundances of secondary nuclei

4.4.2. Energy dependence in the abundance of primary nuclei

4.4.3. Theoretical models to explain the change in composition

4.5. Propagation of the electron component and its source characteristics

4.5.1. Secondary electrons in the Galaxy

4.5.2. Directly accelerated electrons

4.5.3. The origin of cosmic ray electrons

4.5.4. Interpretation of high energy electron spectrum 
4.6. Leakage lifetime of cosmic rays

4.6.1. Mean matter traversed by cosmic rays and the residence time

4.6.2. Leakage 1 ifetime from long-lived radio nuclei in cosmic rays

4.7. Anti-nuclei in cosmic rays

4.7.1. Anti-nuclet of secondary origin

4.7.2. Ant1-nuclet of primary origin

5. Cosmogentc electromagnetic radiations in the Galaxy

5.1. Cosmic ray interaction with interstellar gas

5.1.1. Pionic gamma rays

5.1.2. Bremsstrahlung radiation

5.2. Cosmic ray interaction with radiation fields

5.3. Synchrotron radiation

5.3.1. Observational data

5.3.2. The energy spectrum of electrons in interstellar space

5.3.3. Magnetic fields in the Galaxy

5.3.4. Mean density of cosmic rays in different regions of the Galaxy

5.3.5. Some inferences on the properties of interstellar medium

5.3.6. The galactic halo

6. The role of cosmic rays in galactic dynamics

6.1. Hydrostatic equilibrium of the gaseous component of the Galaxy

6.2. Stability of self-gravitating gas and the formation of clouds

6.3. Thermal equilibrium of interstellar gas 


\section{Introduction}

As early as the mid-nineteen twenties, when scientists were fust beginning to accept the concept of the extraterrestrial origin of the radiation which discharged electroscopes carried aloft in manned ba11oons, and even when knowledge of the cosmos was still quite vague and unsettled, James Jeans (1925) speculated that the newly discovered cosmic radiation filled the entire universe. After two decades and a half, Rtchtmeyer and Teller (1949) proposed the other extreme kind of model wherein cosmic rays are supposed to be confined to the solar neighborhood; this model was masterfully developed later by Alfven (1949, 1950) when little was yet known of the nature of this radiation. Between then and now, there has been a spate of astounding and far reaching observational information on cosmic rays and astronomy. This in turn has stimulated the proposal and development of a variety of models and theories for the confinement and propagation of cosmic rays.

For any model and theory of propagation of cosmic rays to be acceptable, it should be able, among other things, to explain satisfactorily the relevant observational data on cosmic rays. These include: (i) the chemical and isotopic composition of cosmic rays as a function of energy; (ii) the flux and energy spectrum of the individual nucleonic components; (iii) the flux and energy spectrum of the electronic component; (iv) the cosmic ray prehistory; and ( $v$ ) the degree of isotropy in their arrival directions as a function of energy. The model should also be able to lead naturally and quantitatively to the observed intensities and spectra of electromagnetic radiations arising from interactions of cosmic rays with particles and fields in the space traversed by them such as pionic gamma rays from 
nuclear interactions of cosmic rays with matter and synchrotron radiation emitted by electrons spiralling along the feeble magnetic fields. As at present the model which has been able to bring to pass the greatest measure of success in this respect is the galactic confinement model. Proposals have also been made that cosmic rays may be confined to a cluster of galaxies of which our own, the Milky Way, is a member.

\subsection{Models of Cosmic Ray Confinement}

of the various possibilities listed above, the solar model can be ruled out as untenable because of the many insuperable obstacles it encounters. Among others they include: (i) the anticorrelation between the cosmic ray intensity observed near the Earth and the 11-year solar activity cycle; (ii) the disagreement between the elemental abundance of cosmic rays and the solar composition; (iii) the significant difference between the steep spectral shape of particles emitted at times of solar flares and the relatively flat energy spectrum associated with cosmic rays at quiet times; (iv) the existence in cosmic rays of a continuation of the power law energy spectrum from a few $\mathrm{GeV}$ up to $10^{12} \mathrm{eV}$ and extending even many orders beyond; (v) the difficulty of explaining simultaneously the few g. $\mathrm{cm}^{-2}$ of matter that cosmic rays are known to have traversed before reaching us and the spectral shape of electrons; and (vi) the fact that we know from studies of the diffuse galactic radio noise, that the intensity of energetic cosmic ray electrons in interstellar space is of the same magnitude as is sampled near the Earth.

Under extragalactic confinement models there are three important variations: (a) the universal mode1 (Jeans, 1925; Lamaitre, 1931; Burbidge and 
Hoyle, 1964); (b) the super cluster model (Burbidge, 1962); and (c) the local cluster model (Sciama, 1962). By their very nature these models are associated with considerable amount of speculation arising from the severe lack of information on objects and space beyond our galaxy. On the other hand these models derive their strength from recent observations on the frequent occurrence of violent extragalactic events releasing colossal amounts of energy in energet1c particles and hard quanta. The most important arguments against extragalactic models are the following: (i) The flux of hard quanta expected to result from the inverse Compton scattering of cosmic ray electrons residing within the confinement region (having an intensity same as in near-interstellar space), with photons associated with visible light and the universal black body radiation at $2.7^{\circ} \mathrm{K}$, can be calculated as a function of the dimension of the confinement region. This can then be compared with the total observed flux of the isotropic component of gamma rays of energy in excess of $100 \mathrm{MeV}$ (Fichtel et al., 1973; Kraushaar et al., 1972) to set an upper limit for the radiating distance which is found to be only $\sim 10^{23} \mathrm{~cm}$ (Daniel and Stephens, 1970); this dimension may be compared with the diameter of our own galaxy which is $210^{23} \mathrm{~cm}$, the dimension of $\sim 10^{24} \mathrm{~cm}$ for the local cluster of galaxies and of $\sim 10^{27}$ cm for the supercluster. (ii) In a similar fashion if one attributes the entire flux of the observed isotropic X-rays (Metzger et a1., 1964) to the inverse Compton scattering of electrons filling all space, with photons of the universal black body radiation (Felten and Morrison, 1966), one notes that the flux of electrons needed is a factor of 40 or perhaps even $10^{3}$ smaller (Longair, 1970) than what is known to exist in interstellar space. (1ii) A study of the relative intensities of Be and $B$ nuclei 
(Brown et al., 1973a; O'Dell et al., 1973; Webber et al., 1973a) and that of the spectral shape of cosmic ray electrons (Daniel and Stephens, 1967) strongly suggest that the residence time of cosmic rays in the storage space is legs than $10^{7}$ years whereas typical time scales involved in extragalactic models is about two orders of magnttude larger. (iv) From a detalled examination of the energetics involved in extragalactic models, Ginzburg and Syrovatski1 $(1964,1966,1968)$ have come to the conclusion that the energy density of cosmic rays in metagalactic space is likely to be exceedingly small compared to that in our galaxy. In spite of all this, recently there have been attempts for the revival of the extragalactic model for cosmic rays though not on an universal scale (Setti and Woltjer, 1971 ; Brecher and Burbidge, 1972).

It is thus seen from the above that one is able to provide decisive arguments to reject the solar model of confinement of cosmic rays. On the other hand, though the situation regarding extragalactic models, particularly the cluster models, is not that decisive, the many individual arguments add up and pose forclble enough objections to consider them, if at all, as poor substitutes. This leaves only the Galactic Model for serlous consideration for the propagation and confinement of cosmic rays, a model that has been able to explain eminently well all the observations so far available, and one which has found acceptance from the widest circle of cosmic ray physicists and astrophysicists; it is in brief the best working hypothesis we have at present.

1.2 The Galactic Model for Cosmic Ray Confinement:

The most direct evidence for cosmic rays pervading the entire galactic space stems from the observation of the diffuse galactic radio noise which 
is now untversally accepted as due to synchrotron radiation emitted by cosmfc ray electrons spiralling along the weak interstellar magnetic field lines; if there are electrons, there is no reason to doubt that energetic nuclei also exist therein. We also have equally strong evidence to demonstrate that estimates on the energetics and number densities of potential cosmic ray sources such as super novae and pulsars in the Galaxy are adequate to explain the cosmic ray intensities, as also its other features observed near the Earth. All these suggest a simple model in which cosmlc rays are generated in galactic objects and injected into interstellar space; this radiation then propagates in the Galaxy until individual particles are removed from the beam through catastrophic processes or their leakage out of the Galaxy. While traversing galactic space, these particles undergo a variety of modifications, further to those which would depend crucially on the properties of interstellar medium such as matter, magnetic field and radiation field existing therein. Thus a careful and detafled study of cosmic rays reaching the vicinity of the Earth would, in principle, be capable of revealing some of them.

The early observations on the diffuse galactic radio noise seemed to suggest that the emitting region had a quast-spherical shape consisting of an intensely radto emitting Disk encompassed by a weakly radiating Halo (Shklovsky, 1952; Baldwin, 1955b). It then seemed very attractive to consider this entire volume as the dwelling region of cosmic rays; such a model was capable of explaining all the observations available then, including the high degree of 1sotropy of the radiation and the few $\mathrm{g} \cdot \mathrm{cm}^{-2}$ of matter traversed. However, it can easily be shown that the latter observation needs a cosmic ray residence time of about $10^{8}$ years in the halo 
model. Furthermore detailed study on the background galactic radio emission suggests that the radio spurs, which were once thought to be large scale features extending right into the halo region could only be local phenomena (Baldwin, 1967). If this were so then the genuine galactic radio emitting region will also be considerably flattened and approach closer to the size of the radio disk. Furthermore, recent observations on cosmic rays strongly suggest that the residence time of cosmic rays is likely to be less than $10^{7}$ years (Brown et al., 1973a; Webber et al., 1973a; O'Dell et a1., 1973; Daniel and Stephens, 1967). These observations would therefore indicate that even in the galactic model the effective cosmic ray storage region is the galactic disk. Possible intermediate regions between the galactic disk and galactic halo have also been referred to in the past (Daniel and Stephens, 1970). Thus in summary the current status in the field seems to be in favour of the galactic disk model though one could still argue about the effective extend of the thickness of the Disk for cosmic ray containment. 1.3 Scope of the Present Review:

It is evident that if one is to investigate and deduce useful information from a model in which cosmic rays are generated, propagated and stored in our galaxy, one would have to make the best use of all available information on the Galaxy, its dimensions and its constituents. In particular, one would need as much data as possible on the interstellar medium and its physical state. We have, therefore, judiciously collected from the relevant literature all such information of direct concern to us here and summarized them in Chapter 2. Since in this model, the confinement region is adequately circumscribed, one is able to work out a theoretical formulation of the 
propagation of cosmic rays in the Galaxy; this is described in Chapter 3. The present theoretical treatment is however limited to a situation in which the cosmic rays are in a state of equilibrium and are distributed homogeneously in the residence volume. While such a simplified treatment has been forced upon us because of the otherwise intrinsic complexities and difficulties encountered, the assumptions regarding the equilibrium nature of the cosmic rays and its homogeneous distribution in the Galaxy seem to be well founded on the basis of our existing knowledge. In the fourth Chapter, we examine and interpret the observational data on cosmic rays in relation to their propagation in the Galaxy.

The fact that cosmic rays traverse a region of cosmic space pervaded by a tenuous gas, weak magnetic flelds and radiation fields, implies that cosmic rays which are literally immersed in them, should interact with them in a wide variety of ways. Many of these lead to the production of radiations ranging the entire electromagnetic spectrum - from radio waves to gamma rays; these are described in Chapter 5. Fina11y, recent studies in astrophysics have unmistakably highlighted the possible roles that cosmic rays are likely to play in some aspects of galactic dynamics such as the hydrostatic equilibrium of interstellar gas, gravitational instability and formation of clouds, heating of interstellar gas etc. These are briefly covered in Chapter 6 . Though we still have a long way to go towards a fuller understanding of the propagation of cosmic rays in the Galaxy and its implications, we can already see that much progress has been achieved particularly during the last decade or so.

Before proceeding further, we would like to emphasize the propagation of cosmic rays in the Galaxy is a subject in which theoretical 
formulations including model building on the one hand, and experimental observations on the other, have to advance in parallel, continuously checking the findings of one with that of the other, and incoporating suitable modifications in the model and the theory to build-up an increasingly self consistent picture. Real progress is often made in being able to rule out or discredit models whose predictions are in contradiction with observations rather than in being able to propose new models which are consistent with existing observations. Furthermore, in this subject, wherein one finds room for highly speculative Ideas, there is immense scope for widely differing viewpoints. It is therefore too much to expect in one review article extensive coverage to be accorded to all view points so far proposed; a certain amount of selectivity on the part of the authors seems inevitable to make the presentation coherent. The reader's indulgence and understanding are therefore solicited for any apparent biases and omissions that might have resulted in an attempt to achieve this. Finally, as supplementary reading we will like to refer the reader to a number of other review articles, and references therein, on topics of allied and overlapping interest (Parker, 1969; Meyer, 1969; Daniel and Stephens, 1970; Shapiro and Silberberg, 1970; Simpson, 1971; Wentze1, 1974). 


\section{GENERAL DESCRIPTION OF THE GALAXY}

The Galaxy is an assembly of about $10^{11} \mathrm{stars}$ together with much gas and dust. Its spheroidal central region is enveloped by a flat disk of stars in which the spiral arms are embedded. The Galaxy is in a state of rotation around this/ is a slowly rotating near-spherical halo containing a low density of high velocity stars and globular clusters. The Sun is situated close to the equatorial plane of the Galaxy and about two thirds of the distance from the centre to the edge of the Disk. In this Chapter, we shall summarize the general properties of the Galaxy relevant to our present purpose as inferred from observations to-date.

\subsection{Dimensions and Mass Density}

The Galaxy has no sharp physical boundaries, and its dimensions are not known with high precision. The distance $R_{0}$ between the Sun and the Centre is taken to be $10 \mathrm{kpc}$ (Arp, 1965; Schmidt, 1965), though this value could be as small as $8.5 \mathrm{kpc}$ (Toomre, 1972). However, in this Chapter all parameters which depend upon $R_{0}$ are given on the basis of $R_{0}=10 \mathrm{kpc}$. From the neutral hydrogen distribution, one can deduce the diameter of the Galaxy covering the major features (but excluding the extended Outer Arm), as about $30 \mathrm{kpc}$. The thickness of the Disk varies from region to region, the optfcal disk being flatter than the radio disk. A study of the distribution of the stellar population in a direction perpendicular to the galactic plane shows that the effective thickness defined as the distance between half density points is different for different classes of stars; for Population I Cepheids it is about $180 \mathrm{pc}$ (Walraven et al, 1958) while for F8-G8 dwarfs it is about $300 \mathrm{pc}$ (Elvius, 1965). Also the thickness 
increases considerably to more than about $1 \mathrm{kpc}$ towards the Centre (Oort, 1938). The non-thermal radio emission in the Galaxy is symetric about the Centre within a radius of $8.7 \mathrm{kpc}$ having a thickness of $750 \pm 100 \mathrm{pc}$ (Baldwin, 1967). This would mean that the Sun is outside this symmetric disk of emission. Beyond the $8.7 \mathrm{kpc}$ region there is an outer disk of uniform emission which is relatively thinner with a probable thickness of about 500 pc.

According to Schmidt (1965) the density distribution in the Galaxy can be well approximated by a spheroid having an axial ratio 1:20 with the following features: (i) equidensity surfaces in the form of similar spherolds, (ii) a mass point at the Centre and (iii) a shell outside. The mass distribution could then be represented as:

$$
\begin{array}{ll}
\rho=3.930 \mathrm{R}^{-1}-0.02489 \mathrm{R} & \text { for } \mathrm{R}<9.71 \mathrm{kpc} \\
\rho=1449.2 \mathrm{R}^{-4} & \text { for } R>9.71 \mathrm{kpc}
\end{array}
$$

where $\rho$ is expressed in solar mass $M_{0}$ per $\mathrm{pc}^{3}$ and $R$ is the distance from the Centre in $\mathrm{kpc}$; the central mass point has a value of $7 \times 10^{9} \mathrm{M}$, that of the spheroid is $8.2 \times 10^{10} \mathrm{M}_{\odot}$ and of the shell $9.3 \times 10^{10} \mathrm{M}_{\odot}$ From this the total mass of the Galaxy is $1.8 \times 10^{11} \mathrm{M}_{\odot}$, half of which is within a spheroidal surface drawn through the Sun; the total mass in the form of gas is only about 5 to $7 \times 10^{9} \mathrm{M}_{0^{*}}$ Observationa1ly, the hypothetical central mass point consists of about $10^{9} \mathrm{M}_{\mathcal{O}}$ within a radius of $100 \mathrm{pc}, 10^{8} \mathrm{M}$ within $10^{7} \mathrm{M}$ within $1 \mathrm{pc}$ (Oort, 1973). The density near the sun is 0.145 $\mathrm{M}_{0} \mathrm{pc}^{-3}$ i.e., $10^{-23} \mathrm{~g} \mathrm{~cm}^{-3}$. Further one may also add that the escape velocity near the Sun is nearly $380 \mathrm{~km} \mathrm{~s}^{-1}$; the age of the Galaxy is about 1.2 $\mathrm{x} 10^{10} \mathrm{yrs}$; and the period of rotation in the neighborhood of the Sun is $2.5 \times 10^{8}$ y rs. 


\subsection{Structure}

Much of the information on the structure of the Galaxy comes from the study of the $21 \mathrm{~cm}$ emission profiles of the Galaxy. In Figure 2.1 is shown the neutral hydrogen distribution in the galactic plane. This figure is derived from Kerr and Westerhout (1965) and Kerr (1967), but the scale of the radius has to be enlarged by a factor of $10 / 8.2$ to agree with the new value of $R_{0}=\mathrm{kpc}$. In this figure the region marked 1 in the centre is the nuclear disk of radius about $900 \mathrm{pc}$ with an inner and outer section, the inner one being a rapidly rotating disk and the outer one, probably, with some radial motion as well. The region marked 2 constitutes the "3-kpc Arm" at about $4 \mathrm{kpc}$ from the centre; it has an outward motion together with rolling. The total neutral hydrogen mass in the Nucleus is estimated to be about $3 \times 10^{6} \mathrm{M}_{0}$ while in the $3 \mathrm{kpc}$ Arm it is $3 \times 10^{7} \mathrm{M}_{\odot}$ (Rougoor and 0ort, 1960). At about $5 \mathrm{kpc}$ from the centre is the "ScutumNorma Arm" (region marked 3 in Figure 2.1); the "Sagittarius Arm" (region No. 4 is at a distance of $7.5 \mathrm{kpc}$. The Sun is situated in the inner edge of the "Orion Arm" or the "Carina Cygnus Arm" (region No. 5) which is about $10 \mathrm{kpc}$ from the Centre. At about $3 \mathrm{kpc}$ beyond the Sun, we have the prominent "Perseus Arm" (region No. 6). Outside the Perseus Arm one can see the "Intermediate Arm" (region No. 7) beyond which is located the faint "Outer Arm", (region No. 8). The spiral feature of the Galaxy can in general be represented by a logarithmic multi-arm spiral with a pitch angle of about $85^{\circ}$ (Pskovskil, 1965). It is difficult to decide from Figure 2.1 whether a single spiral arm continues over very large distances in the Galaxy or whether the observations can be interpreted as a collection of short spiral arms. The first alternative will suggest that the 

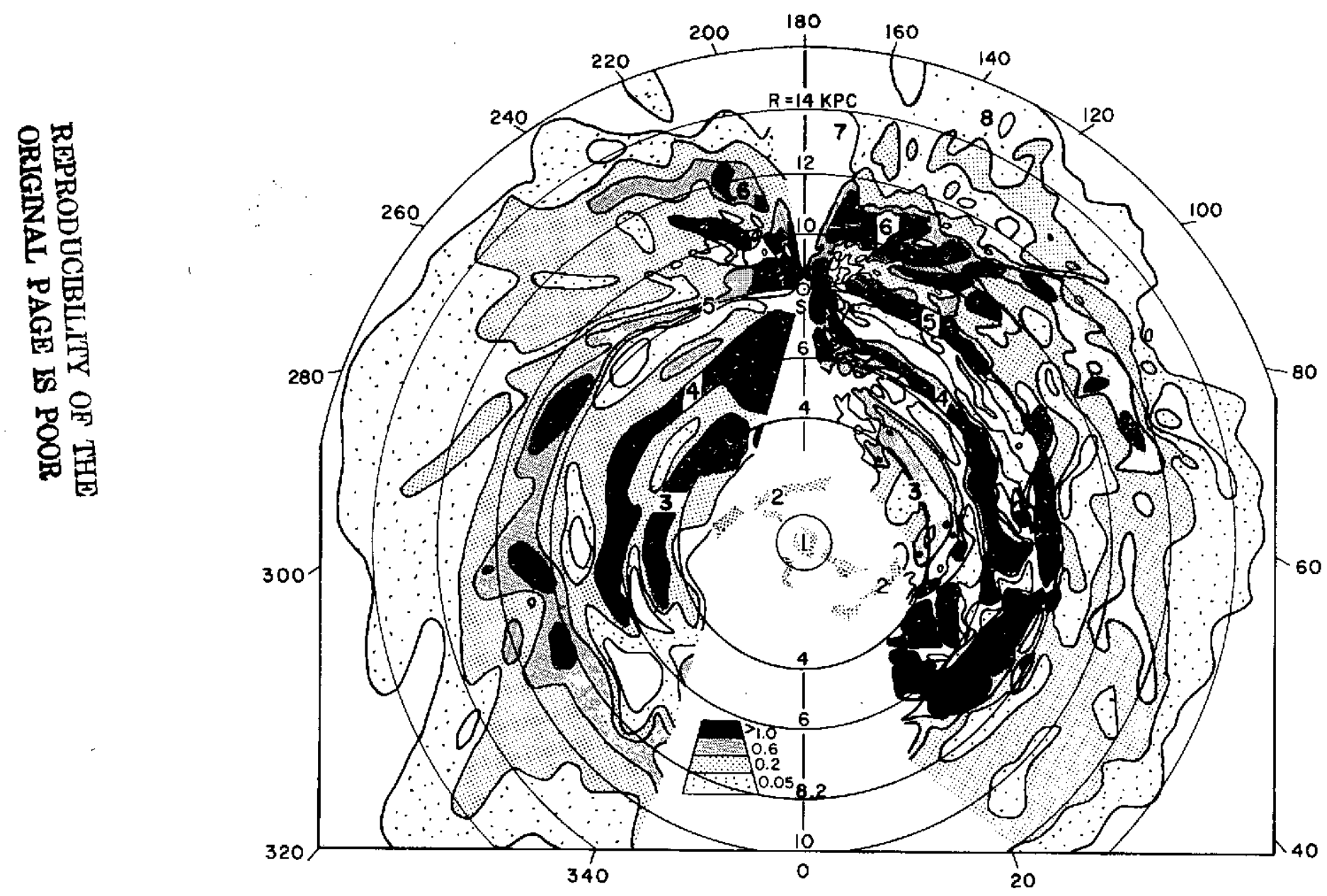

Fig. 2.1: Distribution of neutral hydrogen in the Galaxy according to Kerr and Westerhout (1965) in which the "3 kpc Arm" and the nucleus have been inserted from Kerr (1967). The scale of radius corresponds to Ro $=8.2 \mathrm{kpc}$. The inset near the bottom indicates the value of $\mathrm{n}_{\mathrm{H}}$ in atoms $\mathrm{cm}^{-3}$, which does not apply to the central region. 
spiral arms exist over many rotation periods of the Galaxy while if the 1atter were the case, 1t would indicate that they form and dissolve even In a time duration as short as the galactic rotation period.

Many spur features have been noticed from studies of the galactic non-thermal radiation extending from the equator to high latitudes. of the three which have been well recognized, the "North Polar Spur" is the most intense; the other two are the Cetus Arc and the Loop III. The radio brightness of these features falls off with increasing latitude; at the same time not much is known about their brightness at low latitudes, where they merge into the intense radiation from the Disk. Furthermore, although at low latitudes they appear to lie roughly perpendicular to the galactic equator, there is no evidence to reveal whether they cross the galactic plane. There are many ways of understanding the origin of these spurs: (a) old supernova remnants of large size relatively close to the Sun (Hanbury Brown et al., 1960); (b) cosmic ray pressure in the Disk can, according to Parker (1965), lead to instabilities in the galactic magnetic fields whereby loops of field may be blown out to large values of $z$ to make them appear like long fingers roughly perpendicular to the galactic plane; (c) effect of helical tubes of magnetic field wrapped round the local spiral srin (Rougoor, 1966); and (d) large scale features in the galactic Halo. Nevertheless, there are as yet no decisive arguments to establish any one of the above possibilities nor to rule out others (Baldwin, 1967).

Apart from the spurs, which are mostly high latitude features of radio continuum in the Galaxy, one can also identify many structures from the neutral hydrogen distribution in the Disk. The most prominent of these seen from Figure 2.1 are: (1) the extension of the Orion Arm towards 
the Anti Centre; (i1) the outer strong feature in the longitude range $200^{\circ}$ to $240^{\circ}$ as the extension of the Persus Arm; and (iii) the connecting links between the Nucleus and the $3 \mathrm{kpc}$ Arm. Many inter arm links, though not very prominent, can also be recognized providing support for the assumption that for many purposes, e.g. the cosmic ray propagation, the Galaxy is a simple uniform disk with many large and small scale structural features. It is also pertinent to point out that the hydrogen layer beyond $\mathrm{R}=9 \mathrm{kpc}$ shows a systematic distortion with a positive deviation in the region of galactic longitudes $20^{\circ}<1<140^{\circ}$ and a negative deviation at $200^{\circ}<1<340^{\circ}$; the deviation is nearly maximum in the direction perpendicular to the SunCentre direction (Kerr and Westerhout, 1965). It is suggestive that this distortion is a general feature of the outer region of the Galaxy.

The association with the Galaxy of globular clusters well beyond $\mathrm{z} \sim 2.5 \mathrm{kpc}$ makes one surmise that the Galaxy extends also in the $\mathrm{z}$-direction beyond the apparent boundary of the Disk mentioned in Section 2.1. Moreover, there is evidence for the existence of neutral hydrogen at distances of $1 \mathrm{kpc}$ above the Perseus Arm with an estimated density of nearly $3 \times 10^{-2}$ hydrogen atoms $\mathrm{cm}^{-3}$, decreasing to about $10^{-2}$ hydrogen atoms $\mathrm{cm}^{-3}$ at 1.5 kpc (Kepner, 1970). Also, from a study of the negative velocity clouds at high latitudes, one infers that there should exist considerable amount of gas outside the Disk so as to offer an efficient mechanism to retard the infalling of the intergalactic gas (Oort, 1969). All these indicate the existence of a physical halo around the Disk of the Galaxy in which most of the gas is 11kely to be highly ionized. This deduction is further strengthened by the fact that it is difficult to imagine a sharp boundary 
between the optical disk and the near-intergalactic space which differs markedly in the physical conditions, namely matter density and magnetic field (Ginzburg, 1967). The shape of the Halo may be nonhomogeneous spheroid, with no sharp boundary but perhaps extending up to about $10 \mathrm{kpc}$ from the plane of the Disk. The non-thermal radiation at high latitudes also suggests the existence of a radio halo with a radius probably of about $18 \mathrm{kpc}$ (Baldwin, 1967). However, the absolute brightness from the Halo is in question; this aspect will be further examined in Section 5.3. Notwithstanding all these, a detailed study of 18 spiral galaxies shows no evidence of any associated large radio halo postulated for our own galaxy (Mi11s, 1967).

\subsection{Interste1lar Gas}

The existence of interstellar gas was first suggested by Hartmann (1904) following his observation of stationary absorption lines of ionized calcium in a spectroscopic binary. Today, the basic information on interstellar matter is derived from the intensity of neutral hydrogen emission line at $21 \mathrm{~cm}$. If as is generally believed atomic hydrogen is the major constituent of interstellar gas, one can reliably assign values for the density of gas in different regions of the Galaxy from the $21 \mathrm{~cm}$ observations. Following such a consideration it is seen that the distribution of the mean density in the galactic plane has a broad maximum of about 0.6 hydrogen atoms $\mathrm{cm}^{-3}$ between $R=5$ and $12 \mathrm{kpc}$ falling off both inwards and outwards (Kerr and Westerhout, 1965). Nevertheless the density of neutral hydrogen in the central region increases from about 0.2 atoms $\mathrm{cm}^{-3}$ at $R=600 \mathrm{pc}$ to about 5 atoms $\mathrm{cm}^{-3}$ at $\mathrm{R}=100 \mathrm{pc}(0 \mathrm{ort}, 1973)$. The density of gas may further increase towards the inner parts, where the observed density of ionized hydrogen itself is about 15 atoms $\mathrm{cm}^{-3}$ (Lequeux, 1967). The ionized 
hydrogen constitutes only about $3 \%$ of the total gas in interstellar space. The mean density of gas in the Halo, which is fully ionized is about (5 - 10) $x 10^{-3}$ atoms $\mathrm{cm}^{-3}$. The thickness of the layer of hydrogen gas obtained from a series of tangential measurements gives a value of about $280 \mathrm{pc}$ between half density points. However, around $4 \mathrm{kpc}$ from the centre the thickness is only about $150 \mathrm{pc}$, which drops to a value of about $100 \mathrm{pc}$ close to the Centre. This demonstrates that the total mass in the form of gas in the central region of the Galaxy is negliglble and that the central mass density resides essentially in the stars. At large radit, the thickness of the gas layer increases considerably; it may be as high as several hundreds of parsecs beyond $15 \mathrm{kpc}$. The distribution of gas perpendicular to the galactic plane is symmetric and is approximately of Gaussian shape, except at large distances where it is wider. The mean thickness of 280 pc between half density points corresponds in the z-component to a dispersion of $\sigma_{z}=120 \mathrm{pc}$. Mention may also be made here that the distribution of stars in the z-plane of the Galaxy in the neighborhood of the Sun is also approximately a Gaussian; and the Sun is situated at about 10 pe north of the equatorial plane of symmetry (Elvius, 1965).

Although the large scale physical properties of interstellar gas are known fairly well, the detailed structure could be very complex. The interstellar medium is generally known to be composed of cool, dense clouds embedded in a hot, rarefied intercloud medium. The clouds vary widely in size from about $0.1 \mathrm{pc}$ to $100 \mathrm{pc}$ (Van de Hulst, 1958); their density and distribution in the Galaxy is not uniform. Combining the observational data as summarized by Van Woerden (1967), and the theoretical study on the pressure equilibrium between the clouds and the intercloud medium (Field 
et a1., 1969), one can describe the physical properties of an average cloud as follows: the diameter of the cloud is about $10 \mathrm{pc}$; the density of neutral hydrogen $\mathrm{n}_{\mathrm{H}} \approx(20-30)$ atoms $\mathrm{cm}^{-3}$; the free electron density $\mathrm{n}=22 \times 10^{-2} \mathrm{el} \cdot \mathrm{cm}^{-3}$ (Stephens, 1971); and the temperature is about $100^{\circ} \mathrm{K}$. The fraction of volume occupied by these clouds in the equatorial plane of the Galaxy is about $(2-4) \%$. The distribution of clouds in the $z$ plane of the Galaxy is similar to that of neutral hydrogen. The total mass in the form of clouds is about $70 \%$ of the interstellar gas. The intercloud medium which is considered to fill the Galaxy uniformly, has a density in the equatorial plane of $\mathfrak{n}_{\mathrm{H}} \approx 0.2$ atoms $\mathrm{cm}^{-3}$ at a temperature of about $(6-10)$ $\mathrm{x} 10^{30} \mathrm{~K}$ with a free electron density $\mathrm{n} \mathrm{e}^{\approx} 4 \times 10^{-2} \mathrm{el} \cdot \mathrm{cm}^{-3}$, as inferred from the dispersion measures of pulsars (Prentice and Ter Haar, 1968). The distribution of this hot gas in the $z$ plane is slightly wider than that of cold gas with a dispersion $\sigma_{z}=160 \mathrm{pc}$. As can be seen from the free electron densities in the clouds and intercloud medium, the fraction of ionized gas is about $3 \times 10^{-2}$.

Except for a few elements, the chemical composition of interstellar matter has not been determined directly from observations. The estimate made on the strength of interstellar absorption lines, though somewhat less certain, reveal that the composition of $g$ as is about the same as in Population I stars (Spitzer, 1968a). The relative abundances of various elements as summarized by Greenberg (1968) and Allen (1965) are given in Table 2.1.

Recent observations by the Copernicus satellite indicate (Morton et al., 1973; Jenkins et al., 1973) that the abundances of elements like C, O, S1 and Fe 
Table 2.1

Relative Abundances of Some of the Elements in Interstellar Medium

\begin{tabular}{|c|c|c|c|c|c|}
\hline Element & $\begin{array}{c}\text { Relative } \\
\text { number } \\
\text { of atoms }\end{array}$ & $\begin{array}{c}\text { Relative } \\
\text { mass }\end{array}$ & Element & $\begin{array}{r}\text { Relative } \\
\text { number } \\
\text { of atoms }\end{array}$ & $\begin{array}{c}\text { Relative } \\
\text { mass }\end{array}$ \\
\hline $\mathrm{H}$ & 1.0 & 1.0 & Si & $2.5 \times 10^{-5}$ & $7.0 \times 10^{-4}$ \\
\hline $\mathrm{He}$ & $1.2 \times 10^{-1}$ & $4.8 \times 10^{-1}$ & $\mathrm{P}$ & $3.2 \times 10^{-7}$ & $9.8 \times 10^{-6}$ \\
\hline Li & $1.0 \times 10^{-9}$ & $7.0 \times 10^{-9}$ & $\mathrm{~S}$ & $2.8 \times 10^{-5}$ & $9.0 \times 10^{-4}$ \\
\hline $\mathrm{Be}$ & $2.5 \times 10^{-10}$ & $2.3 \times 10^{-9}$ & $\mathrm{Cl}$ & $1.0 \times 10^{-6}$ & $3.5 \times 10^{-5}$ \\
\hline B & $6.3 \times 10^{-10}$ & $6.8 \times 10^{-9}$ & Ar & $7.6 \times 10^{-6}$ & $3.0 \times 10^{-4}$ \\
\hline $\mathrm{C}$ & $2.5 \times 10^{-4}$ & $3.0 \times 10^{-3}$ & $\mathrm{~K}$ & $7.9 \times 10^{-8}$ & $3.1 \times 10^{-6}$ \\
\hline $\mathrm{N}$ & $1.3 \times 10^{-4}$ & $1.7 \times 10^{-3}$ & $\mathrm{Ca}$ & $1.6 \times 10^{-6}$ & $6.4 \times 10^{-5}$ \\
\hline 0 & $7.9 \times 10^{-4}$ & $1.3 \times 10^{-2}$ & $\mathrm{Ti}$ & $5.0 \times 10^{-8}$ & $2.4 \times 10^{-6}$ \\
\hline $\mathbf{F}$ & $2.5 \times 10^{-7}$ & $4.8 \times 10^{-6}$ & $\mathrm{Cr}$ & $8.9 \times 10^{-8}$ & $4.7 \times 10^{-6}$ \\
\hline $\mathrm{Ne}$ & $6.3 \times 10^{-4}$ & $1.2 \times 10^{-2}$ & $\mathrm{Mu}$ & $6.3 \times 10^{-8}$ & $3.5 \times 10^{-6}$ \\
\hline $\mathrm{Na}$ & $1.3 \times 10^{-6}$ & $2.9 \times 10^{-5}$ & $\mathrm{Fe}$ & $2.5 \times 10^{-5}$ & $1.4 \times 10^{-3}$ \\
\hline $\mathrm{Mg}$ & $4.5 \times 10^{-5}$ & $1.1 \times 10^{-3}$ & Co & $5.4 \times 10^{-8}$ & $3.2 \times 10^{-6}$ \\
\hline $\mathrm{A} 1$ & $1.8 \times 10^{-6}$ & $4.8 \times 10^{-5}$ & $\mathrm{Ni}$ & $1.1 \times 10^{-6}$ & $6.6 \times 10^{-5}$ \\
\hline
\end{tabular}


vary from region to region, and that some of these trace elements are under abundant compared to the universal abundances. This can be due to the locking up of these elements in dust grains (Spitzer, 1968b); such depletion of trace elements in clouds resulting from accretion on grains seems to gain support from the fact that the temperature of the clouds are higher than that predicted using normal abundances (Goldsmith et al., 1969). Since energetic cosmic ray particles while traversing matter in space do not distinguish very much between individual atoms and small grains, one can, for purposes of cosmic ray propagation, consider the cosmic abundances in Table 2.1 as representative of the interstellar $g$ as. Apart from individual atoms, the interstellar gas also contains molecules. The abundance of molecular hydrogen is relatively low in common clouds and high in dense dark clouds; however, from observations one can only say that the total abundance in the Disk hardly exceeds that of atomic hydrogen (Pikelner, 1967). During more recent years, molecules like OH, CH, $\mathrm{CN}$ and even more complex ones are being observed in interstellar space. As pointed out earlier, a small fraction of the gas also exists in the form of dust grains which are presumably formed in the clouds. It has been suggested that the interstellar extinction can be well understood $1 f$ grains consist of graphite cores with ice mantles, while the intrinsic polarization can be understood as due to small graphite flakes (Greenberg, 1967).

\subsection{Magnetic flelds}

The first observation on stellar polarization (Hiltner, 1949; Ha11, 1949) almost certainly required for its understanding the existence of 
interstellar magnetic fields. At present there exist many different methods of determinlng the strength and orientation of the magnetic field in the Galaxy. They are: (a) Stellar polarization; (b) Faraday rotation; (c) Zeeman splitting; (d) radio polarization; and (e) radio brightness distribution. We shall now very briefly summarize the results from these studies.

\section{(a) Stellar polarization}

It is generally accepted that stellar polarization is produced by the scattering of light by grains, which get aligned by the galactic magnetic field. Early measurements by Hiltner (1949) and Hall (1949) showed that the magnetic field is predominantly parallel to the galactic plane and that the major component lies along the spiral arm. A helical field in the spiral arm has also been postulated (Hoyle and Ireland, 1961). From an extensive analysis of recent data Mathewson, (1968) has proposed a model In which the local field is wound in a spiral of pitch angle $7^{\circ}$ around the surface of an elliptical cylinder, whose axis is in the galactic plane and at right angle to the direction of the centre; the spiral is sheared through $40^{\circ}$ anti-clock wise as seen from the north galactic pole.

\section{(b) Faraday rotation}

A plane polarized radio wave of waveleng th $\lambda$, while traversing an Ionized medium with a frozen-in magnetic fleld, undergoes a rotation of the plane of polarization. The rotation measure $R=\psi / \lambda^{2}$, where the angle of rotation $\psi$, is in radians and $\lambda$ is in meters, is numerically expressed as $R=8.1 \times 10^{2} \int_{0}^{L} B_{11} n_{e} d s$, where $B_{11}$ is the longitudinal component of the magnetic fleld in Gauss and $d s$ is the element along the line of sight in pc. When the rotation measure obtained for extragalactic sources is plotted against the galactic latitude of the respective sources (Berge and Scielstad, 
1967), It is seen that most of the rotation takes place in the Galaxy. From these observations, taking $\left\langle n_{e} \cdot B_{11} \cdot L\right\rangle \approx 2 \times 10^{-5}$ towards the galactic pole, and substituting $\mathrm{n}_{\mathrm{e}}=3 \times 10^{-2} \mathrm{e} 1 . \mathrm{cm}^{-3}$ as the mean electron density for $L=300 \mathrm{p}$ in the Disk across the galactic plane, one finds

$B_{11} \approx 2 \mu \mathrm{G}$ as the mean longitudinal component of the local magnetic field In the Galaxy (Shakeshaft, 1968). Rotation measures from pulsars also Indicate that $B_{11}$ is about $3 \mu G$ (Smith, 1968). These values 1mply that the strength of the galactic magnetic fleld would be $\succsim 3 \mu \mathrm{G}$. In the light of rotation measures obtained from extragalactic sources, a revised analysis of stellar polarization suggests (Mathews on and Nicholls, 1968), that the hellcal component of the magnetic field, needed to account for the stellar polarization, is superimposed on the large scale longttudinal fleld in the Galaxy, which is along the spiral arm.

\section{(c) Zeeman Splitting}

In the case of Zeeman splitting of excited lines, the separation between the two components polarized circularly in opposite directions is given as $\Delta v=2.8 \times 10^{5} \mathrm{~B}_{1 \mathrm{l}}$, where $\mathrm{B}_{1 \mathrm{l}}$ is expressed in Gauss and $\nu$ in $\mathrm{Hz}$. Recent observations of Zeeman splitting of neutral atomic hydrogen line at $21 \mathrm{~cm}$, both in absorption against strong radio sources and in emission, towards different galactic directions, show a wide variation in the magnitude of $B_{11}$ (Verschuur, 1969). It has been pointed out by Verschuur that these observations can reveal information on1y about the fields in high density, low temperature $\mathrm{H}-1$ clouds and not about interstellar fields in general; it is also suggested that the cloud field can be effectively detached from the more general field in interstellar space. Thus one finds that a detailed study of the Zeeman splitting would enable one to elicit information about magnetic fields in dense clouds existing in interstellar space. 


\section{(d) Radio Polarization}

Since the non-thermal component of the radio continuum is due to synchrotron radiation enitted by relativistic electrons, one would expect that polarization measurements would shed light on the orientation of large scale magnetic fields in the Galaxy responsible for the emission. However, the Faraday rotation within ionized clouds tends to depolarize the radiation and hence one is unable to derive information on the magnetic field without a knowledge of the exact distribution of ionized hydrogen along the line of sight. Observations in the local region of space, where the line of sight crosses not more than a few clouds of ionized hydrogen, suggest that the field is well aligned to the local arm (Lequeux, 1969).

\section{(e) Radio Brightness Distribution}

Information on the strength of large scale magnetic fields in the Galaxy comes from the unique relationship between the cosmic ray electron spectrum in interstellar space and the spectral distribution of the radio continuum along any given direction in the Galaxy. This method was first introduced by Biermann and Davies (1960) after which many such investigations have been made in recent years. The mean perpendicular component of the magnetic field in the Galaxy averaged over the line of sight in different directions in the galactic plane is $6 \mu \mathrm{G}$ (Section 5.3). This value combined with that derived in (b) above on the longitudinal component leads to a fleld strength of (6-8) $\mu \mathrm{G}$ for the large scale magnetic fields. The magnetic field in the Halo is probably about (2-3) $\mu$ G.

\subsection{Radiation Fields}

Radiation fields existing in the Galaxy extend right from the radio to the gammaray domain. Part of this radiation is the result of diverse 
processes occurring inside the Galaxy; the rest has 1 ts origin in the Universe at large. We shall briefly summarize here our knowledge of the radiation energy density in the Galaxy in the different spectral regions. (a) Radio Region

The electromagnetic radiation in this regime is essentially due to synchrotron radiation emitted by cosmic ray electrons in the Galaxy. At frequenciee below a few $\mathrm{MH}$, the free-free absorption in interstellar space prevents this radiation from propagating through the Galaxy, while for the entire range in excess of this frequency, galactic space is transparent. If the differential spectral shape of the radio brightness in the sky is expressed as $I \alpha v^{-\alpha}$, it is then found that $\alpha \approx 0.0$ at about $2 \mathrm{MHz}$ slowly Increasing to about 0.8 at frequencies above $2 \times 10^{2} \mathrm{MHz}$; below $2 \mathrm{MHz}$, the spectrum has a positive slope due to interstellar absorption. The total energy density $\rho_{\mathrm{ph}}$ in this field is about $10^{-6} \mathrm{eV} \mathrm{cm}^{-3}$ in the neighborhood of the Sun.

\section{(b) Microwave and Far Infra-red Regions}

This is the most important region of the radiation field existing in space. Considerable enhancement of the sky brightness in the microwave region far above that expected from a simple extrapolation of the galactic radio continuum was first discovered by Penzias and Wilson (1965). This has been interpreted by Dicke et al. (1965) as due to the existence of the Untversal Black Body Radiation, the inescapable remnant of the "big-bang" origin of the Untverse (Gamow 1948). If this were so, then this radiation would permeate the whole Universe; within the accuracy of measurements so far made on the isotropy of this radiation the universal nature of this radiation has been substantiated. The observed intensity in the frequency 
region of $10^{3}-10^{5} \mathrm{MHz}$, is consistent with the Rayleigh-Jeans part of the black body radiation curve at $T=2.68^{\circ} \mathrm{K}$ (for details see Peebles, 1971). If the black body nature of this radiation is correct, then the corresponding energy denstty would be $4 \sigma \mathrm{T}^{4} / \mathrm{c} \approx 4.73 \times 10^{-3} \mathrm{~T}^{4}=0.25 \mathrm{eV}$. $\mathrm{cm}^{-3}$, where $\sigma$ is Stefan's constant; the mean energy of the photon $\langle\varepsilon\rangle=$ $2.7 \mathrm{kT}=6.3 \times 10^{-4} \mathrm{eV}$, where $\grave{\mathrm{k}}$ is Boltzmann's constant.

(c) Infra-red Region.

Observations in the Infra-red region are so far made using ground based instruments; they are thus exposed to the thermal radiation-environment of the atmosphere. Because of this reason, determination of the extraterrestrial continuum radiation becomes difficult and one is constrained to observations relating to sources only (Webbink and Jeffers, 1969). On the other hand, theoretical estimates of the infra-red radiation from interstellar grains have also been made In the past; recently Krishnaswamy (1970) has calculated the expected spectral distribution in the solar neighborhood from different grain models and from this it is found that the energy density in the region (3-15) $\times 10^{7} \mathrm{MHz}$ is $\approx 10^{-3} \mathrm{eV} \mathrm{cm}^{-3}$. The energy density decreases sharply at higher frequencles.

(d) Visible Region

The radiation field in this small band of electromagnetic spectrum comes from stellar emission. The interstellar radiation field calculated by suming up essentially the contributions from stars of various spectral types (Z1mmermann, 1964) is consistent with a grey body radiation at $\mathrm{T} \approx 10^{4} \mathrm{~K}$ with a dilution factor of $10^{14}$. The total energy density in this frequency band is about $0.5 \mathrm{eV} \mathrm{cm} \mathrm{cm}^{-3}$, which includes a little of near 
infra-red and near ultraviolet radiations; the mean photon energy is about $2 \mathrm{eV}$.

\section{(e) Ultra Violet Region}

Our knowledge of the radiation fleld in this domain is again very poor, and measurements are yet to be made in the future. However, recent calculations of the radiation density in the frequency interval (1.2-3.2) $\times 10^{9} \mathrm{MHz}$ indicate that $\rho_{\mathrm{ph}} \approx 4 \times 10^{-2} \mathrm{eV} \mathrm{cm}^{-3}$ (Habing, 1968).

(f) $X$ and Gamma-ray Regions

Reliable measurements are now available on the diffuse background of $\mathrm{X}$-rays and gamma rays. These observations suggest that the radiation field above $1 \mathrm{keV}\left(2.4 \times 10^{11} \mathrm{MHz}\right)$ is of extragalactic origin. The total energy density in this domain $1 \mathrm{~s}$ only about $4 \times 10^{-5} \mathrm{eV} \mathrm{cm}^{-3}$; also it can be inferred from the spectral shape that the radiation density decreases with increasing energy.

From the above summary, 1t becomes clear that the important radiation fields in the Galaxy are the UnIversal Black Body Radiation and the stellar optical emission. 


\section{Cosmic Ray Propagation in the Galaxy-Theoretical Aspects}

In what has been sumarlzed in Chapter 1 , we have identified the most probable region of confinement of cosmlc rays to be the Galaxy; we then surveyed, in Chapter 2, those descriptions and features of the Galaxy of relevance to our considerations here. We now proceed to examine theories applicable to the problem of propagation of cosmic rays in galactic space. In attempting to formulate a rigorous theory for this, one encounters diverse difficulties because of the many inherent uncertainties and complexttles of the problem. Some of these difficulties can be lessened significantly if cosmic rays are assumed to be in a state of equilibrium, and are distributed homogeneously within the confinement volume. Indeed, It is found that there does exist a reasonable body of evidence in favor of such an assumption though recently some workers have pointed out observations which seem contrary to it. These aspects are first sumarized in this Chapter. We then proceed with a brief description of models of diffusion of cosmic rays in interstellar space; their relative merits are also critically examined. Following this, a general equation describing the transformation in the chemical composition and energy spectrum of cosmic rays during their propagation is discussed, Finally, several theoretical models of propagation of nucleonic and electronic components of cosmic rays, often employed to understand the experimental results, are described. We will like to state here that in the theoretical formulation of the propagation problem, we w111 not separately deal with propagation in the "source region" since the physical characteristics of this region, even if it turns out in the future to be important in the overall problem of cosmic ray propagation, is poorly understood at present. 
3.1 Quasi-stationary State and Spatial Homogenity of Cosmic Rays

In order to study the propagation of cosmlc rays in the region of space where they reside, it is essential first to enquire whether cosmic rays extend throughout this region in a quasi-stationary state or with notable fluctuations. Although the first alternative seems to be the conservative line of approach, it is widely adopted in all interpretative studies since it is amenable to theoretical formulations and constructive advancement compared to the latter in which there are considerable difficulties and very many unknowns. We shall, therefore, first examine the evidence from cosmic ray prehistory to see whether one can fustify the simple hypothesis that cosmic rays are in a quasi-stationary state in the Galaxy as a whole. Most of our information on cosmic ray prehistory is obtained from the analyses of stable and unstable cosmogenic nuclei found in meteorites (Lal, 1966). Such studies, Involving nuclel of half lives ranging from days to millions of years, reveal that the average cosmic ray $f 1 u x$, integrated over the mean lives of the respective radio lsotopes, has remained unchanged (Gelss, 1963). Scheaffer et al. (1963) have shown that the mean intensity over the last $500 \mathrm{yrs}$. has been the same as that averaged over $5 \times 10^{5}$ yrs within $\pm 10 \%$. Furthermore, the gross cosmic ray intensity has also remained unchanged within a factor two over extended periods in the past up to $10^{9}$ yrs (Voshage and Hintenberger, 1963; Anders, 1965; Lipschutz et a1. 1965). A similar analysis using moon rocks (Finkel et al. 1971) suggests that the time averaged flux of cosmic ray protons above a $\mathrm{GeV}$ has been the same during the past 5 million yrs as the contemporary one. Hence, one feels fustified in taking the viewpoint that the cosmic ray intensity has not changed, over a few rotations of the Galaxy, by more than a factor of two. 
It needs to be pointed out here that above $10^{3} \mathrm{yrs}$ the lack of detailed information does not permit one to rule out possible large scale fluctuations to occur with periods which lie between the half-lives of the radio isotopes used. For instance, large scale fluctuations during the present epoch with characteristic periods of a few times $10^{4}$ yrs will not contradict the observational deduction that the present cosmic ray intensity is nearly the same as that averaged over the last $10^{6}$ or $10^{9}$ yrs (Parker, 1968). However, the failure to detect any large scale variation so far would appear to favor cosmic rays to be in a state of quasi-equilibrium over long periods of time.

As for spatial homogenity, a detailed examination of non-thermal radio continuum towards various galactic directions by Anand et al. (1968a) reveals that the cosmic ray intensity in the Galaxy when averaged over the line of sight is the same in all regions within a factor of two. However, it is difficult to rule out, at this stage, possible gradients to exist either in localized regions of space, such as active sources and source regtons, the spiral arms, or in the Halo perpendicular to the galactic plane. In fact, it has recently been suggested, on the basis of radio astronomical and cosmic gamma ray observations, that contemporary cosmic rays are not uniformly distributed in the Galaxy (Bignami and Fichte1, 1974; Stecker et a1. 1974). Nonetheless, the present status of these postulations are either tentative or speculative; also their quantitative effect on the theoretical treatment of the propagation problem is not clear. Finally, there are as yet no major and reliable attempts made to formulate theoretically the propagation problem in a situation wherein cosmic rays are not in a state of equilibrium in the Galaxy; it is, however, almost certain that it is likely to attract greater interest and attention of the researchers in the years to come. 
In consequence of the above observations, we will confine ourselves, in this review, to the extensive work so far undertaken under the basic assumption that cosmic rays are in a state of quasi-equilibrium in the Galaxy.

\subsection{Models of Diffusion of Cosmic Rays}

The motion of a cosmic ray particle in the Galaxy is influenced by the nature of the magnetic fields therein. In this section, we shall briefly describe some of the important models for the diffusion of cosmic rays in interstellar space.

\subsubsection{Motion of charged particles in a magnetic field and their diffusion}

In 1ts simplest form the motion of a charged particle in a magnetic field having large scale irregularities, is associated. with a drift velocity across the magnetic field and is given as

$$
\mathrm{v}_{\mathrm{d}} \approx \mathrm{r}_{\mathrm{B}} \cdot \mathrm{v} / 1
$$

where, 1 is the characteristic scale of field inhomogenity, $r_{B}=E \sin \theta / 300 z B$ is the radius of curvature in $\mathrm{cm}$ of a relativistic particle of charge $\mathrm{Z}, \mathrm{E}$ in $\mathrm{eV}$ is the energy of the particle moving at an angle $\theta$ to the magnetic field of strength $B$ expressed in Gauss, and $v$ is the velocity of the particle. Two points need to be noted here. One, for a typical situation in interstellar space,Equation 3.1. would require that for the escape from the Galaxy of particles of energy, say $1 \mathrm{GeV}$, the scale length has to be as small as $10^{15} \mathrm{~cm}$. Two, this expression, to be applicable, requires that the motion of the particle is adiabatic all along the line of force and $r_{B} \ll 1$, a condition unlikely to be true in interstellar situation. 
The above difficulties can be circumvented by proposing a variation in the model wherein cosmic rays have to traverse scattering centers, such as magnetic clouds and shock waves, and that the random walk of particles resulting from multiple scattering in the medium leads to diffusion (Ginzburg and Syrovatskil, 1964; Parker, 1963). In case of magnetic clouds with size $1_{c}$ very much in excess of the radius of curvature of the particle $r_{B c}$ inside the cloud (i.e. $r_{B c} \ll 1_{c}$ ), one can write for $\lambda_{s}$ the scattering mean free path

$$
\lambda_{\mathrm{s}} \approx 1.0 / \mathrm{n} \cdot \mathrm{1}_{\mathrm{c}}^{2}
$$

where $\mathrm{n}$ is the number density of clouds in space. It can then be seen that for typical interstellar conditions $\lambda_{s}$ is independent of energy at least up to $10^{16} \mathrm{eV}$. Nevertheless, at much higher energies (i.e. $\mathrm{r}_{\mathrm{BC}}>1_{\mathrm{c}}$ ), $\lambda_{s}$ depends strongly on the energy and can be written as

$$
\lambda_{s} \approx \frac{1.0}{\mathrm{n}_{\mathrm{c}}{ }_{\mathrm{C}}^{2}}\left(\mathrm{r}_{\mathrm{Bc}} / \mathrm{I}_{\mathrm{c}}\right)^{2}
$$

Since the diffusion coefficient $D \approx(1 / 3) \lambda_{\mathrm{s}} \mathrm{v}$, it is seen that when $\mathrm{n}_{\mathrm{c}}{ } \mathrm{s} \mathrm{x}$ $10^{-60} \mathrm{~cm}^{-3}$ and $1_{c} \sim 20 \mathrm{pc}$, the value of $\mathrm{D}$ is $\sim 5 \times 10^{29} \mathrm{~cm}^{2}$. It can easily be shown that such a large value of diffusion coefficient would allow cosmic rays to escape from the Disk within a short period of $\sim 10^{4}$ yrs unless one invokes strong reflection at the boundary for an effective confinement in the Disk; on the other hand, it would be quite consistent with a model wherein cosmic rays are confined to the Halo. It is important to point out that in these considerations one has neglected the existence of any ambient magnetic field in the intercloud region in which the particles are not likely to satisfy the relation $r_{B} \ll 1$ required of the treatment; rather, one has made use of the idea of single particle scattering. 
Plentiful realization has now emerged that, since the energy density of cosmic rays is comparable to the magnetic field energy "and the kinetic energy of the gas, the collective effect of the relativistic cosmic ray gas, which is essentially a collisionless hot plasma, assumes significant proportions. We shall now briefly examine the collective effects of cosmic rays on their diffusion.

\section{2 .2 Self scattering of cosmic rays}

Earlier hopes that scattering of cosmic rays by small scale irregularities in the general galactic magnetic field will be adequate for our understanding of cosmic ray propagation and confinement in the Galaxy have not been well borne out, and it has become imperative to look for other possible scattering mechanisms as we11. In this search a process which seems to offer considerable scope for the future is the collective effects of cosmic rays during their propagation in the Galaxy. For a recent survey on this subject we refer the reader to an interesting article by Wentzel (1974).

In interstellar space, the relativistic cosmic ray plasma, streams along the general large scale magnetic field lines; tn this process they collectively excite a spectrum of hydromagnetic waves. These are Alfven waves which propagate in the ionized component of the interstellar gas along the streaming direction of the particles. Under the condition when the cosmic ray performs one gyration around the general magnetic fleld B while traversing one Alfven wavelength $\lambda$, there is resonant interaction between the particles and these waves. The condition for this resonant self scattering (or cyclotron resonant scattering) can be written as $\lambda=r_{B} v_{11} / c$ 
where $v_{11}$ is the velocity of the particle along $B$. Also, the scattering mean free path $\lambda_{s}$ can be written as

$$
\lambda_{\mathrm{S}} \approx \frac{2}{\pi} \mathrm{r}_{\mathrm{B}} \quad \frac{\text { energy in the ambient magnetic field }}{\text { energy in the magnetic waves }} \quad 3.4
$$

These relations highlight the following very interesting features: (a) cosmic rays of different energies resonate with different waves; (b) since at each energy range cosmic rays make their own waves, off which they scatter, this self scattering process becomes increasingly ineffective at higher energies because of the falling power law nature of the primary spectrum; and (c) at a typical energy of about $1 \mathrm{GeV}$ where the flux of particles, and hence the wave growth, is large, the scattering mean free path is exceedingly small and is only $\sim 10^{-2}$ pc. In fact, it is the last feature which makes this process more attractive from the point of view of the diffusion of cosmic rays in the Galaxy.

When there is a systematic general streaming or drift of cosmic rays relative to the background interstellar plasma and if the drift velocity becomes comparable to the Alfven speed, the waves excited by cosmic rays scatter them and thereby inhibit the streaming (Wentze1, 1969; Kulsrud and Pearce, 1969). The damping of the Alfven waves in the Disk is due to Eriction between the ionized hydrogen set in motion by the waves and the neutral hydrogen. From these ideas Wentzel (1974) has shown that the mean bulk velocity $\langle v\rangle$ is related to the Alven speed $v_{A}=B /\left(4 \pi n_{i}\right)^{2}$ and the number of cosmic rays $n(>p)$ above a momentum $p$ as follows:

$$
\langle\mathrm{v}\rangle=\frac{2+\beta}{3} \mathrm{v}_{\mathrm{A}}+\frac{0.19}{\beta-1}\left(\frac{\mathrm{T}}{10^{3}}\right)^{0.4} \mathrm{~N}_{1}^{\frac{1}{2}} \mathrm{~N}_{\mathrm{H}} / \mathrm{N}(>\mathrm{p}) \mathrm{cm} \mathrm{s}^{-1}
$$

Here $\mathrm{T}$ is the temperature of the medium and $\mathrm{n}_{i}$ and $\mathrm{n}_{H}$ are the ionized and neutral densitites in the medium, and $\beta$ is the differential spectral index 
of the cosmic ray spectrum. In the case of fully ionized medium, the damping of hydromagnetic waves is due to wave-wave interactions and as a result, the energy dependence of the bulk velocity (second term of Equation 3.5) becomes $\alpha \mathrm{E}^{0.75}$. If one uses typlcal values for the parameters applicable to interstellar space, one finds that particles of about $1 \mathrm{GeV}$ are well scattered, those at about $10 \mathrm{GeV}$ are weakly scattered and those above 100 $\mathrm{GeV}$ are hardly scattered at all.

Thus, in spite of the many attractive features of the mechanism of self-scattering, it is disappointing that in its present form it is unable to explain the high degree of the observed isotropy of cosmic rays of energy $10^{1 \mathrm{ll}}-10^{12} \mathrm{eV}$.

\subsubsection{Instability model}

In this model, wherein pioneering work has been carried out by Parker (1969), it is assumed that the cosmic ray gas diffuses freely along the general galactic field lines, which are parallel to the plane of the Galaxy, except in regions of small scale magnetic irregularities. In fact it has been shown recently (Earl, 1974) that if coherent propagation of cosmic rays is effective in interstellar space, they can be transported almost scatter free along the field lines. Diffusion perpendicular to the plane occurs elther due to the ergodic nature of the field lines or the stochastic property of the field. The cosmic ray gas escapes from the Disk by inflating the outer reglons of the field.

The presence of the suprathermal cosmic ray gas in the large scale magnetic field of the Galaxy does not affect the usual hydrodynamic waves even though the thermal plasma and the suprathermal gas are tied to the 
magnetic lines of force; the physical reason for this is that the suprathermal gas is so mobile, except when self-scattering becomes important, that it can redistribute itself with ease along the lines of force, thereby avoiding compression and rarefaction associated with the hydromagnetic modes. Though the suprathermal wave propagation has also some effect on the thermal gas, the role of the thermal gas is to provide enough inertia to make the field act as a rigid guide along which the suprathermal mode propagates. Thus, we find the intimate connection between the gas-field system and the propagation of cosmic rays. We shall not qualitatively examine the manner in which diffusion of cosmic rays occurs across the galactic plane.

Firstly, on account of the ergodic nature of the galactic field along which the suprathermal gas streams, it gradually tends towards isotropy and uniform density distribution. The characteristic time in which the cosmic ray gas relaxes towards this uniform distribution is of the order of the dimension of the region of space divided by a few times the Alfven speed in the ionized component of the gas. The thermal gas, on the other hand, permits the fleld to expand and decrease its energy while streaming along the lines of force. The cosmic ray gas would then inflate the outer portions of the field where the cosmic ray pressure becomes larger than the magnetic pressure $\left(P>B^{2} / 8 \pi\right)$ and therefore tends to escape.

Secondly, the stochastic nature of the field which may prove even more effective, is a direct consequence of the random turbulence of the gas in which the field is embedded (Jokipii and Parker, 1968; 1969a). It has been shown (Jokipii and Parker, 1969b) that any given line of force random walks from the equatorial plane of the Galaxy to its surface, while traversing 
distances of $(5-10) \times 10^{2} \mathrm{pc}$. Thus the cosmic rays are transported across the Disk by the very stochastic nature of the magnetic lines of force. If this is true, then the cosmic rays do not have to travel far to enter regions in which $\mathrm{B}^{2} / 8 \pi<\mathrm{P}$; inflation would then set in regions at about every $10^{3}$ pc along each tube of magnetic flux. The instability resulting from the inflation is opposed by the gas perturbations and by the tension in the magnetic field, while the cosmic ray pressure increases the inflation. Such a situation may lead to a state of turbulence, and cosmic rays may escape out of the Galaxy efther freely or probably carrying with them part of the magnetic lines of force.

\subsection{The diffusion process and 1sotropy of cosmic rays}

It has been seen in Section 3.2 that in the various models described so far, cosmic rays stream along and across the interstellar magnetic field lines and diffuse in all directions. However, if the streaming of particles is not the same in all directions, it would result in an anisotropy. Detection of such an anisotropy, in a region with dimensions of the order of the particle's radius of curvature, would give information on the net streaming velocity of cosmic rays in any particular direction and thus lead to an insight into their diffusion and propagation. Thus far, observations have yielded only upper-1imits to the magnitude of the anisotropy. We shall now try to understand the implications of these observational results on the basis of the diffusion of cosmic rays in interstellar space.

The cosmic ray anisotropy is defined as $\delta=\left(I_{\max }-I_{\min }\right) /\left(I_{\max }+\left(I_{\min }\right)\right.$, where $I$ is the intensity in a given direction. From a study of the sidereal variation of underground muon intensity, it has been shown by Elliot 
et al. (1970) that at energies $\gtrsim 10^{2} \mathrm{GeV}$, the observed anisotropy is $\preccurlyeq 2 \mathrm{x}$ $10^{-4}$. It has been pointed out by Parker (1969) that at these energies the irregularities in the interplanetary magnetic field itself could significantly isotropise cosmic rays because the gyroradius of a proton at $10^{2} \mathrm{GeV}$ is only $0.4 \mathrm{AU}$ in a typical magnetic field strength of $5 \times 10^{-5}$ gauss. Indeed, it has been shown by trajectory calculations (Speller et al. 1972) that at rigidities less than a few hundred GV, the particle's arrival directions are we11 scattered in the solar system. Therefore, taking into account the response of their detector as a function of primary energy, Speller et al. (1972) have concluded that in the energy region $10^{2}-10^{3}$ $\mathrm{GeV}, \delta<1.5 \times 10^{-4}$. Since the anisotropy can be related to the streaming velocity of cosmic rays as $\mathrm{v} \approx \mathrm{c} \delta /(2+\beta)$, one finds that for the observed value of $\delta$, the corresponding streaming velocity is $\Omega_{10} \mathrm{kms}^{-1}$. Such a low anisotropy would require for particle escape from the Disk a lifetime of $\gtrsim 3 \times 10^{7} \mathrm{yrs}$ in conflict with the estimates of $\sim 10^{6}$ yrs deduced for galactic disk models (Parker, 1969).

The low streaming velocity of cosmic rays deduced from the low anisotropy observed by Elliot et al. (1970) have stimulated many workers to seek plausible explanations. It was first proposed by Jones (1970a) that the discrepancy in the cosmic ray life time for escape referred above could be due to fluctuations resulting from the statistical nature of the cosmic ray source distribution, which at the present happens by chance to give an extremely low anisotropy at the Earth. Soon after, Ramaty et al. (1970a) proposed the statistical discrete-source model of local cosmic rays in which 
they started with the acceptance that the general streaming velocity is indeed as low as that suggested by the low observed anisotropy. These authors further assumed that cosmic ray sources are confined to the Disk and are supernova explosions, each treated as a random point source in space and time; they then characterized cosmic ray propagation in interstellar space by three dimensional isotropic diffusion with a constant mean free path $\lambda$. From such an approach these authors noted that in order to account for the observed anisotropy, one needs $\lambda<10^{-1} \mathrm{pc}$; furthermore to accommodate the observed ratio of the flux of light to medium nuclei at relativistic energies, one needs $10^{-2} \mathrm{pc} \preccurlyeq \lambda \leqslant 10^{-1} \mathrm{pc}$. Also, in this model the life time for escape from the Disk is $\gtrsim 10^{7} \mathrm{yrs}$ and hence the life times of various nuclei are determined by their nuclear interactions and not by escape. These authors further pointed out that the discrepancy between isotropy and the otherwise needed escape life time of $\sim 10^{6} \mathrm{yrs}$, cannot be accounted solely by fluctuations as suggested by Jones (1970a) unless the propagation is strictly one dimensional. Following this, Jones (1971) presented an argument in favor of an essentially one dimensional propagation of cosmic rays and showed that the distribution of streaming velocity is strongly peaked towards zero; thus the probability of seeing low observed antsotropy is more likely than any other value. Nevertheless, in this model one has to accept that the observed low anisotropy is only local to us and is due to an accident of our position in space and time.

A serious difficulty in the statistical discrete-source model of Ramaty et al. (1970a) is the small scale size of $\lesssim 0.1$ pc for the magnetic irregularities in interstellar space. Additionally, a proper understanding 
of the observed abundance of positrons, deuterons and secondary 1ight nuclel was also posing problems. In order to overcome these difficulties, Lingenfelter et al. (1971) modified their earlier model by considering the combined effect of one dimensional diffusion of cosmic rays along the magnetic fleld lines and to the three dimensional random walk of these field lines, a treatment first discussed by Getmantsev (1963). Thus, the resultant motion of a particle is due to a compound diffusion with two mean free paths $\lambda_{p}$ and $\lambda_{m}$ to describe the scattering of particles along the field lines, and the random walk of the fleld lines, respectively. The quantitative treatment of the compound diffusion model of Lingenfelter et al. (1971) expressed in a simplified fashion by Allan (1972) will now be briefly described. As a result of the random walk of the field lines, the total length $L_{m}$ of the line of force along which the cosmic rays propagate from the source to the Earth can be written as $L_{m}=d^{2} / \lambda_{m}$ where $d$ is the distance between the source and the Earth. Since particles have to traverse this distance $L_{m}$ by a process of one-dimensional diffusion, we can, by a similar argument, write for the total particle path length $\mathrm{L}_{\mathrm{p}}=\mathrm{L}_{\mathrm{m}}{ }^{2} / \lambda_{\mathrm{p}}=$ $d^{4} /\left(\lambda_{m}^{2} \lambda_{p}\right)$. In consequence, the transmit time is proportional to $d^{4}$ in this case instead of $\mathrm{d}^{2}$ for simple diffusion. Furthermore, the increase in transit time, compared with straight line motion, is by a factor $d^{3} / \lambda_{m}{ }^{2} \lambda_{p}$; hence the outward streaming is reduced correspondingly and the mean anisotropy can be written now as

$$
\delta_{\mathrm{av}}=\lambda_{\mathrm{m}}{ }^{2} \lambda_{\mathrm{p}} / \mathrm{d}^{3}
$$

Setting $\lambda_{m} \sim \lambda_{p} \sim \lambda$, and employing a value of $L_{p} \sim 10^{6}$ pc corresponding to a few $\mathrm{g} \mathrm{cm}^{-2}$ of matter traversed by cosmic rays in interstellar space having a mean density of $\sim 1$ atom $\mathrm{cm}^{-3}$, one deduces a value of $\lambda \sim 20$ pc for $\delta \sim 3 \mathrm{x}$ $10^{-4}$. 
It is further argued by Allan (1972) that in the compound diffusion model each magnetic tube of force is required to have a minimum width much in excess of the distance coursed by the solar system with a velocity of $220 \mathrm{~km} \mathrm{~s}^{-1}$ with respect to the galactic frame over the period of a few years when the measurements were in progress. Consequently, the observational data corresponds to the microscopic anisotropy within a single tube of force $\delta_{s}$ appropriate to the simple one dimensional diffusion, which is written as

$$
\delta_{\mathrm{s}} \approx \lambda_{\mathrm{p}} / \lambda_{\mathrm{m}}=\lambda_{\mathrm{p}} \lambda_{\mathrm{m}} / \mathrm{d}^{2}
$$

If this were so, then $\mathrm{d} \sim 1 \mathrm{pc}$ and $\lambda \sim 10^{-2} \mathrm{pc}$ leading us back to the same difficulty of too short a mean free path.

Thus in summary, if one accepts serlously the low value of anisotropy experimentaliy determined so far by only one group (Elliot et al. 1970), we are yet to propose an acceptable and internally consistent physical process which will explain it in a natural sequence. Therefore, 1t goes without saying that such a. consequential observation should be measured by other experimenters with greater accuracy and reliability in the years. to come. And finally, one is left wondering that perhaps in all these attempts a fundamental concept in the propagation and confinement of cosmic rays in the Galaxy is still missing. 


\subsection{Genera1 Formulation of the Propagation of Cosmic Rays in Interstellar}

\section{Space}

So far we endeavoured to understand the mode of propagation of cosmic rays in interstellar space in relation to their dynamical properties. The obvious impression one gets from the extsting theories is that there is perhaps no unique manner in which the diffusion of cosmic rays in the Galaxy can be treated quantitatively. Furthermore, during propagation in interstellar space, the composition and energy spectrum of cosmic rays are modified due to nuclear interactions and other energy loss processes; incorporating these in the diffusion theory is likely to introduce further difficulties. Nonetheless, it is essential to formulate quantitatively the propagation of cosmic rays in a simple form in order to make a beginning to understand and interpret observations. Modifications and improvements could then be incorporated, or new formulations attempted, depending on the degree of success of the theoretical predictions to explain observations. Towards this we shall first proceed to develop a general formulation for the propagation of cosmic rays, and then discuss various simplifying approximations which lead to different models of propagation; in this formulation the dynamical aspects of cosmic ray diffusion will not be taken into account.

The general equation of propagation, which allows for spatial diffusion, energy losses, acceleration and transformation of chemical composition in interstellar space can be written in the form (Ginzburg and Syrovatskii, 1964).

$$
\frac{\partial N_{i}}{\partial t}+\underline{\nabla} \cdot \underline{J}_{i}+\frac{\partial}{\partial E}\left(b_{i} N_{i}\right)+p_{i} N_{i}=Q_{i}(E, \underline{r}, t)
$$


where $\mathrm{N}_{i}$ is the density of particles of the $i^{\text {th }}$ kind defined as

$$
\mathrm{N}_{1}(\mathrm{E}, \underline{\underline{r}}, \mathrm{t})=\frac{1}{\mathrm{v}} \int \mathrm{I}_{\mathbf{1}}(\mathrm{E}, \underline{\mathrm{r}}, \mathrm{t}, \underline{\mathrm{k}}) \mathrm{d} \underline{\Omega}_{k}
$$

Here, $I_{i}(E, \underline{r}, t, \underline{k})$ is the intensity in the direction $\underline{k}$, and $\underline{d}_{\Omega_{k}}$ is the element of solid angle in k-space; if the intensity is isotropic, then $N=4 \pi I / v$. We shall now examine Equation 3.8 in detail. The first term on the left hand side of Equation 3.8: This term represents the rate of change of density of cosmic rays per unit time due to processes described by the other terms in the equation. The second term on the left hand side of Equation 3.8: This term is the rate of net flow of particles in the co-ordinate space, where $J_{1}(E, \underline{r}, t)$ is the net flow of particles defined by the mode of diffusion; it can be written in general as

$$
\underline{J}=-\underline{D} \nabla N+\underline{v}^{\prime} N
$$

where $\underline{D}$ is the diffusion tensor and $\underline{v}^{\prime}$ is the velocity of the scattering centers. Under isotropic diffusion approximation,

$$
\underline{J}=-D \nabla N
$$

where $D$ is the diffusion coefficient.

The third term on the left hand side of Equation 3.8: This includes the continuous energy loss by particles due to processes, such as ionization and synchrotron and inverse-Compton radiation and continuous energy gain from possible acceleration mechanisms as in the case of Fermi process. The net change in the energy per unit time can be written as

$$
\frac{d E}{d t}=b_{i}(E)
$$

One can also include in princlple an additional term $-\frac{1}{2} \partial^{2} / \partial E^{2}\left(e_{i} N_{i}\right)$ which can take care of fluctuations in the continuous variation in energy. 
In this case the term $e_{i}=e_{i}(E)=d / d t\left\langle\Delta E^{2}\right\rangle$ is the mean square of the energy increment per unit time. However, we shall not introduce such a term in our considerations since, firstly, the amount of fluctuation expected in the energy loss by cosmic rays during their propagation in the Galaxy is expected to be small, and secondly, it is believed that the statistical acceleration mechanism does not play any important role in the propagation.

The fourth term on the left hand side of Equation 3.8: This term corresponds to catastrophic energy losses, such as the emission of high energy bremsstrahlung quanta by an electron and spallation of heavy nuclei resulting in the loss of particle identity; here $p_{i}$ is the probability per unit time for such losses.

The term on the right hand side of Equation 3.8: This represents the production rate of cosmic rays and can be written as

$$
Q_{i}(E, \underline{r}, t)=q_{i}(E, \underline{r}, t)+\sum_{k} \int P_{1 k}\left(E^{\prime}, E\right) N_{k}\left(E^{\prime}, \underline{r}, t\right) d E^{\prime}
$$

Here, the first term on the right represents the production rate from sources situated at a distance $\underline{r}$ at time $t$, while the second describes the secondary particle production as a result of catastrophic collisions. $P_{i k}\left(E^{\prime}, E\right)$ is the probability of production per unit time of the $i^{\text {th }}$ type of particle with energy $E$ from catastrophic collisions of particles of the $k^{\text {th }}$ type having energy $E^{\prime}$.

The general solution of Equation 3.8 can be obtained by making use of Green's function which satisfies the following equation (Syrovatskil, 1959),

$$
\frac{\partial G}{\partial t}-D \nabla G+\frac{\partial}{\partial E}(b G)+p G=\delta\left(\underline{r}-\underline{r}^{\prime}\right) \delta\left(E-E^{\prime}\right) \delta\left(t-t^{\prime}\right)
$$


The solution of Equation 3.8 can then be written as

$$
N_{i}(E, \underline{r}, t)=\iiint \underline{d r}^{\prime} \int d E^{\prime} \int d t^{\prime} Q_{1}\left(E^{\prime}, \underline{r}, t\right) G\left(E, \underline{r}, t ; E^{\prime}, \underline{r}^{\prime}, t^{\prime}\right)
$$

Here, the Green's function $G$ is called the propagation function since Equation 3.14 represents the propagation part of the general diffusion equation. For infinite space, the propagation function has the form

$$
G\left(E, \underline{r}, t ; E^{\prime}, \underline{r}^{\prime}, t^{\prime}\right)=\frac{1}{b(E)(4 \pi \lambda)^{3 / 2}} \exp \left[-\left(\underline{r}-\underline{r}^{\prime}\right)^{2} / 4 \lambda-\tau^{\prime} / T\right] \delta\left(t-t^{\prime}, \tau^{\prime}\right) \quad 3.16
$$

where $\tau$ ' is the time taken for the particle of energy $E$ ' at $\underline{r}^{\prime}$ to reach ' $\underline{r}$ with an energy $E$ due to continuous energy loss processes and can be written as

$$
\tau^{\prime}\left(E, E^{\prime}\right)=t-t^{\prime}=E^{\prime} \frac{d E}{b(E)}
$$

A1so

$$
\lambda=\int^{E} \frac{D(E)}{b(E)} d E
$$

which becomes $D \tau^{\prime}$, when the diffusion is independent of particle energy; $T=1 / p$ is the mean life time for catastrophic loss processes, where $p$ is the probability of such processes per unit time.

For quasi-steady state condition, where the explicit time derivatives In Equation 3.8 vanish, we get

$$
\begin{aligned}
& \mathrm{N}_{i}(\mathrm{E}, \underline{\mathbf{r}})=\iiint \underline{\mathrm{d}}^{\prime} \int \frac{\mathrm{d} \mathrm{E}^{\prime} Q_{i}\left(\mathrm{E}^{\prime}, \underline{r}^{\prime}\right)}{|\mathrm{b}(\mathrm{E})|(4 \pi \lambda)^{3 / 2}} \exp \left[-\left(\underline{\mathbf{r}}-\underline{\underline{r}}^{\prime}\right)^{2} / 4 \lambda-\tau^{\prime} / \mathrm{T}\right] \\
& =\iiint d r^{\prime} \int \frac{d E^{\prime} Q_{i}\left(E^{\prime}, r^{\prime}\right)}{|b(E)|\left(4 \pi D \tau^{\prime}\right)^{3 / 2}} \exp \left[-\left(r-r^{\prime}\right)^{2} / 4 D \tau^{\prime}-\tau^{\prime} / T\right]
\end{aligned}
$$

In the special case of an unbounded confinement space with a homogeneous distribution of sources therein, the above solution reduces to 


$$
N_{1}(E)=\frac{1}{b(E)} \delta_{E^{\prime}}, \exp \left[-\frac{1}{T} \delta_{E^{\prime}}^{E}, \frac{d E^{\prime \prime}}{b\left(E^{\prime \prime}\right)}\right] Q_{1}\left(E^{\prime}\right) d E^{\prime}
$$

The limits of the integral over $E^{\prime}$ is determined from the behavior of the energy loss parameter $b(E)$ with energy. If $T$ depends on the energy of the particle, Equation 3.20 can be written, without loss of generality, in the form

$$
N_{i}(E)=\frac{1}{\mid b(E)} \delta_{E^{\prime}} \exp \left[-\int_{E^{\prime}}^{E} d^{\prime \prime} /\left\{b\left(E^{\prime \prime}\right) T\left(E^{\prime \prime}\right)\right\}\right] Q_{i}\left(E^{\prime}\right) d E^{\prime} \quad 3.21
$$

This equation reveals that the density of particles does not depend upon the diffusion mechanism but only on the continuous and catastrophic energy loss processes. Such a situation is also applicable in space with finite boundary conditions wherein the diffusion of particles is very slow.

The exact solution of Equation 3.8 in space with finite boundary and an arbitrary distribution of sources, is quite difficult to obtain even under steady state conditions. However, one can evaluate Equation 3.19 numerically for any arbitrary source distribution by incoporating spatial dependence of the energy 1oss processes through Equation 3.17. In case of homogeneous distribution of sources both in space and time, it is customary to assume that the diffusion is very fast within the finite volume and that particles are lost by slow leakage from the boundary. In such a situation, the diffusion term in Equation 3.8 is replaced by a catastrophic loss term with an equivalent leakage Iife time $\tau$ which is the residence time of cosmic rays in the finite volume of confinement; $\tau$ can elther be energy dependent or independent. Under this situation, Equation 3.21 would take the form

$$
N_{1}(E)=\frac{1}{\mid(b(E)} \mid f_{E^{\prime}} Q_{i}\left(E^{\prime}\right) \exp \left[-\int_{E^{\prime}}^{E} \frac{d E^{\prime \prime}}{b\left(E^{\prime \prime}\right)}\left\{\frac{1}{\left(T^{\prime} E^{\prime \prime}\right)}+\frac{1}{\tau\left(E^{\prime \prime}\right)}\right\} \quad 3.22\right.
$$

This solution is widely used by researches in the field and it is called the steady state solution for cosmic ray propagation. 
We considered so far the general formulation of the diffusion problem in the Galaxy. The next step is to apply it to a situation involving the various observations within the framework of models so far proposed. These are reviewed in what follows in Section 3.5 .

\subsection{Quas1-Equilibrium Models}

Almost all interpretative studies on the chemical composition and energy spectrun of cosmic rays are based on propagation models under quasi-equilibrium conditions. For the sake of clarity in presentation, we shall separately consider the electron component because of the dissimilar nature of dominant energy loss processes associated with their propagation in space.

\subsubsection{Nucleonic Components}

In case of nucleonic components, at all energies except perhaps $\gtrsim 10^{18} \mathrm{eV}$, the only important continuous energy loss process is ionization; this loss becomes increasingly severe at sub-relativistic energies, where one may also have to take into account elastic collisfons particularly with nuclei of low mass. The catastrophic losses are primarily due to inelastic collisions and leakage from the confinement space. In inelastic collisions, heavier nuclei suffer fragmentation and lose their identity, giving rise to nuclei of lower charges; the associated change in the velocity of the fragment is inappreciable except at sub-relativistic energies. The fragmentation parameters involved are, in general, energy dependent at low energies and seem to saturate at the relativistic domain. For protons, inelastic collisions at energles above the threshold for meson production is of relevance though its contribution in the general propagation can be disregarded except in spectal circumstances where there is slow diffusion in a confinement region with high matter density, such as source regions. 
Two major approaches have been adopted so far to study the propagation of cosmic ray nuclei in space. In the first, only interaction losses are taken into account by assuming that all particles arrive at a given point in space after the traversal of a fixed distance. An example of this is the "Slab Model" in which it is assumed that particles of a given energy traverse a fixed length of trajectory and hence the same amount of matter. In the second, leakage is also folded in by considering the confinement of cosmic rays in a given region of space; the "Steady State Model" in which all possible vacuum trajectories are allowed with a finite probability belongs to this category.

\section{(a) The Slab Model}

The simplified diffusion equation which includes both ionization loss and fragmentation can be written as (Aizu et al. 1960; Ray, 1960; Fichtel and Reames, 1966).

$$
\frac{d}{d x}\left[w_{1}(E) j_{i}(E, x)\right]=-\frac{j_{1}(E, x)}{\lambda_{i}(E)} w_{i}(E)+\sum_{k>i} \frac{j_{k}\left(E^{\prime}, x\right)}{\lambda_{k}\left(E^{\prime}\right)} P_{i k}\left(E^{\prime}\right) w_{i}(E) \quad 3.23
$$

Here, $j$ defines the differential intensity of particles, $E$ the energy per nucleon, $w(E)=d E / d x$ is the rate of energy loss per nucleon per unit path length, $x$ the path length in $\mathrm{g} \mathrm{cm}^{-2}, \Lambda_{1}$ the attenuation mean free path in $\mathrm{g} \mathrm{cm}^{-2}$ and $P_{i k}$ the fragmentation parameter defined earlier. $\Lambda_{i}$ can be defined as $\lambda_{i} /\left(1-p_{i i}\right)$ where $\lambda_{i}$ is the interaction mean free path and $P_{i 1}$ the fragmentation parameter relating to the probability of the particle retaining its identity after interaction; this relation also holds when $i$ represents a group of nuclei but does not apply for protons in which case $P_{i i}$ has to 
be replaced by the inelasticity parameter. The solution of Equation 3.23 under such an approximation would be

$$
\begin{aligned}
& j_{i}(E, x)=\frac{\left|w\left(E^{\prime}\right)\right|}{|w(E)|} j_{1}\left(E^{\prime}, 0\right) \exp \left[-\int_{0}^{x} \frac{d x^{\prime}}{n_{1}\left(E^{\prime}, x^{\prime}\right)}+\int_{0}^{x} \frac{\left|w_{i}\left(E^{\prime}, y\right)\right|}{\left|w_{1}(E)\right|} x\right. \\
& \sum_{k>i} \frac{j_{k}\left(E^{\prime \prime}, y\right)}{\lambda_{k}\left(E^{\prime \prime}\right)} P_{i k} \quad\left(E^{\prime \prime}\right) d y \exp \left[-\int_{y}^{x} \frac{d z}{\Lambda_{i}^{\left(E^{\prime}, z\right)}}\right]
\end{aligned}
$$

The first term here represents the single particle propagation, while the second corresponds to the propagation of the $i^{\text {th }}$ type of nuclei produced during the traversal of nuclel heavier than the $i^{\text {th }}$ type, or simply, the secondary particle propagation; $j_{i}\left(E^{\prime}, 0\right)$ is the intensity of particles at the source with energy $E^{\prime}$ such that ionization loss reduces it to $E$ at the point of observation. Since $A$ is a function of energy, its energy dependence is taken care of at each depth. In the second term, $E^{\prime}$ corresponds to the energy of the $1^{\text {th }}$ type of nuclei during production at $y$ due to the corresponding $k^{\text {th }}$ type of nuclet with the relevant energy $E^{\prime \prime}$. The integration is carried out numerically from the heaviest particle onwards so that at each depth the flux of the $k^{\text {th }}$ type would be available for the propagation of the lighter nuclel $\mathbf{i}<\mathrm{k}$.

Even under the slab approxtmation, certain variations in the method of computation have been introduced leading to very different results. Two such variations may be mentioned here. (i) A distribution in the path length about the mean amount of matter traversed $\langle x\rangle$ by the particles, has been constdered by Balasubrahmanyan et al. (1965). In this case the flux of particle can be written as

$$
f_{1}(E)=\int_{0}^{\infty} f_{i}(E, x) p(x) d x
$$


where $f_{1}(E, x)$ is defined by Equation 3.23 and $p(x) d x$ is the normalized distribution such that $\int_{0}^{\infty} p(x) d x=1$. From a consideration of a cosmic ray source situated at a distance $\underline{x}$ from the observer, in a homogeneous medium with isotropic particle diffusion, it is found that $p(x) d x$ is approximately Gaussian and the distribution of path length is of the form

$$
p(x) d x=\frac{(5 / \pi)^{\frac{1}{2}}}{1.8858\langle x\rangle} \exp \left[-\frac{5}{4}\left(\frac{x-\langle x\rangle}{\langle x\rangle}\right)^{2}\right] \quad 3.26
$$

Th1s model necessarily predicts a spatial gradient of the Intensity of cosmic rays by virtue of its assumption that the source is at a finite distance from the observer and that diffusion takes place 1sotropically in an infinite medium. (i1) In the second, the matter traversed by particles is assumed to be velocity dependent. This can be achieved by replacing the limit $x$ in Equation 3.23 by $x \beta$, where $\beta$ is the ratio of the velocity of the particle to that of light (Durgaprasad, 1967). This would imply that all particles at the point of observation have spent the same amount of time in space, leading thereby to a concept that cosmic rays were produced by a single event at a time corresponding to $t=x / \rho c$, where $\rho$ is the mean matter density in the medium. Such a hypothesis would result in temporal variations and cannot be then brought under quasi-equilibrium models.

(b) The Steady State Mode1

The steady state model assumes space-time homogenity of cosmic ray sources within the confinement volume. Under this assumption, particles have finite probability of having finite path lengths before undergoing catastrophic loss either from collision or leakage from the boundary. As discussed earlier, Equation 3.21 represents the steady state solution for cosmic ray propagation. Transforming now the energy variable to matter traversed, we can rewrite the equation as 


$$
j_{i}(E)=\frac{\rho v}{4 \pi} \int_{0}^{\infty} \frac{\left|w\left(E^{\prime}, x\right)\right|}{|w(E)|} Q^{\prime}\left(E^{\prime}, x\right) d x \exp \left[-\int_{0}^{x} d y\left\{\frac{1}{\Lambda_{1}\left(E^{\prime}, y\right)}+\frac{1}{x\left(E^{\prime}, y\right)}\right\}\right]
$$

where $Q^{\prime}\left(E^{\prime}, x\right)$ is the $x$-independent production rate of the $i^{\text {th }}$ particle defined by Equation 3.13, per gram per unit path length traversed at depth $x \mathrm{~g} \mathrm{~cm}^{-2}$, having energy $E^{\prime}$ such that it reduces to $E$ due to ionization at the point of observation; $\mathrm{X}$ is the mean matter traversed by cosmic rays corresponding to the mean residence time $\tau$. Once again, one can introduce here variations in the method of computation by making plausible assumptions regarding the energy dependence of X (Cowsik, et a1. (1967; Fichte1 and Reames, 1968; Ramaty and Lingenfelter, 1968; Shapiro, et a1. 1970a). It is also pertinent to mention here that attempts have been made recently to analyze the observed data using the diffusion model with boundary conditions (Ramaty and Lingenfelter, 1971; Pacheco, 1971).

\subsubsection{Electron Component}

In case of the electron component, almost all energy loss processes are applicable. At energies less than about a few tens of $\mathrm{MeV}$, ionization loss becomes increasingly important while at very high energies inverse Compton and synchrotron processes dominate. At intermediate energies escape loss is perhaps a more effective catastrophic energy loss than bremsstrahlung. Since the rate of energy loss due to inverse Compton scattering and synchrotron radiation are proportional to the square of the electron energy, these processes lead to a heavy drain on the energy of energetic electrons. In consequence, observations on the shape of the electron energy spectrum could lead to information on the mode of propagation of cosmic rays and the region of space where they, and hence all cosmic rays, are confined. 


\section{a) Leakage life-time model}

The simplest model, so far extensively used in all interpretative studies of the electron spectrum is to apply the leakage life time concept; accordingly Equation 3.8 can be written as

$$
\frac{\partial N}{\partial t}=Q-\frac{\partial}{\partial E}\left[-\left(a-\alpha E+b E^{2}\right) N\right]-\left[\frac{1}{\tau}+\frac{1}{T}\right] N=0
$$

Here, the positive numerical co-efficients $a$ and $b$ are related to the rate of energy loss through ionization and, synchrotron and inverse Compton processes respectively and $\alpha$ is related to the energy gain due to Fermi type of acceleration. The catastrophic losses are defined by their life times $T$ and $\tau$, for bremsstrahlung and leakage respectively. The life time against bremsstrahlung is energy independent and in the model of galactic confinement of cosmic rays $T \gg \tau$. The solution to Equation 3.28 can be written from Equation 3.21 as

$$
N(E) d E=\frac{1}{\left(a-\alpha E+b E^{2}\right)} \text { E } Q\left(E^{\prime}\right) d E^{\prime} \exp \left[-\frac{1}{\tau} \int_{E}^{E^{\prime}} \frac{d E^{\prime \prime}}{\left(a-\alpha E^{\prime \prime}+b E^{\prime 2}\right)}\right]
$$

The upper limit to this integral is either $\mathrm{E}_{s}$, the energy at which the rate of energy loss equals the rate of energy gain, or $\infty$ depending upon whether $\mathrm{E}$ is less or greater than $\mathrm{E}_{\mathrm{s}}$ respectively. It is obvious from the above solution that for $\alpha^{2}-4 a b \geq 0, N(E) d E \rightarrow \infty$ at $E_{S}=\left(a \pm \sqrt{\alpha^{2}-4 a b}\right) / 2 b$. At these energles, the rate of energy loss is equal. to the energy gain and Equation 3.28 has to be written as

$$
\frac{\partial N}{\partial t}=Q-\left(\frac{1}{\tau}+\alpha-2 b E_{s}\right) N=Q-\left(\frac{1}{\tau} \mp \sqrt{\alpha^{2}-4 a b}\right) N=0
$$

and the corresponding solution at $E=E_{s}$ is 


$$
N(E) d E=\frac{Q(E) d E}{\frac{1}{\tau} \mp \sqrt{\alpha^{2}-4 a b}} \quad 3.31
$$

The singularity in this solution occurs when the physical conditions are such that at energy $E_{s}$, the catastrophic loss of particles is same as the particle gain due to either energy loss or gain processes. Moreover, since the rate of energy loss is zero at $E_{s}$, one cannot expect steady state to prevail at this energy and one can see from Equation 3.30 that $\partial \mathrm{N} / \partial \mathrm{t}=\mathrm{Q}$ and hence $N(E) d E \rightarrow \infty$, as $t \rightarrow \infty$ at $E=E_{S}$. One can also reason from the above discussion, without going through any mathematical treatment, that if the acceleration of cosmlc rays is a continuous process like that of Fermi acceleration, then there will be an enhancement in the energy spectrum at the energy where the rate of energy loss is the same as the rate of energy gain.

Having discussed the effect of acceleration in steady state propagation of cosmic ray electrons, we shall pass on to a particular case in which $\alpha=0$ and the production spectrum is a power law of the type $Q(E) d E=\mathrm{kE}^{-\beta} \mathrm{dE}$. 
Under these conditions, one can see from Equation 3.28 that for very low energies, where Ionization loss alone is effective, the equilibrium spectrum is of the form

$$
N(E) d E=\frac{k}{a(\beta-1)} E^{-\beta+1} d E \text { for } E \ll a(\beta-1)
$$

Thus for constant energy loss, the spectrum is flatter by one power. In the energy region, where the particle loss is dominated by the leakage from the confinement volume, the equilibrium spectrum retains its spectral shape, provided the leakage is independent of energy, and from Equation 3.28 one obtains

$$
N(E) d E=k \tau E^{-\beta} d E \text { for } a(\beta-1) \ll E \ll \frac{1}{b(\beta-1) \tau}
$$

At high energies, where the energy loss due to synchrotron and inverse Compton process dominates, the equilibrium spectrum becomes

$$
N(E) d E=\frac{k}{b(\beta-1)} E^{-(\beta+1)} d E \text { for } E \gg \frac{1}{b(\beta-1) \tau}
$$

Here the spectrum is steeper by one power than the production spectrum. Thus one finds that the spectral shape changes at two characteristic energies and if such changes are detected, it. would give information on the processes responsible for the same.

b) Diffusion Mode1

Attempts have been made to determine the solution of Equation 3.8 for an arbitrary source distribution but by neglecting the catastrophic loss term $\mathrm{p}=0$ and taking only the synchrotron and inverse Compton loss processes $b(E)=-b^{2}$ (Syrovatskii, 1959; Jokipii and Meyer, 1968; Berkey and Shen, 1969; Webster, 1970; Jones, 1970; Bulanov et a1. 1972). For 
simple isotropic diffusion Equation 3.8 then reduces to

$$
\frac{\partial N}{\partial t}-\frac{\partial}{\partial E}\left(b E^{2}\right)-D \nabla^{2} N=Q
$$

When the source function $Q$ is expressed as $Q=k^{-\beta} \delta\left(t_{0}-t^{\prime}\right) \delta\left(\underline{r}_{0}-\underline{r}^{\prime}\right)$, which implies a source at a distant position $\underline{\underline{x}}_{0}$ injected an electron spectrum of the type $\mathrm{kE}^{-\beta}$ at a time $t_{0}$, the non-stationary solution at a position $\underline{r}$ and at time $t$ of Equation 3.33 can be written (Berkey and Shen, 1969) as

$$
\begin{array}{rlrl}
N(E, \underline{r}, t) & =\left[4 \pi D\left(t-t_{0}\right)\right]^{-3 / 2} \exp \left[-\frac{\left(r-E_{0}\right)^{2}}{4 D\left(t-t_{0}\right)}\right] E^{-B}\left[1-b E\left(t-t_{0}\right)\right]^{B-2} & \\
& =0 & & \text { for } E<\frac{1}{b\left(t-t_{0}\right)} \quad 3.34 \\
& & \text { for } E>\frac{1}{b\left(t-t_{0}\right)}
\end{array}
$$

Here, the first factor corresponds to the spacial dilution of particle from a point source, the second to the probability of the emitted particles to reach the point at $\underline{r}$ and the last factor gives the depreciation of the particles in an energy interval due to energy loss processes. Obviously the spectrum would show a continuous steepening as a function of energy and drop to zero at an energy $E \geqslant 1 / b\left(t-t_{0}\right)$ beyond which the particles cannot survive due to the energy loss processes during a time $\left(t-t_{0}\right)$ even if they had infinite energy to start with. If the source emits particles continuously, the steady state solution can be obtained by integrating Equation 3.34 over $t_{0}$ between the limits $t-1 / b E$ and $t$. Even here, the equilibrium spectrum terminates at energles $>1 / \mathrm{bE}$. For a source function of the type $Q\left(\underline{\underline{r}}^{\prime}, E\right)=g\left(\underline{\underline{r}}^{\prime}\right) \mathrm{E}^{-\beta}$, where $g\left(\underline{r}^{\prime}\right)$ is arbitrary source distribution, the steady state solution of Equation 3.33 
is, according to Webster (1970)

$$
N(E, r)=\iiint \frac{E^{-\beta}}{4 \pi D \mid \underline{\underline{r}-\underline{r}} T} f\left(\beta, \frac{b E\left|\underline{r}-\underline{r}^{\prime}\right|^{2}}{4 D}\right) g\left(\underline{r}^{\prime}\right) d \underline{r}^{\prime}
$$

where $f(\alpha, \beta)=\Gamma(a-1) / \sqrt{\pi} \exp (-\beta) \beta^{\frac{1}{2}} U(\alpha-1,3 / 2, \beta)$ and $U$ is the confluent hypergeometric function of the second kind. In this equation $f \rightarrow 0$, when $E \gg 4 D / b\left|\underline{\underline{r}}-\underline{\underline{x}}^{\prime}\right|^{2}$ and $\mathrm{E} \rightarrow 1$ when $\mathrm{E} \ll 4 \mathrm{D} / \mathrm{b}\left|\underline{\underline{I}}-\underline{\underline{I}}^{\prime}\right|^{2}$. One can in princlple evaluate numerically Equation 3.35 for any arbitrary source distribution with boundary conditions. We shall now take a typical case of a flat disk (like the Galaxy) of radius $\mathrm{R}$ and height $2 \mathrm{H}$, with uniform source distribution $g(\underline{r})=$ const and determine the equilibrium spectral shape at the centre of the disk $\underline{r}=0$.

For energies $E<4 D / b R^{2}$, where the energy loss is so small that the particle can diffuse through the disk with very little or no modification to its energy, the function $f$ in Equation 3.35 tends to one for all values of $\underline{r}^{\prime}$ and hence the resultant spectral shape is the same as that of the source spectrun. As the energy increases, electrons from all parts of the disk cannot reach the observer at $\underline{\underline{r}}=0$ and fewer sources contribute to the observed flux resulting in the steepening of the spectrum. Since the sources, which contribute to the flux at $\underline{I}=0$ are uniformly distributed in a thin disk, we have from Equation 3.35

$$
\begin{aligned}
& \mathrm{N}(\mathrm{E}, 0) \alpha \int_{0}^{\alpha} \frac{\mathrm{E}^{-\beta}}{4 \pi \mathrm{Dr}} \mathrm{f}\left(\beta, \frac{\mathrm{bEr}^{2}}{4 \mathrm{D}}\right) 2 \pi \mathrm{rdr} \\
& \alpha \mathrm{E}^{-(\beta+0.5)} \quad \text { for } \frac{4 \mathrm{D}}{\mathrm{bR}^{2}}<\mathrm{E}<\frac{4 \mathrm{D}}{\mathrm{bH}^{2}} \quad 3.36
\end{aligned}
$$

Here we find that the spectrum steepens by half power at an energy $E \approx 4 D / b R^{2}$, and this spectral shape continues up to an energy $E \approx 4 D / b H^{2}$ 
beyond which the sources contributing to the flux are distributed within a spherical volume of radius $\mathrm{H}$. As the energy increases even this spherical volume shrinks in size and from Equation 3.35 we obtain

$$
\begin{aligned}
& N(E, 0) \alpha \int_{0}^{\alpha} \frac{E^{-\beta}}{4 \pi D r} r\left(\beta, \frac{b E r^{2}}{4 D}\right) 4 \pi r^{2} d r \\
& \text { a } \mathrm{E}^{-(\beta+1.0)} \quad \text { for } \mathrm{E}>\frac{4 \mathrm{D}}{\mathrm{bH}^{2}}
\end{aligned}
$$

Thus at energies $\mathrm{E}>4 \mathrm{D} / \mathrm{bH}$, the equilibrium spectral shape is steeper by one power compared to the production spectrum

One can notice in the above treatment that for an axially symmetric source distribution of the type $g(\underline{r}) d \underline{r} \alpha \mathrm{rdr}$, the resultant spectral shape is steeper by half power, while for a spherically symmetric source distribution, $g(\underline{r}) d \underline{x} \propto r^{2} d r$, it is steeper by one power. These clearly indicate that the spectral shape of the equilibrium spectrum is determined by the type of source distribution. Hence Webster (1970) showed that any arbitrary break of $\Delta B$ in the spectrum can be obtained by a source distribution of the type $g(\underline{r}) d \underline{r} \alpha r^{2 \Delta \beta} d r$.

We have so far assumed that diffusion is isotropic and in an infinte medium, but introduced different source distributions with boundary conditions. It is also possible to evaluate Equation 3.33 for a finite diffusive volume with a transmission co-efficient depending upon the physical conditions, which determine the boundary, and for anisotropic diffusion (Berkey and Shen, 1969; Bulanov et al. 1972). The uncertalnties in the present experimental results do not warrant at this stage a detafled description of these complex models. However, it is easy to show (Berkey 
and Shen, 1969) that for energies $\gg 4 \mathrm{D} / \mathrm{bR}^{2}$, the boundary at $|\underline{r}|=R$

plays no role because the particle can never reach the boundary without losing much

of Its energy; on the other hand at energles $\ll 4 D / b R^{2}$, the equilibrium spectrum depends upon the boundary conditions. It is important to point out at this stage that none of the models predict an abrupt spectral steepening, and the range of energy over which the spectral change occurs Is about a decade or more in energy depending upon the boundary conditions (Webster, 1970; Jones, 1970b). 


\section{Interpretation of the observational data}

Cosmic rays we sample in the neighborhood of the Earth reach us after having undergone a chain of transformations beginning with what occurs in their source regions. Often one refers to the composiof tion/cosmic rays at the time of injection into interstellar space; on such occasions one has implicitly included modifications introduced during the process of acceleration and propagation, if any, within the source region. The interstellar injection spectrum, thus defined, undergoes further changes while the particles propagate in galactic space and attains a state of quasi-equilibrium. On entering the solar systern this equilibrium spectrum is further modulated at energies below a few $\mathrm{GeV} / \mathrm{n}$, when the particles have to work their way against the continuous outflow of solar wind from the Sun. The last phase of the journey of cosmic rays before being detected by instruments carried by balloons, rockets, and earth satellites, is their entry into the region of influence of the geomagnetic field. However, there is no real difficulty in calculating quantitatively the influence of the geomagnetic field on cosmic rays entering the magnetosphere since our knowledge of the geomagnetic field and its effect on the motion of charged particles: is now adequately understood. This knowledge then permits us to infer reliably the cosmic ray spectrum beyond the magnetosphere, i.e., in the interplanetary space, from observations made within; instruments on deep space probes, on the other hand, reveal the nature of the interplanetary cosmic rays directly. From the above brief account, it is seen that the process of deducing information on the propagation of cosmic rays in the Galaxy from earth 
based observations is a very complex one, subject to many uncertainties. In spite of this, considerable progress has been made in this field during recent years; and in what follows we shall try to deduce information on the propagation of cosmic rays by applying theoretical approaches described in the preceding section on the observational data.

\subsection{Matter traversed by cosmic rays}

During their propagation in the Galaxy, cosmlc rays interact with interstellar matter. When interactions are of electromagnetic type, the particles lose part of their energy by ionization. Nevertheless, there occur less frequent interactions which are catastrophic in nature; in these the following may take place:

(i) heavy nuclei may break up into lighter ones;

(11) at energies greater than the threshold for meson production, a11 created unstable particles w111 decay in space and give rise to stable components like electrons and photons;

(iii) the target and/or the colliding particle may be left in an excited or radioactive state, which will then decay to a stable one by the emission of particles and electromagnetic radiations; and

(iv) in case of the electron component it can suffer bremsstrahlung radiation leading to gamma ray emission.

Though in principle all these processes can lead to information on the amount of matter traversed by cosmic rays, thus far only a few could be profitably exploited; these will be reviewed now.

\subsubsection{The effect of ionization}

The rate of energy loss due to ionization of a particle, with charge Ze and velocity $B C$, in space according to Ginzburg and Syrovatskii (1964), 
is

$$
\frac{\mathrm{dE}}{\mathrm{dt}}=\frac{1.524 \times 10^{-14} \mathrm{Z}^{2} \mathrm{n}}{\beta}\left\{11 \cdot 2+\ln \left(\frac{\beta^{2}}{1-\beta^{2}}\right)-\beta^{2}\right\} \mathrm{MeV} / \mathrm{sec}
$$

where $\mathrm{n}$ is the number of hydrogen atoms $\mathrm{cm}^{-3}$. The above equation shows that the energy loss due to Ionization decreases with the Increasing veloctty, reaching a near constant value of about $4 \mathrm{Z}^{2} \mathrm{MeV}$ per $\mathrm{g} \cdot \mathrm{cm}^{-2}$ of hydrogen at relativistic energies. Since at subrelativistic energies the predominant energy drain for the nucleonic components is through lonization losses, one can in principle deduce their matter traversal by first starting with an injection energy spectrum and then comparing the spectrum modified by ionization losses, for an assumed traversal of interstellar matter, with the observed spectrum. However, in practice, this is found to be a questionable procedure since, at non-relativistic energles, the resultant spectral shape is sensitive to the propagation model employed, the form of the injection spectrum assumed, and the degree of solar modulation. It has been demonstrated quantitatively (Coms tock, 1969) that so far no single model is capable of reproducing the relative spectral shapes of the observed energy spectra of all components with a given spectral shape at injection and with the same parameters for solar modulation. In the high energy region, $\gtrsim_{5} \mathrm{GeV}$ per nucleon, where the above difficulties don't exist, the ionization loss is too small to have any observable effect on the energy spectrum. Notwithstanding all this, it has been recognized that a prior knowledge of the amount of matter 
traversed from other considerations can be used to obtain information on the mode of propagation of cosmic rays at low energies.

\subsubsection{Fragmentation of nuclei}

It stands to reason to consider that the elements and isotopes, known to be nearly absent in astrophysical objects, are also absent in cosmic ray source material. Hence these nuclei, if observed in the cosmic rays reaching the solar system, can only stem from fragmentation of heavier nuclei in collisions with matter encountered enroute (Bradt and Peters, 1950). The most striking examples of elements and isotopes, which are belfeved to be nearly absent universally and which have been extensively used in the study of matter traversed by cosmic rays, are $\mathrm{Li}, \mathrm{Be}$, and $\mathrm{B}$, and ${ }^{3} \mathrm{He}$ and ${ }^{2} \mathrm{H}$, respectively. With continuously improvIng capability and sophistication of instrumental techniques, other rare elements and isotopes are also being increasingly studied during recent years. For the reason that in this method one cannot distinguish between matter traversed in the source region and in interstellar space, we shall assume in the following general treatment that the matter traversed by cosmic rays is in interstellar space.

Before proceeding further, it is necessary to emphasize that the mean amount of matter traversed in this method also depends upon the propagation model employed because each mode1 assumes different path 
length distributions. This can be easily demonstrated by considering two extreme models, namely "the slab model" which assumes a $\delta$-function for the distribution of path lengths, and "the steady state model" which assumes an exponential distribution. For simplicity we shall consider at relativistic energies the transformation of primary nuclei of the $1^{\text {th }}$ kind to secondary nuclei of the $k^{\text {th }} k i n d\left(z_{1}>z_{k}\right)$ without taking into account the contribution from other nuclei either to $k$ or $i$. Then according to slab mode1, one gets from Equation 3.23,

$$
j_{1}(x)=j_{i}(0) \exp \left(-x / \Lambda_{1}\right)
$$

and

$$
f_{k}(x)=f_{i}(0) \cdot \frac{P_{i k}}{\lambda_{i}} \frac{\Lambda_{i} \Lambda_{k}}{\Lambda_{k}-\Lambda_{i}}\left\{\exp \left(-x / \Lambda_{k}\right)-\exp \left(-x / \Lambda_{i}\right)\right\} \quad 4.3
$$

where $x$ is the constant amount of matter traversed by the primary cosmic ray (obviously the secondaries traverse less matter); the other quantities are defined in the same manner as in section 3.4 . The ratio $R(s 1)$ of the fluxes of secondary to primary nuclei in slab model would be

$$
R(s 1)=\frac{P_{1 k}}{\lambda_{1}} \frac{\Lambda_{i} \Lambda_{k}}{\Lambda_{k}-\Lambda_{i}}\left\{\exp \left[x\left(\frac{1}{\Lambda_{i}}-\frac{1}{\Lambda_{k}}\right)\right]-1\right\} \quad 4.4
$$

On the other hand in the case of steady state models, one obtains from Equation 3.26

$$
j_{i}=\frac{\Lambda_{i} X_{1}}{\Lambda_{i}+X} ; j_{k}=\frac{\Lambda_{k} \Lambda_{1} X^{2}}{\left(\Lambda_{k}+X\right)\left(\Lambda_{1}+X\right)} \frac{P_{i k}}{\lambda_{i}} Q_{i}
$$

and

$$
R(s t)=\frac{\Lambda_{k} X}{\Lambda_{k}+X} \frac{P_{i k}}{\lambda_{i}} \quad 4.6
$$

Here $Q_{1}$ is the production rate per sr. sec. $\mathrm{g} \cdot \mathrm{cm}^{-2}$ and $X$ is the mean matter traversed by the particles if they are not attenuated by nuclear interactions. 
For a given observed value of $\mathrm{R}$, the matter traversed as estimated by these two models can be related as

$$
x=\frac{\Lambda_{k}-\Lambda_{1}}{\Lambda_{k} \Lambda_{i}} \ln \left\{\frac{\Lambda_{k}\left(x+\Lambda_{1}\right)}{\Lambda_{i}\left(x+\Lambda_{k}\right)}\right\}
$$

and for $\Lambda_{k} \simeq \Lambda_{1}$, the above equation reduces to $x=x \Lambda_{k} /\left(x+\Lambda_{i}\right)$, which shows that $x$ is always less than $X$ and the difference $(X-x)$ increases with the decreasing value of $\Lambda$ (i.e., with increasing value of charge). When $\Lambda_{i}$ and $\Lambda_{k}$ are very much larger than $x$, Equations 4.4 and 4.6 become identical and

$$
\mathrm{x} \equiv \mathrm{X}=\frac{\lambda_{i}}{\mathrm{P}_{1 \mathrm{k}}} \mathrm{R}(\mathrm{ob})
$$

The above relations can be easily generalized to take into account all fragmentation leading to the $i^{\text {th }}$ and $k^{\text {th }}$ nuclei and also for groups of nuclei.

Keeping in mind the meaning of the mean amount of matter traversed by cosmic rays, let us interpret the observations. The observed ratio of the Integral flux of 1ight nuclei $L(\mathrm{Li}, \mathrm{Be}, \mathrm{B})$ to that of medium nuclei $M$

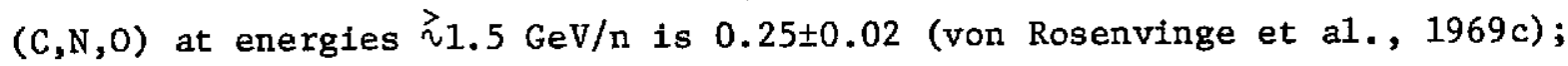
the value extrapolated to the outside of the magnetosphere is $0.23 \pm 0.02$ (Shapiro and Silberberg, 1970). One can then use appropriate cross-sections for the production of $I$ nuclei from nuclei of $z^{\geq}$(Silberberg and Tsao, $1973 \mathrm{a}, \mathrm{b})$ and by a method of successive comparison of the calculated ratio with the observed one, deduce the amount of mean matter traversed as about $4 \mathrm{~g} \cdot \mathrm{cm}^{-2}$ for the slab model and as about $6 \mathrm{~g} \cdot \mathrm{cm}^{-2}$ for the steady state model.

Likewise, $x$ and $x$ can also be deduced from the observed ratios of ${ }^{2} \mathrm{H} / \mathrm{He}$ and ${ }^{3} \mathrm{He} /\left({ }^{3} \mathrm{He}+{ }^{4} \mathrm{He}\right)$ on the assumption that ${ }^{2} \mathrm{H}$ and ${ }^{3} \mathrm{He}$ are absent in the 
source. Note that the production of these isotopes are primarily from collisions of ${ }^{4} \mathrm{He}$ nuclei with interstellar gas and that the related attenuation mean free paths are much larger than the amount of matter traversed. For these reasons, an accurate determination of these ratios above a $\mathrm{GeV} / \mathrm{n}$ would give us a more dependable value for the mean amount of matter traversed by cosmic rays although the value thus derived will again be model dependent. Unfortunately, reliable finite values for these ratios are not available so far (Gangull et al., 1967; Damle, 1968; Apparao, 1973) and the observations lead only to upper limits of $\succsim 10 \mathrm{~g} \cdot \mathrm{cm}^{-2}$ to the matter traversed.

\subsubsection{Charge composition of cosmic ray electrons}

Like in the case of universally rare nuclei, the positron component of the cosmic radiation is also expected to result from secondary processes occurring in interstellar space (also in the source regions). Since the attenuation mean free path of electrons is very large (for example, the radiation length in hydrogen is $58 \mathrm{~g} \cdot \mathrm{cm}^{-2}$ ) compared to the mean matter traversed by cosmic rays, it can be seen that in the energy region, where continuous energy losses through Ionization, Inverse Compton scattering, and synchrotron radiation do not dominate, the electron component is ideally suited for the study of matter traversed by cosmic rays. The mean matter traversed, which in this case is model independent, can be written as

$$
x=\int_{E_{1}}^{E_{2}} j_{t}(E) d E f_{o b}\left(E_{1}, E_{2}\right) / \int_{E_{1}}^{E_{2}} f_{s}(E) j_{s}(E) d E
$$

In this equation $j_{t}(E)$ is the differential flux of the total equilibrium electrons in interstellar space. One may recall here that the energy spectrum of equilibrium electrons is the same as the radio emitting electrons in the Galaxy (Section 5.3) and is not very different from that observed 
in the neighborhood of the Earth at energies $\gtrsim_{2} \mathrm{GeV}$. The quantity $\mathrm{f}_{\mathrm{ob}}\left(\mathrm{E}_{1}, \mathrm{E}_{2}\right)$ is the observed fraction of positrons within the energy interval $E_{1}$ and $E_{2}$. Similarly, $j_{s}(E)$ is the differential flux of equilibrium secondary electrons per $\mathrm{g} . \mathrm{cm}^{-2}$ of matter traversed by cosmic rays (Section 4.5 ) at energy $E ; f_{S}(E)$ is the corresponding fraction of positrons.

Equation 4.9 essentially relates the total flux of positrons in the interstellar space to its production per $\mathrm{g} \cdot \mathrm{cm}^{-2}$ of matter traversed by cosmic rays at energies of interest here. The main contribution of positrons to primary cosmic radiation comes from the decay $\pi^{+} \rightarrow \mu^{+} \rightarrow \mathrm{e}^{+}$. The charge composition of secondary electrons as a function of energy can be calculated from our knowledge of the characteristics of high energy interactions. In Figure 4.1 is shown the charge ratio $n_{e}+/ n_{e}=f /(1-f)$ as a function of energy from two such recent calculations (Ramaty and Lingenfelter, 1966a; Perola et al., 1967) above $50 \mathrm{MeV}$, where this ratio is insensitive to the propagation models. One notices that large differences exist between these calculations; these arise obviously from our incomplete knowledge of the energy spectrom of $\pi^{+}, \pi^{-}$and $\mathrm{K}$-mesons in the laboratory system over all angles of emission as a function of primary energy. However, using the existing information and the observed fraction $f_{o b}$ $(1.7 \mathrm{GeV}, 4.2 \mathrm{GeV})=0.046 \pm 018$ (Fanselow et al., 1969), one obtains values of 3.5 and $2.0 \mathrm{~g} \cdot \mathrm{cm}^{-2}$ for $x$ corresponding to Curves $I$ and II in Figure 4.1 respectively. : It needs to be pointed out here that, since the observed charge ratio for high energy atmospheric $\mu$-mesons 1 s $z 1.2$, a value close to the one according to Curve I in Fig. 4.1 (Wolfendale, 1969), the most probable value for the mean matter traversed by cosmic rays is likely to be about $3 \mathrm{~g} \cdot \mathrm{cm}^{-2}$. It is hoped that more reliable determinations in the future of the positron flux in the cosmic rays as a function of energy will lead to a better understanding of this subject. 


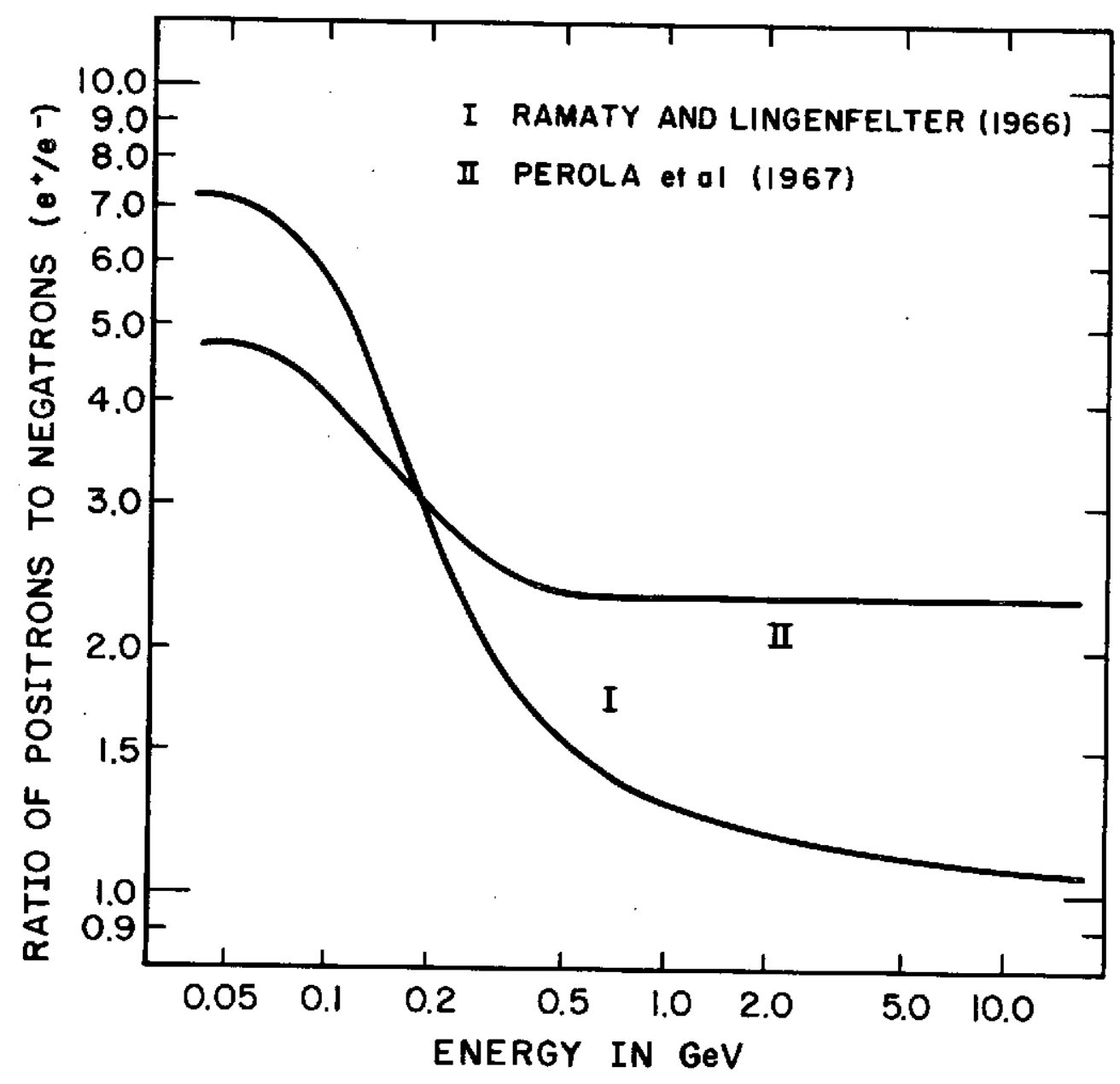

Fig. 4.2: The calculated ratio of positrons to electrons arising from nuclear collisions of cosmic rays with interstellar gas as a function of energy. 


\subsubsection{Gamma ray component of the galactic background radiation}

of the many processes which lead to the emission of background gamma rays of galactic origin through interaction of cosmic rays with interstellar matter, there are two which, from a practical point, are capable of yielding information on the gas density in the Galaxy. These are (i) bremsstrahlung of cosmic ray electrons; and (ii) decay of neutral pions produced in nuclear interactions of cosmic ray nuclei with interstellar matter. It will be shown later (Section 5.I) that the contribution of bremsstrahlung radiation is about an order of magnitude smaller than that of pionic gamma rays and, from a comparison of the observed flux of gamma rays at energies $\gtrsim 100 \mathrm{MeV}$ towards the galactic centre, one can set an upper limit of about 2 hydrogen atoms $\mathrm{cm}^{-3}$ as a mean matter density in the galactic disk.

\subsubsection{Summary}

At non-relativistic energies, where the ionization loss and solar modulation play. an important role in modifying the spectral shape of cosmic rays, it is difficult to obtain reliable information on the mean amount of matter traversed by cosmic rays from the observational data. As yet only two methods have been used with a good degree of success at relativistic energies. In the first the ratio of light to medium nuclei observed at the top of the atmosphere yields a value of about 4-6 $\mathrm{g} \mathrm{cm}^{-2}$ The second method, wherein the flux of the positron component is employed, leads to a value close to $3 \mathrm{~g} \mathrm{~cm}^{-2}$ in this the mean amount of matter traversed is not sensitive to the propagation model used. From these observations one may conclude that relativistic cosmic rays traverse a mean 
amount of 3 to $6 \mathrm{~g} \mathrm{~cm}^{-2}$ of matter in the Galaxy. It needs to be pointed out that we have so far defined the matter traversed in terms of the amount of hydrogen in $\mathrm{g} \mathrm{cm}^{-2}$; when consideration is given to the composition of the interstellar matter it is expected that it will effectively increase the amount of matter traversed as deduced above (Apparao and Ramadura1, 1967). Recent observations also suggest (Garcia-Munoz, 1973) that the mean matter traversed by cosmic rays is probably an energy dependent parameter; this aspect will be discussed separately in section 4.4 .

4.2 The chemical composition at the source

The chemical composition of the nucleonic component of cosmic rays at the time of their injection into the interstellar space would give information on the elemental abundances (the source characteristics Including nucleosynthesis), acceleration processes and injection mechanisms operative in the source regions. It is possible to deduce the source composition from the observed elemental abundances by making use of the available fragmentation cross-sections of cosmic ray nuclei and plausible models of propagation in interstellar space. Many attempts have been made in the past to determine source composition, either for groups of nuclei or for prominent elements using the slab model for the propagation of cosmic rays (Hayakawa et al., 1958; Aizu et al., 1960; Badhwar et al., 1962; Kristiansson, 1966; Beck and Yiou, 1968; Waddington, 1969); more recently it has been carried out by using different propagation models (Shapiro et a1., 1970a 1973; Ramaty and Ligenfelter, 1971; Webber et al., 1972; Casse and Goret, 1973; Cowsik and Wilson, 1973). In all these calculations, the fragmentation cross-sections used are mostly based on semi-empirical relations. We 
shall first enumerate some of the difficulties associated with this kind of work so as to make the reader aware of the uncertainties involved, before we proceed to understand the recent studies on this aspect of cosmic ray propagation.

The measurements of fragmentation cross-sections as a function of energy are still incomplete, and theoretical estimates do not cover adequately the reactions of interest. Semi-empirical relations based primarily on the work of Rudstam $(1955,1966)$ and a few modifications introduced recently (Audouze et al., 1967; Shapiro and Silberberg, 1970; Silberberg and Tsao, 1973a,b) are widely used for the purpose of computations. These relations constructed by using measured cross-sections ava11able from time to time are being constantly revised. The typical standard deviation of the calculated cross-sections from a measured value (where the latter is available), according to Silberberg and Tsao (1973a,b), varies from about $30 \%$ for target elements of $\mathrm{z} \leq 20$ to about a factor of two or more for heavy elements. Further, most of these estimates refer to interactions with hydrogen only, though helium constitutes about $10 \%$ of the atoms of interstellar gas. New observations from accelerators show that in a carbon target, the cross-section for the production of isotopes like ${ }^{9} \mathrm{Be}$ and ${ }^{10} \mathrm{Be}$ with $\alpha$-particle beams are a few times larger than the correw. sponding ones with proton beams (Fontes, et al., 1971) thereby pointing to the need to measure in the laboratory the fragmentation cross-sections of cosmic ray nuclei with helium.

The determination of the source composition of cosmic rays even at relativistic energies depends crucially on the type of propagation model employed. For example, let us consider the source abundance of iron and 
oxygen nuclel. Using first the slab model, one can write for their source ratio according to Equation 4.2:

$$
\mathrm{R}(\mathrm{Fe} / 0)_{\mathrm{s} l}=\exp \left(\mathrm{x} / \Lambda_{\mathrm{Fe}}-\mathrm{x} / \Lambda_{0}\right)\left[\mathrm{j}_{\mathrm{Fe}}(\mathrm{x}) / \mathrm{j}_{0}(\mathrm{x})\right] \quad .4 .10
$$

In the case of the steady state model, one gets from Equation 4.5

$$
R(\mathrm{Fe} / 0)_{\mathrm{St}}=\left[\left(\Lambda_{\mathrm{Fe}}+\mathrm{X}\right) /\left(\Lambda_{0}+\mathrm{X}\right)\right]\left(\Lambda_{\mathrm{Fe}} / \Lambda_{0}\right)\left[j_{\mathrm{Fe}}(\mathrm{X}) / \mathrm{j}_{0}(\mathrm{X})\right] \quad 4.11
$$

Here the quantity $\left[j_{\mathrm{Fe}}(x) / j_{0}(x)\right] \equiv\left[j_{\mathrm{Fe}}(x) / j_{0}(x)\right]$ is the observed ratio of the flux of iron nuclet to that of oxygen nuclei and $\Lambda_{\mathrm{Fe}}$ and $\Lambda_{0}$ are the attenuation mean free paths having values about $2.5 \mathrm{~g} \mathrm{~cm}^{-2}$ and $8 \mathrm{~g} \mathrm{~cm}^{-2}$ respectively; $x$ and $x$ are the mean amount of matter traversed by cosmic rays as defined by the slab and steady state models respectively. One finds from these equations that for the same observed ratio, the slab model always predicts a higher ratio of iron to oxygen nuclel at source than the steady state model. Further, as $\mathbf{x} \rightarrow \infty$, the ratio becomes infinite for the slab model while it reaches a constant value in the case of the steady state model. Taking now the ratio between Equations 4.10 and 4.11 , one gets

$$
\mathrm{R}(\mathrm{s} 1 / \mathrm{st})=\exp \left(\mathrm{x} / \Lambda_{\mathrm{Fe}}{ }^{-\mathrm{x} / \Lambda_{0}}\right) /\left[\left(\Lambda_{\mathrm{Fe}}+\mathrm{X}\right) \Lambda_{\mathrm{Fe}} / \Lambda_{0}\left(\Lambda_{0}+\mathrm{X}\right)\right] \quad 4.12
$$

which is independent of the observed ratio. Using $x=4 \mathrm{~g} \mathrm{~cm}^{-2}$ and $x=6$ thereby $\mathrm{g} \mathrm{cm}^{-2}$ respectively for the $\mathrm{s} l \mathrm{ab}$ and the steady state models,/insuring that the two models predict the same ratio of light to medium nuclei as observed (Section 4.1.2) pne gets $R(s 1 / \mathrm{st}) \approx 2$. From the above discussions, it appears that at present, the main source of error in the determination of source composition comes from the uncertainties in the propagation models and fragmentation cross-sections employed.

In Table 4.1, we show the cosmic ray source abundances relative to carbon as calculated by Shapiro et al., (1973), who made use of the 
Table 4.1

Elemental Abundance Relative to Carbon in the Source Region

\begin{tabular}{llllll} 
Element & Abundance & Element & Abundance & Z & Abundance \\
\hline $\mathrm{H}$ & $5 \times 10^{5}$ & $\mathrm{Si}$ & $200 \pm 30$ & $40-44$ & $6.2 \pm 3.3 \times 10^{-3}$ \\
$\mathrm{He}$ & 26000 & $\mathrm{~S}$ & $30 \pm 6$ & $50-54$ & $1.0 \pm 0 \cdot 3 "$ \\
$\mathrm{C} *$ & 1000 & $\mathrm{Ar}$ & $7 \pm 5$ & $55-59$ & $8.2 \pm 2.9 \times 10^{-4}$ \\
$\mathrm{~N}$ & $110 \pm 20$ & $\mathrm{Ca}$ & $22 \pm 8$ & $75-79$ & $9.7 \pm 1.5 "$ \\
$\mathrm{O}$ & $1070 \pm 20$ & $\mathrm{Cr}$ & $3 \pm 3$ & $80-84$ & $4.1 \pm 1.0 "$ \\
$\mathrm{Ne}$ & $160 \pm 20$ & $\mathrm{Fe}$ & $210 \pm 30$ & $85-90$ & $2.9 \pm 1.2 "$ \\
$\mathrm{Na}$ & $8 \pm 4$ & $\mathrm{Ni}$ & $8 \pm 2$ & $90-94$ & $4.9 \pm 1.2 "$ \\
$\mathrm{Mg}$ & $230 \pm 20$ & $\mathrm{Cu}-\mathrm{Se}$ & $0.61 \pm 0.22$ & $\geq 95$ & $4 \pm 4 \times 10^{-5}$ \\
$\mathrm{Al}$ & $20 \pm 10$ & $\mathrm{Br}-\mathrm{Y}$ & $.01 \pm .004$ & & \\
\hline
\end{tabular}

* Normalization 
steady state model with $x=5 \mathrm{~g} \mathrm{~cm}^{-2}$. These abundances are in agreement with those calculated independently by other researchers using the same model (Ramaty and Lingenfelter, 1971; Webber et al., 1972; Casse and Goret, 1973). The abundances given in this Table 4.1 assume a power law spectrum in energy per nucleon, but if the spectrum obeys a power law in rigidity, then the value for hydrogen would be $2 \times 10^{4}$ instead of $5 \times 10^{4}$. It has been observed that there is a striking simflarity of the abundances of elements in solar cosmic rays to those in the photosphere of the Sun, thereby implying that, whatever it may be, the process of acceleration has no charge dependent preference (Biswas and Fichtel, 1965). If this argument is extended to galactic cosmlc rays, then their source composition derived above can be related to the features of the chemical composition of sources which generate cosmic rays. On this account, a comparison with the solar abundances can yield some interesting results on the origin of these elements.

The ratio (CRS/SS) of the abundances of cosmic ray source elements to those of solar system relative to iron is plotted in Figure:4.2: as a function of atomic number (Shapiro et a1., 1973). In this plot, the solar abundances for neon and sulphur are taken from the measurements of solar flare particles (Bertschi et al., 1973; Mogro-Campero and Simpson, 1972a,b; Crawford et al., 1972; Teegarden et al., 1973b). This figure reveals that while cosmic ray sources seem to be under-abundant in elements lighter than neon, there is a striking similarity with solar abundances above neon. This prompted Silberberg et al. (1973) and Kozlovsky and Ramaty (1973) to suggest that all elements in cosmic ray sources are produced through explosive nucleosynthesis (Arnett, 1969; Truran and Arnett, 1970; Arnett 


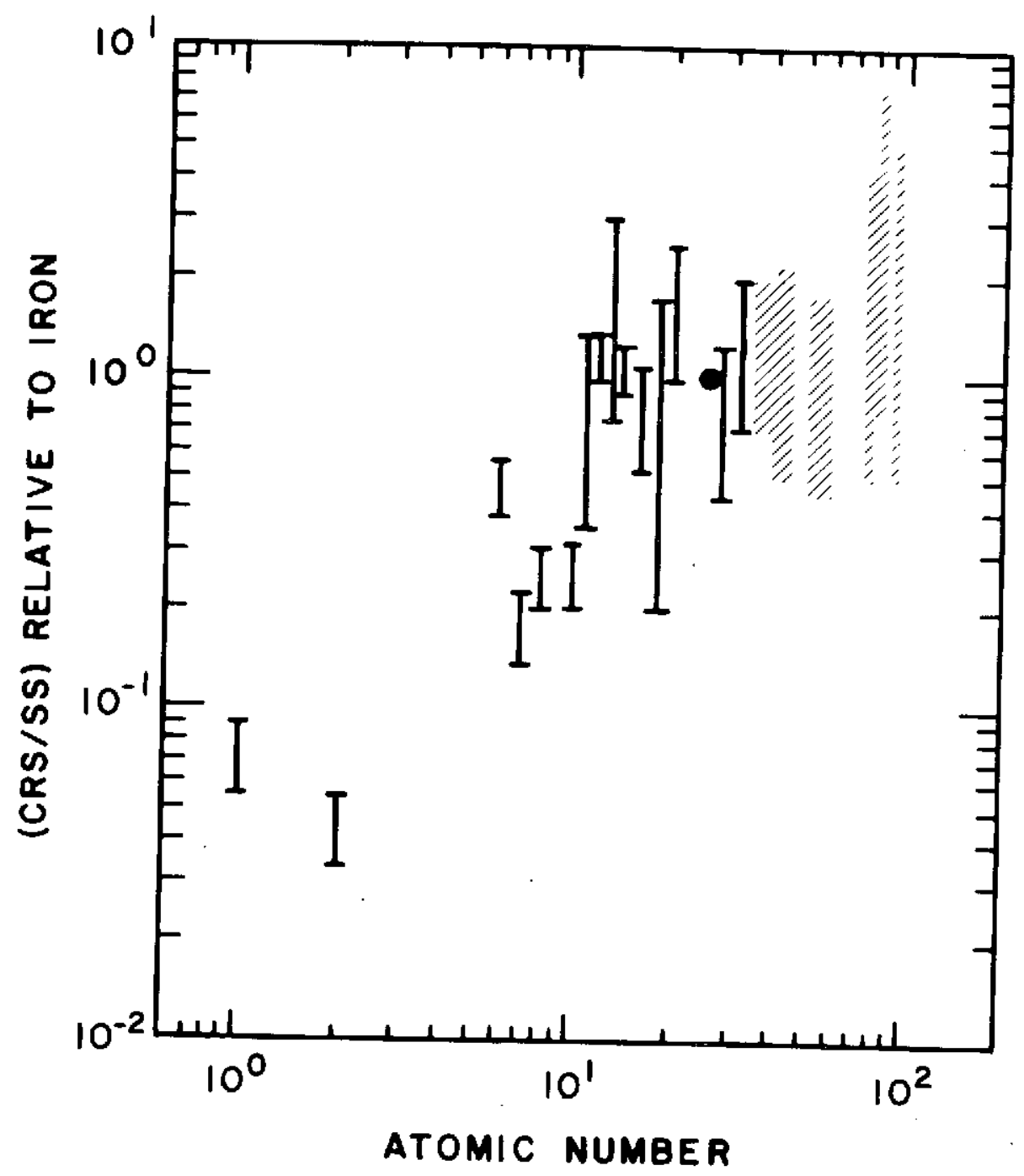

Fig. 4.2: The ratio of cosmic ray source abundance to solar system abundance as a function of atomic number, relative to iron, which is
shown without errors. 
and Clayton, 1970) and the enrichment of solar system abundances of elements 1ighter than neon can come from other processes such as hydrostatic helium burning (Clayton, 1968). The under-abundance of hydrogen and helium in cosmic ray sources resembles that expected for materials ejected from supernovae (Truran and Cameron, 1971).

Contrary to the above point of view, it has been argued by many that cosmic rays are not directly associated with violent events. It was suggested by Bradt and Peters (1950) that a selective electromagnetic acceleration process is necessary to account for the high abundance of heavy nuclei in cosmic rays; one can therefore examine whether this is related to Ionization properties. In Figure. 3. are plotted the ratio (CRS/SS) as a function of the first ionization potential of each element up to nickel; this plot exhibits a possible correlation that the relative cosmic ray abundances decreases as the first lonization potential increases (Kristiansson, 1971, 1972; Havnes, 1971, 1973; Casse and Goret, 1973). Havnes (1973) proposed that rotating magnetic stars could be the possible sources of cosmic rays, which can accelerate interstellar matter with a selective acceleration dependent upon the ionized state of the interstellar gas around the star. Cowsik (1971) suggested a preferential injection mechanism for heavy elements into the accelerating regions due to the low equilibrium charge state of heavy elements compared to their rigidity (Korchak and Syrovatskil, 1958), the observed 1ncrease of the iron to helium ratio in large solar flares at small energies (Price et al., 1971a) has also been attributed to this mechanism. Nevertheless, because of the large errors in the ratios (CRS/SS), it is difficult at present to distingulsh between explosive nucleosynthesis with further 


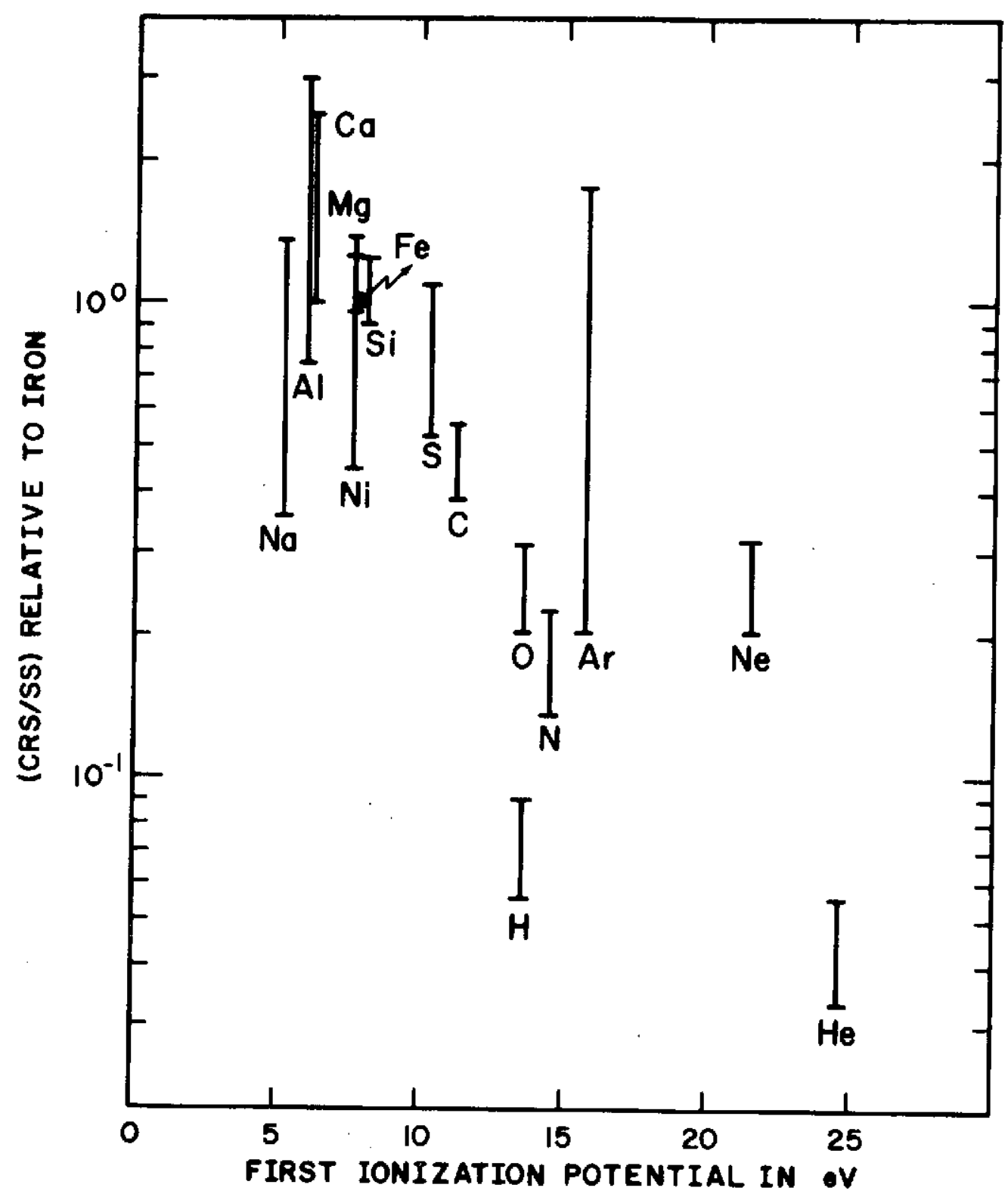

Fig. 4.3: The ratio of cosmic ray source abundance to solar system abundance as a function of the first lonization potential of each element, relative to iron. 
acceleration and possible preferential acceleration mechanisms.

The study of abundances of cosmic ray nuclei of charge $>30$ gives a clue to the process of nucleosynthesis. The abundance of ultra heavy nuclel at source given in Table 4.1 is based on the estimates of Fowler (1973) who made use of all the existing observations (Price et al., 1971b; O'Sullivan et al., 1971; Shirk et al., 1973; Blanford et al., 1973a,b; Binns et a1.. 1973; Fowler et al., 1973). These abundances seem to be In better agreement with r-process than with the total solar abundances relative to Iron (Fowler et al., 1970; Price et al., 1971b); however, a more reliable distinction can be made only when individual abundances are measured, because in some of the charge groups used in Table 4.1 only a few elements, depending upon the process of nucleosynthesis, are expected to dominate the observed flux (Seeger et al., 1965). It is also found that the ratio $\mathrm{Pb} / \mathrm{U}$ at the top of the atmosphere is about 1.3 , indicating thereby that the UH nuclei are young.

Before closing this section, let us briefly examine the possibility of obtaining some information on the propagation models from the source composition. As we have mentioned earlier that the slab model would predict a source abundance for Iron a factor of two larger than the steady state model; at the same time, the slab model cannot explain the observed abundance of ultra heavy nuclei (Fowler et al., 1970; Cowsik et a1., 1970). Ramaty and Lingenfelter (1971) have made an extensive study of the various propagation models such as simple 3-dimensional diffusion with boundary conditions, compound diffusion and an infinite medium with exponential escape time. In doing this, these authors find that the observed abundances of fragmentation products from lithium to iron can 
be understood provided the following conditions are satisfied in the respective models: (1) for simple diffusion with absorbing boundaries, the scattering mean free path $\lambda_{s}$ cannot be larger than $0.1 \mathrm{pc}$; (i1) for simple diffusion with reflecting boundaries, the amount of fragmentation depends principally on the escape probability at the boundary and is nearly independent of the scattering mean free path with the escape life time $t_{e} \sim 1.5 \times 10^{7}$ yrs; (iif) for compound diffusion, the characteristic scale length in the 3-dimensional random field $\lambda_{\mathrm{m}} \approx \lambda_{\mathrm{p}} \approx 30 \mathrm{pc}$ (Section 3.3) with $t_{e} \sim 2 \times 10^{7}$ yrs; and (iv) for an exponential path length the value of $t_{e} \approx 3 \times 10^{6}$ yrs corresponding to $x \approx 5 \mathrm{~g} \mathrm{~cm}^{-2}$. Obviously, there is no clear justification to choose or to reject any one of these models on the basis of elemental abundances alone when there is a wide choice of physical parameters that can be varied.

\subsection{Modification of the energy spectrum at low energies}

It is found that the energy spectrum of cosmic ray nuclei observed in the terrestrial vicinity can be described by a simple power law at energies greater than a few $\mathrm{GeV} / \mathrm{n}$. Below this energy, the spectrum deviates from a simple power law such that the differential intensity first increases slower with decreasing energy, reaches a maximum at around a few hundred $\mathrm{MeV} / \mathrm{n}$, and then decreases at still lower energies; the spectral shape below a few $\mathrm{GeV} / \mathrm{n}$ is not the same for all components. This change in the spectrun can result from the combined effect of solar modulation, energy loss in interstellar space during propagation, and perhaps to some extent from differences in the spectra at infection. In 
this section we consider only the effect of cosmic ray. propagation in Interstellar space for different models using various injection spectra, and compare the results with observations.

It can be shown from Equation 3, 19 that in the energy fogion where (a) continuous energy loss processes are elther negligible or proportional to energy and (b) catastrophic loss processes are independent of energy, the shape of the energy spectrum of cosmic rays, when expressed as a power law in kinettc energy per nucleon, does not change during the propagation. This condition is satisfied by the nucleonic components at relativistic energies where the ionization loss is negligible, and the nuclear interaction cross-sections and escape from the Galaxy are reasonably independent of energy. At these energies experimental observation cannot distinguish between power law spectra either in rigidity $j(R) \propto R^{-} \beta$ or in kinetic energy per nucleon $j(E) \propto E^{-\beta}$ or in total energy per nucleon $j(W) \propto W^{-\beta}$. Since the source spectrum at low energles is rather difficult to deduce directly from observations, one is tempted to assume that the spectral shape at high energies continues right down to the lowest energies. In Figure 4. A are shown the power aw spectra in kinetic energy per nucleon (Curve E), rigidity (Curve R), and total energy per nucleon (Curve W) plotted in the kinetic energy per nucleon scale for the same constant of proportionality. One can see from this figure that while these three forms of spectra become indistinguishable at high energies, they differ significantly at low energies. Currently, there is no apriori reason to prefer any one of these spectra over the others because each one has its own claim to be chosen: (i) the rigidity spectrum may 


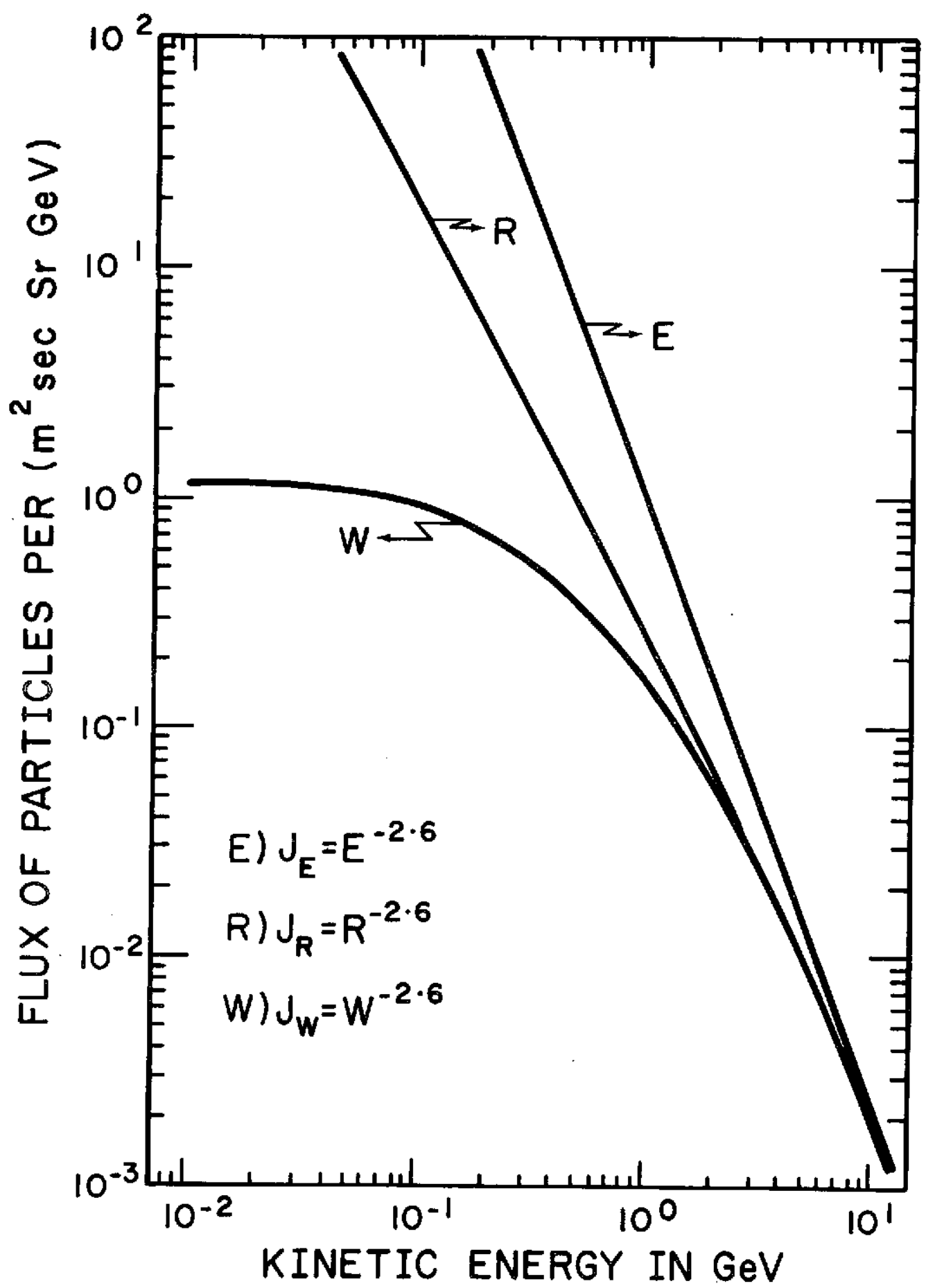

FIg. 4.4: The proton intensity as a function of kinetic energy, plotted in arbitrary units, for three different types of power law spectra. 
result from acceleration and storage by the magnetic fields in the source region (Kaplon and Skadron, 1966); (ii) the shockwave acceleration in supernovae can lead to a power law in kinetic energy per nucleon (Co1gate, 1968); and (iii) the Fermi-type of acceleration (Fermi, 1949) can lead to a power law in total energy per nucleon. We shall now at tempt to bring out the merits and demerits of these three source spectra as we analyze the observed spectra of nuclei.

The effect of Ionfzation on the energy spectrum of cosmic rays in space can be well exemplified by starting with a power law spectrum in kinetic energy at the time of particle injection into interstellar space. The results are shown in Figure 4.5 for the spectral change of the different nuclear components by making use of the steady state model with $\mathrm{X}=$ $6 \mathrm{~g} \mathrm{~cm}^{-2}$ (solid curves) and the slab model with $\mathrm{x}=4 \mathrm{~g} \mathrm{~cm}^{-2}$ (dotted curves). Curve $I$ in this figure is the injection spectrum; Curves $\mathrm{p}, 0$, and $F e$ are typically the equilibrium spectra of hydrogen, oxygen, and iron nuclei, which are normalized to the given injection spectrum around $3 \mathrm{GeV} / \mathrm{n}$; the $\mathrm{Y}-\mathrm{scale}$ in this figure is expressed in arbitrary units. One can see from this figure that the effect of interstellar ionization losses is to reduce the flux at low energies; in the case of the steady state model, this implies that low energy particles come from nearby sources. It also becomes clear from this figure that the slab model suppresses the flux of particles of low energy more than the steady state model and this effect becomes more pronounced as the particle charge increases. 


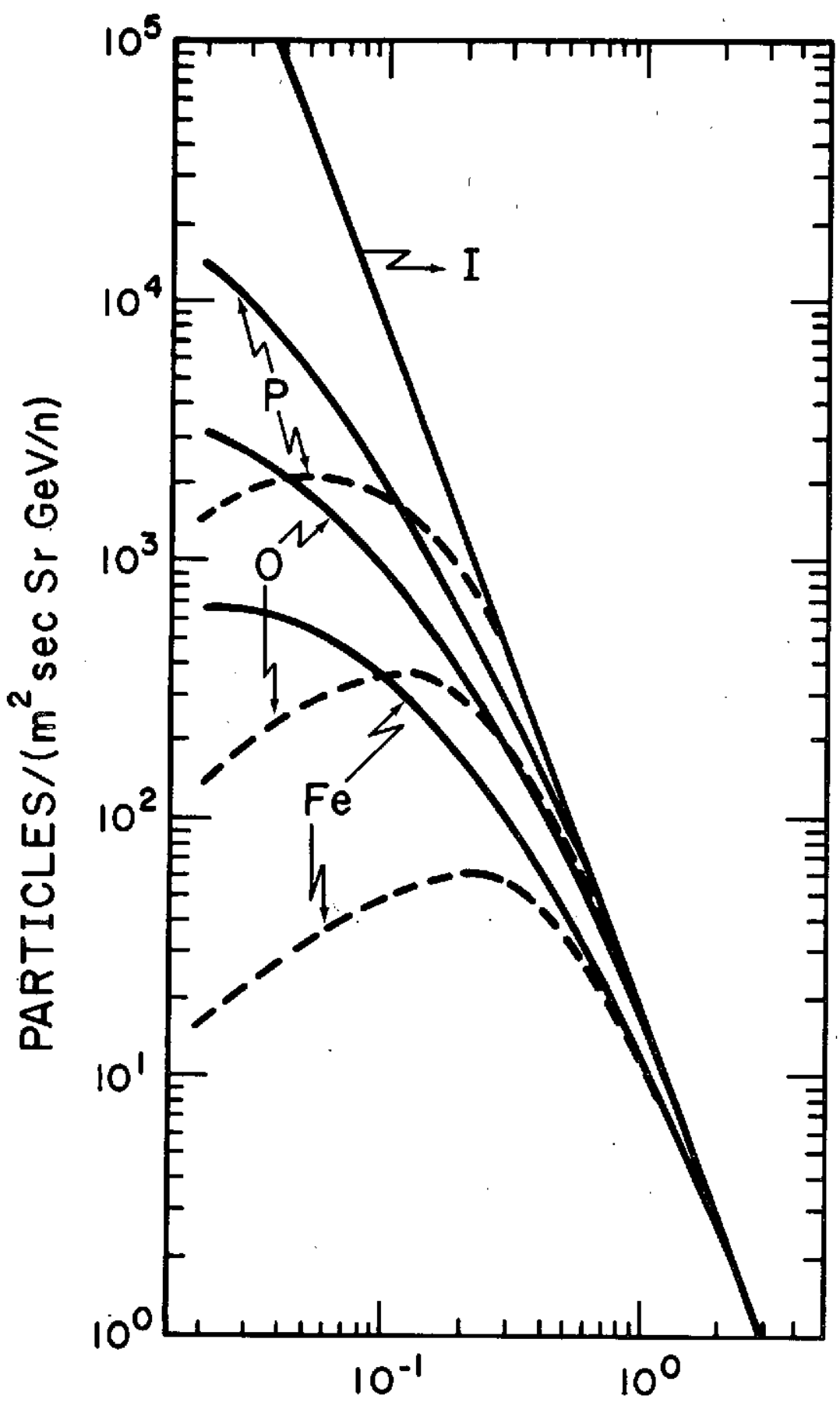

\section{KINETIC ENERGY IN GeV/n}

Fig. 4.5: The effect of ionization during propagation is shown using a steady state model with $X=6 \mathrm{~g} \mathrm{~cm}^{-2}$ (solid curves) and slab model with $x=4 \mathrm{~g} \mathrm{~cm}^{-2}$ (dotted curves) for a power law spectrum at injection (Curve I); Curves $\mathrm{P}, \mathrm{O}$ and $\mathrm{Fe}$ are for protons, oxygen and iron nuclei respectively. 
In FIgure $(4.6$ are shown. the equilibrium spectra of hydrogen and helium nuclel in interstellar space for three different types of infection spectra. In getting these results, the steady state model has been used with $X=6 \mathrm{~g} \mathrm{~cm}^{-2}$. Curves $\mathrm{E}, \mathrm{R}$ and $\mathrm{W}$ correspond to the power law infection spectra in kinetic energy per nucleon, rigidity and total energy per nucleon respectively, which are normalized at 5 GeV with the observed spectra (curves 0 ) during the period of minimum solar modulation. It is apparent from this figure that if the equilibrium spectrum in kinetic energy exists below $100 \mathrm{MeV} / \mathrm{n}$, in the same manner as indicated by this figure, the cosmic ray energy density in interstellar space would continue to increase as the energy decreases; however, this behavior is not exhibited by other spectra. The attempts made so far to deduce information on the spectral shape of cosmic rays at injection using different modulation theories yielded one conclusive resultgnamely the source spectrum is closer to a power law in total energy per nucleon (Meyer, 1971; Comstock et al., 1972; Ramadurai and Biswas, 1972) or a rigidity spectrum which flattens below about $1.5 \mathrm{GV}$ (Burger, 1971). However, none of these analyses could reproduce the entire spectral shape of the observed protons and helium nuclei right from about $10 \mathrm{MeV} / \mathrm{n}$ to a few $\mathrm{GeV} / \mathrm{n}$; this can, perhaps, be due to our incomplete knowledge of the solar modulation and/or the intrinsic nature of source spectra these nuclei have at the time of injection. Nevertheless, solar modulation does not significantly affect the ratio fluxes of of jelements as a function of energy ; in what follows we briefly review the work carried out so far in this direction. 


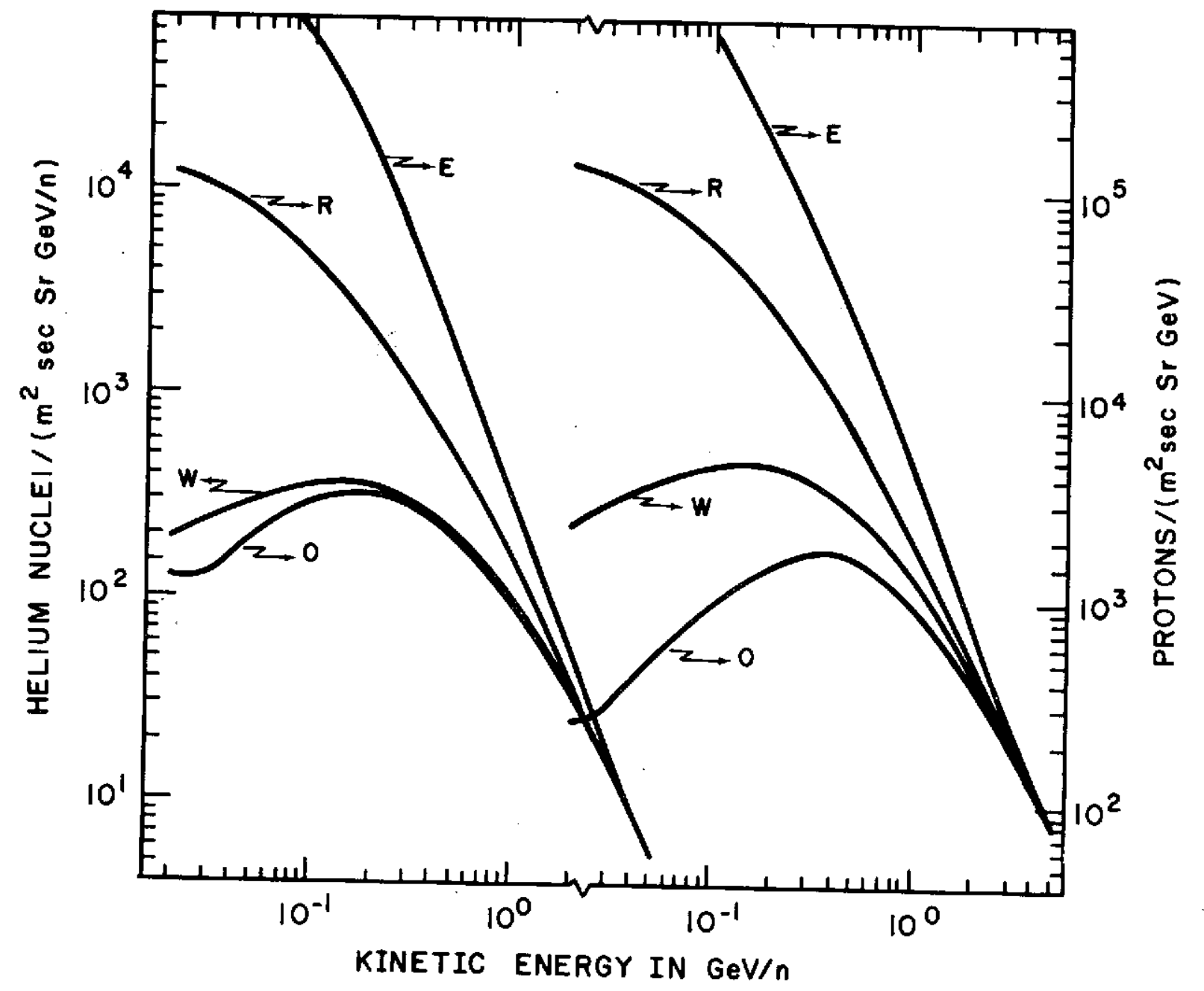

Fig. 4.6: Equilibrium spectra of protons and helium nuclei in interstellar space, using steady state model with $X=6 \mathrm{~g} \mathrm{~cm}^{-2}$ for different injection spectra shown in Figure 4.5 (Curves $E$, $R$ and $W$ ), are compared with the observed spectra during the perlod of minimum solar modulation (Curve 0 ). 


\subsubsection{L/M Ratio}

Since the light nuclei $L(L i, B e, B)$ in cosmic radiation are produced mainly by the fragmentation of medium nuclei $M(C, N, 0)$, their ratio of intensities as a function of energy has been extensively made use of to understand the propagation of cosmic rays in the Galaxy (Badhwar and Daniel, 1963; Blswas, et al., 1966; Cowsik, et al., 1967; Fichtel and Reams, 1968; von Rosenvinge, et al., 1969b; Gloeckler and Jokipii, 1970; Syrovatski1 and Kuzhevsky, 1970; Garcia-Munoz and Simpson, 1970; Shapiro, et al., 1970b; Pacheco, 1971). In Figure 4,7, we have; shown the observed ratio, $4 / M$ as a function of energy (Anand, et al., 1969b; Freier, et al., 1966; Hagge, et al., 1968; Malmquist, 1967; Garcia-Munoz and Simpson, 1970; Reams and Fichtel, 1968; von Rosenvinge, et al., 1969b; Webber and Ormes, 1967; Mason, 1972) along with the theoretical estimates. In this figure, Curve $A$ is the calculated ratio using steady state model with $X=6$ $\mathrm{g} \mathrm{cm}^{-2}$ for a power law spectrum in rigidity at injection (Tandon, 1970) and Curve $B$ is for a power law spectrum in total energy per nucleon (Mason, 1972). Notice that while Curve A is clearly in disagreement with the observations at low energies, Curve B too fails to give adequate agreement with the data points; it can also be shown that the calculated ratio using slab model as we1l as Gaussian distribution for path lengths would be close to Curve B (Ramadura1, 1970). It is thus seen that a single power law in rigidity as a source spectrum is incompatible with the observations and that either there could be an interference of a second component at energies below a few hundred $\mathrm{MeV} / \mathrm{n}$, or the adiabatic deceleration in the solar wind becomes important below about $200 \mathrm{MeV} / \mathrm{n}$. 


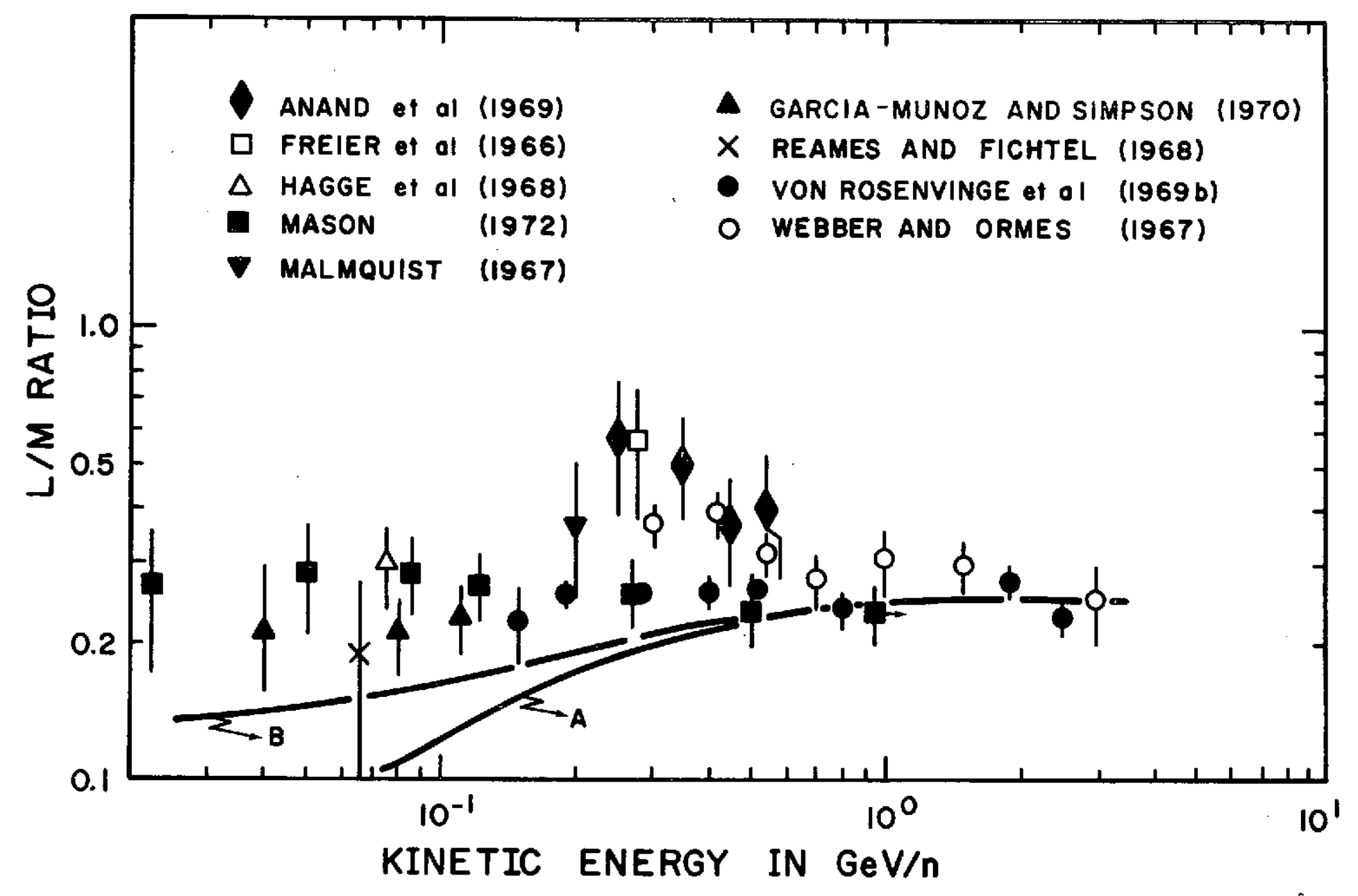

Fig. 4.7: The ratio of light to medium nuclei as a function of kinetic energy per nucleon. Curves $A$ and $B$ are the calculated ratios in interstellar space using steady state model for power law spectra in rigidity and total energy per nucleon respectively, at infection. 


\subsection{2 $2 \mathrm{H} /{ }^{4} \mathrm{He}$ Ratio}

This ratio is insensitive to solar modulation since the charge to mass ratio of ${ }^{2} \mathrm{H}$ and ${ }^{4} \mathrm{He}$ is the same, and the observed ratio as a function of energy should reveal some aspects of interstellar propagation of cosmic rays. ${ }^{2} \mathrm{H}$ nuclel are primarily produced during collisions of $\mathrm{p}+{ }^{1} \mathrm{H}, \mathrm{p}+$ ${ }^{4} \mathrm{He}$, and $\alpha+{ }^{4} \mathrm{He}$. Many theoretical estimates of the ratio ${ }^{2} \mathrm{H} /{ }^{4} \mathrm{He}$ are now avallable as a function of energy (Meyer, et a1., 1968; Ramaty and Lingenfelter, 1969; Hsieh and Simpson, 1969; Comstock, et a1., 1972; Ramadurai and Biswas, 1972). In these calculations one may have to take into account at low energies the effect of elastic collisions toward the redistribution of energy of the colliding and target particles (Ramadurai and Biswas, 1972). In Figure 4,8, we display the recent measurements; of the ratio ${ }^{2} \mathrm{H} /{ }^{4} \mathrm{He}$ (Hurford, et al., 1973; Teegarden, et al., 1973b) along with those summarized by Hsieh, et al. (1971); the year of measurement is also shown against the authors. Curves $A$ and $B$ in this figure are the interstellar ratios estimated by Meyer (1971) using steady state model with $X=6.3 \mathrm{~g} \mathrm{~cm}^{-2}$ for a source spectrum of the type $\left(E+E_{0}\right)^{-2.6}$, where $E_{o}=$ $500 \mathrm{MeV}$ and 0 respectively; Curves $\mathrm{C}$ and $\mathrm{D}$ are for steady state model with $\mathrm{X}=7 \mathrm{~g} \mathrm{~cm} \mathrm{~cm}^{-2}$ for a source spectra of the type $\mathrm{W}^{-2.6}$ and $\mathrm{R}^{-2.6}$ respectively (Biswas andiRamgdurat, 1971). Prior to the 1972 data, it was concluded by Simpson (1971) that the observations imply a power law in total energy per nucleon at injection. However it is evident from Figure 4.8: that: the ratios measured during 1972 at energies below $50 \mathrm{MeV} / \mathrm{n}$ are lower than the earlier ones by about an order of magnitude, 


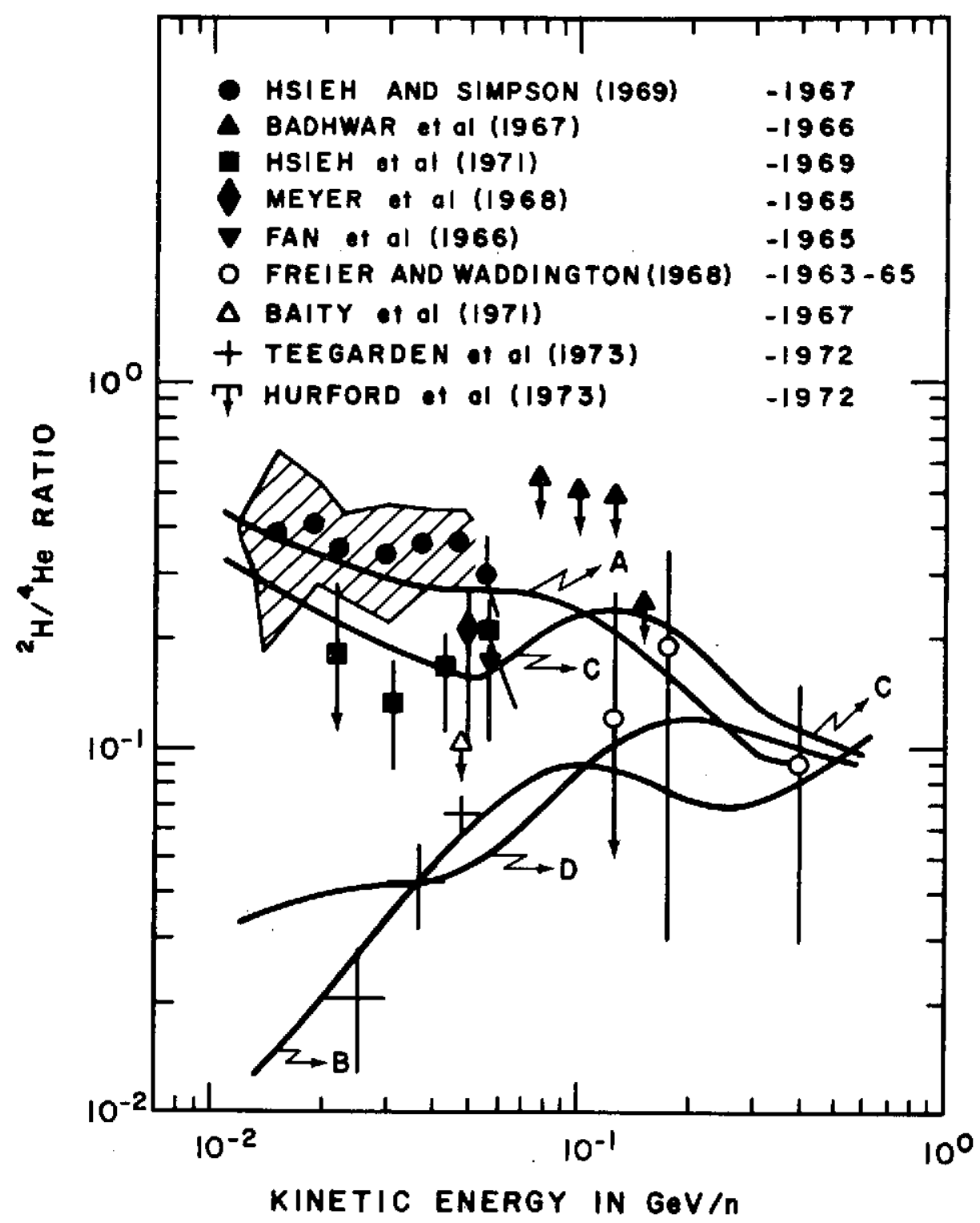

Fig. 4.8: The ratio of deutrons to helium nuclel as a function of kinetic energy per nucleon; theoretical estimates of this ratio in interstellar space shown by solid curves are all on the basis of steady state model for different injection spectra as described in the text. 
suggesting thereby that either the source spectrum is close to a power law in kinetic energy per nucleon without much adiabatic deceleration, or there could be a significant enhancement in the interstellar ${ }^{4} \mathrm{He}$ at low energy, which is noticeable at $1 \mathrm{AU}$ even with adiabatic deceleration and without a corresponding increase in ${ }^{2} \mathrm{H}$ (Stone, 1973).

\subsubsection{He/4 He Ratio}

Since the mass to charge ratios of these two nuclei are different, also

their solar modulation will/be different. This aspect was first employed by Biswas et al (1967) to determine the modulation parameters. In Figure 4.9 the observed ratio of .. He $\%^{4}$ He 1 s shown as complled by Hsieh and Simpson (1970) along with the calculated ratio at $1 \mathrm{AU}$, after correcting for solar modulation, including adiabatic deceleration as a function of energy. Curve $A$ in this figure is the calculated ratio using a Gaussian path length distribution (Section 3.5.1) with $\langle\mathrm{x}\rangle=4 \mathrm{~g} \mathrm{~cm}^{-2}$ and a Fermi spectrum (Biswas and Ramadurai, 1973); eurve $B$ is that using steady state model with $X=4 \mathrm{~g} \mathrm{~cm}^{-2}$ and a power law in total energy per nucleon (Comstock et al, 1972). One can see from this figure that it is difficult to distinguish between the two models of propagation employed in the calculations because of the effect of adiabatic deceleration below about $200 \mathrm{MeV} / \mathrm{n}$.

\subsubsection{He/M Ratio}

In Figure 4010, the observed ratio of the helium to medium nuclei as a function of energy is shown (Balasubrahmanyan, et a1, 1966; Comstock et al., 1969; Fan, et al., 1968; Hagge, et al., 1968; Mason, Garcia1972; Munoz and Simpson, 1970; Teegarden et al., 1970; von Rosenvinge, et al., 1969c). In this figure curve $A$ is the estimated ratio using 


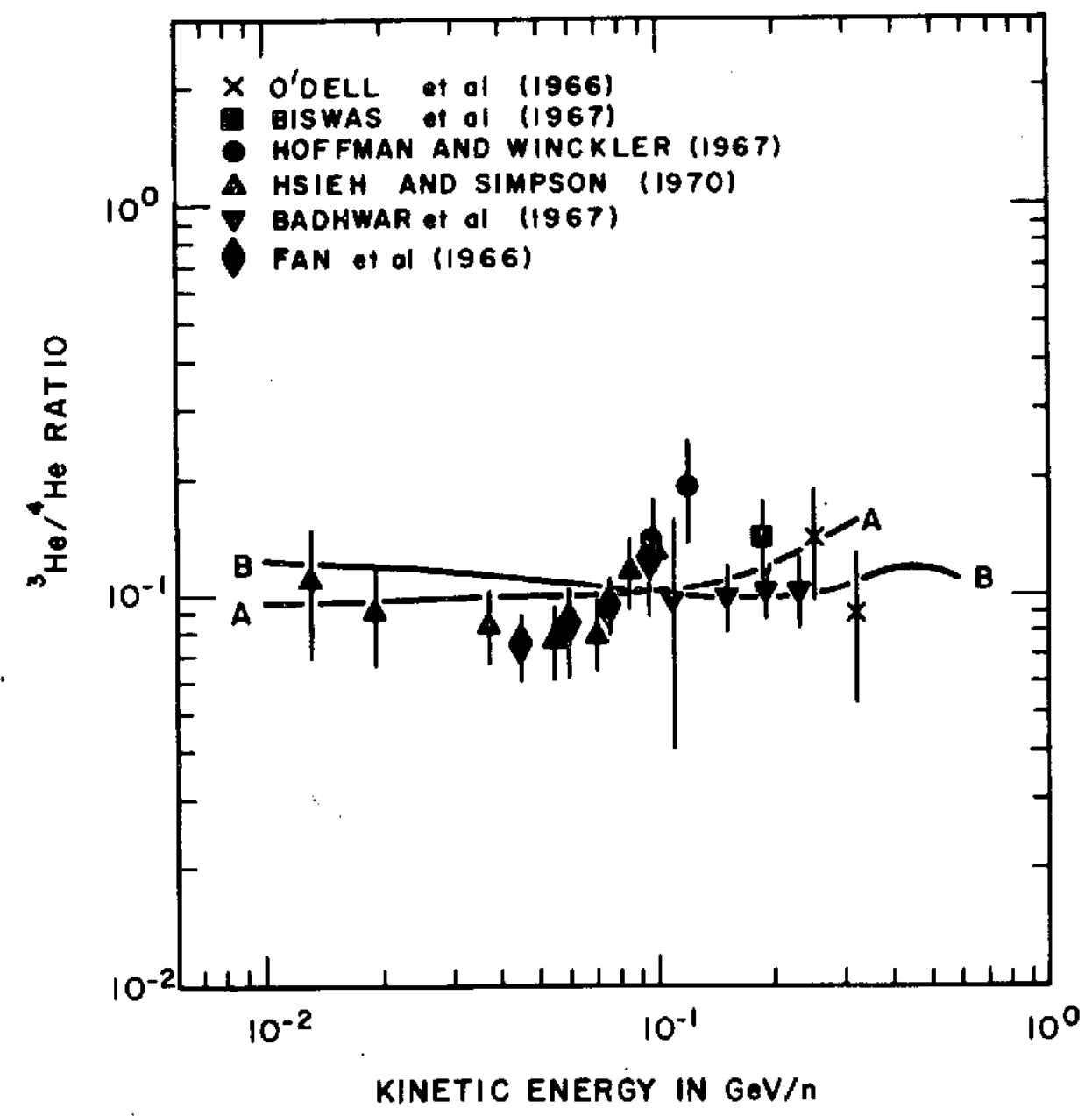

Fig. 4.9: The ratio of helium isotopes as a function of kinetic energy per nucleon. Curves $A$ and $B$ are the estimated ratios at 1 AU (after correcting for adiabatic deceleration in the solar system), using Gaussian distribution and steady state model respectively. 


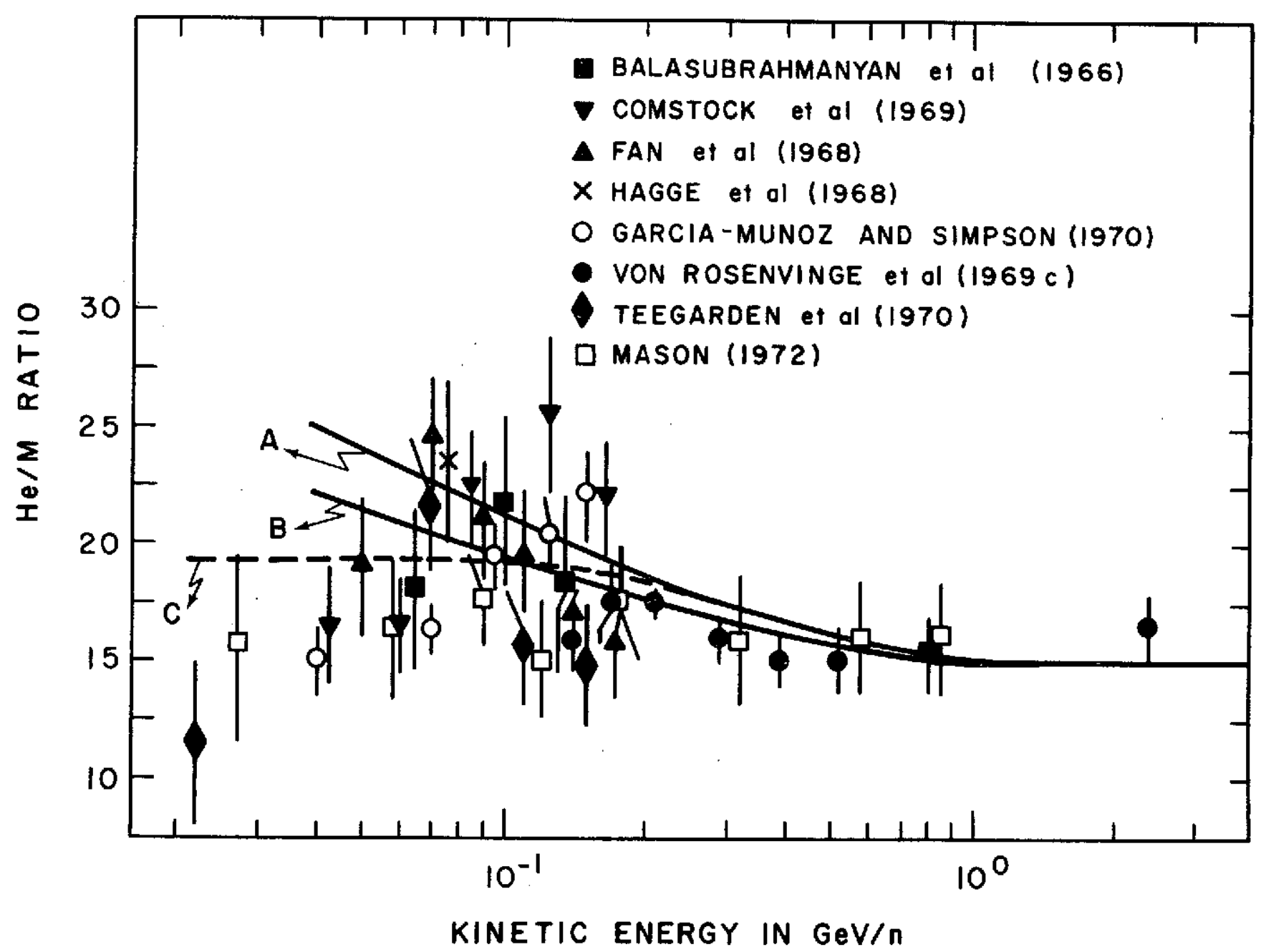

Fig. 4.10: The ratio of helium to medium nuclei as a function of kinetic energy per nucleon. Curves $A$ and $B$ are the expected ratios in interstellar space on the basis of Gaussian distribution and steady state model respectively; Curve $\mathrm{C}$ is the expected ratio at 1 AU from Curve A. 
a Gaussian distribution of path lengths with $\langle x\rangle=4 \mathrm{~g} \mathrm{~cm}^{-2}$ for a power law in total energy per nucleon (von Rosenvinge, et a1., 1969c) and curve $B$ is the expected ratio on the basis of steady state model with $\mathrm{X}=7 \mathrm{~g} \mathrm{~cm}^{-2}$ (Tandon, 1970); curve $\mathrm{C}$ is the modulated spectrum from curve A (Mason, 1972) using adiabatic deceleration below $200 \mathrm{MeV} / \mathrm{n}$. The trend in the data points in this figure below $100 \mathrm{MeV} / \mathrm{n}$ indicates an enhancement of medium nuclet with respect to helium.

\subsubsection{He/LH Ratio}

It has been shown earlier (Figure 4.5) that the effect of lontzation increases with increasing charge and as a result, the ratio of helium to heavy nuclei should be sensitive to propagation models at low energies. In Figure 4.11, we. have shown the observed ratio of helium nuclei to light heavy nuclei LH $(10 \leq \mathrm{Z} \leq 15)$ as a function of energy (Reams and Fichte1, 1967; Fan, et al., 1968; Comstock, et al., 1969; von Rosenvinge et al., 1969c), along with the theoretical estimates; here curves $A$ and $B$ are as described in Section 4.3.4. One notices from this figure that it would be rather difficult to explain these observations on the basis of propagation model with Gaussian distribution for path lengths (curve A) even after correcting for adiabatic deceleration.

\subsubsection{He/VH Ratio}

Figure $4: 12$ highlights the ratio of helium nuclei to very heavy nuclei $\mathrm{VH}(20 \leq \mathrm{z} \leq 28)$ as a function of energy, : where the data points are from the works of Reams and Fichtel (1968), Comstock, et a1., (1969) and von Rosenvinge et al., (1969c); between 60 and $90 \mathrm{MeV} / \mathrm{n}$, the data shown is deduced from the recent work of Price, et al., (1973). In this figure curves $A$ and $B$ are the calculated ratio as described in 


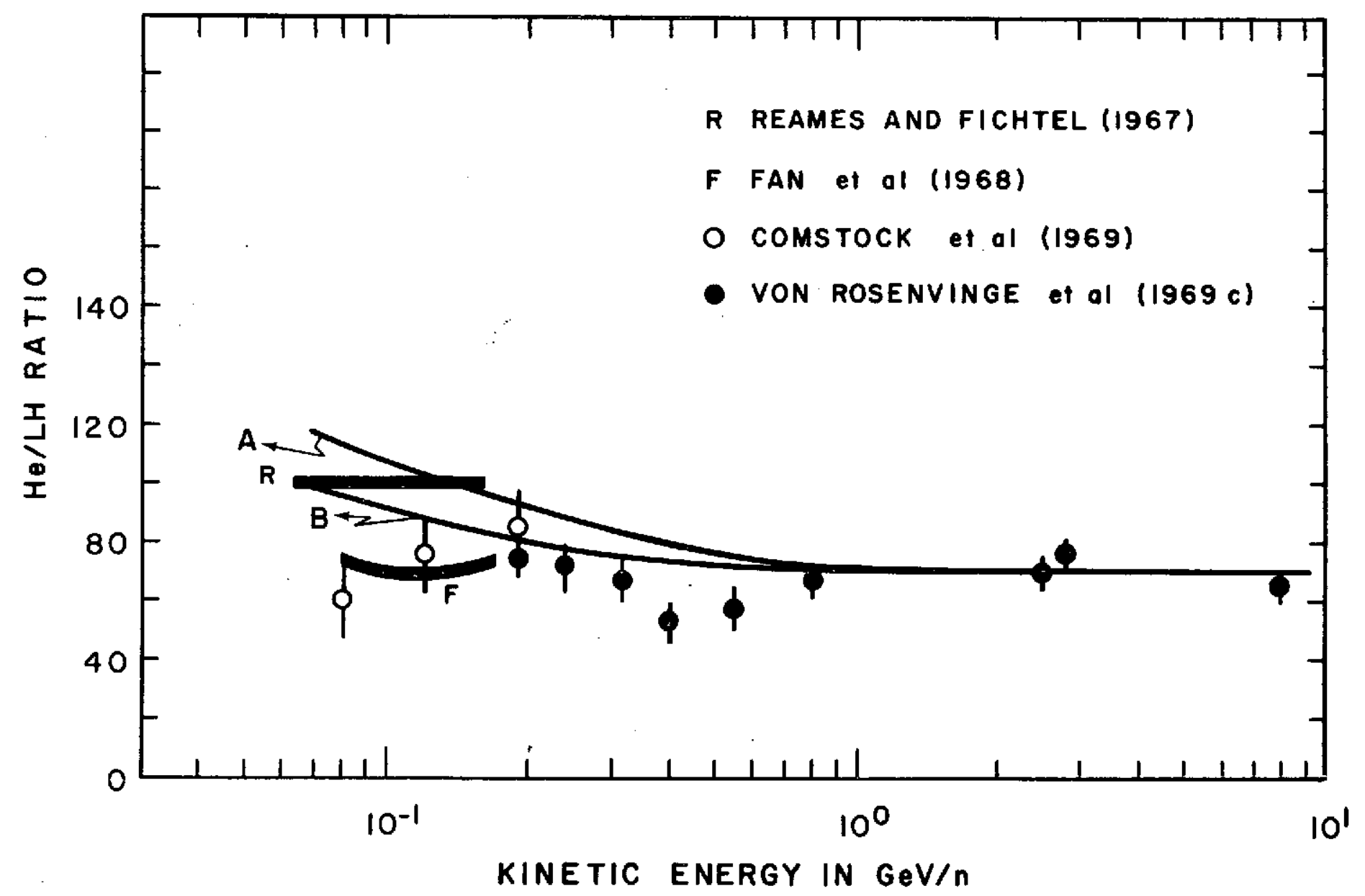

Fig. 4.11: The ratio of helium to light heavy nuclei as a function of kinetic energy per nucleon; the expected ratios in interstellar space are as shown in the same manner as in Figure 4.10. 


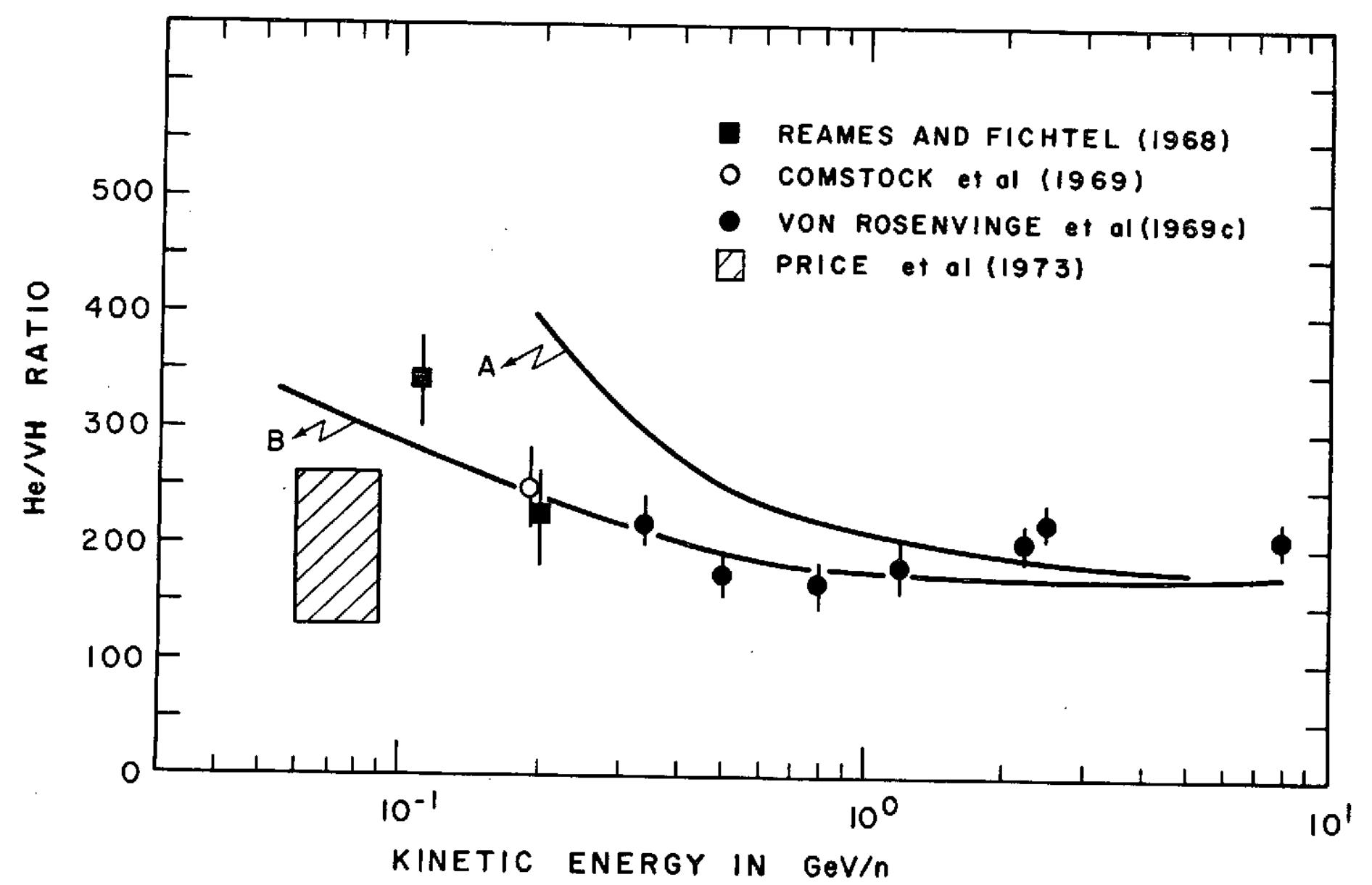

Fig. 4.12: The ratio of helium to very heavy nuclei as a function of kinetic energy per nucleon; Curves $A$ and $B$ are the expected ratios similar to those in Figure 4.10. 
section 4.3 .4 and 1 t becomes quite obvious here that the Gaussian path length distribution (curve A) is incompatible with the observations.

\subsubsection{Sunmary}

From the above studies, the following conclusions $c$ an be drawn without much ambiquity

(i) The source spectrum for cosmic rays cannot be a simple power law either in rigldity or In kinetic energy per nucleon, over the entire energy range so far covered. It is seen that, while a simple power law in total energy per nucleon is quite consistent with all the observations described so $f a r$, one may have to express the source spectrum in terms of a rigidity spectrum with a desired flattening below about $1.5 \mathrm{GV}$ to match the above requirements, so that the interstellar electron spectrum can also be well explained by the same spectrum (Section 4.5).

(ii) The steady state model consistently explains almost all observations better than the other models of propagation.

(iii) It becomes quite evident from Figures 4.7 to 4.13 that the observations below about $100 \mathrm{MeV} / \mathrm{n}$ cannot be understood purely by propagation effects in interstellar space; a similar inference is also obtained from a study of $\mathrm{C} / 0$ and $\mathrm{N} / 0$ ratios (von Rosenvinge, et al., 1969b; Bhatia, et a1., 1970). These anomalles can be accounted if the adiabatic deceleration in the solar neighborhood anounts: to about $200 \mathrm{MeV} / \mathrm{n}$ (Goldsteln et al, $1970 ;$ Gleeson and Uhrch, 1971), though one cannot rule out the possibility of the 
low energy cosmic rays having a different origin (Biswas, et al., 1966; Comstock, 1969; F1chte1 and Reames, 1968).

(iv) The observed turn up of the spectrum below a few tens of $\mathrm{MeV} / \mathrm{n}$ (Mogro-Campero, et al., 1973; Price, et al., 1973; Hovestädt, et al. 1973; McDonald, et al., 1974) and the anomalous charge composition Hovestadt, et a1., 1973; McDonald, et a1., 1974) cannot be understood at present by interstellar propagation and solar modulation described above, without invoking a new component having a different chemical composition. 


\subsection{Modification of energy spectrum at high energies}

In the preceeding sections, we Implicitly assumed for simplicity in all the calculations, in order to understand the observational data described so far, that (1) the energy spectra of all components of cosmic rays are similar at injection and hence an energy independent source composition, (ii) the path-length distribution is identical for all components and, (iii) the matter density is constant over the entire storage volume, where the observed cosmic rays propagate. The preceeding analyses did indicate that perhaps, not all these assumptions are valid, though at low definitive

energies it is difflcult to arrive at any. $f$ conclusion due to the effect of energy loss processes in interstellar space and solar modulation. At high energies, where the above difficulties do not exist, it is possible to obtain information on these aspects directly from observations. Indeed, the recent experimental investigations using different experimental techniques (Julibsson et al., 1972; Juliusson and Meyer, 1973; Smith et al., 1973a; Balasubrahmanyan and Ormes, 1973; Ormes and Balasubrahmanyan, 1973; Brown et al.,1973b; Webber et a1., 1973b) clearly indicate a change in the charge composition of cosmic rays at high energies. In this section we briefly present these experimental data and critically examine all the theoretical explanations put forth so far to understand these observations.

4.4.1 Observed change in the abundances of secondary nuclei

Any change in the abundance of secondary nuclel produced in interstellar space with energy can give some information on the possible dependence of propagation parameters on energy. In Figure 4.13 are plotted the ratio of intensities of $\mathrm{Li}, \mathrm{Be}, \mathrm{B}$ and $\mathrm{N}$ to $\mathrm{C}$ and 0 , at the top of 


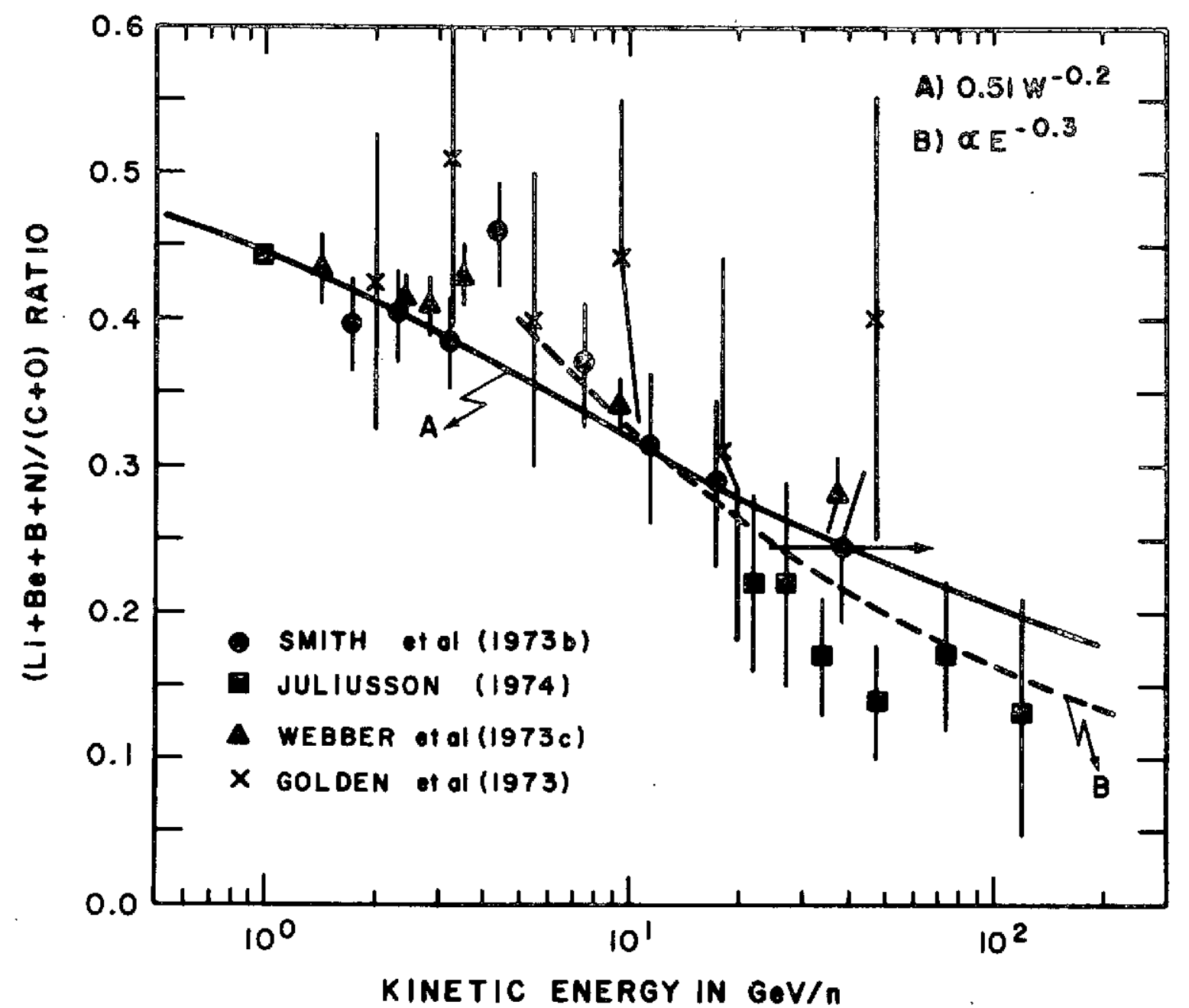

Fig. 4.13: The ratio of secondary nuclei to $\mathrm{C}+\mathrm{O}$ as a function of kinetic energy per nucleon; Curves $A$ and $B$ are two possible dependents of this ratio, one on total energy and the other on kinetic energy respectively. 


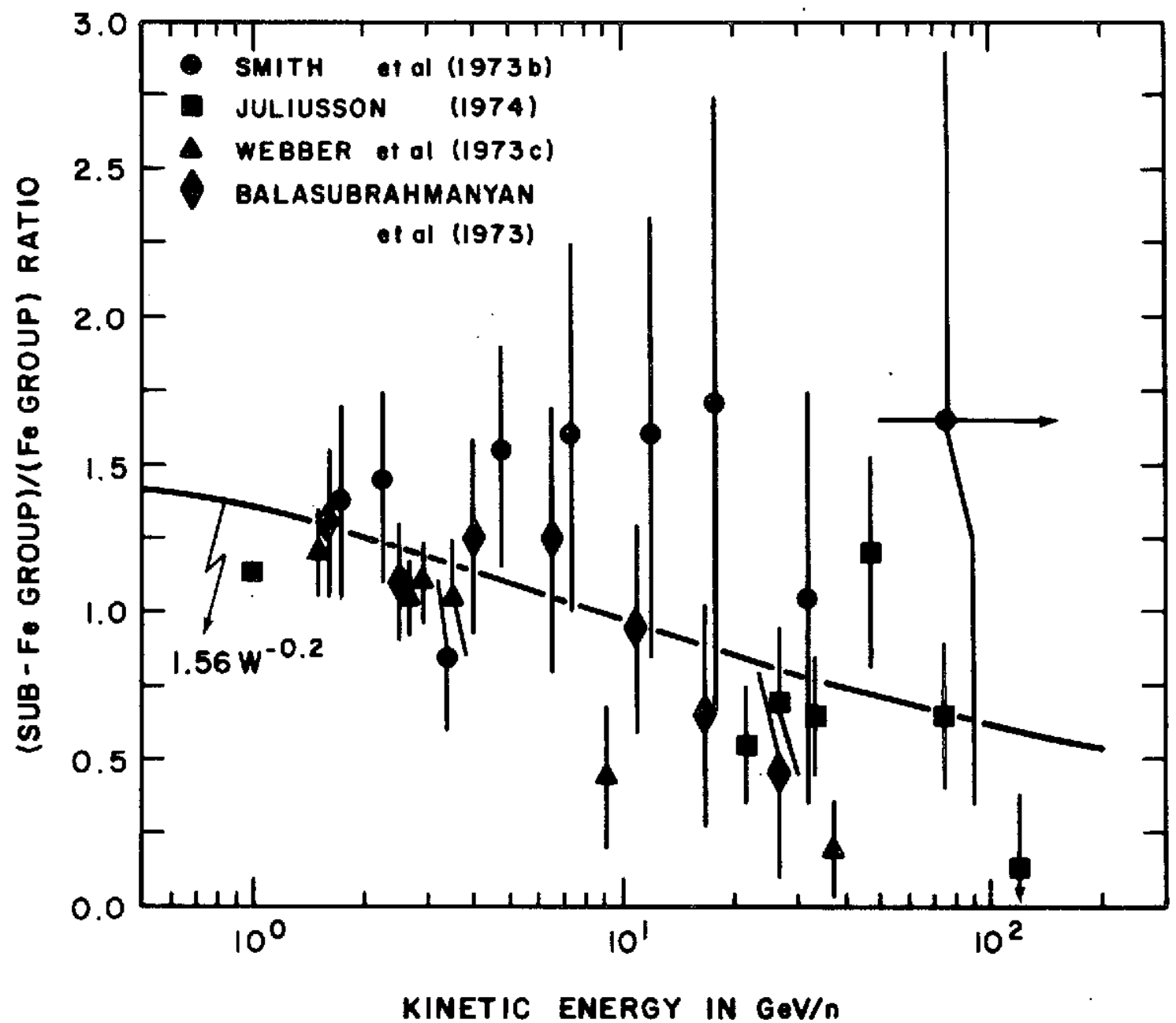

Fig. 4.14: The ratio of secondary nuclei to tron group as a function of kinetic energy per nucleon; curve shown here is similar to that of Curve A in Figure 4.13 . 
the atmosphere, as a function of kinetic energy per nucleon as summarized by Garcia-Munoz (1973) (Smith, et al., 1973b; Juliusson, 1974; Webber, et a1., 1973c); included also by crosses are the results from Golden, et al. (1973). For comparison, we have also shown in this figure two types of energy dependence, one varying as $\mathrm{W}^{-0.2}$ (Curve $A$ ) and the other varying as $\mathrm{E}^{-0.3}$ (Curve B) above $5 \mathrm{GeV} / \mathrm{n}$. From this figure one can notice that, except for the results of Golden, et al. (1973), the data points above 5 $\mathrm{GeV} / \mathrm{n}$ indicate an energy dependence as strong as that of Curve $\mathrm{B}$. In Figure 4.14, the observed ratios of sub-iron group to iron-group of nuclei are shown as a function of energy as given by Garcia-Munoz (1973). In this figure, different authors (Smith, et al., 1973b; Juliusson, 1974; Webber, et a1., 1973c; Balasubrahmanyan, et a1., 1973) have used different criteria in defining the charge groups and the observed scatter of points does not permit any definitive conclusions to be drawn as in the case from F1gure 4.13; : for compartson we have also drawn a a $\mathrm{w}^{-0.2}$. Direct measurements of the spectral indices of nuclei are given in Table 4.2 from the work of Smith, et al. (1973a), Balasubrahmanyan and Ormes (1973), and Juliusson (1974), who have made use of power law spectra in rigidity, kinetic energy per nucleon, and total energy per nucleon, respectively. Again one notices from this table that the secondary nuclei are steeper than their primaries and their indices increase by about 0.2 between about a few $\mathrm{GeV} / \mathrm{n}$ and $\sim 100 \mathrm{GeV} / \mathrm{n}$.

It has been pointed out recently by Webber, et a1. (1973c) that a stronger dependence of the type $\alpha E^{-0.5}$ is operative right down to 100 $\mathrm{MeV} / \mathrm{n}$, in contradiction to their previously reported results summarized In Figures.4.7 to 4.12. However, Price, et al. (1973) have observed no such 
Table 4.2

Observed Spectral Indices of Cósmic Ray Nuclel

\begin{tabular}{|c|c|c|c|c|c|c|}
\hline \multirow[b]{2}{*}{ Elements } & \multirow{2}{*}{$\begin{array}{l}\text { Berkeley* } \\
\mathrm{R}>8.5 \mathrm{GV} / \mathrm{C}\end{array}$} & \multirow[b]{2}{*}{ Elements } & \multirow{2}{*}{$\begin{array}{c}\text { GSFC** } \\
\mathrm{E}>3.3 \mathrm{GeV} / \mathrm{n}\end{array}$} & \multirow[b]{2}{*}{ E1ements } & \multirow{2}{*}{$\begin{array}{l}\text { ChIlcagot } \\
W>1.5 \mathrm{GeV} / \mathrm{n}\end{array}$} & \multirow{2}{*}{$\mathrm{W}>20 \mathrm{GeV} / \mathrm{n}$} \\
\hline & & & & & & \\
\hline $\mathbf{P}$ & $2.63 \pm .08$ & $\mathbf{P}$ & $2.75 \pm .03$ & $\mathrm{LI}$ & $2.95 \pm .12$ & $2.9 \pm .8$ \\
\hline $\mathrm{He}$ & $2.47 \pm .03$ & He & $2.77 \pm .05$ & $\mathrm{Be}$ & $3.09 \pm .14$ & $3.2 \pm .7$ \\
\hline Li & $2.67 \pm .13$ & LI & $2.28 \pm .15$ & B & $2.95 \pm .07$ & $2.8 \pm .4$ \\
\hline $\mathrm{Be}$ & $2.66 \pm .12$ & $\mathrm{Be}$ & $2.6 \pm .2$ & $\mathrm{C}$ & $2.65 \pm .02$ & $2.9 \pm .2$ \\
\hline B & $2.76 \pm .08$ & B & $2.76 \pm .13$ & $N$ & $2.74 \pm .03$ & $3.1 \pm .3$ \\
\hline $\mathrm{c}$ & $2.54 \pm .04$ & $\mathrm{C}$ & $2.52 \pm .06$ & 0 & $2.53 \pm .02$ & $2.6 \pm .1$ \\
\hline $\mathbf{N}$ & $2.72 \pm .09$ & $\mathrm{~N}$ & $2.73 \pm .11$ & $\mathrm{Ne}$ & $2.57 \pm .03$ & $2.9 \pm .2$ \\
\hline 0 & $2.52 \pm .05$ & 0 & $2.57 \pm .06$ & $\mathrm{Mg}$ & $2.56 \pm .03$ & $2.7 \pm .2$ \\
\hline $\mathrm{Li}, \mathrm{Be}, \mathrm{B} \& \mathrm{~N}$ & $2.72 \pm .07$ & $\mathrm{~B} \& \mathrm{~N}$ & $2.77 \pm .08$ & $\mathrm{Si}$ & $2.50 \pm .03$ & $2.2 \pm .2$ \\
\hline $\mathrm{C \& O}$ & $2.53 \pm .03$ & $\mathrm{C \& O}$ & $2.56 \pm .04$ & S & $2.6 \pm .1$ & -- \\
\hline \multirow[t]{4}{*}{$9 \leq Z \leq 14$} & $2.52 \pm .07$ & $10 \leq Z \leq 14$ & $2.44 \pm .07$ & Ar & $2.5 \pm .1$ & -- \\
\hline & & $15 \leq Z \leq 23$ & $2.1 \pm .2$ & $\mathrm{Ca}$ & $2.7 \pm .1$ & $2.5 \pm .3$ \\
\hline & & & & $\mathrm{Cr}$ & $2.6 \pm .1$ & -- \\
\hline & & $\mathrm{Fe}$ & $2.0 \pm .14$ & $\mathrm{Fe}+\mathrm{Mn}$ & $2.39 \pm .04$ & $2.2 \pm .2$ \\
\hline
\end{tabular}

* for power law spectra in rigidity (Smith et al., 1973a)

** for power law spectra in kinetic energy per nucleon (Ormes and Balasubrahmanyan, 1973; Balasubrahmanyan and Ormes, 1973)

+ for power law spectra in total energy per nucleon (Juliusson, 1974) 
variation from about $2 \mathrm{GeV} / \mathrm{n}$ down to $30 \mathrm{MeV} / \mathrm{n}$. Thus one can infer from Figures 4,13 and 4.14 and Table 4.2 that the furrent observations suggest that the ratio of secondary nuclei to primary nuclei varies efther as $W^{-\alpha}$ right from the smallest energies or as $E^{-\alpha}$ only above $\sim 5 \mathrm{GeV} / \mathrm{n}$, with $\alpha \simeq 0.2 \pm .1$. This observed dependence would directly lead to the energy dependence of matter traversed by cosmic rays and, perhaps, the possible energy dependent confinement of cosmic rays either in the source or in the storage volume.

\subsubsection{Energy dependence in the abundance of primary nuclef}

The study of the change of abundance of primary nucle1 as a function of energy can be used to examine whether 1 ts consequences are internally consistent with the propagation effects derived from the observed energy dependence of the ratio of secondary to primary nuclei; it can also give some additional information on source spectra. In Figure 4.15 are plotted the data on the ratio $(\mathrm{C}+\mathrm{O}) /(\mathrm{Fe}-\mathrm{group})$ as a function of energy from the observations summarized by Garcia-Munoz (1973), after making necessary corrections to define the Iron group as $\mathrm{Z}>24$. Likewise, in Figure.4.16, the intensity ratios of LH nuclel $(10 \leq Z \leq 14)$ to the Fe-group are plotted as a function of energy (Atallah, et a1., 1973; Balasubrahmanyan, et al., 1973; Juliusson, 1974); shown in these figures by shaded regions are the expected ratios in the source reglons from Table 4.1. One may notice that there is an indication for the ratios to decrease with increasing energy and attain values close to the source ratios at high energies; this trend is also indicated in Table 4.2 , where there is an observable change in the spectral indices with charge. 


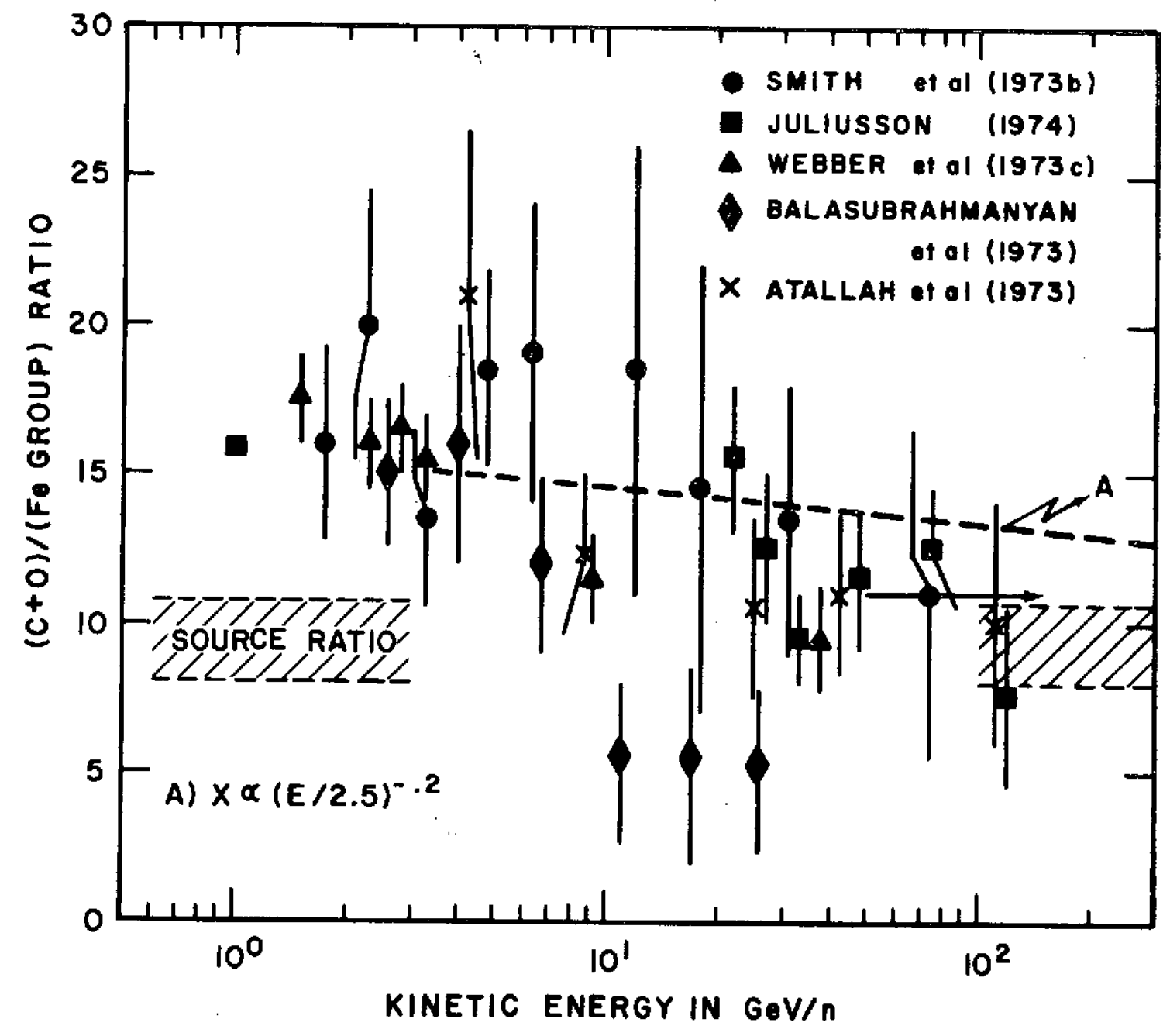
$A$ is the expected ratio on the basis of steady state model using an energy dependence of the type shown in Figure 4.14. 


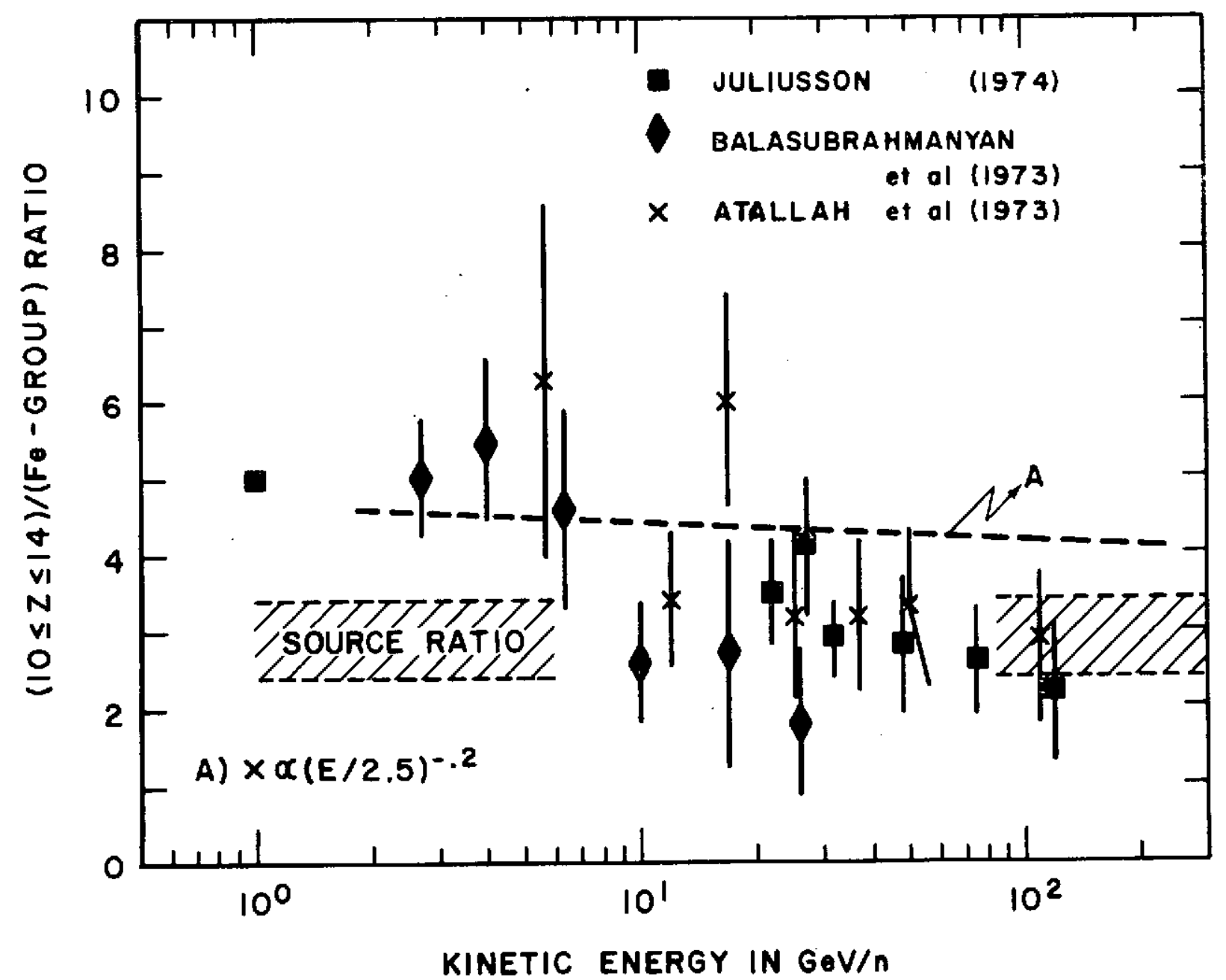

Fig. 4.16: The ratio of light heavy nuclei to iron group as a function of kinetic energy per nucleon; Curve $A$ is the expected ratio similar to that in Figure 4.15 . 
It is, however, realized that the present status on observational data is to some extent unclear. For example, the observed ratio of (C+0)/(Fe-group) by Balasubrahmanyan, et al. (1973) are smaller than those obtained by other authors and are lower than the source ratio above $10 \mathrm{GeV} / \mathrm{n}$. In contrast to this, Golden, et al. (1973) found no such variation with energy and the ratio (LH/Fe-group). Furthermore, it has been pointed out by Juliusson (1974) that the $\mathrm{C} / 0$ ratio decreases at energies greater than $20 \mathrm{GeV} / \mathrm{n}$; the observed decrease is from about 1.1 around a few $\mathrm{GeV}$ to about 0.7 at $\sim 100 \mathrm{GeV} / \mathrm{n}$, while the source ratio is about 0.9 . Badhwar and Osborn (1973) found a similar trend while studying the ratio of integral fluxes of $\mathrm{C}$ and $\mathrm{O}$ at energies $>23 \mathrm{GeV} / \mathrm{n}$. If these findings are confirmed by future experiments, it will imply that the source spectra are not the same for all nuclet!

\subsubsection{Theoretical models to explain the change in composition}

If one accepts for the moment the correctness of the observational findings for possible energy dependence of the charge composition of cosmic rays, described above, they may be interpreted in many ways. There is no experimental evidence so far to suggest that the interaction mean free paths of nucleonic components increase with energy, in the energy range of a few GeV/n to $\sim 100 \mathrm{GeV} / \mathrm{n}$; in consequence, many of the explanations proposed so far have questioned the basic assumptions made in the beginning of Section 4.4 on propagation studies. These theories can be generally distinguished on the basis of either energy dependent galactic confinement of cosmic rays or energy dependent confinement in special regions within the galactic space,such as sources or clouds. In this section we briefly review these ideas and examine them critically. 
(i) Energy dependent galactic confinement of cosmic rays

noticed

We have already $/$ in section 3.2 .2 that if self scattering of cosmic rays is the dominant mode by which cosmic rays are scattered in interstellar space, then the escape of cosmic rays from the Galaxy is energy dependent, resulting In a decrease of path length in the Disk with increasing energy. By taking into account the distribution of ionized and neutral matter perpendicular to the plane, Holmes (1974) showed that the energy dependence of path length is effective above a few $\mathrm{GeV} / \mathrm{n}$ and can be approximated as $\alpha \mathrm{E}^{-\alpha}$ above $30 \mathrm{GeV} / \mathrm{n}$, where $\alpha<0.38$. Many of the explanations so far put forward are based on this principle (Juliusson et al. 1972; Smith et al. 1973a; Audouze and Cesarsky, 1973; Meneguzzi, 1973a; Ramaty et al. 1973; Webber et al. 1973b). For steady state models, the equilibrium intensity of $i^{\text {th }}$ kind of nuclei can be written from Eqn. 3.27 as

$$
j_{i}(E)=\frac{q_{i}(E)+\sum_{k>i} P_{k i}(E) j_{k}(E)}{\left[1 / \Lambda_{i}+1 / x(E)\right]} .
$$

and hence, the ratio of secondary nuclei to primary nuclei $j_{S} / j_{P} \alpha X(E) /\left(X(E)+\Lambda_{S}\right)$ while that of two different primaries $j_{P_{1}} / j_{P_{2}} \alpha\left(X(E)+\Lambda_{2}\right) /\left(X(E)+\Lambda_{1}\right)$. From the observed change in composition, different workers obtained simple power law energy dependence for $X$ with a value of $\alpha$ varied from about 0.2 to 1.0 ( in / one can introduce higher order empirical relations like the one used by Cesarsky and Audouze, 1973). From Flgures 4.13 and basis we have shown 4.14, we find that the value of $\alpha$ is close to 0.2 ; and on this/in Figures 4.15 and 4.16 the calculated ratios of primary nuclei $(\mathrm{C}+0) /(\mathrm{Fe}$-group) and $(10 \leq Z \leq 14) /($ Fe-group) respective1y (Curve $A$ ) for $\alpha=0.2$. It can be seen from these figures that the observed variation of the ratios of 
primaries is more than that predicted from the variation of the ratios of secondary to primary nuclet. This has prompted Cesarsky and Audouze (1973) to postulate an energy dependent source composition and Ramaty, et al. (1973) to seek for an alternate source to account for the difference. The present experimental observations, with large systematic uncertainties, do not demand such hypothesizing at this stage.

The energy dependent confinement of cosmic rays in the galaxy lead to the following difficulties (Rengarajan, et al, 1973). Firstly, the very low life time implied in this model at high energles would lead to large anisotroples in contradiction to the observed near isotropy (Section 3.3). Secondly, since the energy dependent propagation requires that the observed spectrum is the steepened part of the equilibrium spectrum of nuclei, the spectrum at injection will have to be flatter than that observed; thereby raising the energy requirement from cosmic ray sources. Thirdly, this energy dependence cannot continue indefinitely but should cease at some energy $E_{c}$ corresponding to a lifetime of less than $\sim 10^{4}$ yrs., the time taken for cosmic rays to stream along a field line at the speed of light to reach the surface of the Disk. Consequently, the cosmic ray spectrum should regain its original shape above $E_{c}$ unless there is a fortuitous coiricidence of the right amount of steepening in the injection spectrum at $\mathrm{E}_{\mathrm{c}}$. In order to overcome these difficulties, it has been suggested that there could be energy dependent confinement of cosmic rays in some local regions in the Galaxy.

(ii) Energy dependent confinement of cosmic rays in special regions

If cosmic rays diffuse away from their sources in an energy dependent fashion, the matter traversed by them would also be so dependent provided 
most of it is traversed within the source, where the density of matter is expected to be very large. By suggesting that cosmic rays above 3 $\mathrm{GeV} / \mathrm{n}$ have an energy dependent confinement of the type with $\mathrm{X}_{\mathrm{S}}=21 / \mathrm{E} \mathrm{g} \mathrm{cm}^{-2}$ in the source regions, Meneguzzi (1973b) was able to reproduce closely the observed spectral shape of nucleonic components; by virtue of this efficient confinement of low energy cosmic rays, these sources are expected to be sources of gamma rays through neutral pion decay. On the other hand, Cowsik and Wilson (1973) have speculated that the effective matter traversed during diffusion in the source region varies as $x_{S} \approx 1.7 \exp (-E /$ 7.85) $\mathrm{g} \mathrm{cm}^{-2}$ with a subsequent traversal of about $1.6 \mathrm{~g} \mathrm{~cm}^{-2}$ of interstellar matter during their confinement in the Galaxy. While these models overcome the difficulties of the earlier ones, they suffer from the following difficulties. First, these models assume exponential path length distriinside the source region butions with allowed zero path lengths/as in the case of steady state models (which assumes uniform source distribution in an infinite diffusive medium) instead of using diffusion equations characterizing the diffusion of cosmic rays from a point source; in the latter case, there should be a minimum finite amount of matter traversed by cosmic rays inside the source leading to a large depletion of ultra-heavy elements. Secondly, the energy dependent diffusion in the source would lead to a steep steady state spectrum of cosmic rays inside the source region. In consequence, the resultant steep electron spectrum would give rise to synchrotron emission with a spectral index $>0.8$, which is in contradiction to the observed radio spectral indices of supernova remnants which is $<0.5$ below about $5 \mathrm{GHz}$ (MAlne and H111, 1969). 
Rengarajan, et al. (1971) suggested that most of the matter traversed by cosmlc rays is in the dense clouds, where the fraction of time spent by them is about 5 times larger than the fractional volume occupied by clouds in the Galaxy. They also introduced (Rengarajan, et al., 1973) an energy dependent streaming velocity for cosmic rays in the cloud and tried to explain the observed energy dependent charge composition. Similar to the source model, the dense clouds are expected to be strong sources of pionic gamma rays. The main difficulty of this model is that it is hard to achleve under steady-state conditions. a higher density of cosmic rays inside the clouds than in intercloud medium, from where they propagate into the clouds. However, one cannot rule out the possibility that the gas density in interstellar medium fluctuate and, as a result, cosmic rays and magnetic fields are compressed and later released through ampipolar diffusion as required by this model. 
4.5 Propagation of the electron component and its source characteristics

Cosmic ray electrons in interstellar space consist of two components:

(i) the directly accelerated electrons and (ii) the secondary electrons. The directly accelerated electrons are generally assumed to be negatively charged because of various reasons including the evidence for the negligible intensity of antiparticles in cosmic rays and the fact that the fraction of positrons observed at energies greater than a $\mathrm{GeV}$ seems to be consistent with that of secondary production. The secondary component would consist of positive and negative electrons and their relative abundances vary as a function of energy as seen below. In order to understand the propagation and source spectra of cosmic ray electrons, it is essential first to determine the characteristics of secondary electrons resulting from collisions of cosmic ray nuclei with interstellar gas.

\subsubsection{Secondary electrons in the Galaxy:}

The well known processes through which secondary electrons are produced in the Galaxy are (1) decay of short lived particles 1ike charged pions, (ii) decay of neutrons and radio nuclei through beta decay and (iii) knock-on of atomlc electrons by fast moving particles. We shall briefly indicate the methods of calculating the production spectrum of secondary electrons through the above processes.

(i) Decay of short lived particles: The most abundant of the created particles produced in interactions of high energy cosmic ray nuclei with matter, are pions. The charged pions decay to electrons through muons as

$$
\begin{aligned}
\pi^{+} \rightarrow & \mu^{ \pm}+v_{\mu} \\
& \downarrow \\
& \mathrm{e}^{ \pm}+v_{\mu}+v_{e}
\end{aligned}
$$


Since the decay life times of these particles are very small compared to the time scales involved in the propagation of cosmic rays, one can assume the decays to be instantaneous in cosmic scale. The production spectrum of pions has been derived by many authors in the past as reviewed by Danfel and Stephens (1970) and recently by Chocate and Wayland (1972) and Dooher (1973). However, it needs to be mentioned here that the results of these calculations are not in as good agreement with each other as one would like it to be mainly because the experimentally determined production spectrum of pions in the laboratory system, is not available at all energies of relevance here.

We will now start with the production of pions $Q_{\pi}(W) d W$, which is defined as the number of pions of total energy $W$ in the interval dW produced per gram of hydrogen in interstellar space per second, to derive the production spectrum of electrons; here $\pi$ denotes both positive and negative pions unless stated otherwise. It needs to be pointed out that the contribution of electrons from the decay of other short lived particles like K-mesons can be taken care of by sultably adjusting the parameters in $Q_{\pi}(W)$. The production spectrum of muons can then be written as

$$
Q_{\mu}(W) d W=\int_{W^{\prime}}^{W / f}=W+\varepsilon \quad Q\left(W^{\prime}\right) d W^{\prime} \frac{d W}{\psi_{\mu}(W)}
$$

Here $\varepsilon=m_{\pi}-m_{\mu}$ and $\psi_{\mu}(W)=W(1-f)$, where $f$ is the minimum fractional energy the muon gets from the pion and is given, by the relation

$$
\mathbf{f}=\frac{1}{2}\left[\left(1+\mathrm{m}_{\mu}^{2} / \mathrm{m}_{\pi}^{2}\right)-\left(1-\mathrm{m}_{\mu}^{2} / \mathrm{m}_{\pi}^{2}\right) \sqrt{1-\mathrm{m}_{\pi}^{2} / \mathrm{E}_{\pi}^{2}}\right] \quad 4.16
$$

which is equal to 0.56 for $\mathrm{W}_{\pi} \gg \mathrm{m}_{\pi^{*}}$. The production spectrum of electrons is then given as 


$$
Q_{e}(W) d W=\int_{W^{\prime}=\varepsilon}^{\infty} Q_{\mu}\left(W^{\prime}\right) d W^{\prime} \psi_{e}\left(W^{\prime}, W\right) d W \quad 4.17
$$

where $\varepsilon=W$ for $W>m_{\mu}$ and $\varepsilon=m_{\mu}$ when $W \leq m_{\mu} ; \psi_{e}\left(W^{\prime}, W\right) d W$ is the probability that, during the decay of muons of energy $W^{\prime}$, the electron gets an energy $W$ in $\mathrm{dW}$ and can be expressed as (Zatsepin and Kuzmin, 1962).

$$
\begin{aligned}
\psi_{\mathrm{e}}\left(\mathrm{W}_{\mu}, \mathrm{W}\right) \mathrm{dW}= & \frac{16}{(1-\beta)^{3}\left[3\left(1-\beta^{2}\right)-\frac{4}{3}\left(3+\beta^{2}\right) \mathrm{W} / \mathrm{W}_{\mu}\right]\left(\mathrm{W} / \mathrm{W}_{\mu}\right)^{2} \mathrm{dW} / \mathrm{W}_{\mu}} \\
& \quad \text { for } 0 \leq \mathrm{W} / \mathrm{W}_{\mu} \leq(1-\beta) / 2 \\
= & \frac{1}{\beta}\left\{\frac{5}{3}+\frac{4}{(1+\beta)^{3}}\left[\frac{8}{3} \mathrm{~W} / \mathrm{W}_{\mu}-3(1+\beta)\right]\left(\mathrm{W} / \mathrm{W}_{\mu}\right)^{2}\right\} \mathrm{dW} / \mathrm{W}_{\mu} \\
& \text { for }(1-\beta) / 2 \leq \mathrm{W} / \mathrm{W}_{\mu} \leq(1+\beta) / 2
\end{aligned}
$$

where $\beta$ is the velocity of muons relative to that of light.

(ii) Decay of neutrons and radio nuclei: Neutrons are produced mainly through charge exchange processes during proton collisions and the break up of helium nuclei, while radio nuclei result from the fragmentation and evaporation of incident and target nuclei respectively. The production spectrum of secondary neutrons and radio nuclei $Q_{s}(E) d E$ per gram of interstellar material per second can be written as

$$
Q_{s}(E) d E=4 \pi \underset{i, k}{\sum} d_{i} E_{i}\left(E_{i}\right) n_{k} \sigma_{s, i k}\left(E_{i}\right) \psi_{s}\left(E_{i}, E\right) d E
$$

where $E_{i}$ and $E$ are the kinetic energies of the incident and secondary nuclef respectively, $j_{1}$ is the flux of primary cosmic ray nuclei of the type $i$ in interstellar space, $n_{k}$ is the number of nuclei of the type $k$ per gram of interstellar gas, $\sigma_{s, i k}$ is the cross-section for the production of a particle of type $s$ and $\psi_{s}$ is the normalized energy distribution of the secondary particle during the collision. The cross section for neutron production and the mean energy $E^{\prime}=\int \psi_{S}\left(E_{i}, E\right) E d E$ as a function of $\mathrm{E}_{1}$ are summarized by Ramaty and Iingenfelter (1966a); the integral in 
Equation 4.19 can then be replaced by substituting $E_{1}=f E$, where $E$ is the mean energy:- Knowing the production spectrum of unstable nuclei, one can calculate the production spectrum of electrons using the relation (Ramaty, et al., 1970b)

$$
\mathrm{Q}_{\mathrm{e}}(\gamma) \mathrm{d} \gamma=\sum_{s} \frac{\mathrm{g}}{2} \mathrm{~d} \gamma \int_{1}^{\gamma_{\mathrm{m}}} \frac{\mathrm{d} \gamma^{*} \psi_{\mathrm{e}}\left(\gamma^{*}\right)}{\sqrt{\left(\gamma^{*}-1\right)}}{\underset{\gamma}{\gamma^{\prime}-}+\frac{\mathrm{d} \gamma^{\prime} \mathrm{Q}_{\mathrm{s}}(\gamma)}{\sqrt{\gamma^{\prime 2}-1}}}^{\gamma^{\prime}} \quad 4.20
$$

where $\gamma^{\prime}, \gamma, \gamma^{*}$ are the Lorentz factors of the unstable nuclei, electrons in the laboratory system and electrons in the rest system of the parent nuclei respectively, $\gamma_{\mathrm{m}}$ is the maximum energy of the electrons in the rest system of the parent nuclei and $\psi_{e}\left(\gamma^{*}\right)$ is the Fermi distribution function (Fermf, 1934):

$$
\psi_{e}\left(\gamma^{*}\right)=\gamma^{*}\left(\gamma^{*}-1\right)^{\frac{1}{2}}\left(\gamma_{m}-\gamma^{*}\right)^{2}
$$

The constant $g$ is the normalization factor for the Fermi distribution defined as $\mathrm{g}^{\gamma_{\mathrm{I}}} \psi\left(\gamma^{*}\right)=\mathrm{p}$, where $\mathrm{p}$ is the probability of electron decay through the given mode. The limits in Equation 4.20 are given as

$$
\gamma_{ \pm}^{\prime}=\gamma * \gamma \pm\left(\gamma^{* 2}-1\right)^{\frac{1}{2}}\left(\gamma^{2}-1\right)^{\frac{1}{2}}
$$

The lowest value of $\gamma^{\prime}$ is also determined by the minimum energy upto which the cosmic ray nucleon spectrum is assumed to exist. Some of the radio nuclei like $\mathrm{C}^{11}, \mathrm{~N}^{13}, 0^{14}$ and $0^{15}$ decay through positron mode and hence positrons are also produced in this process.

(iii) Knock-on process: In this process, energy is transferred to electrons of interstellar atoms in Coulomb collisions of cosmic ray nuclei. The cross section for knock-on production has been calculated by Bhabha (1938) and according to which, for spin $\frac{1}{2}$, we can write the differential probability, that a cosmic ray nucleus of charge $z_{j}$ and total energy per nucleon $W^{\prime}$, collides with an atom of charge $Z_{i}$ and 
atomic mass $A_{1} \mathbb{m}_{p}$ giving rise to knock on electrons of total energy $W$, as (Danie1 and Stephens, 1974)

$$
\begin{aligned}
& \phi_{i j}\left(W^{\prime}, W\right) d W=\frac{2 \pi N_{o} z_{i} r_{e}^{2} z_{j}^{2} m_{e}}{A_{i}\left(W^{\prime 2}-m_{p}^{2}\right)} \int \frac{W^{\prime 2}}{\left(W-m_{e}^{2}\right)^{2}}- \\
& \left.\frac{2 A_{i} m_{e} W^{p}+m_{e}^{2}+A_{i}^{2} m_{p}^{2}}{2 A_{i}^{2} m e\left(W-m_{e}\right)}+\frac{1}{2 A_{i}^{2}}\right) d W c^{2} g^{-1}
\end{aligned}
$$

where $N_{o}$ is Avogadro's number and $x_{e}$ is the classical radius of the electron. The production spectrum of knock on electron can be written as

$$
Q_{e}(W) d W=4 \pi \int_{W^{\prime}=\varepsilon}^{\infty} \sum_{i} \sum_{k} \phi_{i k}\left(W^{\prime}, W\right) d W j_{k}\left(W^{\prime}\right) d W^{\prime} \quad 4.24
$$

where $j_{k}\left(W^{\prime}\right)$ is the flux of cosmic ray nuclei of $k^{\text {th }}$ type. One can, for practical purposes, replace the sumation sign from the above equation by a factor of 1.82 to take care of the composition of interstellar gas and cosmic ray nuclei, and defining all the quantities in terms of protons.

We have shown in Figure 4.17, the differential production spectrum of secondary electrons per unit gram of hydrogen (after taking into account the composition of interstellar gas), per second through various processes described above. The curves in this figure have been obtained as follows: (a) the knock on spectrum (Curve A) is the modified version of the spectrum given by Abraham et a1. (1966); (b) the electrons from neutron decay (Curve B) is taken from Ramaty and Lengenfelter(1966a), (c) the pion decay spectra of both negative (Curve C) and positive (Curve D) electrons below a $\mathrm{GeV}$ have been evaluated from the work of Ramaty and Ligenfelter (1968) and Perola et al. (1967) and above $1 \mathrm{GeV}$ from Stephens (1969) by assuming a charge ratio $\mathrm{e}^{+/ \mathrm{e}^{-}} \approx 1.3$ same as that of the observed muons at high energies; and (d) the positron spectrum from radio nuclei is from the work 


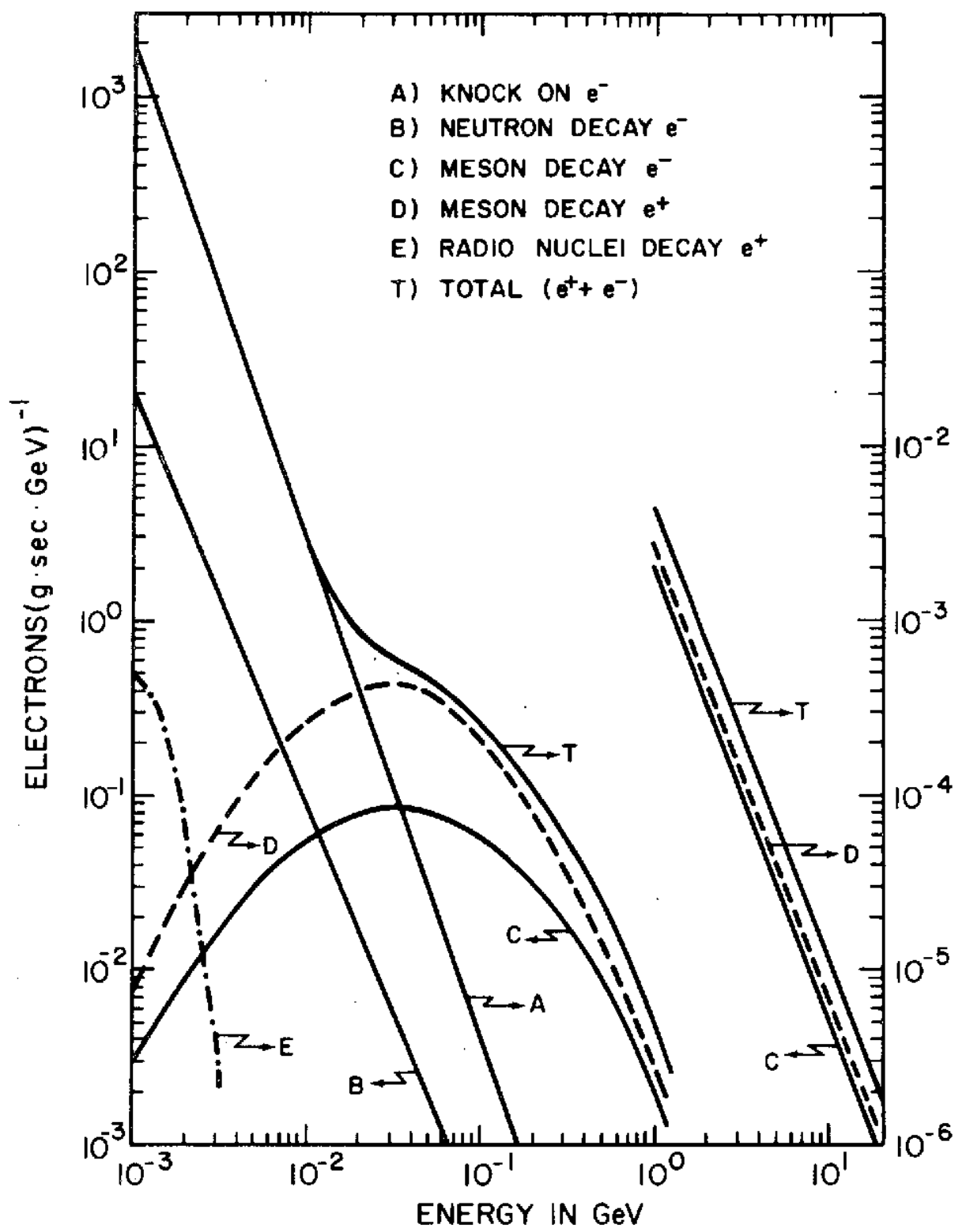

Fig. 4.17: Production spectra of secondary electrons in interstellar space through different processes. 
of Ramaty, et a1. (1970b) and Verma (1969). One can see from this figure that at energies below $30 \mathrm{MeV}$, the knock on spectrum dominates over other $e^{-}$spectra, while at energies above $50 \mathrm{MeV}$, electrons from pion decay dominate. Though the $\mathrm{e}^{-}$spectrum from radio nuclei is not included here, it can be easily shown from arguments similar to those given in (if) that their contribution would be much smaller than that from neutron decay at energies above a MeV. The positron spectrum is dominated by pion decay at all energies except below $2 \mathrm{MeV}$, where the decay of radio nuclei is important. The total secondary production spectrum is shown by curve $T$; It cannot be represented by a simple power law because of the many processes which contribute at different energies. However, the production spectrum below $10 \mathrm{MeV}$ can be represented by a power law of the type

$$
Q_{e}(E) d E=2 E^{-2.86} d E \text { electrons } g^{-1} \sec ^{-1}
$$

where $\mathrm{E}$ is expressed in $\mathrm{MeV}$; also the spectrum above a $\mathrm{GeV}$ can be represented by another power law of the type

$$
Q_{e}(E) d E=4.6 \times 10^{-3}, E^{-2.6} \mathrm{dE} \text { electrons } \mathrm{g}^{-1} \mathrm{sec}^{-1} \quad 4.25 \mathrm{~b}
$$

where $\mathrm{E}$ is expressed in $\mathrm{GeV}$.

When once the secondary electrons are produced, they lose energy through Ionfzation, bremsstrahlung, synchrotron radiation and inverse Compton scattering during their propagation in interstellar space; some of them will also diffuse out of the confinement volume of space. We shall now calculate the equilibrium spectrum of electrons under the known astrophysical conditions in the Galaxy by incorporating in addition a continuous acceleration in interstellar space. The equilibrium spectrum can be obtained by solving Equation 3.29; the values of the parameters $a, b$, $\alpha$ and $\tau$ in this equation can be estimated in the following manner. 
TABLE 4.3

Parameters used in calculating the equilibrium spectrum of electrons

\begin{tabular}{|c|c|c|c|c|c|c|}
\hline Region & $\begin{array}{c}\mathrm{N}_{\mathrm{H}} \\
\text { atoms } \mathrm{cm}^{-3}\end{array}$ & $\mathrm{GeV} \mathrm{sec}^{-1}$ & $\mathrm{GeV}^{-1} \mathrm{sec}^{-1}$ & $\begin{array}{c}\tau \\
\text { sec } \\
\end{array}$ & $\begin{array}{c}\alpha \\
\sec ^{-1}\end{array}$ & $\begin{array}{l}\text { in } \\
4.18 \\
\end{array}$ \\
\hline Disk & 1.0 & $2.22 \times 10^{-16}$ & $1.75 \times 10^{-16}$ & $6 \times 10^{13}$ & $<<1 / \tau$ & $\mathbf{A}$ \\
\hline$"$ & " & " & $7.75 \times 10^{-16}$ & "1 & " & B \\
\hline$"$ & $n$ & " & " & $"$ & $1 / \tau=1-67 \times 10^{-14}$ & C \\
\hline Halo & $1.0 \times 10^{-2}$ & $2.22 \times 10^{-18}$ & $7.5 \times 10^{-17}$ & $6 \times 10^{15}$ & $<<1 / \tau$ & D \\
\hline$"$ & " & " & " & " & $1 / \tau=1.67 \times 10^{-16}$ & E \\
\hline
\end{tabular}


(1) Since ionization is of Importance only below about $20 \mathrm{MeV}$,

$a \approx 2.22 \times 10^{-16} n_{H}$ GeV sec, where $n_{H}$ is the mean number of hydrogen atom $\mathrm{cm}^{-3}$ in interstellar space. (ii) The value of $\mathrm{b}$ can be written as $b \approx 10^{-16} \times\left(3.8 \times 10^{-2} \mathrm{~B}_{\perp}^{2}+\rho_{t}\right) \mathrm{GeV}^{-1} \mathrm{sec}^{-1}$, where $\mathrm{B}_{\perp}$ is the mean perpendicular component of the magnetic field in $\mu G$ and $\rho_{t}$ the total photon density in $\mathrm{eV}$ in the Galaxy. (i1i) The residence time $\tau$ is taken to be about $2 \times 10^{6}$ yrs for the mean matter traversal of about $3 \mathrm{~g} \mathrm{~cm}^{-2}$ of hydrogen by cosmic rays. (iv) The value of the acceleration parameter $\alpha$ is kept as a free parameter. Table 4.3 sumarizes the parameters used in evaluating the equilibrium spectrum of secondary electrons. It needs to be mentioned here that in the case of the Disk the existence of the submillimeter radiation has not been taken into account in calculating the value of $b$ for Curve $A$ while for Curve $B$ it has been included; for the Halo its existence has been neglected.

In Figure 4.18 the equilibrium spectra of electrons are shown for varlous set of parameters summarized in Table 4.3. In the absence of acceleration, the spectral shape of the equilibrium electrons remains as the production spectrum (Figure 4.17)

the same/in the energy region between about $20 \mathrm{MeV}$ and a few GeV (Curves $A, B$ and D). Below $20 \mathrm{MeV}$, the spectrum is flatter due to fonization loss (Equation 3.32a)/above a few GeV, the spectrum steepens due to synchrotron and inverse Compton processes (Equation 3.32c); the energy at which this steepening occurs is inversely proportional to the product of the residence time and the parameter $b$. It can also be seen from Curve A that continuous acceleration is not at all effective for values of $\alpha \leq \sqrt{4 \mathrm{ab}}$. For $\alpha>\sqrt{4 \mathrm{ab}}$, it is only marginal if $\alpha \ll 1 / \tau$ even in the energy region $\alpha-\sqrt{\alpha^{2}-4 a b}<2 b E<\alpha+\sqrt{\alpha^{2}-4 a b}$, where acceleration 


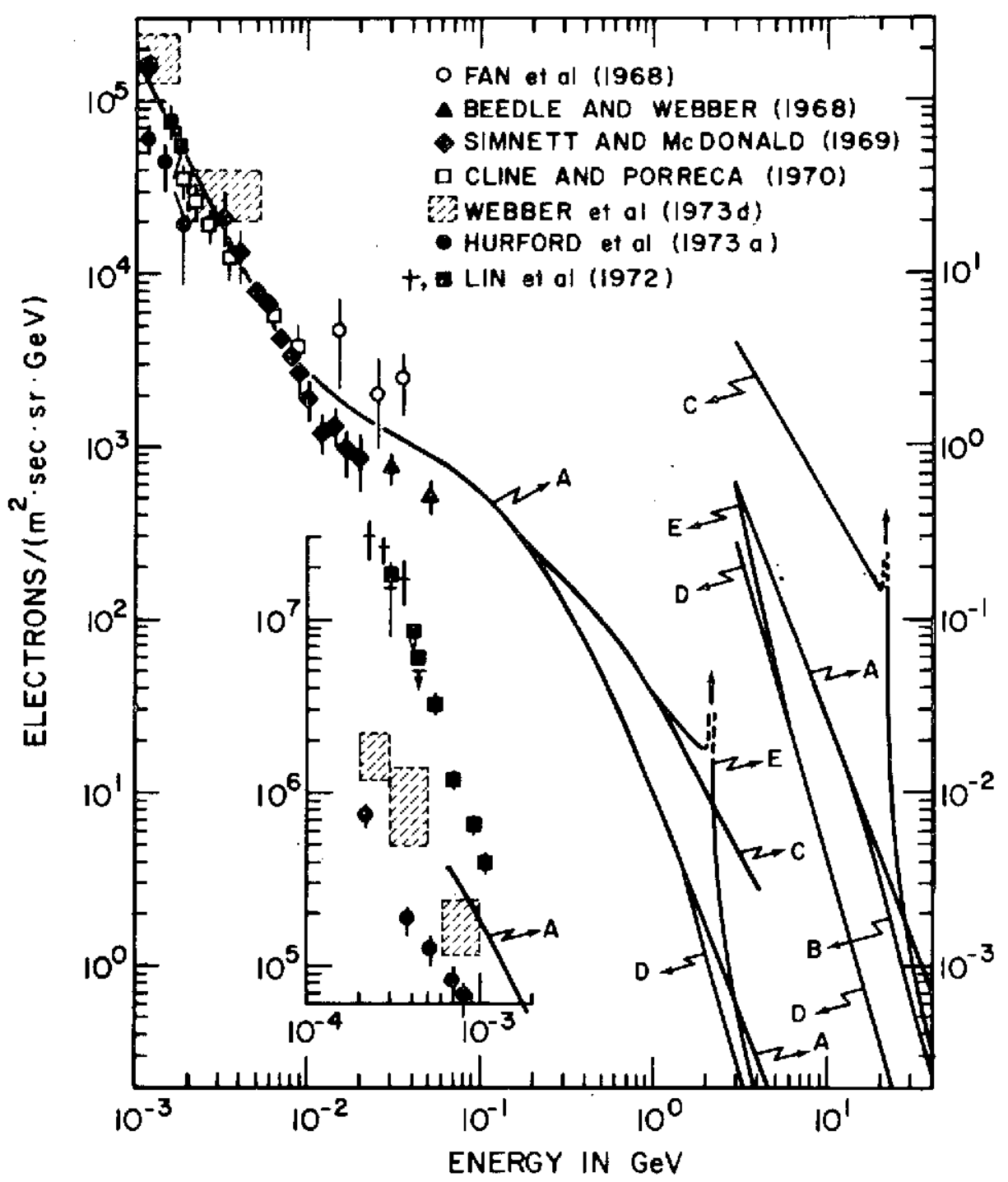

Fig. 4.18: Equilibrium spectra of secondary electrons in the Galaxy for various interstellar parameters summarized in Table 4.4; the observed intensities below a few tens of MeV are also shown. 
dominates over continuous energy loss processes. However, the acceleration becomes important as the value of $\alpha$ approaches $1 / \tau$ and when $\alpha=1 / \tau$, the equilibrium condition is never reached at energy $E_{S}=\left(\alpha+\sqrt{\alpha^{2}-4 a b}\right) / 2 b$ and the flux increases as the ratio of the age of the confinement volume of space to $\tau$. These calculations clearly demonstrate that if interstellar acceleration is effective, the flux of secondary electrons is very much enhanced in the region above a few hundred MeV. However, observations on positrons do not indicate any such trend and hence it may be stated that continuous acceleration in interstellar space is either absent or ineffective.

\subsubsection{Directly accelerated electrons}

In order to deduce information on the directly accelerated electrons, it is essential to know first the equilibrium spectrum of the total cosmic ray electrons in interstellar space. Thereafter, by subtracting the contribution from secondary electrons, one can derive the intensity and spectral shape of directly accelerated electrons under equilibrium conditions (Daniel and Stephens, 1970a). The energy spectrum of the electron component in interstellar space can be obtained by different methods: (i) One can demodulate the observed electron spectrum by making use of the modulation parameters, deduced from an analysis of the temporal variation of the nucleonic and electronic components and a study of the propagation of nucleonic component; (11) It can be deduced from the galactic radio background; and (iii) It can also be inferred from a comparison of the observed positrons and the expected secondary positrons in interstellar space.

In Figure 4.19, Curve $A$ is the interstellar radio emitting electron spectrum as deduced in Section 5.4 .2 by using method (ii) and Curve $B$ is the 


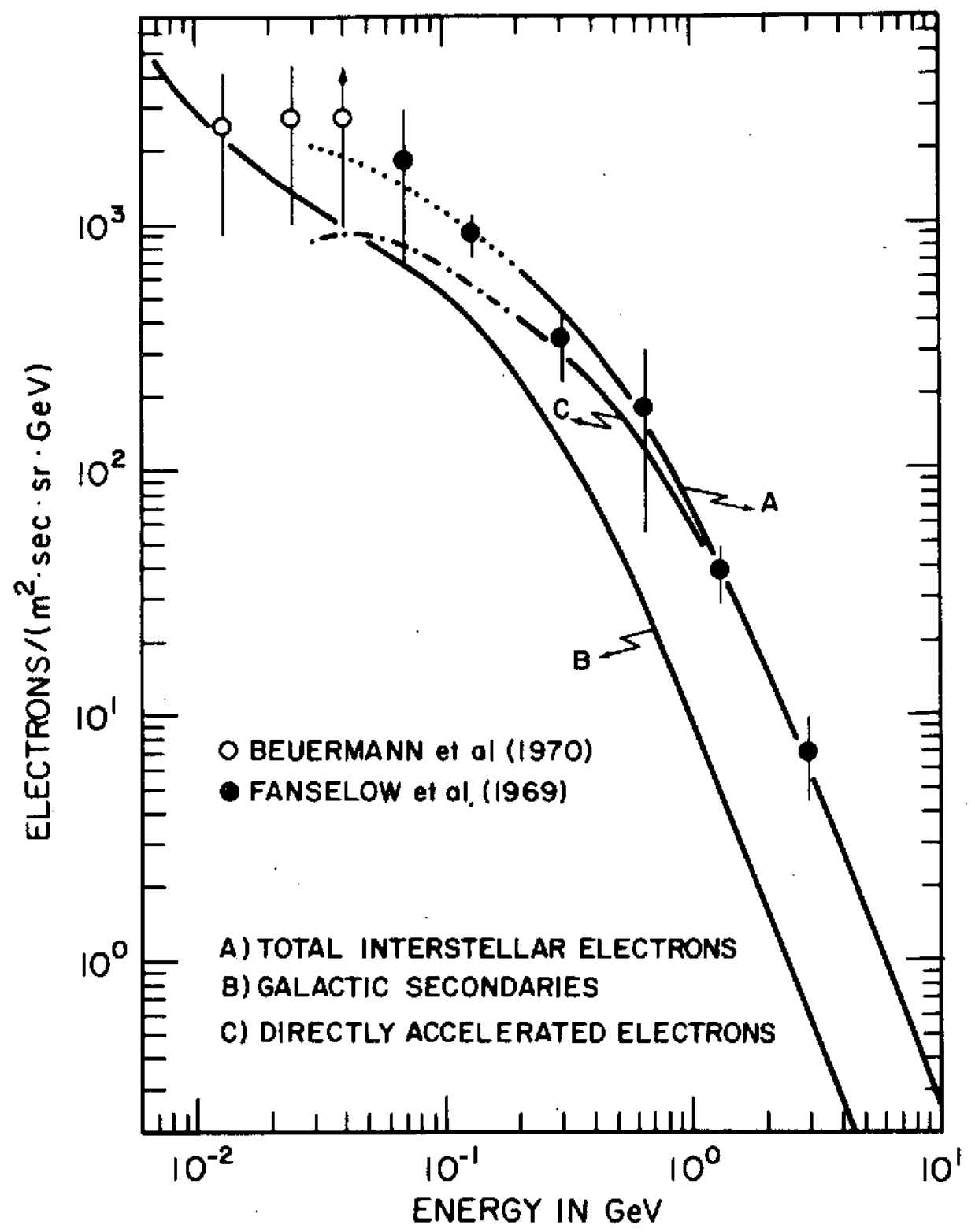

Fig. 4.19: The radio emitting electron spectrum in the Galaxy (Curve A) is compared with that deduced from the observed positrons (data points); Curves $B$ and $C$ are the equilibrium spectra of secondary and directly accelerated electrons respectively. 
calculated equilibrium spectrum of secondary electrons in interstellar space. The points in Figure 4.19 are the calculated intensities $j_{e}(E)$ of electrons in interstellar space using the observed fraction of positrons $f_{+}(E)$ as a function of energy (Beuermann, et al., 1970; Fanselow, et al., 1969) and the relation $j_{e}(E)=j_{+}(E) / f_{+}(E)$, where $j_{+}(E)$ is the estimated secondary positron flux in the Galaxy. It is seen from this figure that at energies $>200 \mathrm{MeV}$, the total electron spectrum deduced from method (ii) is in good agreement with that obtained by method (iii). Hence, it is reasonable to extend Curve A below $200 \mathrm{MeV}$ on the basis of the observed positron fraction; this is shown by the dotted curve in Figure 4.19 .

Having deduced the equilibrium spectrum of total electrons in interstellar space, the energy spectrum of directly accelerated electrons can be obtained by subtracting from the above the calculated spectrum of secondary electrons. This method, first employed by Daniel and Stephens (1970a), demonstrated that the directly accelerated electron spectrum flattens gradually below $2 \mathrm{GeV}$ such that the flux probably becomes insignificant at very low energles; the revised spectrum as obtained from the present data is shown as Curve $C$ in Figure 4.19. This spectrum is found to dominate over that of secondary electrons (Curve B) above $2 \mathrm{GeV}$ by a factor of about 10 , having a spectral index $\beta \simeq 2.6$ as required by the radio spectra (section 5.4) and is similar to those of nucleonic components. Below this energy the contribution of secondary electrons slowly increases and perhaps overwhelming below about a few tens of $\mathrm{MeV}$, where the calculated secondary electrons of Figure 418 accounts fully for the observed flux; a similar conclusion was drawn recently by Cummings; et al. (1973a). 


\subsubsection{The origin of cosmic ray electrons}

The relatively low abundance of electrons compared to protons in cosmic rays demands an explanation as to why charge neutrality, which is universal in all natural phenomena, is not preserved in cosmic radiation. However, a satisfactory explanation to this most intriguing problem is still to be proposed in the future; therefore in what follows in this section, we shall briefly outline our present knowledge on the origin of cosmic ray electrons in a number of suitable energy intervals.

(1) $\mathrm{e}^{ \pm}$below $1 \mathrm{MeV}$ : The existence of cosmic ray electrons with energies $\mathrm{E}<1 \mathrm{MeV}$ in interplanetary space was first detected by Beedle, et al., (1970), who showed that the flux of electrons in the region of a few hundred keV seems to fall close to the extension of the observed spectrum above a few MeV. Recently these measurements have been extended down to energies about a few tens of keV (Lin, et al., 1972; Webber, et al., 1973d; Hurford, et al., 1973a) and from the observed temporal variations, electrons above $100 \mathrm{keV}$ are considered to be of galactic origin. One notices from the inset of Figure 4.18 that at energies $<1 \mathrm{MeV}$, the flux values of Lin, et al., are about an order of magnitude larger than those of Hurford, et a1.; this large steady flux of Lin, et al., could be due, as pointed out by Hurford, et al., to a substantial spacecraft background and long-term averaging. Since the electron spectrum of Hurford, et al., and Webber, et a1., are consistent with the extension of galactic knock-on spectrum (Figure 4.18), one is inclined to consider these low energy electrons to be of secondary origin; the present situation therefore doesn't warrant any new mechanism to be invoked (Ramaty, et a1., 1972) to understand the observations. 
(i1) $e^{+}$around $1 \mathrm{MeV}$ : In the energy region between 0.5 and $2 \mathrm{MeV}$, an anomalous finite flux of positrons was reported by c1ine and Hones (1970), while the observed positron intensity above $3 \mathrm{MeV}$ is down by a factor of about $10^{2}$ (Cline and Porreca, 1970). In Fig. 4.20, we show the observed intensities of positrons from about $200 \mathrm{keV}$ to $20 \mathrm{MeV}$ (Cline and Hones, 1970; Cline and Porreca, 1970; Beuermann, et al., 1970; Hurford, et al., 1973b) along with theoretical estimates. Though recent measurements of Hurford, et a1., provide on $1 y$ upper limits, the suggestion of a sharp decrease of the intensity between 2 and $10 \mathrm{MeV}$ indicate that perhaps these positrons arise from the decay of radio nuclei. Estimates have been made of the positron spectrum from radio nuclei, produced during the collision of cosmic ray nuclei with interstellar gas for various assumed primary spectra (Verma, 1969; Ramaty, et al., 1970b). Curve A in Figure.4.20 is a calculated spectrum by Ramaty, et al., for a power law spectrum of cosmic rays in kinetic energy to exist in interstellar space up to an energy $E_{c}=$ $5 \mathrm{MeV} / \mathrm{n}$. This explanation, however, requires a cosmic ray energy denstty of $\sim 100 \mathrm{eV} / \mathrm{cm}^{3}$ in interstellar space, and a modulation factor $\sim 10$ to be operative for positrons only up to an energy just above $2 \mathrm{MeV}$.

An alternate explanation was put forward by Burger, et al. (1970) and Colgate (1970), who suggested that these positrons could arise from the decay of ${ }^{56} \mathrm{Co} \rightarrow{ }^{56} \mathrm{Fe}$ in the silicon burning shells of supernovae just after their ejection at relativistic energies.Curve $B$ in Figure 4.20 is the calculated equilibrium spectrum of positrons by Burger, et al., using a power law spectrum in rigidity for the ${ }^{56} \mathrm{~N} 1$, which first decays to ${ }^{56} \mathrm{Co}$, in the source region with $R_{C}=30 \mathrm{MV}$; Curve $C$ is for $R_{c}=100 \mathrm{MV}$ when ionization 


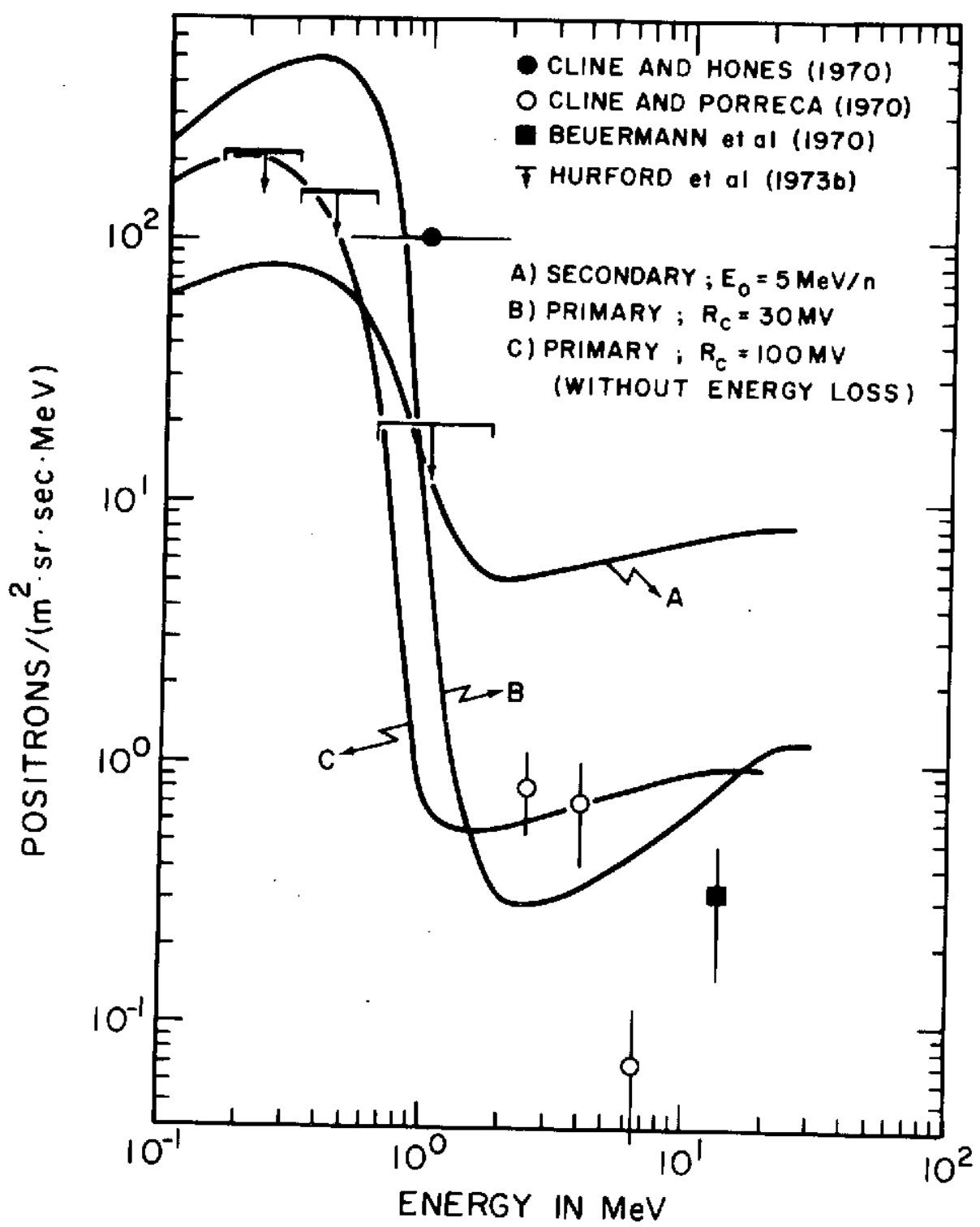

Fig. 4.20: The observed positron spectrum is compared with the theoretical spectra based on their production in interstellar gas (Curve $A$ ) and in supernova envelopes (Curves $B$ and $C$ ). 
loss is not taken into account. This explanation requires that most of the iron nuclei in cosmic rays result from the decay of ${ }^{56} \mathrm{Ni}$, which are produced during efficient burning of silicon in the supernova shells (Bodansky, et al., 1968). Subject to the reality of the above situation, this hypothesis is more attractive than the earlier one (Curve A) both from the point of view of better agreement with observations and more plausible assumptions regarding the primary spectrum.

(iii) $\mathrm{e}^{ \pm}$between 1 and $10 \mathrm{MeV}$ : The observed intensity and charge composition of electrons in this energy region can be explained as due to the combination of interstellar knock-on and pion decay electrons (FIgure 4.18). It needs to be pointed out at this stage that it is assumed here that at energies < $10 \mathrm{MeV}$ the observed intensity truly represents the interstellar spectrum because of the absence of any detectable long-term variation of their intensity (Simnett and McDonald, 1969). (iv) $\mathrm{e}^{ \pm}$between 10 and $100 \mathrm{MeV}$ : In this energy region, the electrons undergo modulation and one is unable to fully account for the intensity on the basis of the secondary hypothesis. Further, the observed charge composition in this energy region suggests that a comparable fraction of electrons in this energy range is directly accelerated (Figure 4.19). (v) $\mathrm{e}^{ \pm}$of energy $>100 \mathrm{MeV}$ : Electrons in this energy domain are very Important since directly accelerated electrons influence the spectral shape and account for about $90 \%$ of the total electrons above a few $\mathrm{GeV}$. One would therefore like to examine whether the spectral shape of directly accelerated electrons in equilibrium is representative of the injection spectrum or is modified due to energy loss processes. Many arguments have 
been put forward in the past, as summarized by Daniel and Stephens (1970), that the gradual steepening of the electron spectrum between a few hundred $\mathrm{MeV}$ and a few $\mathrm{GeV}$ is not due to energy loss processes but intrinsic to the injection spectrum. Further, one notices from Figure 4.19 that the spectral shape of directly accelerated electrons gradually changes by a power index $|\Delta \beta| \geqslant 1.5$, which cannot be understood on the basis of energy loss processes in interstellar space. In this regard an accurate determination of the positron spectrum experimentally is very valuable because one can compare this with the production spectrum to look for any propagation effects. Notwithstanding this, the study of the electron spectrum above a few GeV plays an important role in the understanding of the propagation of cosmic rays and we shall discuss this aspect in some detall.

\subsubsection{Interpretation of high energy electron spectrum}

The available data on high energy electron spectrum above a few GeV, published after 1970 are plotted interms of energy flux units for convenience in Figure 4.21(Hovestadt, et al., 1971; Marsden, et al., 1971; Scheepmaker and Tanaka, 1971; Swanenburg, et al., 1971; Zatsepin, 1971; Earl, et al., 1972; Anand, et a1., 1973a; Burger and Swanenburg, 1973; Muller and Meyer, 1973; Meegan and Earl, 1973; Silverberg, et al., 1973; Ishii, et al., 1973; Webber and Rockstroh, 1973): the earlier results have previously been summarized by Daniel and Stephens (1970), Yash Pal (1969) and Meyer (1971). One notices from this figure that the spread of the data points due to various experimenters is disproportionally large, compared to what is expected on the basis of statistical errors attributed to the individual values, thereby clearly demonstrating the existence of large 


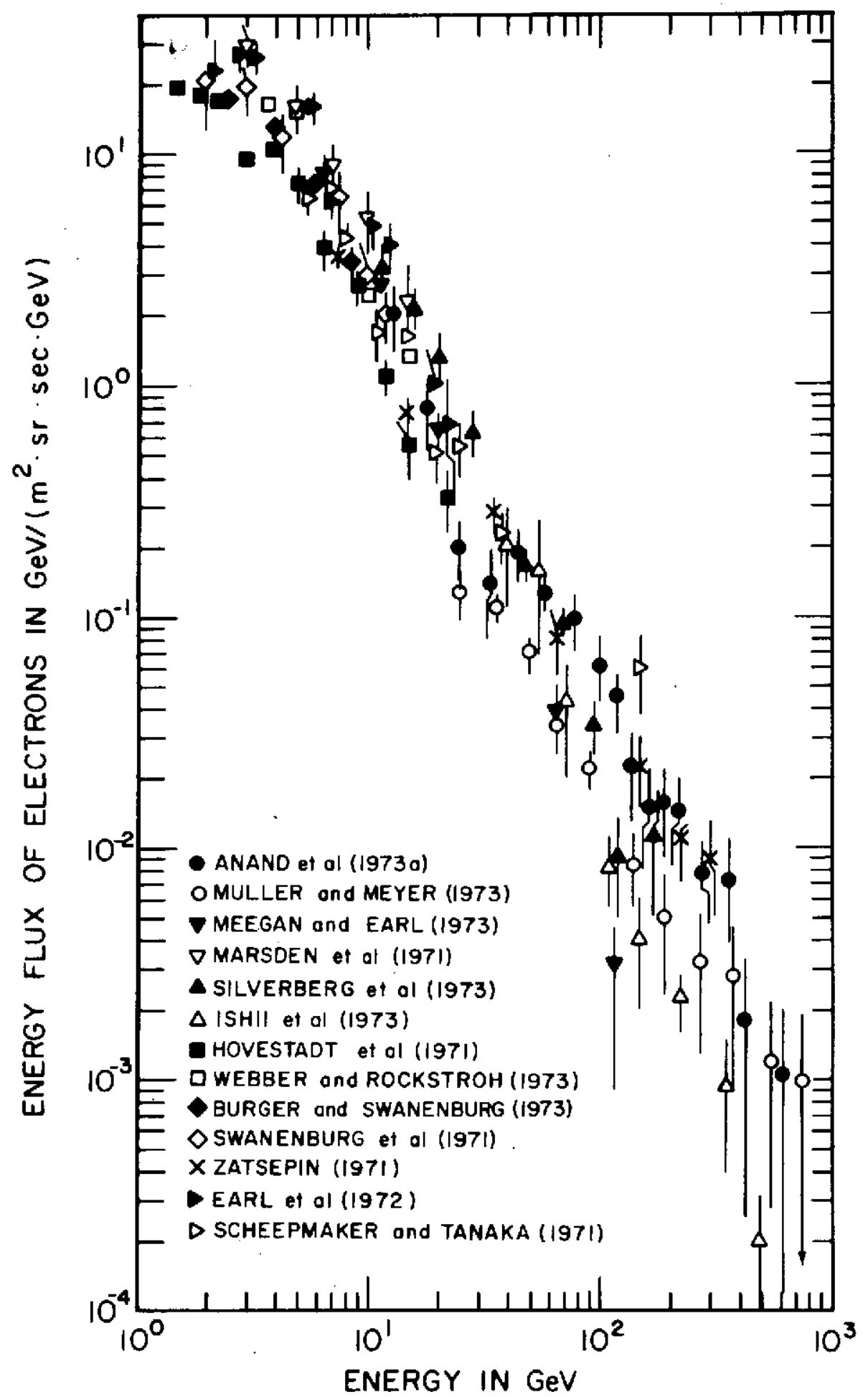

Fig. 4.21: The observed energy spectrum of cosmic ray electrons of energy greater than a few GeV. 
TABLE 4.4

Observed Differential Spectral Index of High Energy Electrons

\begin{tabular}{lcc}
\multicolumn{1}{c}{ Authors } & $\begin{array}{c}\text { Energy range } \\
\text { in GeV }\end{array}$ & $\begin{array}{c}\text { Spectral Index } \\
B\end{array}$ \\
\hline Swanenberg et al. (1971) & $3-10$ & $2.6 \pm 0.1$ \\
Marsden et al. (1971) & $3-15$ & $2.6 \pm 0.1$ \\
Earl, et al. (1972) & $5-25$ & $3.3 \pm 0.1$ \\
Webber and Rockstroh (1973) & $3-20$ & $3.0 \pm 0.2$ \\
Scheepmaker and Tanaka (1971) & $5-300$ & $2.7 \pm 0.1$ \\
Zatsepin (1971) & $5-600$ & $2.7 \pm 0.1$ \\
MuIler and Meyer (1973) & $30-1000$ & $2.7 \pm 0.1$ \\
Anand et al. (1973a) & $10-800$ & $2.7 \pm 0.1$ \\
" & $>150$ & $3.1 \pm 0.3$ \\
Ishii et al. (1973) & $>100$ & $3.2 \pm 0.2$ \\
Meegan and Earl (1973) & $10-100$ & $3.5 \pm 0.1$ \\
Silverberg et al. (1973) & $10-200$ & $3.2 \pm 0.2$ \\
\hline
\end{tabular}


systematic errors between them. This is again brought out in Table 4.4, where we have summarized the spectral indices due to the same authors. It 1s, therefore, neither correct nor possible to look for any steepening in the spectral shape from a plot of "World data." However, one can still make the following statements about the electron spectrum from Figure 4.21 and Table 4.4: (a) the cosmic ray electron spectrum extends at least up to about $700 \mathrm{GeV}$ with non-trivial flux values; and (b) there is no indication of a detectable change in the spectral index up to $100-200 \mathrm{GeV}$. We shall, therefore, attempt now to bring out the possible implications of the above observations on the basis of the theoretical models described in section 3.5 .2 .

(i) Single source mode1: In the case of cosmic ray electrons originating from a single source, one can notice: frow Equation 3.34 that electrons cannot reach the point of observation with energies $E \geq 1 / b\left(t-t_{0}\right)$. Since we observe a non-trivial flux of cosmic ray electrons at least up to $700 \mathrm{GeV}$, $\left(t-t_{0}\right)<3 \times 10^{5}$ yrs for $b \sim 5 \times 10^{9} \mathrm{GeV}^{-1} \sec ^{-1}$ (Table 4.3); a similar result has been arrived at recently from an analysis of the composition of UH nuclei (Kaiser, et a1., 1972). From the distribution of non-thermal radiation in the Galaxy, we infer (Section 5.4.4) that the intensity of cosmic rays in the neighborhood of the Sun is nearly the same as that elsewhere in the Galaxy and in order to achieve this, electrons should have enough time to diffuse to the point of observation from the source region. Thus, according to Equation 3.34, $4 D\left(t-t_{0}\right)>\left|\underline{r}-\underline{r}_{0}\right|^{2}$ and taking Vela $X$ to be the nearest plausible source of cosmic ray electrons (Shen, 1970), one can set a lower bound of $4 \times 10^{28} \mathrm{~cm}^{2} \mathrm{sec}^{-1}$ for the diffusion coefficient $D$. 
From the above it is quite clear that if a single source contributes to the observed cosmic ray electrons, then the mean free path for cosmic rays for isotropic diffusion is $\lambda>1$ pc which in turn leads to large anistropy. (ii) Flat disk model: Let us now consider the flat disk model in which sources are unfformly distributed and cosmic rays are in a state of equilibrium. In this case, one expects from Equations 3:36 and 3.37 two spectral steepenings, each with $|\Delta \beta|=0.5$ at energies $E_{1}$ and $E_{2}$ separated by a factor $E_{2} / E_{1} \sim R^{2} / L^{2}$. It has already been mentioned in Section 4.5 .3 that the observed electron spectrum shows a gradual steepening at about $2 \mathrm{GeV}$ and in spite of the fact that this spectral shape could be inherent to the direct1y accelerated electrons, one can still examine the consequences by ascribing this steepening to result from propagation effects under two possibilities. In the first, it may be said that this is the first spectral break corresponding to energy $\mathrm{E}_{1}$ and from the observed lack of any detectable spectral change at least up to $\sim 200 \mathrm{GeV}$, one could say that $\mathrm{R}^{2} / \mathrm{H}^{2} \geq 100$. If one sets $2 \mathrm{H} \sim 350 \mathrm{pc}$, the effective thickness of the gas disk, then the sources in the Disk which contribute to the observed radiation have to lie within a radius $\mathrm{R} \geqslant 1.75 \mathrm{kpc}$ from the Sun. Further, from Equation 3.36 one gets a lower 1imit to the diffusion coefficient $D \approx E_{1} \mathrm{bR}^{2} / 4 \geq$ $2 \times 10^{27} \mathrm{~cm}^{2} \mathrm{sec}^{-1}$ and a corresponding residence time $\tau \sim \mathrm{H}^{2} / 4 \mathrm{D} \leqslant 10^{6}$ yrs. Though these values seem attractive, there are a number of objections to be suitably explained. If, for example, the observed spectral index above a few $\mathrm{GeV}$ is close to 2.6 (Table 4.4), it means that the injection spectrum Is significantly flatter than that of cosmic ray nuclei carrying with it other serfous implications (Daniel and Stephens, 1970). On the other hand, 
If it is claimed that the spectral index is close to 3.1 (Table 4.4), then one runs into the following difficulties: ( 1 ) the radio spectral index should gradually steepen to a value of $(\beta-1) / 2 \approx 1.05$ beyond about a few hundred MHz; the available data summarized in section 5.3 .1 shows that the radio spectral index has a value of 0.8 from about $178 \mathrm{MHz}$ to a few $\mathrm{GHz}$. (ii) The positron spectrum should also have a spectral index of 3.1 beyond $2 \mathrm{GeV}$; Indications from the presently available observations (Fanselow, et a1., 1969) are not consistent with this, though better measurements are needed for a final decision in the future. Finally, this explanation of a break at $\imath_{2} \mathrm{GeV}$ also requires a second break of $|\Delta \beta|=0.5$ to be established beyond a few hundred GeV.

If we consider the other alternative that the apparent spectral change around $2 \mathrm{GeV}$ is due to the second break at $E_{2}$, then from Equation 3.36 we have $D \approx 2 \times 10^{25} \mathrm{~cm} \sec ^{-1}$ and $\tau \sim 10^{8} \mathrm{yrs}$. These values are not compatible with our knowledge of the propagation of cosmic rays in the Disk. Further, one requires that (i) the sources which contribute to the observed cosmic rays are distributed uniformly within a radius of about $175 \mathrm{pc}$, and (ii) the energy spectrum of electrons at injection should have a spectral index $\leqslant 2.0$, which is very different from that of cosmic ray nuclei, and (iii) the positron spectral index has to be $\approx 3.6$ in contradiction to the observation. Thus, we find that this explanation is less plausible than the previous one. Perhaps before concluding, mention may also be made of a third but less attractive possibility that even the first break has not occurred at least up to $200 \mathrm{GeV}$. 
(iii) Leakage lifetime model: On the basis of this model, the residence time of cosmic rays is about $10^{8}$ yrs and $\leqslant 10^{6}$ yrs corresponding to the possible breaks by one power around 2 and $200 \mathrm{GeV}$, respectively. However, arguments mobilized in the previous paragraphs in thts section would favor the second possibility. Furthermore, it is also possible to introduce an energy dependent leakage lifetime for electrons as in the case of nucleonic components (Silverberg and Ramaty, 1973). Accordingly, if one assumes a dependence of the type $\tau$ a $\mathrm{E}^{0.3}$ beyond about $5 \mathrm{GeV}$ (Curve $\mathrm{B}$ in Figure 4.13), one gets for the lifetime of cosmic rays in the $\mathrm{GeV}$ region a value $\leqslant 3 \times 10^{6}$ yrs on the basis that the electron spectrum doesn't show a finite steepening up to $200 \mathrm{GeV}$; some of the difficulties of this model have been summarized in section 4.4.3.

Thus, we find that there are many ways of interpreting the observed data on high energy electrons. It is evident that, though in principle some of the alternatives could be ruled out, it requires for this purpose more accurate and reliable observations on the spectra of both positrons and negatrons. In spite of the existing uncertainties, the overall evidence is in favor of a cosmic ray residence time $\sim 10^{6} \mathrm{yrs}$. It is also interesting to mention here that, since electron energy loss through synchrotron radiation is maximum when its pitch angle is perpendicular to the magnetic fleld lines, the high energy electrons in the local interste1lar space would be anisotropic with bi-directional maxima defining the local direction of the large scale magnetic field (Ear1 and Lenchek, 1969). 


\subsection{Leakage Life Time of Cosmic Rays}

The leakage life time (also differently referred to as dwell time, residence time, confinement time and storage time) of cosmic rays is defined as the mean amount of time for which the cosmic rays reside within the confinement volume of space; it'is characterised by the nature of the confinement volume, the distribution of sources within this volume of space and the diffusion mechanism. When diffusion is rapid and the storage volume has a reflecting boundary which inhibits free leakage, the mean age of cosmic rays sampled at any place within the confinement volume will be the same and will represent the residence time; also it will not depend upon the distribution of sources around the observer, except in case of a close proximity of a source or sources to the observer both in time and space. On the contrary, when diffusion is slow, the residence time is a meaningless concept because the mean life time of cosmic rays sampled by the observer is influenced more by the energy loss associated with the type of particle sampled and the distribution of sources around the point of observation, than by the nature of the confinement volume of space. In this section, we shall make use of the concept of leakage life time to understand the confinement volume of space by attributing the mean age of cosmic rays sampled near the Earth to be identical with the residence time of cosmic rays.

One powerful method of deducing information on the leakage life time is to take advantage of the sensitive dependence of the energy spectrum of cosmic ray electrons - among other factors - the residence time of cosmic rays. However, since this aspect is adequately covered in Sections 3.5 .2 (theoretical aspects) and 4.5 .4 (interpretative aspects) we will not go 
into $1 t$ here. One other 1mportant aspect of leakage life time which we have avolded mentioning in this section for a similar reason is the following. The interesting observations recently made from a study of the energy spectra of "primary" and "secondary" cosmlc ray nuclei in the energy range 5-100 $\mathrm{GeV} / \mathrm{n}$, and the many theories proposed to understand these observations on the basis of an energy dependent confinement have been described in detail in Section 4.4 .

\subsubsection{Mean Matter Traversed by Cosmic Rays and the Res1dence Time.}

It is a normal practice to relate, $\langle x\rangle$ the mean amount of matter traversed by cosmic rays, to the mean age of cosmic rays. Consequently, one can write $\tau=\langle x\rangle / \rho c$, where $\rho$ is the mean matter density in the confinement volume. It has been shown in Section 4.1 , that presently available observations suggest that $\langle x\rangle \approx 3-6 \mathrm{~g} \cdot \mathrm{cm}^{-2}$ of hydrogen in the $\mathrm{GeV}$ region. Using this value for $\langle\mathrm{x}\rangle$, one gets $\tau \approx(2-4) \times 10^{6}$ yrs for the Disk model where $\rho=1.67 \times 10^{-24} \mathrm{~g} \cdot \mathrm{cm}^{-3}$ of hydrogen while for the Halo model one gets $\tau \neq(2-4) \times 10^{8} \mathrm{yrs}$, where $\rho$ \& $1.67 \times 10^{-26} \mathrm{~g} . \mathrm{cm}^{-3}$ of hydrogen. These estimates of the leakage life time are justified under the assumption that the matter traversed by cosmic rays is in interstellar space and that matter is uniformly distributed over the entire volume of the storage space. However, it has been shown in Section 2.3 that about $70 \%$ of the interstellar gas is in the form of dense clouds. Even so, if cosmic rays spend equal amounts of time in equal volumes of space, the above estimate of $\tau$ will not be affected by the exact nature of the interstellar gas. Notwithstanding this, it has been found that magnetic fields in the clouds are high and are effectively detached from the intercloud medium. If these were so, one would expect cosmic rays 
to be confined inside clouds for a longer time than in a corresponding volume of intercloud medium; this situation would then tend to reduce the value of $\tau$. In a similar manner, if part of the matter is traversed In the source region, one would over-estimate the value of $\tau$ by attributing the entire matter traversed to be in interstellar space. Thus, in either case, the values of $\tau$ deduced above are likely to be upper 1imits. It needs to be emphasized at this stage that in this method $\tau$ can be estimated only by assuming a model for the cosmic ray confinement. 4.6.2 Leakage Life Time From Long Lived Radio Nuclei in Cosmic Rays

A direct method of estimating the mean age of cosmic rays is to determine, in the primary radiation, the abundance of a suitable long lived radio nucleus produced in secondary collisions. It has been suggested by Hayakawa et al (1958) and Peters (1963) that Be ${ }^{10}$, which decays to $B^{10}$ with a mean 1 ife of $1.6 \times 10^{6} \mathrm{yrs}$, could be used as a clock to measure the mean age of cosmic rays. The first attempt towards this was made by Daniel and Durgaprasad (1966) and later by many others, by making use of the ratio $\mathrm{Be} / \mathrm{B}$ experimentally determined. We briefly describe first the method of evaluating the age of cosmic rays using a radio active tracer and then summarize the present status in this subject.

Since the estimate of leakage life time depends upon the propagation model employed in determining the flux of radio nuclei and their decay product, we shall derive some useful relations for the flux of nuclei using slab model and steady state model. Let radio nuclei of the type $i_{m}$, where the subscript denotes the given isotope of element $i$, decay to nuclei $1_{m}$ with a mean decay time $T$ at rest. We shall assume that the attenuation mean free path $\Lambda_{1}$ due to interaction is the same for all isotopes of the element 1 . In order to take into account the decay 
probability of the radio nuclei, one has to add another term in the general diffusion equation. In the case of slab model we can add a term $f(E, X) / s(E)$ to Equation 3.23, where $s(E)=T c_{\beta \gamma}$, and the solution can be written as

$$
\begin{aligned}
j_{1_{m}}(E, x) & =\sum_{k>1} o^{f}\left\{\left|w_{1_{m}}\left(E^{\prime}, x^{\prime}\right)\right| /\left|w_{i_{m}}(E)\right|\right\}\left\{j_{k}\left(E^{\prime \prime}, x^{\prime}\right) / \lambda_{k}\left(E^{\prime \prime}\right)\right\} \\
& P_{i_{m} k}\left(E^{\prime \prime}\right) \exp \left[-f_{x^{\prime}} \int^{x}\left\{1 / \Lambda_{1}\left(E^{\prime} z\right)+1 / s\left(E^{\prime}\right)\right\} d z\right] d x^{\prime}
\end{aligned}
$$

Here, $c \beta$ and $\gamma$ are the velocity and Lorentz factor of the particle respectively Simflarly, the flux of $1_{\mathrm{m}}$ nuclet which are produced in interstellar space, directly from fragmentation of heavier elements and also through the decay of $i_{m}$, can be written as

$$
\begin{aligned}
j_{\ell}(E, x) & =\int_{0}^{x}\left\{\left|w_{\ell}\left(E^{\prime}, x^{\prime}\right)\right| /\left|w_{\ell}(E)\right|\right\} \sum_{k>\ell}\left\{j_{k}\left(E^{\prime \prime}, x^{\prime}\right) P_{\ell_{m} k}\left(E^{\prime \prime}\right) / \lambda_{k}\left(E^{\prime \prime}\right)\right. \\
& \left.+j_{\ell}\left(E^{\prime \prime}, x^{\prime}\right) / s\left(E^{\prime \prime}\right)\right\} \exp \left\{-\int_{x}^{x^{\prime}} d z / \Lambda_{\ell}\left(E^{\prime}, z\right)\right\} d x^{\prime}
\end{aligned}
$$

The first term in the above equation is same as the secondary production term in Equation 3.24 and $j_{i_{m}}\left(E^{\prime \prime}, x^{\prime}\right)$ in the second term is defined by Equation 4.26. n the case of steady state model, the flux of nuclei $j_{i_{m}}$ (E) can be obtained from Equation 3.26 by introducing an additional attenuation due to decay. Thus

$$
\begin{aligned}
j_{i_{m}}(E)= & \left.\frac{\rho \beta c}{4 \pi} \int_{0}^{\infty} w_{i_{m}}\left(E^{\prime}, x\right)|/| w_{i_{m}}(E) \mid\right\} Q_{i_{m}}\left(E^{\prime}\right) \exp \left\{-f_{0}^{x}\right. \\
& {\left.\left[1 / \Lambda_{i}\left(E^{\prime}, y\right)+1 / x+1 / s\left(E^{\prime} !\right)\right] d y\right\} d x }
\end{aligned}
$$


Where $Q_{1_{m}}(E) d x$ is the production spectrum of the nucleus $1_{m}$ per unit gram of interstellar matter, which can be estimated from Equation 4.19. Similarly, $\rfloor_{1_{\mathrm{m}}}$ (E) can also be written as

$$
\begin{aligned}
J_{\ell}(E)= & \frac{\rho \beta c}{4 \Pi} x^{\delta^{0}}\left\{\left|w_{\ell}\left(E^{\prime}, x\right)\right| /\left|w_{\ell}(E)\right|\right\} Q^{\prime} \ell_{m}\left(E^{\prime}\right) \exp \left\{-f_{0} f^{x}\right. \\
& {\left.\left[1 / \Lambda_{\ell}\left(E^{\prime}, y\right)+1 / x\right] d y\right\} d x }
\end{aligned}
$$

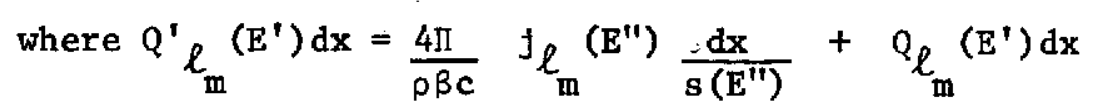

Here, $E^{\prime \prime}$ is the energy of the particle when it gives rise to a particle $\ell_{m}$ of energy $E^{\prime}$ and $Q_{\ell_{\mathrm{m}}}\left(E^{\prime}\right)$ is the production spectrum of $\ell_{\mathrm{m}}$ nucleus directly through interactions of cosmic ray nuclei. At relativistic energies when $\Lambda_{1} \dot{z} \Lambda_{\ell}$ Equation 4.29 simplifies to

$$
j_{\ell}(E)=\frac{\rho \beta c}{4 I} x \Lambda_{\ell}\left\{Q_{\ell_{m}}+Q_{i_{m}} /\left(1 / s(E)+1 / x+1 / \Lambda_{i}\right)\right\} /\left(\Lambda_{\ell}+x\right) \quad 4.31
$$

In principle, one can make use of the above equations to calculate the ratio of $\mathrm{Be} / \mathrm{B}$ nuclei as a function of energy be replacing $s(E)$ by $T \gamma X / \tau$ for various values of $\tau$ and compare it directly with the observations. However, the present experimental uncertainties do not permit such a comparison to estimate the exact age. Hence it is a general practice to compare the experimental results to the calculated ratio by considering only two possibilities, namely, (i) complete survival of ${ }^{10} \mathrm{Be}$ which gives $\mathrm{T}<<\gamma$ and (ii) complete decay of ${ }^{10} \mathrm{Be}$ which gives $\mathrm{T} \gg \tau \gamma^{2}$

In Figure 4.22 are plotted the avallable experimental data on $\mathrm{Be} / \mathrm{B}$ as a function of mean energy (Casse et al., 1971; Brown et a1., 1973a; 


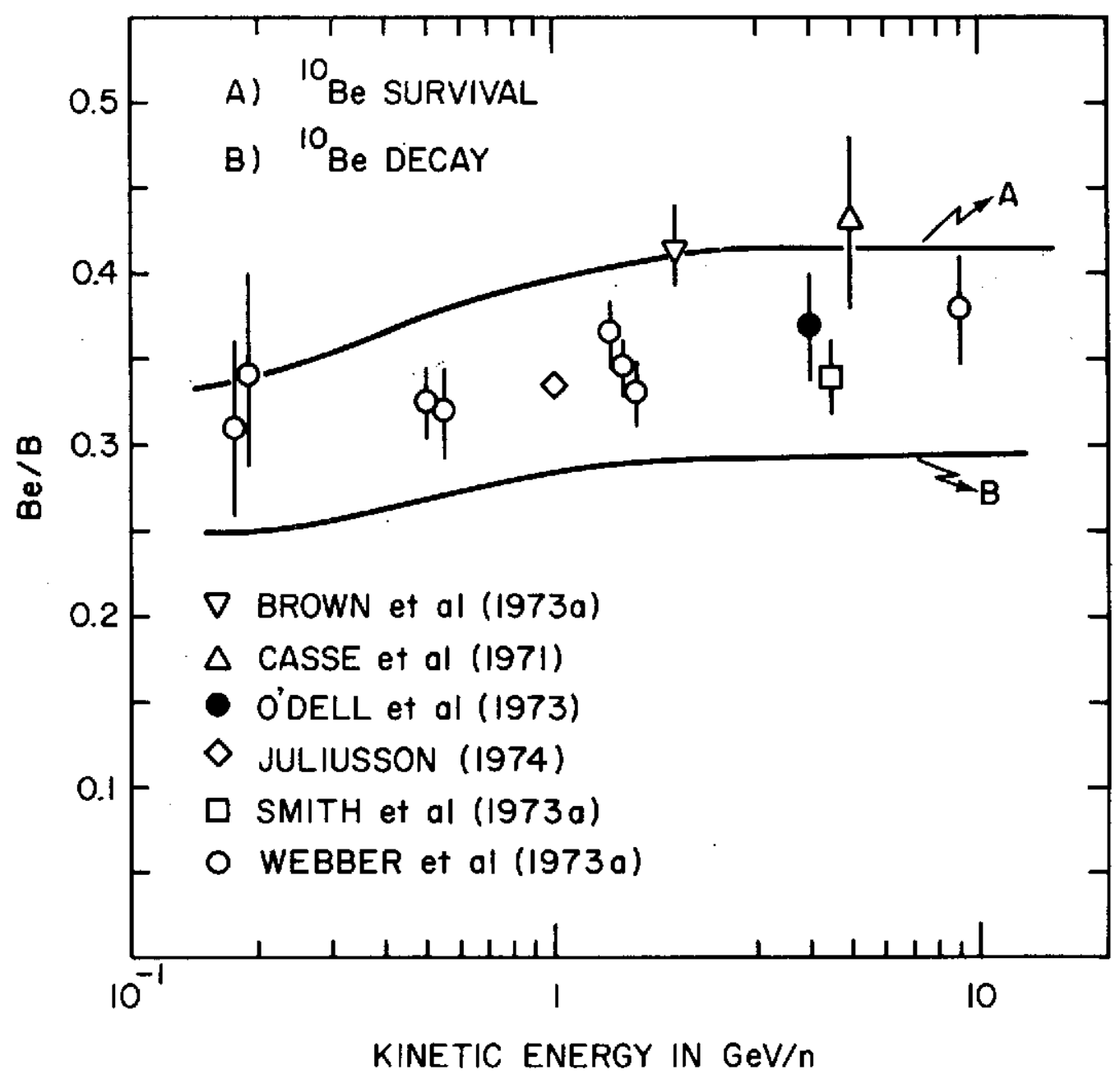

Fig. 4.22: The observed ratios of $\mathrm{Be} / \mathrm{B}$ at the top of the atmosphere are plotted as a function of kinetfc energy per nucleon; for comparison are shown Curves A and $B$, the expected ratios for complete survival and decay respectively of $10^{\circ} \mathrm{Be}$ using steady state mode1. 
o'De11 et al., 1973; Smith et al., 1973a; Webber et a1., 1973a; Juliusson, 1974). Also shown in the figure are two curves $A$ and $B$ representing the calculated values of the ratio for complete survival and decay respectively

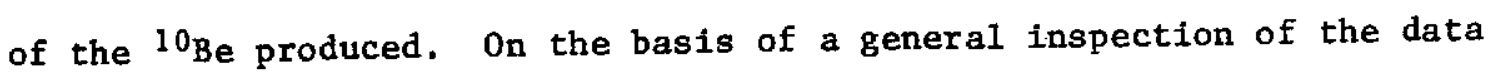
points and the associated errors in Figure 4.22, one is inclined to infer that no meaningful conclusion can be made about the life time of the radiation. Nevertheless, O'Dell et al $(1971,1973)$ have averaged the individual values obtained by some workers and claimed that there is substantive evidence to suggest a life time less than a few million years. But Raisbeck and Yiou (1973) have made a careful assessment of the various statistical and systematic errors associated with the experimental and estimated cross-sections used in these calculations, and have arrived at the conclusion that with the existing errors, no meaningful inference on the : fe time of cosmfc rays can be made from the study of the elemental bundance of $\mathrm{Be}$ and $\mathrm{B}$.

other recent experimental data which lead to small values for $\tau$. are: (a) Price et a1 (1971) from a study of the abundance ratio of nuclei with $\mathrm{Z}>85$ to those with $70 \leq \mathrm{Z} \leq 83$ conclude that the life time of cosmic rays is $<10^{7} \mathrm{yrs}$ and possibly as $10 \mathrm{w}$ as $10^{6} \mathrm{yrs}$; (b) since the nucleus ${ }^{53} \mathrm{Mn}$ decays only by K-capture with a mean life on two million years, Reames (1971) has concluded from an analysis of the observed ratio of $\mathrm{Cr} / \mathrm{Mn}$ as a function of energy, that the cosmic ray age is consistent with the confinement of cosmic rays in the Disk rather than in the Halo.

While all attempts in the past to use ${ }^{10} \mathrm{Be}$ as a clock to measure the 1 ife time of cosmic rays have been confined to the study of the ratio $\mathrm{Be} / \mathrm{B}$, the ultimate aim will be to study the relative intensitives of the isotopes, 
where the effect of the ${ }^{10}$ Be decay will be maximal and will not be diluted as in the case of the ratio of Be to $B$ nuclei. With the growing capability and sophistication of detector instrumentation, such intrinsic resolution needed for the efficient separation of individual isotopes of $\mathrm{Be}$ and $\mathrm{B}$ nuclei has just become a reality (Garcla-Munoz et al, 1973; Webber et al, 1973a). These first measurements have not yet been able to lead to definitive results but have clearly demonstrated that in the near future we will have a reliable estimate of the cosmic ray life time.

\subsection{Anti-Nuclei in Cosmic Rays}

It is well known fact that detection of antiparticles in cosmic radiation is perhaps the only direct proof to the existence of antimatter outside the solar system. After the early measurements of the east-west effect (Johnson, 1938), which showed that cosmic rays composed of positively charged particles, the major attempts to look for anti-nuclei during late fifties gave an upper limit of $0.1 \%$ at energies less than a few hundred MeV/n (Aizu et al, 1961; Grigorov et al, 1961). Nonetheless, annihflation of antimatter with matter has often been suggested as an energy source in those astrophysical objects for which nucleosynthesis or gravitation may be inadequate. Such a process to occur on a large scale in the baryon-symetric Universe has been invoked by stecker et al. (1971) to explain the diffuse component of the background gamma ray spectrum. Further, Alfven (1965) proposed a model allowing the co-existence of matter and anti-matter within our own galaxy. As a result of all these, systematic search for anti-nuclei has been undertaken over a wide range of energies using magnetic spectrometers. In this section we brlefly summarize the available experimental results and the theoretical estimates of the antiproton flux of secondary origin in the Galaxy. 


\subsubsection{Ant1-Nuclei of Secondary Origin}

When high energy cosmic ray nuclei interact with interstellar gas, baryon pairs are also created along with other hadrons. Hence, even in the absence of anti-matter in the Universe, one would expect to detect anti-protons $(\overline{\mathrm{p}})$ in cosmic rays; indeed the presence of positrons in cosmic radiation is known well for many years. Many theoretical estimates of the fraction of $\bar{p}$ in cosmic radiation have been made in the past, of which the early ones were only speculative in nature (Fradkin, 1956; Burbridge and Hoyle, 1957; Hayakawa et al. 1958). With the availability of the experimental cross-sections for the production of $\bar{p}$ over a wide range of energies, more detailed calculations have been attempted to determine the equilibrium ratio of $\bar{p} / p$ as a function of energy using various propagation models for cosmic rays (Rosen, 1967; Shen and Berkey, 1968; Wayland and Bowen, 1968; Suh, 1971; Chen, 1972; Gaisser and Maurer, 1973; Badhwar et al, 1973). The production spectrum $\frac{Q_{p}}{p}(E) d E$ per $\left(\mathrm{cm}^{3} \mathrm{sec}\right)$ can be written from Equation 4.19 as

$$
\left.Q_{\bar{p}}(E) d E=4 \pi n f_{E,}^{\infty} d E^{\prime}\right\}\left(E^{\prime}\right) \sigma_{\bar{p}}\left(E^{\prime}\right) \psi_{\bar{p}}\left(E^{\prime}, E\right) d E
$$

where $\mathrm{E}$, is the threshold for the production of a baryon pair, which is about $6 \mathrm{GeV}$ in $\mathrm{p}-\mathrm{p}$ interactions and $\mathrm{n}$ is the number of interstellar gas

atoms $\mathrm{cm}^{-3}$. From the avallable accelerator data, the cross-section $\sigma\left(E^{\prime}\right)$ for the production of $\bar{p}$, ineluding the enltipliclty, can be approximated (Badhwar et a1, 1973) as $\sigma_{\bar{p}}(\mathrm{E})$ : $9.26 \times 10^{-3}(\mathrm{E}-\mathrm{C})$, where $\mathrm{C}=10.8 \mathrm{GeV}$ is the effective threshold energy; this relation seems to be valid up to about $500 \mathrm{GeV}$. The energy distribution function $\psi$ is given by Shen and Berkey 
(1968) as

$$
\psi_{\vec{p}}\left(E^{\prime}, E\right) d E=\Pi E \exp \left(-\Pi E^{2} / 4<E>2\right) / 2<E>2
$$

where $\langle E\rangle$ is the mean energy of $\bar{p}$, which is $\alpha E^{\prime}$ on the basis of scaling hypothesis and is $\alpha E^{13 / 4}$ according to statistical model. Knowing the production spectrum of $\overline{\mathrm{p}}$. one can then calculate the equilibrium spectrum using any propagation model. This spectrum then can be compared with the equilibrium proton spectrum to obtain the ratio $\bar{p} / \mathrm{p}$ as a function of energy.

In Figure 4.23, we show the calculated equilibrium ratio $\bar{p} / \mathrm{p}$ as a function of energy (Gaisser and Maurer, 1973; Badhwar et a1, 1973) for a mean amount of $5 \mathrm{~g}$. $\mathrm{cm}^{2}$ of matter traversed by cosmic rays in interstellar space. Since the effective threshold energy for production is about $10 \mathrm{GeV} / \mathrm{n}$, the flux of antiprotons of energy less than <E> decreased while proton flux continues to increase, resulting in a rapid decrease of the ratio below about $2 \mathrm{GeV}$. Further, the uncertainty in this energy region is rather large and depends upon the exactness of the Equation 4.33 for values of $\mathrm{E}$ much smaller than $\langle\mathrm{E}\rangle$, the propagation model and the uncertainty in the equilibrium intensity of protons in interstellar space; however, the experimental upper 1imit of $3 \times 10^{-4}$ at energies <200 MeV (Apparao, 1967) is about a few order of magnitude larger than the calculated value. At higher energles, the calculation of Gaisser and Maurer shows that the ratio approaches an asymptotic value of about $4.6 \times 10^{-4}$. It has been pointed out by Badhwar et al that $\overline{\mathrm{p}} / \mathrm{p}$ ratio is dependent on whether the matter traversed by cosmic rays is during or after the acceleration of particles to cosmic ray energies. We also show in this figure the only one available upper limit above a GeV (Bogomolov et a1, 1971) and one notes that this 1imit 


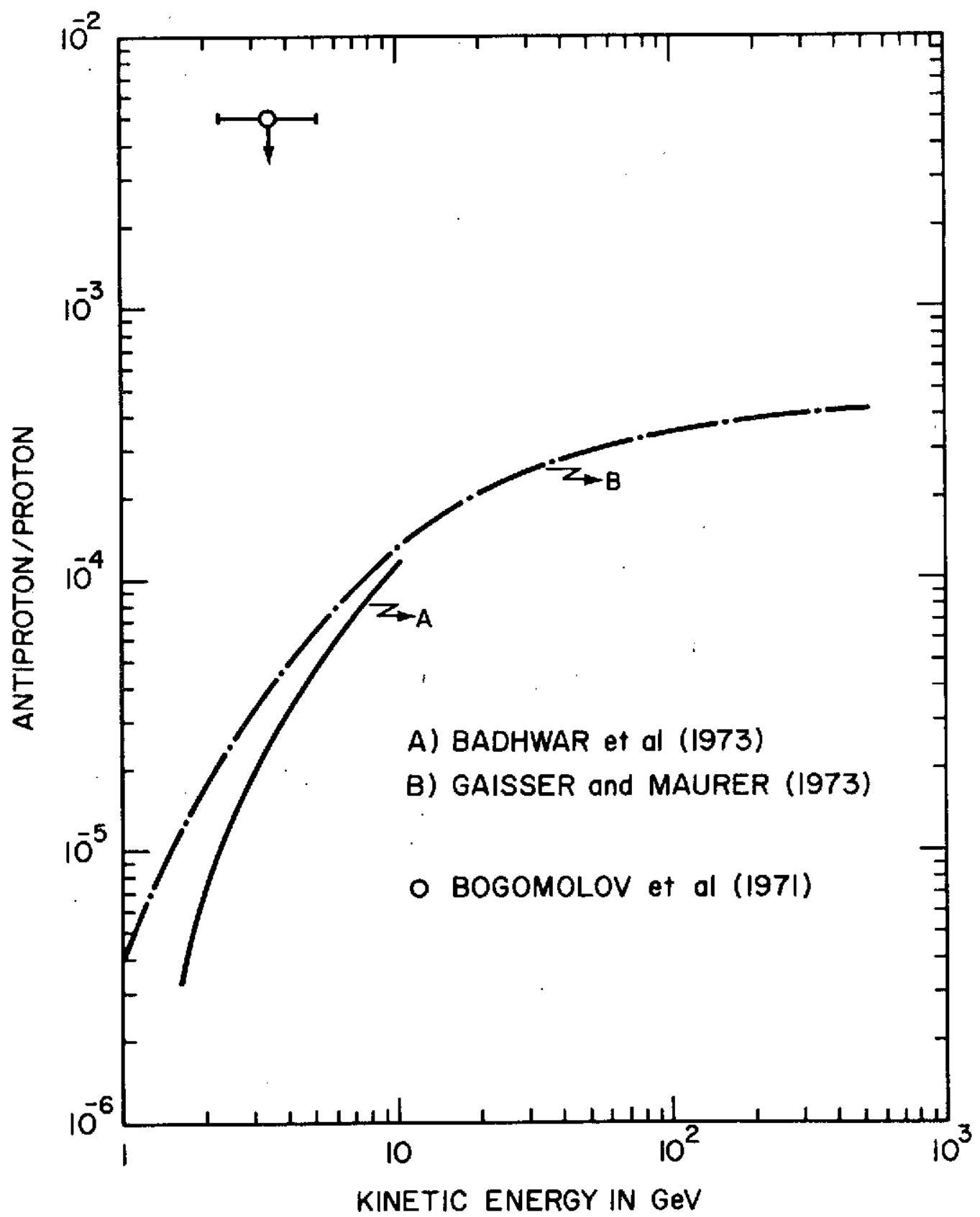

Fig. 4.23: The equilibrium ratio of $\bar{p} / p$ in a steady state model with $\mathrm{X}=5 \mathrm{~g} \mathrm{\textrm {cm } ^ { - 2 }}$ is shown as a function of kinetic energy. 
needs to be improved by at least two orders of magnitude to make meaningful comparison with the theory.

\subsubsection{Anti-Nuclei of Primary Origin}

As is evident from the preceding section, the detection of antiprotons in cosmic radiation cannot unambiguously prove the existence of antimatter in cosmic space before one could separate those resulting from high energy interaction. Hence, most of the experiments carried out so far are aimed at detecting antinuclei of $\mathrm{Z} \geq 2$. In Table 4.5 , we show the available results from such studies as summarized by Garcia-Munoz (1973). One may conclude from this that the fraction of antimatter, if it exists in the Galaxy, is less than $2 \times 10^{-4}$ of the normal matter. Though it is interesting to set a more stringent upper limit to this ratio by a few orders of magnitude, the present result itself might perhaps indicate that in the baryon-symmetric universe, matter and antimatter might have separated from each other on an extragalactic scale. Would that then tempt us to look for anti-matter at energies $>10^{16} \mathrm{GeV}$, where one would expect antimatter to reach us from distant galaxies in the Universe? 
Table 4.5

Upper Limits to the Ratio of Ant1-Nuclei to Nuclei In : Cosmic Radiation

\begin{tabular}{|c|c|c|c|c|}
\hline Nuclei & RIgidity/e & nergy range & Upper Limits & Reference \\
\hline$Z=2$ & $0.2-4.3$ & $\mathrm{GeV} / \mathrm{n}$ & $1.4 \times 10^{-3}$ & Eavans on (1972) \\
\hline$z^{22}$ & $5-33$ & GV & $2 \times 10^{-4}$ & Smoot et a1 (1973) \\
\hline$z^{\geq} 2$ & $33-100$ & $"$ & $1.5 \times 10^{-2}$ & Smoot et al (1973) \\
\hline$z \geq 3$ & $4-125$ & $"$ & $3 \times 10^{-3}$ & Golden et al (1973b) \\
\hline$z \geq 3$ & $4-10$ & $"$ & $8 \times 10^{-3}$ & Golden et al (1973b) \\
\hline$Z \geq 3$ & $10-50$ & $"$ & $5 \times 10^{-3}$ & Golden et al (1973b) \\
\hline$z \geq 3$ & $60-125$ & $"$ & $6 \times 10^{-2}$ & Golden et al (1973b) \\
\hline$z \geq 2$ & $15-30$ & $"$ & $9 \times 10^{-3}$ & Verma et al (1972) \\
\hline $\mathrm{z} \geq 2$ & $30-50$ & $"$ & $2.6 \times 10^{-2}$ & Verma et a1 (1972) \\
\hline $\mathrm{z}^{2} 2$ & $50-100$ & $"$ & $7 \times 10^{-2}$ & Verma et al (1972) \\
\hline $\mathrm{Z} \geq 6$ & $<200$ & $\mathrm{MeV} / \mathrm{n}$ & $6 \times 10^{-3}$ & Ivanova et al (1968) \\
\hline $\mathrm{z} \geq 6$ & $5-9$ & $\mathrm{GeV} / \mathrm{n}$ & $7.5 \times 10^{-2}$ & Greenh 111 et al (1971) \\
\hline
\end{tabular}




\section{Cosmogenic Electromagnetic Radiations in the Galaxy}

\subsection{Introduction}

The motion of cosmic rays within the confines of the Galaxy would necessarily imply their interaction with matter, magnetic fields and radiations existing in interstellar space. Some of these interactions lead to the emission of electromagnetic radiations which may fall anywhere in the spectrum ranging from the radio regime to the gamma ray regime. Though the rate at which cosmic ray particles lose energy in this manner Is exceedingly small, their integrated effect becomes of considerable significance under cosmic conditions of space and time. Furthermore, a distinguishing feature of these emitted electromagnetic radiations is that they originate from all regions of galactic space permeated by cosmic rays and hence lead to a diffuse glowing or sky background in the relevant frequency region. It is also evident, that as viewed from the solar neighborhood, the intensity of these galactic background radiations as a function of celestial directions will exhibit a close correlation with galactic coordinates. One may also anticipate the possible existence of a background radiation of extragalactic origin. However, in contrast to the former, the characteristic feature of the extragalactic radiation will be its isotropic nature. Observationally, the two components will be superimposed on one another and it will be necessary to resolve the two contributions before they can be interpreted.

Cosmic ray interactions in galactic space leading to background electromagnetic radiations are of three kinds: they are interactions with Interstellar matter, radiation fields and magnetic fields. The 
intensity of such a radiation received by a terrestrial observer from any direction In the Galaxy, defined by the galactic longitude 1 and latitude $b$, can be written as

$$
I_{\gamma}(E) d E=\frac{d E}{4 \pi} \int_{0}^{L(1, b)} e^{-f(E, s)} \psi_{\gamma}(E, s) d s \text { photons } \mathrm{cm}^{-2} \operatorname{sr}^{-1} \sec ^{-1} 5.1
$$

where $\psi_{\gamma}(E, s) d E$ is the emissivity at a distance $s$ through any of the processes mentioned above, $f(E, s)$ is the optical depth and $L(1, b)$ is the total distance along the line of sight in the Galaxy. In the case of the galactic Disk, Equation 5.1 is valid for telescopes having a resolution $\theta_{\mathrm{m}}$ such that $\tan \theta_{m} \leq z_{o} / L(1, b)$, where $z_{0}$ is the thickness of the Disk. For larger values of $\theta_{\mathrm{m}}$, (see F1gure 5.1 ), one can rewrite Equation 5.1 by expressing the Intensity from the Disk as

$$
\begin{gathered}
\phi(E) d E=\frac{d E}{4 \pi} \int_{-\theta}^{\theta}{ }_{m}^{/ 2} \cos b d b \int_{s=0}^{L(1, b)} e^{-f(E, s)} \psi_{\gamma}(E, s) d s \text { photons. } \\
\mathrm{cm}^{-2} \mathrm{rad}^{-1} \mathrm{sec}^{-1}
\end{gathered}
$$

The value of $L(l, b)$ can be obtained from F1gure 5.1 as

$$
\begin{aligned}
\mathrm{L}(1, \mathrm{~b}) & =\mathrm{L}(1) / \cos \mathrm{b} \text { for } \mathrm{L}(1) \tan \mathrm{b}<\mathrm{z}_{\mathrm{o}} / 2 \\
& =\frac{1}{2} \mathrm{z}_{0} / \sin \mathrm{b} \text { for } \mathrm{L}(1) \tan \mathrm{b}>\mathrm{z}_{\mathrm{o}} / 2
\end{aligned}
$$

and $L(1)=\left\{R^{2}+R^{2}-2 R o\left[R o \operatorname{Sin}^{2} 1-\operatorname{Cos} 1\left(R^{2}-R^{2} \operatorname{Sin}^{2} 1\right)^{\frac{1}{2}}\right] \quad 5.4\right.$ Using this general formulation, we shall estimate the intensity of various galactic background radiations arising through the respective physical pro-. cesses. We will then compare them with observations and therefrom deduce information on the astrophysical conditions in the Galaxy. 


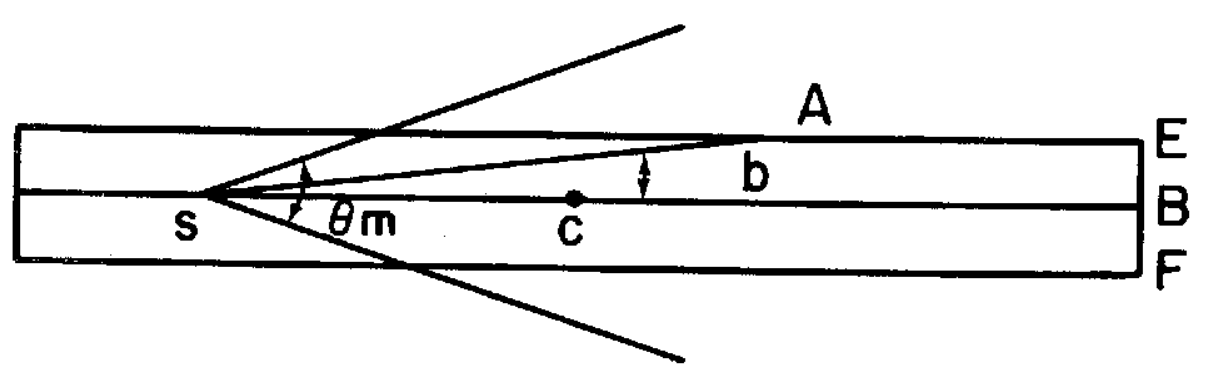

$$
S C=R_{0}, C B=R, E F=z_{0}, S A=L(l, b)
$$

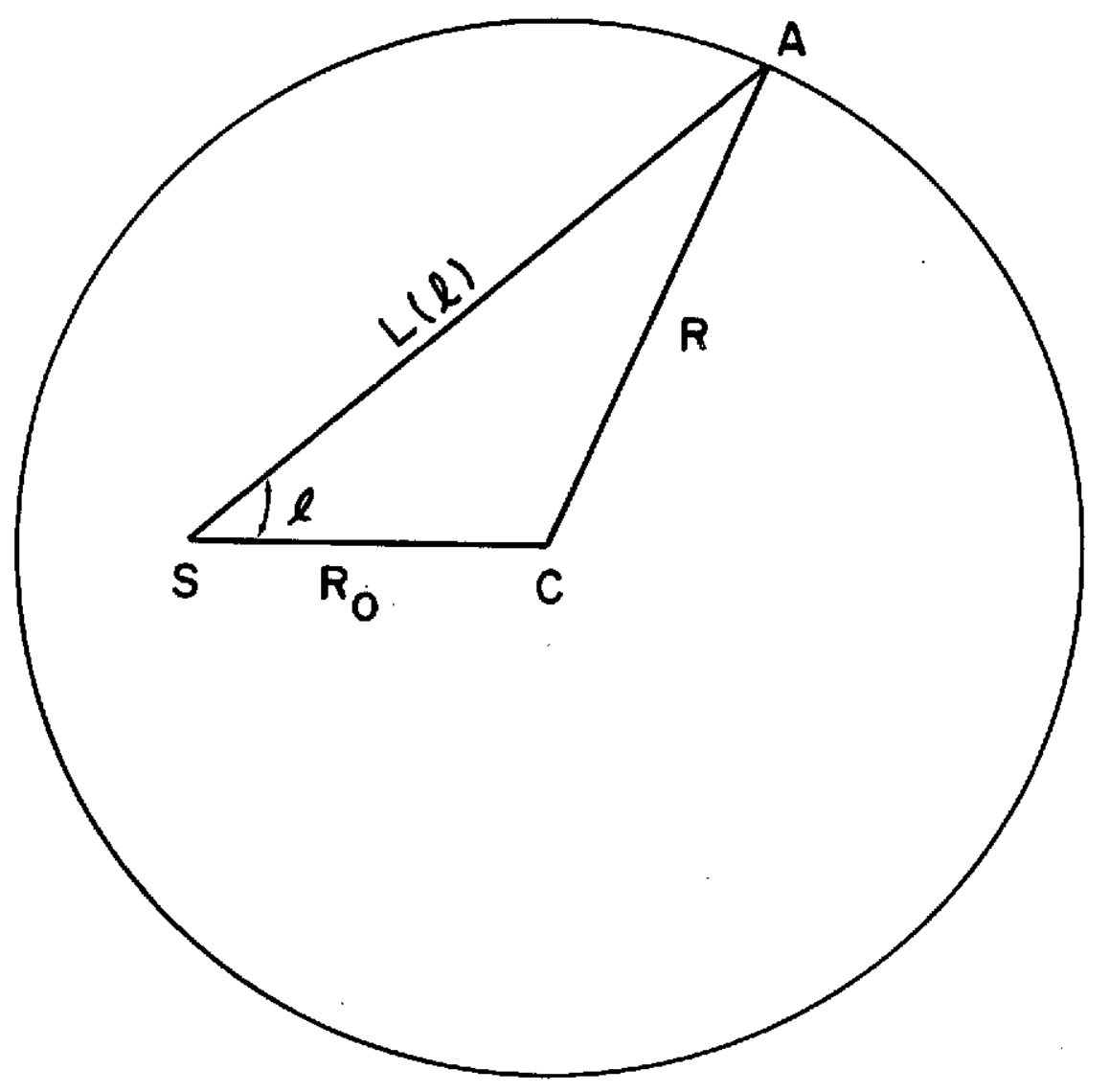

$$
\text { S-SUN, C-CENTRE }
$$

Fig. 5.1: Schematic drawing of the Galaxy and the detector opening angle to derive the geometrical parameters in Equations 5.3 and 5.4 . 


\subsection{Cosmic Ray Interactions with Interste1lar Gas}

In principle there are many physical processes involving cosmic ray Interactions with intersteliar gas resulting in the emission of electromagnetic radiations. To name a few: (1) Greenberg (1969) has discussed the radio emission lines produced by electron cascades following recombination to highly excited states; (11) the characteristic x-ray lines from transitions following inner shell ionization of ambient nuclei by cosmic rays have also been studied (Gould and Burbidge, 1963; Hayakawa and Matsuoka, 1964; Lampton et al., 1971; Verma, 1971);(i1i) S11k and Steigman (1969) have estimated the doppler-broadened Lyman $\alpha$-like radiations emitted by low energy cosmic ray nuclei while capturing electrons into excited states followed by the cascading to the ground state; and (iv) nuclear interactions can result in the excitation of the incident or target nucleus which while reverting to its ground state emits gamma rays. In all these processes, except perhaps (iv), the contributions originate from interactions of very low energy cosmic rays, about which we know very little at present.

of the two processes which make significant and calculable contributions of gamma rays, the first relates to the decay of neutral pions created in nuclear interactions of cosmic rays of energy above a few hundred $\mathrm{MeV} / \mathrm{n}$ with interstellar matter. The second arlses through the bremsstrahlung of cosmic ray electrons in electromagnetic interactions with nuclei of interstellar atoms. These two will now be discussed in some detail. 


\subsubsection{Plonic gamma rays}

When cosmic ray nuclet, having energies in excess of the threshold for pion production, interact with interstellar matter, gamma rays result from the decay of neutral pions created in such collisions; a small part also arises from the decay of $\Sigma$ hyperons and no mesons. From a knowledge of the pion production spectrum, the interstellar cosmic ray spectrum and the matter density at $s$, one can write the emissivity function $\psi(E, s) d E$ defined in Equation 5.1 associated with this process as

$$
\psi_{Y}(E, s) d E=8 \pi n_{H}(s) d E \int_{\varepsilon}^{\infty} \frac{d E}{\sqrt{E_{\pi}^{2}-m_{\pi}^{2}}} \int_{E 1}^{\infty} j_{p}\left(E_{p}, s\right) \sigma_{\pi}\left(E_{\pi}, E_{p}\right) d E p .5 .5
$$

Here $\varepsilon=E+m_{\pi}^{2} / 4 E ; n_{H}(s)$ is the neutral hydrogen density at $s ; j_{p}\left(E_{p}, s\right)$ is the cosmic ray proton $f l u x$ at $s$; and $\sigma_{\pi}\left(E_{\pi}, E_{p}\right)$ is the cross-section for the production of $\pi^{\circ}$ mesons defined in this case as

$$
\sigma_{\pi}\left(E_{g} E_{p}\right)=\sum_{k} \sum_{i} \sigma_{\pi}\left(E_{\pi}, E_{k}\right) g\left(E_{k}, E_{p}\right) f_{k} f_{1}
$$

The summation is carried over all interactions of cosmlc ray nuclei of the type $k$ wth interstellar nuclei of the type $1 ; f_{k}$ is the fraction of cosmic ray nuclei of the type $k$ to that of protons, $f_{1}$ is the relative abundance of interstellax nuclei with respect to hydrogen and $g\left(E_{k}, E_{p}\right)$ is a function which takes care of the energy thresholds for different nucleus-nucleus collisions: It can be easily shown that the pionic gamma-ray spectrum should exhibit a maximum at an energy of about $67.5 \mathrm{MeV}$, corresponding to half the rest mass of the neutral pion. Extensive attempts made to evaluate Equation 5.5 by different authors (Stecher, 1970, 1971; Cavallo and Gould, 1971; Levy and Goldsm1th, 1972) differ by a factor $; 2$ in the integral 
intensity of gamma rays above $100 \mathrm{MeV}$, and hence there could exist a significant uncertainty in the estimated differential spectrum. In Figure 5.2 is shown the differential intensity of gamma-rays for an interstellar gas density of one hydrogen atom $\mathrm{cm}^{-3}$. In this figure, the spectrum below a $\mathrm{GeV}$ is taken from Stecker (1970), which is then extended to higher energies with a spectral index of $-2,6$. The constant spectral index above a GeV is justified by the fact that the observed spectrum of gamma rays in the atmosphere has a spectral index of -2.6 (Stephens, 1970a; Anand et al, 1973b). It might also be mentioned that the East-West asymmetry of atmospheric gamma rays over low latitudes suggests the strong influence of the isobaric decay as the source for the gamma rays (Stephens, 1970b).

In order to estimate the intensity of gamma rays towards any direction of the Galaxy, one has to substitute Equation 5.5 in Equation 5.1. Since absorption of gamna rays in interstellar space is negligible one can rewrite Equation 5.1 as

$$
I_{\gamma}(E) d E=\frac{\psi_{Y}(E) d E}{4 \pi} \int_{0}^{L(1, b)} \cdot n_{H}(s) \rho_{C \cdot R}(s) d s
$$

where $\psi_{\gamma}(E)$ is plotted in Figure $5.2 ; \rho_{C} . R(s)$ is the cosmic ray density as normalized to that observed in the neighborhood of the Earth. If one assumes a mean value for the cosmic ray density and the gas density, the above integral reduces to $\psi_{\gamma}(E) d E / 4 \pi<n_{H} \rho_{C} . R^{L}>$. In consequence if one observes a finite flux of cosmic gamma rays in the Galaxy, one can in principle estimate the quantity $\left\langle\mathrm{n}_{\mathrm{H}} \rho_{\mathrm{C} . \mathrm{R}} \mathrm{L}^{\mathrm{L}}>\right.$ along that direction. Since bremsstrahlung radiation leads to a similar deduction, we shall compare the calculated spectra with the observed data after dealing with the bremstrahlung gamma rays as well. 


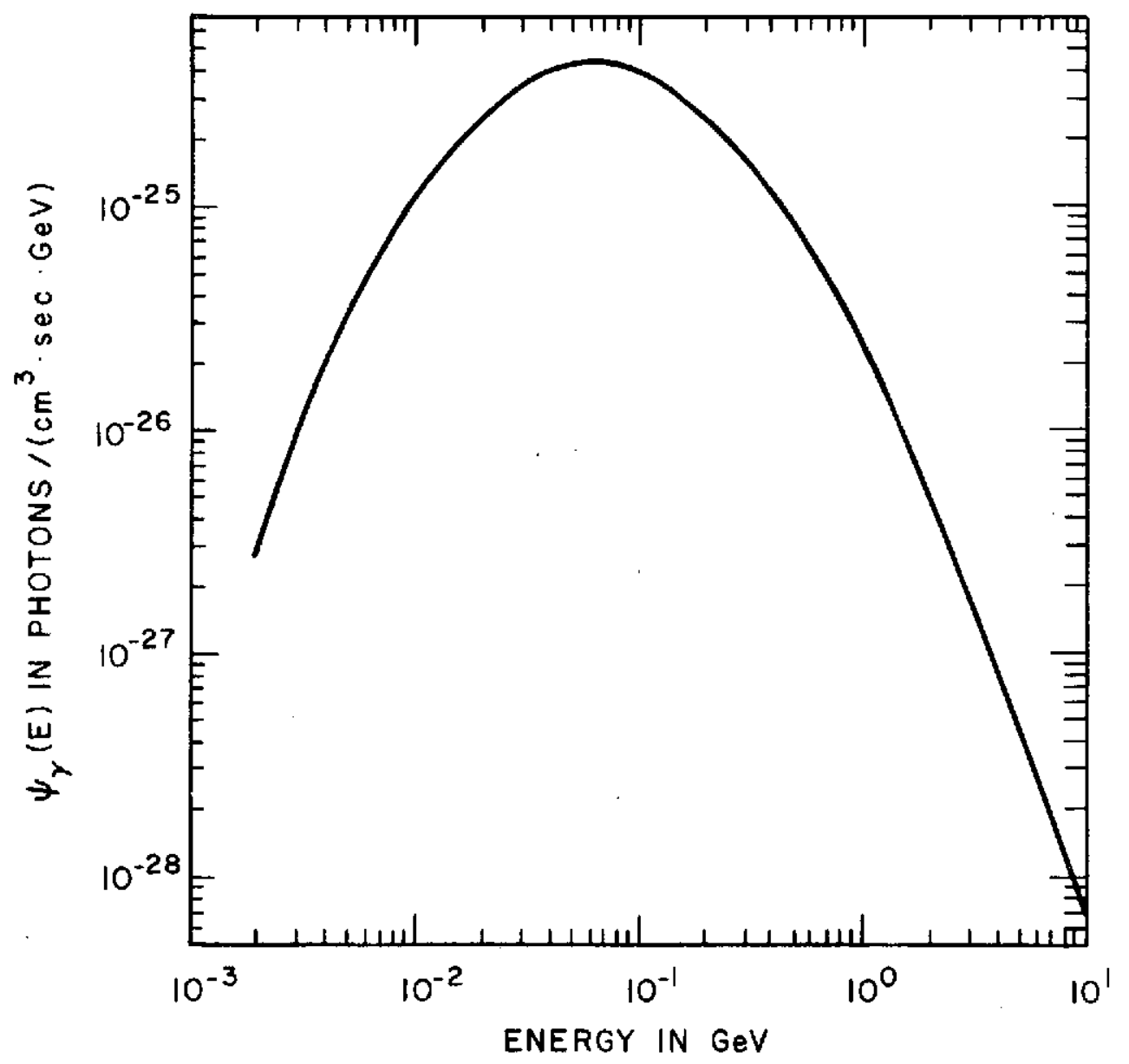

Fig. 5.2: Production spectrum of pionic gamma rays in interstellar space per hydrogen atom. 


\subsubsection{Bremsstrahlung radiation}

When cosmic ray electrons interact with interstellar gas, they give rise to bremsstrahlung radiation whose emissivity can be written as

$$
\psi_{\gamma}(E, s) d E=4 \pi n_{H}(s) d E \int_{E}^{\infty} j_{e}\left(E^{\prime}, s\right) \sigma_{\gamma}\left(E, E^{\prime}\right) d E
$$

where $j_{e}(E)$ is the equilibrium spectrum of galactic electrons as discussed In section $4.5 ; \sigma_{\gamma}\left(E, E^{\prime}\right)$ is the differential cross-section for the production of gamma rays of energy $E$ from an electron of energy $E^{\prime}$ summed up over all nuclel of the amblent gas. At energies greater than a few GeV, where the interstellar electron spectrum is a simple power law, the emissivity can be written as (Stephens, 1969)

$$
\psi_{Y}(E, s)=2.8 \times 10^{-27} \rho_{C .} R_{H}(s) E^{-2.62} \text { photons } \mathrm{cm}^{-3} \mathrm{sec}^{-1} \mathrm{GeV}^{-1}
$$

Comparing this with Figure 5.2, one finds that this contribution is an order of magnitude smaller than the pionic gamma rays. The bremsstrahlung spectrum below a GeV flattens since the equilibrium spectrum of cosmic ray electrons also flattens.

In Figure 5.3 are shown the integral spectra of gamma rays resulting from the interaction of cosmic rays with interstellar matter in the direction of the galactic centre (Anand and Stephens, 1972), for a typical detector with $\theta_{\mathrm{m}}=30^{\circ}$. The pionic (curve A) and bremsstrahlung (curve B) gamma ray intensities have been calculated by assuming ${ }^{\rho_{C}} \mathrm{C} . \mathrm{R}=1, \mathrm{n}_{\mathrm{H}}=1$, and an effective thickness of $z_{0}=360$ pe for the gas disk, which corresponds to a thickness of 280 pc as defined by the half density points (Section 2.3); Curve $C$ is the sum of Curves $A$ and $B$. It is seen that the bremsstrahlung radiation contributes a small fraction of the total radiation above 100 


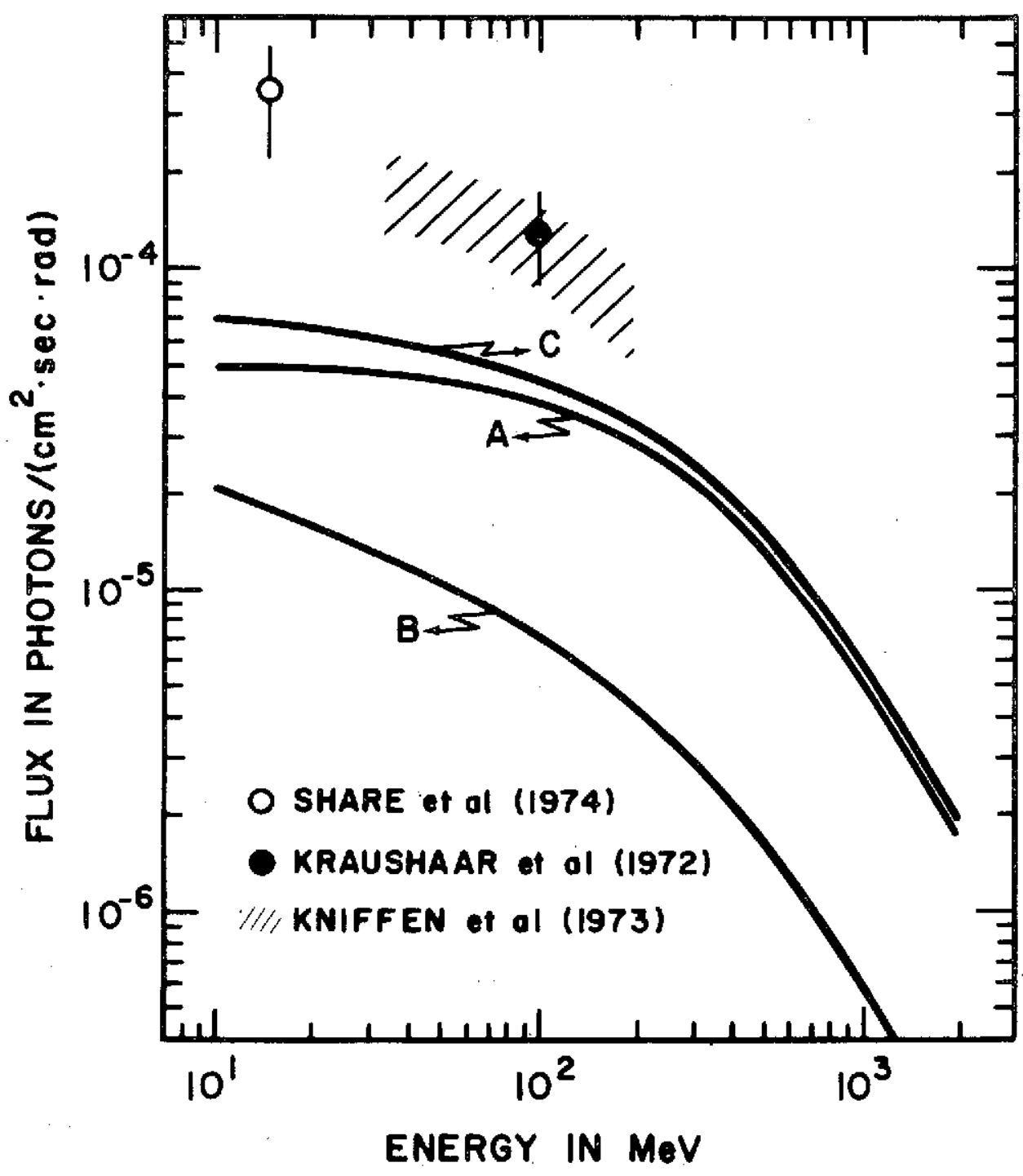

F1g. 5.3: The observed integral intensities of gamma rays towards the galactic center are compared with the calculated spectra for a detector with $\theta_{\mathrm{m}}=30^{\circ}$; Curves $A$ and $B$ respectively are the spectra of pionic and bremsstrahlung gamma rays, and Curve $C$ is the sum of Curves $A$ and $B$. 
MeV but becomes significant at lower energies. It has been shown by Maraschi et al (1968) that the estimated bremsstrahlung radiation below $1 \mathrm{MeV}$ is very much smaller than the observed radiation, even with extreme assumptions regarding the spectral shape of cosmic ray electrons at the corresponding energies. We have also shown in this figure, the recently observed flux values towards the galactic centre (Kraushaar et al,1972; Kniffen et al, 1973; Share et a1, 1974), confirming the original findings of a line emission from the galactic plane by Clark et al, (1968); there also exist some upper limits in this energy region which are in agreement with the above results (Dahlbacka et al, 1973; Bennett et al, 1972) except that of Frye et al, (1971). Though one may have to correct for the different values of $\theta_{m}$ used in these experiments in order to compare with theoretical estimates (except for the detector of Kraushaar et al, for which $\theta_{\mathrm{m}}=30^{\circ}$ ), one can infer from this figure that the observed value is a factor of about two higher than the estimated one. It has been further shown by Anand and Stephens (1972) that the central region of the Galaxy does not contribute much to this total radiation. If the observed intensity of gamma rays has to be accounted for by interaction of cosmic rays with ambient matter, then it is necessary that the value $\int_{0}^{L} n_{H}(s) \rho_{C . R}(s) d s / L \sim 2 n_{H}(0) \rho_{C} . R$. direction of the galactic centre.

Many suggestions have been put forward to understand this enhancement of the observed flux of gamma rays towards the galactic centre on the basis of cosmic ray interactions with the ambient gas (Stecker, 1969; Anand and Stephens, 1971; Ginzburg and Khazan, 1972; Strong et al, 1973; Black and Fazio, 1973, Stecker et al, 1974; B1gnami and Fichtel, 1974). In effect all these suggestions indicate that there could be an increase of cosmic 
ray density with or without a corresponding increase of matter density towards the inner region of the Galaxy because the observed flux value in the direction of the anticentre (Kraushaar et a1, 1972) is in good agreement with the estimated value in 'uat direction.

\subsection{Cosmic Ray Interactions with Radiation Fields}

When cosmic ray electrons undergo inverse-Compton scattering with ambient photons in the Galaxy, they impart part of their energy to the soft photons thereby giving rise to galactic background radiation in the $X$ - and $\gamma$-ray regions. The emissivity function in this case can be written in the form

$$
\psi_{\gamma}(E, s) d E=4 \pi d E \int_{E_{1}}^{E_{2}} n(\varepsilon, s) d \varepsilon \int_{E_{\min }}^{\infty} \sigma\left(E, E^{\prime}, \varepsilon\right) j\left(E^{\prime}, s\right) d E^{\prime}
$$

where $n(\varepsilon, s) d \varepsilon$ is the number of photons per unit volume of energy $\varepsilon$ at $s$; $f\left(E^{\prime}, s\right)$ is the flux of cosmic ray electrons; and $\sigma\left(E, E^{\prime}, \varepsilon\right)$ is the crosssection for the production of a photon of energy $E$ by an electron of energy $E^{\prime}$ scattering an ambient photon of energy $E$ and is, according to Ginzburg and Syrovatskil (1964),

$$
\sigma\left(E, E^{\prime}, \varepsilon\right) d E=\frac{4 r e^{2}}{4} \frac{E d E}{\varepsilon^{2} \gamma}\left[2-\frac{E}{\gamma^{2} \varepsilon}+4 \ln \left(\frac{E^{\prime}}{4 \gamma \varepsilon}\right)+\frac{8 \varepsilon \gamma^{2}}{E^{\prime}}\right]
$$

Here $\mathrm{r}_{\mathrm{e}}, \gamma$ and $\mathrm{m}$ are the classical radius, mass and Lorentz factor of the electron, respectively. The limits $E_{1}$ and $E_{2}$ in Equation 5.10 are defined by the energy band in which the ambient photon radiation exists and $E_{\text {min }}=$ $\mathrm{mc}^{2}(\mathrm{E} / 4 \varepsilon)^{\frac{1}{2}}$.

Estimates of the inverse Compton spectrum from the Galaxy has been made by many authors in the past (eg. Felten and Morrison, 1966; Cowsik and Pal, 1969; Shen, 1969; O'Connell and Verma, 1969; Ipavich and Lenchek, 1970; Maraschi and Treves, 1970). In Figure 5.4, the integral spectra of the inverse Compton photons in the direction of the Centre, assuming $n(\varepsilon)$ 


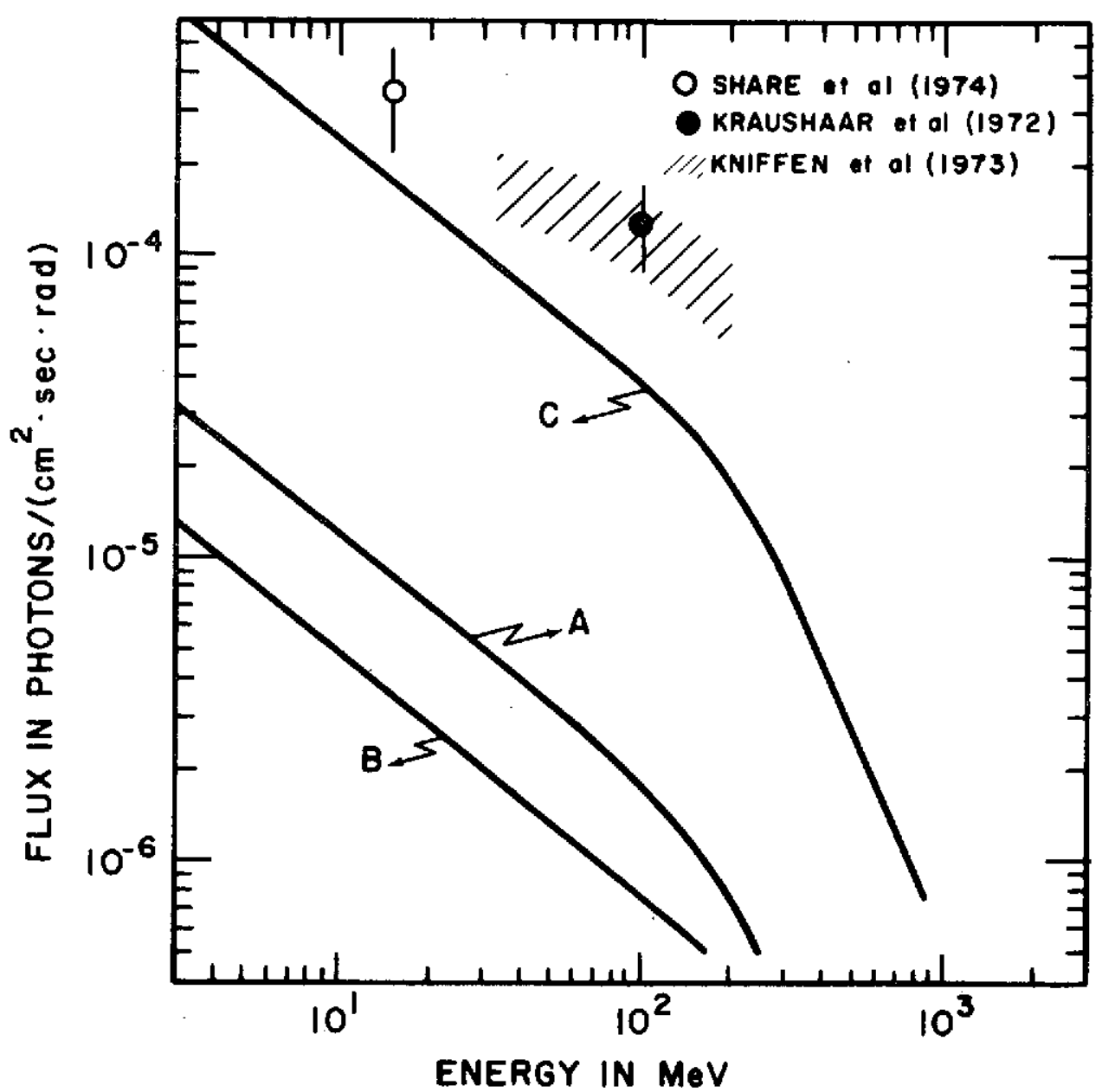

Fig. 5.4: The calculated integral spectra of gamma rays through inverse Compton scattering of black body, starlight and submillimeter photons are shown by Curves A, B and C respectively, and compared with the observations. 
and $j\left(E^{\prime}\right)$ are independent of $s$, and $\rho_{C . R}=1$, for a typical detector with $\theta_{m}=30^{\circ}$ are given (Anand and Stephens, 1972); also shown here are the observed flux values summarlzed in Flgure 5.3. Curves $A, B$ and $C$ of Figure 5.4 are the inverse Compton spectra from the Untversal Black Body photons at $2.7^{\circ} \mathrm{K}$, the star light photons and the submillimeter radiations respectively; the energy densities for these ambient photons are taken to be $0.25 \mathrm{eV}, 0.5 \mathrm{eV}$ and $6 \mathrm{eV}$ respectively. Here one has to note that the possible existence of the submillimeter radiation first reported by Shivanandan et al, (1968) is not confirmed by the recent experiments (Williamson et a1, 1973); furthermore, its existence is shown to be inconsistent with the gamma ray observations (Anand and Stephens, 1972; Cowsik, 1972). Thus, if one ignores Curve C in Figure 5.4, it is quite evident that the contribution of inverse-Compton process from the D1sk, in general, is very much smaller than that due to cosmic ray interaction with matter (Section 5.2). It may be noted that in the above calculation, it is assumed that $n(\varepsilon)$ is independent of $s$, which may not be true for the optical photons in the Galaxy. Recently, Sullivan and Cowsik (1973) showed that if the optical luminosity is proportional to the mass distribution in the Galaxy, one could account for the enhanced flux of gamma rays towards the Centre. On the other hand, it is found that if one makes use of the observed infra-red radiation from the central region of the Galaxy, the Inverse Compton process contributes only a small fraction of the observed flux (Anand and Stephens, 1972).

\subsection{Synchrotron Radiation}

Cosmic ray electrons in the Galaxy emit synchrotron radiation as they spiral along the weak interstellar magnetic field lines. This synchrotron emission is recognized to be the source of the galactic nonthermal background 
radio noise, which carries with 1 t the signature of the mean magnetic fields and the electron spectrum 1nvolved. Our problem therefore is to disentangle the information carried by the continuum radio emission by matching it suitably with the electron spectrum seen near the Earth and other astrophysical parameters. Atteinpts to connect the interstellar electron spectrum and the cosmic radto continuum through galactic magnetic flelds, have been made by many workers (Bierman and Davis, 1960; Sironi, 1965; Felten, 1966; Ramaty and Ligenfelter, 1966a; Okuda and Tanaka, 1968; Anand et a1, 1968a,b,c; Verma, 1968; Webber, 1968; Alexander et a1, 1970; Goldstein et a1, 1970; Stephens, 1971; Burger, 1971; Bulanov et a1, 1972; Cummings, et al, 1973b).

Since radio intensity is usually described in terms of the frequency of emission, one can rewrite Equation 5.1 as

$$
I(v)=\frac{1}{4 \pi} \int_{0}^{L(1, b)} e^{-f(\nu, s)} \psi(\nu, s) \text { ds Watt. } \mathrm{cm}^{-2} \mathrm{sr}^{-1} \mathrm{~Hz}^{-1}
$$

Here the emissivity function can be written as

$$
\psi(\nu, s)=1.04 \times 10^{-43} / B_{\perp}(s) G\left(\nu, E^{\prime}\right) j_{e}\left(E^{\prime}, s\right) \mathrm{dE}^{\prime} \text { Watt. } \mathrm{cm}^{-3} \mathrm{~Hz}^{-1} 5.13
$$

Where $B_{1}(s)$ is the perpendicular component of the magnetic field at $s$ expressed in Gauss; $j_{e}\left(E^{\prime}, s\right)$ is the cosmic ray electron intensity where $E^{\prime}$ is expressed in $\mathrm{GeV}$; and $G\left(\nu, E^{\prime}\right)$ is the power spectrum of the emitted radiation by an electron of energy $E^{\prime}$ (same as the function $F(x)$ defined by Ginzburg and Syrovatskil, 1965). The optical depth $f(\nu, s)$ can be written from Ginzburg and Syrovatskii (1965) as,

$$
f(v, s)=\frac{3.1 \times 10^{16}}{v^{2}} \int_{0}^{s} \Sigma_{i} \frac{n_{1}^{2} f_{1}^{2}(s)}{T_{f}^{3 / 2}}\left\{17.7+\ln \left(T_{i}^{3 / 2 / v)\}} k_{i} d s\right.\right.
$$

where $\mathrm{n}_{1}$ is the electron density at $\mathrm{z}=0 ; \mathrm{T}_{1}$ is the temperature of the medium; $f_{i}(s)$ is the distribution of the medium along the line of sight; and $k_{i}$ is the 
fraction of the volume occupled by the medium of the type $i$ (summation over 1 is needed when the medium is a complex one). We shall first summarize the observational data before we proceed to estimate the synchrotron spectrum using the above equation.

\subsubsection{Observational Data}

During the past decade or so, detailed and systematic radio surveys of the Galaxy have been carried out in a broad band of frequencies using wide angle and pencl1 beams. Making use of these surveys, radio spectra have been constructed for different galactic directions, of which the following are of interest to us: (i) the Anticentre, (i1) the Centre and (iii) the Halo.

\section{(i) The Anticentre}

The Anticentre has been defined here over a broad region corresponding to $l \approx 140-190^{\circ}$ and $\mathrm{b} \approx 10^{\circ} \mathrm{N}-10^{\circ} \mathrm{S}$. In order to deduce the radio spectrum, surveys with wide angle beams in the range 10-1407 $\mathrm{MHz}$ are used (Costain, 1960; Pauliny-Toth and Shakeshaft, 1962; Turtle et al, 1962; Parthasarathy and Lerfald, 1965; Andrew, 1966; Purton, 1966; Bridle, 1967; Howe11, 1970; Sironi, 1974; Webster, 1974). The radio spectrum thus derived for the Anticentre in the range $10-600 \mathrm{MHz}$ is shown in Figure 5.5 by Curve A. If the spectral form at any frequency is expressed as $I_{\nu} \propto \nu^{-\alpha}$, then it is evident from this figure that the spectral index $\alpha$ varles from about 0.3 at low frequencies increasing gradually to about 0.8 at about a few $100 \mathrm{MHz}$ and remains constant thereafter at least up to $1407 \mathrm{MHz}$ (Webster, 1974).

\section{(11) The Centre}

In this case two particular directions are chosen towards the Centre, but clearly avoiding the Nucleus; they are a) $1=0^{\circ}, \mathrm{b}=3.6^{\circ} \mathrm{N}$ and $3.6^{\circ} \mathrm{S}$ and b) $1=20^{\circ}$ and $340^{\circ} ; \mathrm{b}=0^{\circ}$. For the purpose of constructing the associated 


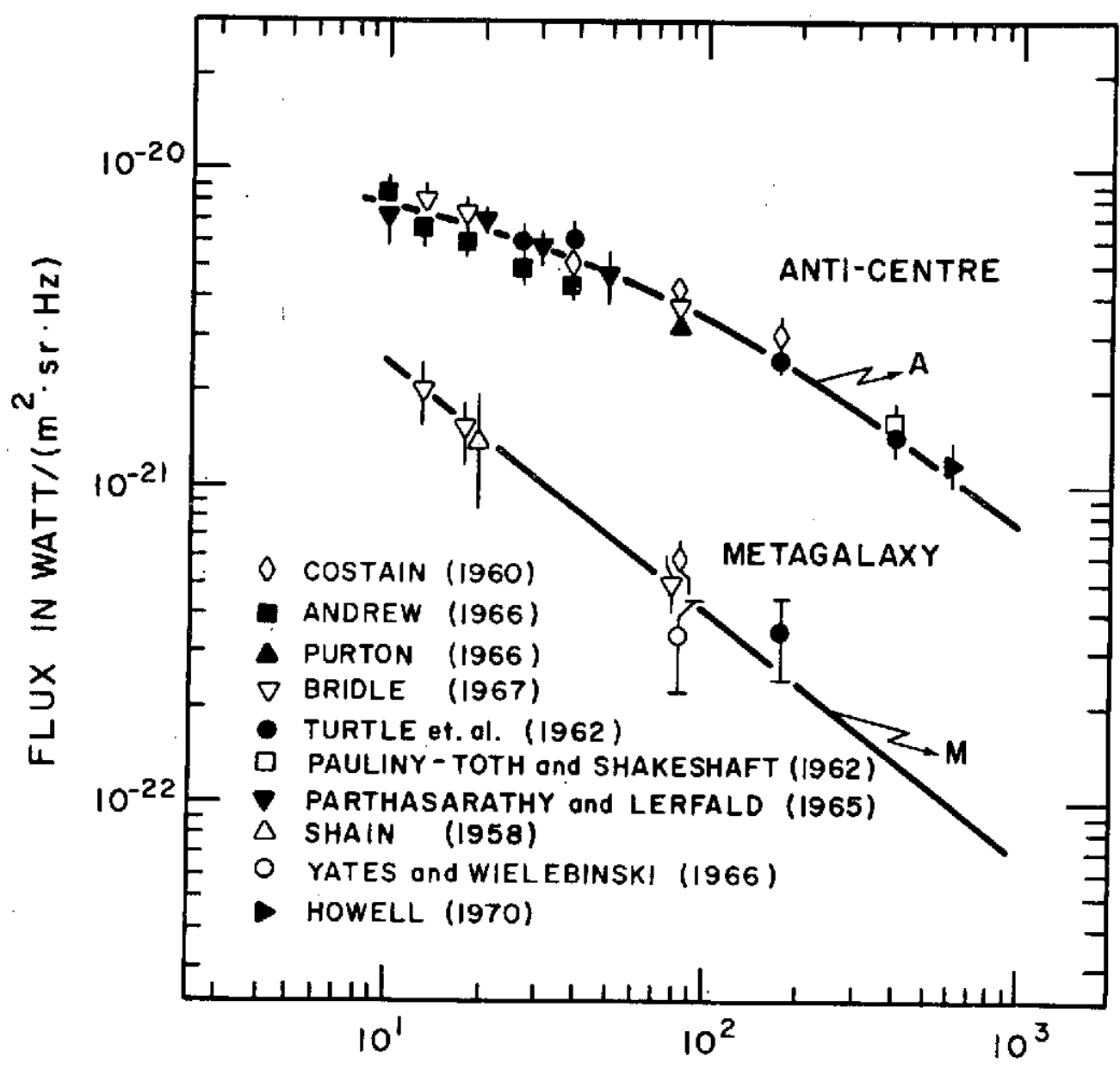

FREQUENCY IN $\mathrm{MHz}$

Fig. 5.5: Radio spectra in the direction of the Anti-centre (Curve A) and from the Matagalaxy (Curve $M$ ). 


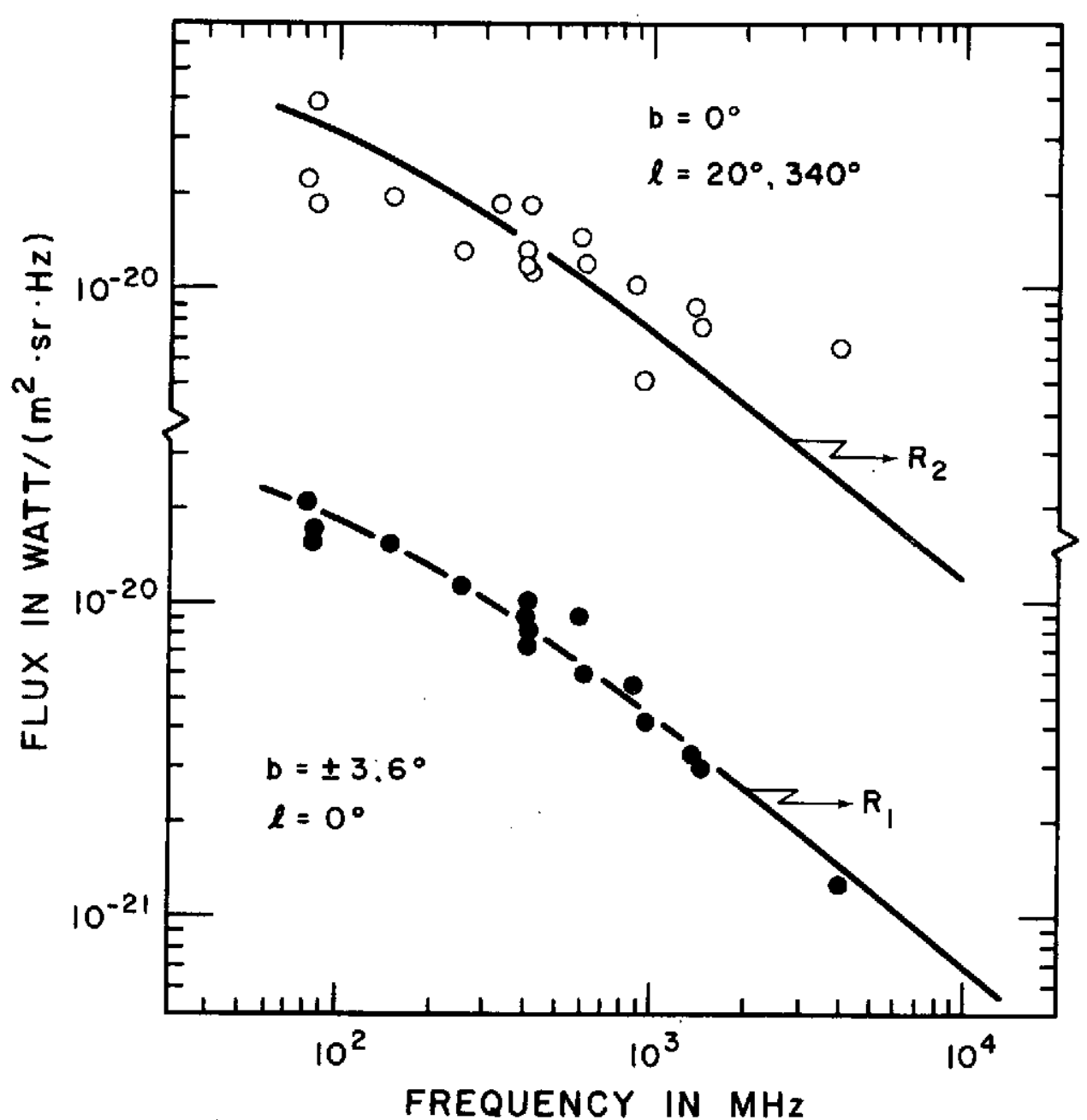

Fig. 5.6: Radio spectra in the general direction of the galactic centre but slightly displaced from the centre, in latitude (Curve $R_{1}$ ) and in longitude (Curve $R_{2}$ ); the references to data points are given In the text. 
rad10 spectrum, surveys made with pencil beams in the frequency range 80 $4080 \mathrm{MHz}$ are used (Baldwin, 1955a; Denisse et a1, 1955; Kraus and Ko, 1955; Plddington and Trent, 1956; H111 et a1, 1958; Westerhout, 1958; Seeger et al, 1960; W1Ison and Bolton, 1960; Large et al, 1961; Mathewson et al, 1962; Braccesi and Vespigani, 1964; Moron, 1965; Seeger et al, 1965; Komesaroff, 1966; Penzias and Wilson, 1966; Wielebinski et a1, 1968). These flux values shown in Figure 5.6 are the mean values in the two directions $b=3.6^{\circ} \mathrm{N}$ and $3.6^{\circ} \mathrm{S}$ in the case of $R_{1}$ and $1=20^{\circ}$ and $340^{\circ}$ for $R_{2}$, except in surveys, in which data is avallable for only one. It is apparent from this figure that the radio spectra $R_{1}$ and $R_{2}$ are similar in nature except that at frequencies greater than a $\mathrm{GHz}$, there seems to be a suggestion of a flattening of the spectrum in the direction $b=0$ which is presumably due to thermal radiations from the galactic plane.

\section{(i1i) The Halo}

The halo region is defined over a wide region of the sky away from the plane of the Galaxy. In the case of the North Halo, the regions centered around the halo minimum radiation $\left(1 \approx 190^{\circ}, \mathrm{b} \approx 50^{\circ}\right)$ have been chosen. Using the same surveys, as in the case of the Anticentre, the radio spectrum in this direction of the sky has been obtained at frequencies $\geq 10 \mathrm{MHz}$; at low frequencies, the works of Getmantsev et al. (1968) and Alexander et al (1969) have been used. These flux values plotted in Figure 5.7 show that below a few $\mathrm{MHz}$, the values decrease with decreasing frequency presumably as a result of absorption. A similar spectrum has been obtained towards the south galactic Pole (Yates and Wielebinski, 1966; Alexander et a1, 1969) and is shown in Figure 5.8. 


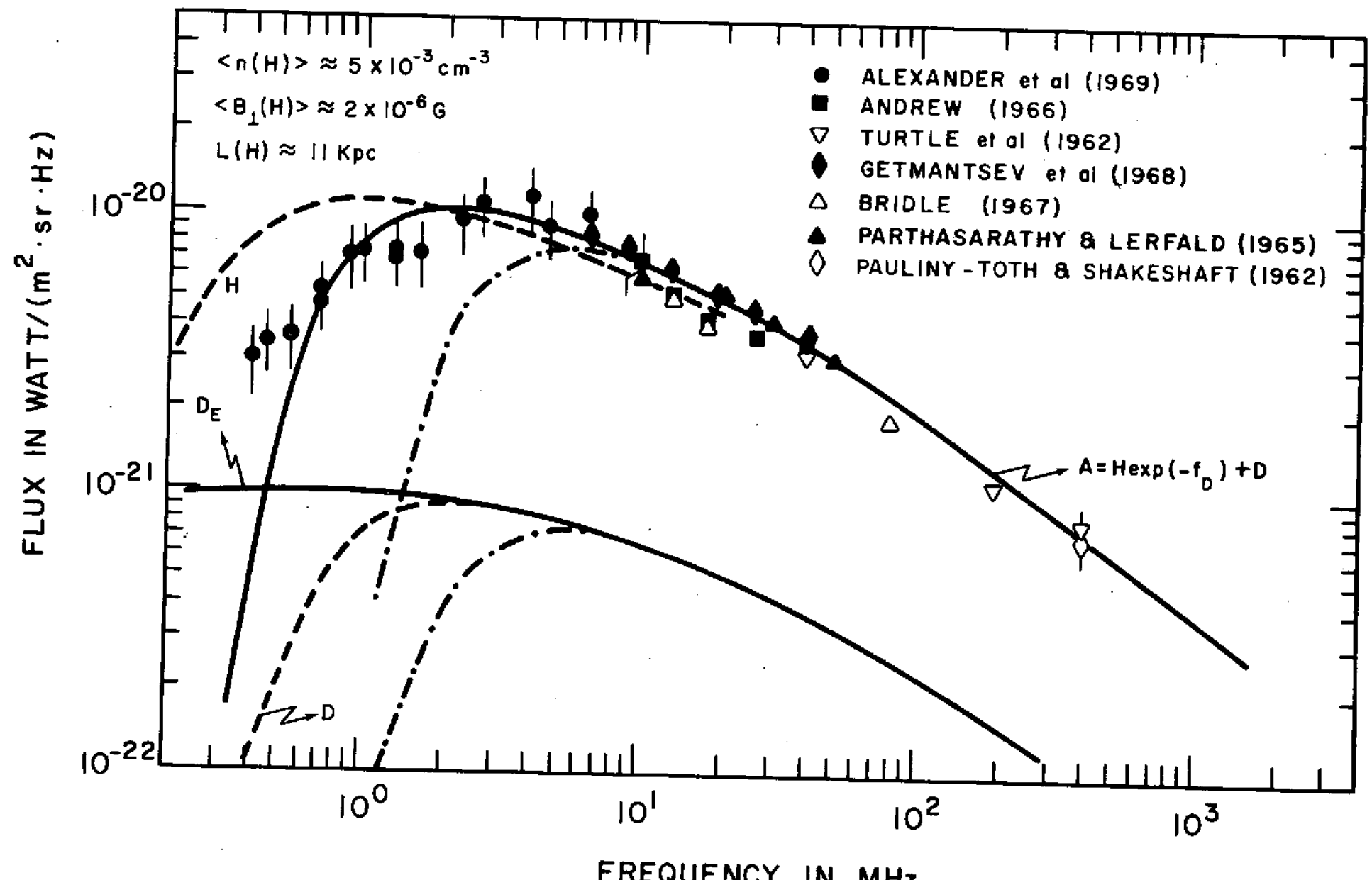

Fig. 5.7: Radio spectra in the direction of north halo minimum radiation. Curves $H$ and $D$ are respectively the spectra from the Halo and Disk after taking into account the self absorption, while $D_{E}$ is the emission spectrum in the Disk. The solid Curve A and the dash-dot curve are the expected spectra for two different models of interstellar gas. 


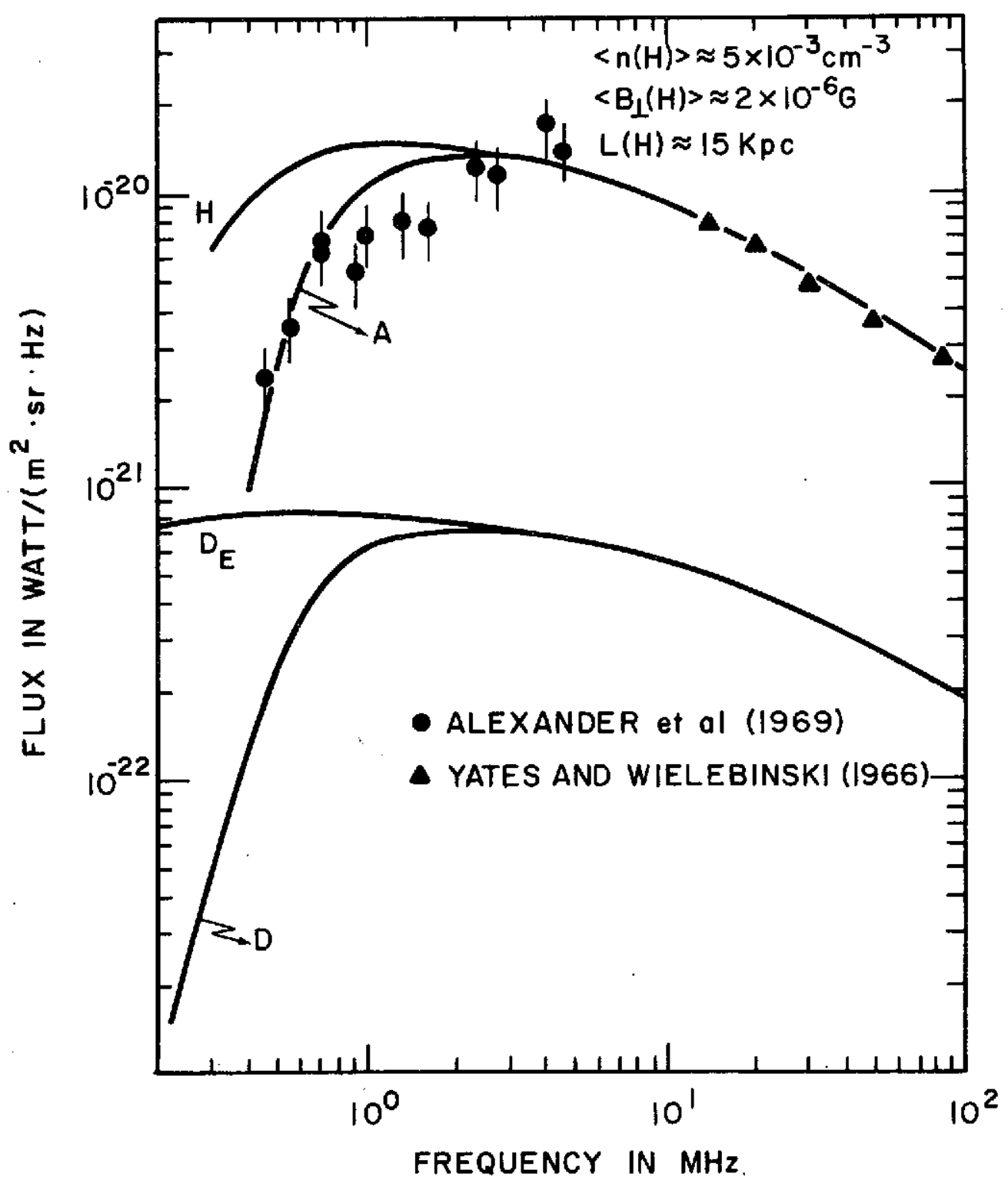

Fig. 5.8: Radio spectra towards the galactlc south pole. The curves shown in this figure are similar to those in Figure 5.7. 
It 18 imperative that before attempting to interpret the background radio emission as of galactic origin, one should enquire whether there could exist a substantial component of metagalactic origin in the observed radio intensities. Such attempts have been made (Sha1n, 1958; Turtle et al, 1962; Yates and W1elebinsk1, 1966; Purton, 1966; Bridle, 1967) and the information avallable is also included in Figure 5.5 as curve $M$. It can be seen that the metagalactic contribution at $20 \mathrm{MHz}$ is about $20 \%$ of the total radiation towards the Anticentre. Though there is some Indication that this spectrum is perhaps steeper than the galactic radiation, an attempt to detect its contribution at $2 \mathrm{MHz}$ revealed 1 ts near absence (Bridle, 1968). Since the latter observation indicates that the spectral shape of the metagalactic component has probably become flat at low frequencies, one can justiflably ignore its influence in the analysis of the galactic radiation (Daniel and Stephens, 1970; Stephens, 1971).

Now we can proceed to interpret the radio spectrum by combining it with our knowledge on cosmic ray electrons sampled near the Earth.

\subsubsection{The Energy Spectrum of Electrons in Interstellar Space}

Anand et al (1968a, b) first deduced a reliable and detailed energy spectrum of galactic electrons, as it exists in the near interstellar space by making use of the radio spectrum towards the Anticentre. In these calculations it was assumed that (a) the emissivity $\psi(\nu, s)$ is independent of $s$, and (b) the energy spectrum. of electrons observed near the Earth above $5 \mathrm{GeV}$ is well preserved in the near interstellar space; also single values for the mean magnetic field $\left\langle\mathrm{B}_{1}\right\rangle$ and the cosmic ray density $\rho_{\mathrm{C} . \mathrm{R}}=1$ were assumed along the line of sight. Under these assumptions Equation 5.12 is evaluated through Equation 5.13 by taking an effective path length of $4 \mathrm{kpc}$ towards 
the Anticentre. It can be shown that, since the second assumption requires an interstellar electron spectrum below $5 \mathrm{GeV}$, which presumably should smoothly foin the observed spectrum above $5 \mathrm{GeV}$, it is possible to assign a single value for $\left\langle\mathrm{B}_{1}\right\rangle$ and a unique smoothly varying electron spectrum to match the radio spectrum. The electron spectrum thus deduced for the Anticentre is shown as the radio emitting electron spectrum by curve A in Figure 4.19; the corresponding value of $\left\langle\mathrm{B}_{1}\right\rangle$ is $5 \mu \mathrm{G}$. The uncertainty in the deduced electron spectrum increases with decreasing energy and is about a factor 2 around $300 \mathrm{MeV}$ increasing to very large value below $100 \mathrm{MeV}$ (Cumnings et al, 1973b), due to the effect of interstellar absorption which dominates below a few MHz. Nevertheless, we feel that the smooth spectrum shown by Curve A in Figure 4.19 could be the most probable electron spectrum in interstellar space because it is quite obvious from Figures $5.5,5.7$ and 5.8 that the radio spectral index changes gradually from 0.8 to. 0.3 in the frequency range 200-10 MHz, where there is no absorption effect.

Having derived the electron spectrum towards the anticentre region, it is natural to enquire whether this spectrum could also explain the observations in the other galactic directions. In the direction of the Centre, it is found that the same electron spectrum could reproduce the observed radio spectra (curves $R_{1}$ and $R_{2}$ of Figure 5.6) with a single value of $\left\langle B_{1}\right\rangle=$ 7.2 $\mu \mathrm{G}$ for the line of sight $\mathrm{L}_{\mathrm{R}_{1}}=13 \mathrm{kpc}$ and $\mathrm{L}_{\mathrm{R}_{2}}=22 \mathrm{kpc}$. In the same manner, the halo spectra can also be well explained above a few MHz with a magnetic field $\left\langle\mathrm{B}_{1}\right\rangle=2 \mu \mathrm{G}$ for $\mathrm{L}_{\mathrm{H}}=11 \mathrm{kpc}$ towards the north halo minimum (Figure 5.7) and $I_{H}=15 \mathrm{kpc}$ towards the south Pole (Figure 5.8). From this, one may infer that the spectral shape of cosmic ray electrons remains the same in all regions of the Galaxy. 


\section{(ii) Magnetic Fields in the Galaxy}

We have noted already that if $\left\langle\rho_{C . R}\right\rangle=1$ applies to all regions in the Galaxy, then one can obtain a unique value for the mean perpendicular component of the magnetic field along any galactic direction from an analysis of the radio continuum in the Galaxy. Such values of $\left\langle B_{1}\right\rangle$ in the direction of the Anticentre and Centre in the Galaxy are about 5 and $7 \mu G$ respectively. This variation in the magnetic field strength can efther be real or be a reflection of the effect due to $\rho_{C} . R$ being $\neq 1$ at all regions, combined with a possible varlation of the magnetic field. If one assumes random field orientations, the above results indicate that the total magnetic fleld varies from about $6 \mu \mathrm{G}$ to about $9 \mu \mathrm{G}$ in the Disk. As for the Halo, the derived magnetic field has a value of about $2.5 \mu \mathrm{G}$; the uncertainty in this value depends upon the value of $\rho_{C . R}$ and the extent of the cosmic ray halo. It may be noted that the magnetic field strength deduced for the Disk here seems to be much larger than the value of about $3 \mu G$, commonly used by astrophysictsts. In order to resolve this difference, the following explanation has been advanced by Cowsik and Mitteldorf (1974). The fluctuations in the magnetic field strength, whose magnitude is found to be of the order of the average field itself $\langle\Delta B\rangle_{\text {rns }} \sim B_{0}$ (Jok1pil and Lerche, 1969), can arise from the compression and rarefaction of the ambient field by gas motions, which are coupled to the lines of force in interstellar space. This process can be accompanied by a correlated variation in the density of cosmic ray electrons due to betatron process. As a consequence, the synchrotron emissivity varies non-linearly (Equation 5.13) resulting in an enhanced mean emissivity depending upon the value of $\langle\Delta B\rangle_{\text {rms }}$; this could be as large as a factor $\sim 10$ for $\langle\Delta B\rangle_{\text {rms }}$ $\approx \mathrm{B}_{\mathrm{O}}$. 


\subsubsection{Mean Density of Cosmic Rays in Different Regions of the Galaxy}

It has been demonstrated above that a consistent picture can be constructed to explain satisfactorily the observed non-thermal radiation from different regions of the Galaxy, including the Halo. At the same time it Is instructive to examine how much variations can be permitted in the value of ${ }^{P_{C} . R}$ used. One way of doing this is to assume the same value of $\left\langle B_{1}\right\rangle$ deduced for the anticentre direction to apply for the direction of the galactic centre and then to estimate the value of $<\rho_{\mathrm{CR}}>$ for the latter direction. If one does this 1 t leads to a mean cosmic ray density $\left\langle\rho_{C . R}>\approx 1.8\right.$ towards the Centre. Thus one finds that the cosmic ray density averaged over the direction as the Centre is unlikely to exceed by very much more than a factor of 2 in comparison with the outer regions. It has also been shown by Anand et al (1968b) that the cosmic ray density in the Halo cannot be lower than in the Disk by a factor as much as 2 or more. However, if cosmic rays are confined to the Disk and they slowly leak into the halo region, where the mean free path could be much larger than in the Disk, the density of cosmic rays in the Halo has to be very much smaller than in the Disk (Laster, 1964). This apparent contradiction arises from the fact that we have assumed a uniform distribution of cosmic rays. Since this is a simple minded picture one should keep an open mind for possible large scale variations of the cosmic ray intensity in localized regions as well as a possible general gradient from the Nucleus towards the Poles or to the periphery of the Disk.

\subsubsection{Some Inferences on the Properties of Interstellar Medium}

Since the optical depth depends upon the physical properties of the interstellar medium, it has been pointed out (Stephens, 1971) that the observed radio spectrum in the direction of the Halo, and the electron spectrum derived earlier, can be used to distinguish between various models of interstellar medium. In Figure 5.7 , the estimated emission spectrum from 
the Disk towards the north halo minfmum is shown by Curve $D_{E}$ for a mode1 in which cold Interstellar clouds are postulated to be immersed in the hot intercloud medium. Curve $D$ in this figure is the expected spectrum when absorption is taken Into account by setting in Equation 5.14 for the intercloud medium the values $\mathrm{n}_{1}=0.04 \mathrm{~cm}^{-3}, \mathrm{~T}_{1}=9000^{\circ} \mathrm{K}, \mathrm{k}_{1}=1$ and $\mathrm{f}_{1}(\mathrm{~s}$ ) $=\exp \left(-\mathrm{s}^{2} \sin ^{2} \mathrm{~b} / 2 \sigma^{2}\right)$, where $\sigma_{1}=160 \mathrm{pc} ;$ for the clouds one has $\mathrm{n}_{2}=0.02$ $\mathrm{cm}^{-3}, \mathrm{~T}_{2}=90^{\circ} \mathrm{K}, \mathrm{k}_{2}=0.02$ and $\mathrm{f}_{2}(\mathrm{~s})=\exp \left(-\mathrm{s}^{2} \sin ^{2} \mathrm{~b} / 2 \sigma_{2}^{2}\right)$, where $\sigma_{2}=120 \mathrm{pc}$; these parameters are similar to those derived by Field et al (1969) from other consideration. Curve $H$ is the radio spectrum from the Halo after taking into account the absorption in the Halo by setting in Equation 5.14, $\mathrm{n}=0.005 \mathrm{~cm}^{-3}, \mathrm{~T}=10^{4}{ }^{\circ} \mathrm{K}, \mathrm{f}(\mathrm{s})=\mathrm{k}=1$. Curve A in the same figure is the sum of the radiations calculated for the Disk (Curve D) and for the Halo after taking into account the absorption of halo radiation In the Disk. The dash-dot curves are the calculated spectra for another set of parameters with $\mathrm{T}_{1}=1000^{\circ} \mathrm{K}, \mathrm{T}_{2}=50^{\circ} \mathrm{K}$ and $\mathrm{n}_{2}=0.03 \mathrm{~cm}^{-3}$, the other parameters remaining the same, as deduced by Hjellming, Gordon and Gordon (1969). One finds that while curve $A$ is in good agreement, the dash-dot curve is at variance with the observations. It is also found (Stephens, 1971) that even in the absence of clouds, the fit obtained by curve A demands a temperature $\mathrm{T}_{1}>4000^{\circ} \mathrm{K}$, thereby ruling out the possibility that the temperature of the intercloud medium could be as $10 \mathrm{w}$ as $1000^{\circ} \mathrm{K}$. A similar estimate of the radio spectrum towards the south Pole using the first set of parameters confirms the earlier deductions as one can see from Figure 5.8.

\subsubsection{The Galactic Halo}

The existence of a galactic halo of near spherical shape, glowing in 
the radio region by synchrotron radiation emitted by relativistic electrons spiralling in weak magnetic flelds existing therein, was first postulated by Shklovsky (1952). This suggestion was made credtble by Baldwin (1955b), who on the basts of cosmic background radio observations, attributed a diameter of $20-30 \mathrm{kpc}$ for this halo. The existence of the radto halo, which received almost universal support from observational radio astronomers, astrophysicists, and cosmic ray physiclsts for over a decade, and was sometimes acclaimed as one of the most important discoveries of our times, is now being increasingly questioned during recent years. The arguments so far advanced against its existence have been drawn from recent observations on the galactic background radio emission which show structural features. If one considers that these structural features, as revealed by pencil beam surveys are localized phenomena within the Disk and that the general background is indeed composed of the integrated emission from such features, then there can be no evidence for the existence of the radio halo. However, this extreme hypothesis can be understood only if the general magnetic fleld in the galaxy is exceedingly small $\left(\ll 10^{-6}\right.$ gauss) and that the emission takes place essentially in discrete regions where the magnetic field ( $>10^{-5}$ gauss) and the cosmic ray density are very high; this however seems very difficult to accept from the study of optical polarization (Mathewson and Nichols, 1968). Granting then that the radio emissivity can be considered to be nearly uniform in the Disk, which seems reasonable, it has been shown (Anand et al, 1968c; Daniel and Stephens, 1970) that the present observational evidence cannot rule out the existence of a radio Halo from the following arguments; (i) It is evident from Figures 5.5 and 5.7 that the combined contribution from the Disk and the Metagalaxy towards the minimum halo direction may not 
exceed $50 \%$ of the total radiation observed in that direction; the excess radiation should therefore have its origin from the Halo; (ii) It can also be shown that the ratios of the excess radiation towards different directions in the Halo to that towards the minimum radiation are consistent with a volume much thicker than the Disk. Thus one is faced with the situation that the cosmic ray observations suggest either no Halo or a leaky halo, while the radio observation cannot at present rule out the existence of a radio halo.

6. The Role of Cosmic Rays in Galactic Dynamics:

We have so far considered in detail problems relating to the propagation of cosmic rays in galactic space and the many consequences arising from the particulate nature of this radiation. At the same time one recognizes that cosmic rays in bulk constitute a relativistic gas with its own contributory pressure and energy density in galactic space, comparable to those due to magnetic fields and thermal motion of gas in interstellar space. Though these facts were generally known for a long time, it is only during recent years their real signiflcance has been increasingly realized resulting in a quest to investigate the consequences of the extstence of the cosmic ray gas in galactic space.

The role that cosmic rays play in galactic dynamics may be briefly stated as follows. The interstellar gas is confined to the Disk by gravitational potential resulting from the distribution of stars perpendicular to the plane of the Galaxy; in this the gas has its own random motions. At the same time, the magnetic fleld is tied to the gas because it is partly Ionized; cosmic rays in turn are tied to the magnetic fleld lines. A11 these forms of energy exert pressure against the gravitational force. Hence, 
cosmic rays play an important role in the hydrostatic equilibrium and gravitational stability of gas in the Disk. Further, the subrelativistic cosmic rays, during their propagation, lose their energy rapidly through ionization before they are thermalized; as a consequence they heat and Ionize the interstellar gas. The stability and thermal phase of the gas then depend on the density of low energy cosmic rays existing in interstellar space. In the ensuing sections, we present some of the basic approaches that have been tried so far to demonstrate these effects, and the reader is cautioned that no attempt has been made to review this subject.

\subsection{Hydrostatic Equilibrium of the Gaseous Component of the Galaxy:}

The thickness of the Disk, composed mainly of stars, is maintained by the gravttational acceleration $\mathrm{g}(z)$, resulting from the distribution of stars and gas, against the random motion of stars and can be described by the hydrostatic equilibrium equation

$$
<v_{S}^{2}(z)>\frac{d}{d z} \ln \rho_{s}(z)=\frac{d \phi(z)}{d z}
$$

where $\left\langle v_{S}^{2}(z)\right\rangle^{\frac{1}{2}}$ is the root-mean square of the random velocities of stars, which is about $18 \mathrm{~km} . \mathrm{sec}^{-1}$ in the neighborhood of the Sun (Wooley, 1965), $p_{S}(z)$ is the star denstty and $\phi(z)$ is the gravitational potential. The two dimensions in the plane of the Disk, in which the centrifugal force of rotation of the Galaxy is balanced by the gravitational attraction of the Nucleus, are not directly relevant here and hence the hydrostatic equation relating to the plane may be written as (Parker, 1969)

$$
\frac{d}{d z}\left\{p(z)+B^{2}(z) / 8 \pi+P(z)\right\}=\rho(z) g(z)
$$


Here, $p(z)=\left\langle v^{2}(z)\right\rangle \rho(z)$ is the gas pressure, $B(z)$ is the magnetic field strength and $P(z)$ is the cosmlc ray pressure, which is one third of 1 ts energy density.

In the above equations, it is implicitly assumed that the external pressures due to magnetic field and cosmic rays existing outside the Disk and the weight of halo gas are negligible. If we make a further assumption that the distribution of magnetic field strength and cosmic ray density in the $z$-plane is similar to that of gas namely, $B^{2}(z) / p(z)=B^{2}(0) / p(0)$ and $P(z) / \rho(z)=P(0) / \rho(0)$, then Equation 6.2 reduces to

$$
Q^{2} \frac{d}{d z}\{\ln \rho(z)\}=g(z)
$$

where $Q^{2}=\left\langle v^{2}(z)\right\rangle+B^{2}(0) /\{8 \pi \rho(0)\}+P(0) / \rho(0)$. This equation can be integrated to get the distribution of gas as

$$
\rho(z)=\rho(0) \exp \left\{\frac{1}{Q^{2}} \int_{0}^{z} g(z) d z\right\}
$$

The acceleration parameter in this equation can be determined as a function of $\mathrm{z}$ from an analysts of star counts and radial velocities of K-giants using Equation 6.1; according to Oort (1960) the values of $|g(z)|$ at $z=0,100$, $200,300,400$ and $500 \mathrm{pc}$ are respective1y $0.0,2.5,4.3,5.46 .2$ and $6.8 \mathrm{x}$ $10^{-9} \mathrm{~cm} . \mathrm{sec}^{-1}$. In Figure 6.1 is shown the distribution of gas $\rho(z) / \rho(0)$ as a function of $z$. The dashed curve is the experimentally determined distribution by Schmidt (1957) corrected for the galactic scale Ro $=10 \mathrm{kpc}$ (Section 2.1). The solid line is the calculated curve using Equation 6.4 (Ke11man, 1972a) for $Q=9.84 \mathrm{~km} \cdot \mathrm{sec}^{-1}$. One can see the good agreement between the calculated and observed distributions within about $250 \mathrm{pc}$ and one obtains

$$
\rho(0)<v^{2}(z)>+B^{2}(0) / 8 \pi+P(0)=9.7 \times 10^{11} \rho(0)
$$




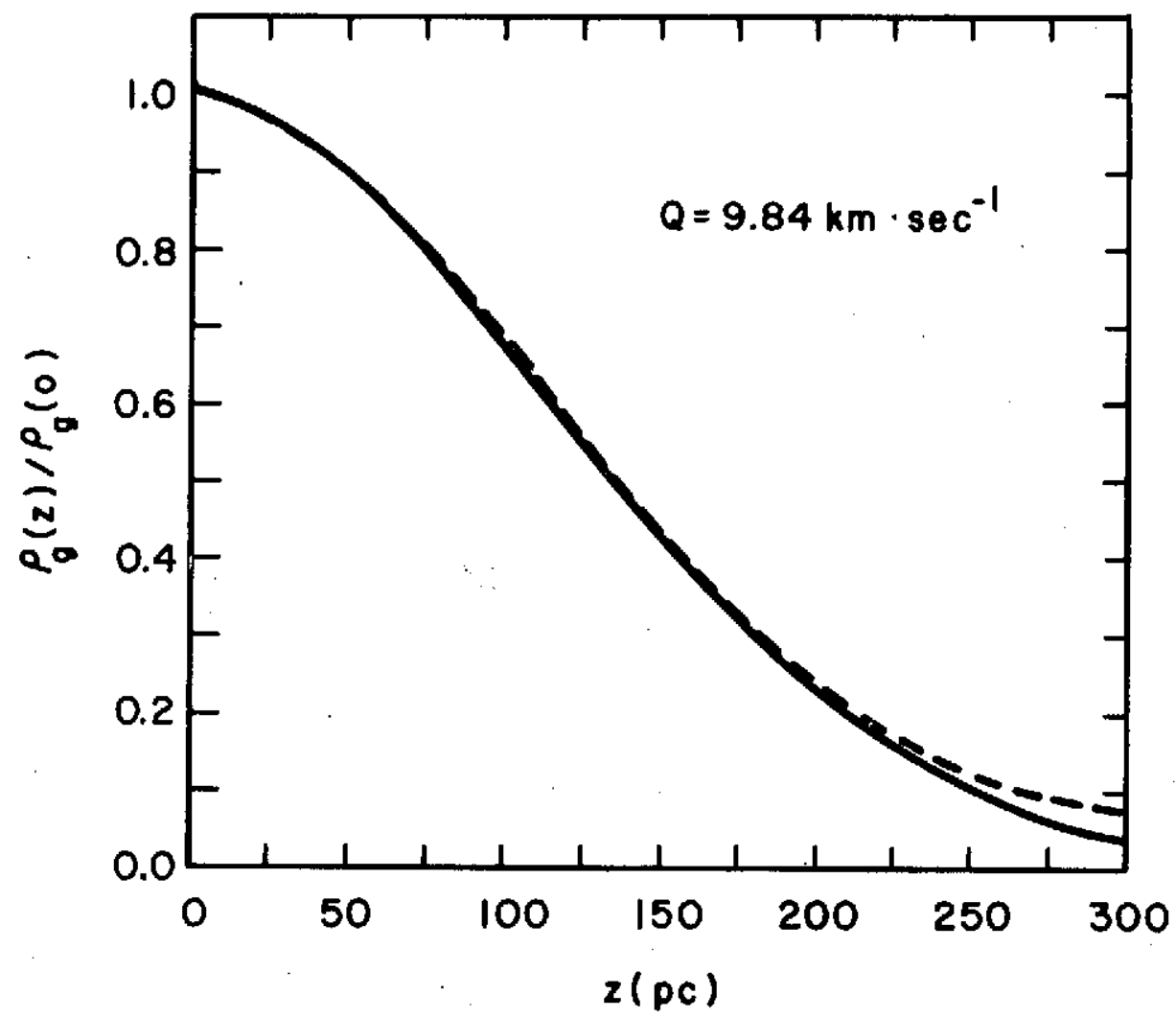

Fig. 6.1: The observed distribution of gas perpendicular to the galactic plane (dotted curve) is compared with the calculated one (solid curve). 
From the above equation it is possible to derive the value for any one parameter, if one knows the other two. However, in practice one notes that at present, all the three quantities are known with comparable degrees of uncertainty with the result that there is no spectal merit in deducing the value of one by using the other two. Nonetheless, to get a feel for the magnitude of these quantities one can assume that they contribute equally and thereby deduce the following values: $\left\langle v^{2}\right\rangle^{\frac{1}{2}} \approx 5.7 \mathrm{~km} \cdot \mathrm{s}^{-1}, \mathrm{P} \approx 5.2$ dynes. $\mathrm{cm}^{-2}$ and $B \approx 3.6 \mu \mathrm{G}$ for $\rho(0)=1$ hydrogen atom. $\mathrm{cm}^{-3}$. These numbers can be now compared with those available from observations.

From a study of the velocity profiles of $21 \mathrm{~cm}$ hydrogen line, it is found that the velocity dispersion for clouds (Westerhout, 1957) as wel1 as for intercloud medium (Heiles, 1967) is about $6 \mathrm{~km} \cdot \mathrm{s}^{-1}$; this is in good agreement with the value deduced above. In case of cosmic ray pressure, though the equipartition value is consistent with that observed in the nelghborhood of the Earth, one suspects that the true pressure in interstellar space is 1ikely to be much larger. Similarly, the mean magnetic field obtained from the radio brightness distribution (Section 5.3.3) is also larger than the value deduced above. This could imply that the internal pressure might be numerically larger than the gravitational force to achieve hydrostatic equilibrium. On the other hand in our assumptions, we have neglected the external pressures, such as those due to the presence of gas, cosmic rays and magnetic field in the halo region, which could in principle compensate this apparent higher internal pressure. One also notices from F1gure 6.1 that the theoret1cal curve deviates from the observed one at large values of $z$. This again is due to the assumption that the distribution of $\rho, P$ and $B$ are identical, which is not true because we know that the radio 
disk is much thicker than the gas disk (Section 2.1). Hence Equation 6.2 needs to be solved more rigorously and the final fit to the observed data would give information on the possible distribution of cosmic rays and magnetic fields away from the galactic plane.

\subsection{Stability of Self Gravitating Gas and the Formation of Clouds}

The condition for the stablity of self-gravitating gas can be understood from the following concept (Jean, 1928). Let us consider a uniform distribution of gas, in which a small condensation at a point $P$ is accompanied by a compensating rarefaction at a nearby point $Q$. This disturbance results in a gain in the total internal energy, which then travels about with the velocity of sound until it is dissipated by viscosity in the form of heat energy. Such a process gives rise on one hand, to an increase in the thermodynamical energy of the gas but on the other, it causes

a decrease in the gravitational energy. If $P$ and $Q$ are sufficiently far apart, the decrease in the gravitational energy becomes numerically greater than the increase in the thermodynamical energy and as a result the medium acquires kinetic energy at no expense; this will continue to grow indefinitely. Th1s form of instability resulting through displacements in which condensation and rarefaction occur in pairs at sufficiently distant points in the medium is called the "Jeans gravitational instability".

For a uniform gas with a uniform gravitational potential throughout, Jeans (1928) has showed that the hydrodynamical equation relating to the gas motions described above can be written as

$$
\frac{\mathrm{d}^{2} \mathrm{~s}:}{\mathrm{dt^{2 }}}=4 \pi \mathrm{G \rho} s+\mathrm{C}^{2} \nabla^{2} \mathrm{~s}
$$

where $\mathrm{s}$ is the condensation parameter $\delta \rho / \rho, \mathrm{C}$ is the speed of sound in the medium and $G$ is the gravitational constant. Considering now a pure wave 
motion along the $X-a x i s$, with $s$ being proportional to $\operatorname{Cos}(2 \pi x / \lambda)$, the above equation becomes

$$
\frac{\mathrm{d}^{2} \mathrm{~s}}{\mathrm{dt^{2 }}}=\left[4 \pi \mathrm{G} \rho-\left(\frac{2 \pi}{\lambda}\right)^{2} \mathrm{c}^{2}\right] \mathrm{s}
$$

where $\lambda$ is the wavelength. The solution to Equation 6.7 , in which $s$ is proportional to $\exp ( \pm i q t)$, represents the wave motion, where $q^{2}=(2 \pi / \lambda)^{2} C^{2}$ - $4 \pi \rho G$, with a velocity of propagation

$$
v=\left[C^{2}-(\lambda / 2 \pi)^{2} 4 \pi G \rho\right]^{\frac{1}{2}}
$$

One can see from this equation that, when the gravitational term is omitted, the wave motion which travels with a uniform velocity $\mathrm{C}$ is independent of $\lambda$. The restoration of the gravitational term decreases the velocity of wave propagation, and since this term is proportional to $\lambda^{2}$; it is ineffective for short wave lengths. As we slowly increase the value of $\lambda$, Equation 6.8 becomes zero and then becomes imaginary. For such values of $\lambda$ there can not be any normal propagation of waves and the inftial condensation and rarefaction will increase exponentfally leading to unstable motion. The value of $\lambda$ for marginal instability, $i . e$. when the wave motion ceases to exist (according to Equation 6.8) is $=c \sqrt{\pi} / G \rho$. The radius of gas $R_{J}$ under marginal instability is $\lambda / 2$ which is about $0.4 \mathrm{kpc}$ for $\left\langle\mathrm{v}^{2}\right\rangle^{\frac{1}{2}} \approx 5 \mathrm{~km}$. $\sec ^{-1}$ and $\rho \approx 1$ hydrogen atom. $\mathrm{cm}^{-3}$. Thus, under simple Jeans' instability the interstellar gas will start forming clouds in scale sizes larger than $0.4 \mathrm{kpc}$ in radius. The time scale for the onset of instabtlity to the formation of clouds is about $\mathrm{q}^{-1} \sim 10^{8} \mathrm{yrs}$.

The effect of the inclusion of magnetic fields and cosmic rays in the self gravitating gas is to increase the internal energy of the system. From 
an analysis of hydrodynamic equations relating to this gas-field system, it has been shown by Kellman (1972b) that the radius of marginally unstable gas increases by a factor $(1+\alpha+\beta)^{\frac{2}{2}}$, where $\alpha$ and $\beta$ are the ratios of magnetic pressure to gas pressure, and cosmlc ray pressure to gas pressure respectively. When these parameters are comparable, the instability starts at wavelengths corresponding to $R=\sqrt{3} R_{J}$. It has been further shown by Ledoux (1951) that for plane parallel, non-rotating gas layer such as the interstellar gas, the radius of marginally unstable gas at the plane of symmetry is larger by an additional factor $\sqrt{2}$. Thus, for interstellar gas the value of $R \approx \sqrt{6} R_{J}$.

The above treatment shows that the self gravitating gas tends to be stable against the simple gravitational instability under the influence of magnetic field and cosmic rays which add to the internal pressure. This situation is true for the interstellar gas only at the plane of symmetry at $z=0$, where the magnetic field is paralle1 to the plane and is unfform. However, it was pointed out earlier (Section 3.2.3) that the instability in the gas field system inflates the magnetic field lines and the gas tends to slide along the field lines under the gravitational force. In Figure 6.2 is shown a schematic diagram of an idealized vertical section through the Disk, taken along the lines of force (Parker 1968b). When the lines of force are perturbed as shown by the sinusoldal line, the gas tends to slide down into the troughs along the field. This burdens the low regions causing them to sink further and unburdens the high places permitting them to expand upward. Similarly, the cosmic ray pressure is lower than the ambient pressure at high places contributing to the inflation and expansion of the field. The following simple treatment by Parker (1969) illustrates this process clearly. 


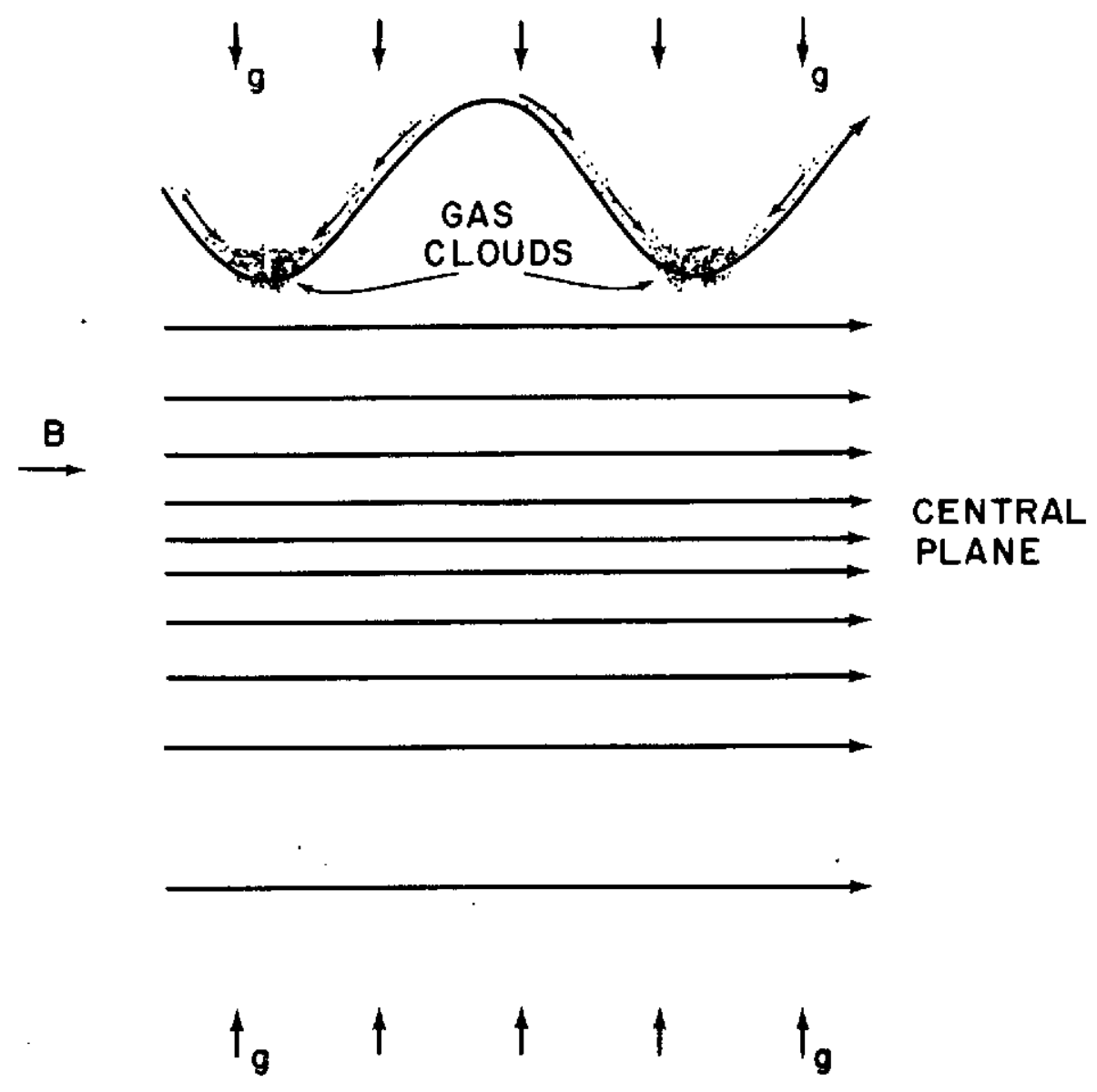

Fig. 6.2: A schematic drawing of the gas field system perpendicular to the plane of the Galaxy. 
Let the magnetic field $\underline{B}$ be along the $y$-direction and the gravitational acceleration be in the negative $z$-direction. Consider two elements of mass $m_{1}$ and $m_{2}$ suspended on a line of force. The welght $m_{1}$ distorts the field and changes the field from $(0, B, 0)$ to $\left(\Delta B_{x}, B+\Delta B_{y}, \Delta B_{z}\right)$ at the position of $\mathrm{m}_{2}$; the slope of the lines of force through $\mathrm{m}_{2}$ is approximately $\Delta \mathrm{B}_{\mathrm{z}} / \mathrm{B}$. As a result $\mathrm{m}_{2}$ is pushed towards the negative direction of $\mathrm{y}$ along the magnet1c field with a force $m_{2} g \Delta B_{z} / B$. In case of the magnetic field being pervaded by a tenuous background plasma with negligtble pressure and weight, the distorsion of field, at a remote position $(x, y, z)$ due to mass $m_{1}$ at the origin, exert a force on $\mathrm{m}_{2}$ given by

$$
F(r)=-m_{1} m_{2} g^{2}(z) y\left[1+\left(y^{2}+z^{2}\right) /\left(x^{2}+z^{2}\right)-2 x^{2} r^{2} /\left(x^{2}+z^{2}\right)^{2}\right] B^{2} x^{3} \quad 6.9
$$

where $r^{2}=x^{2}+y^{2}+z^{2}$. This can be compared with the force due to direct acceleration between $m_{1}$ and $m_{2}$, namely $F_{G}(x)=G m_{1} m_{2} y / r^{3}$. The first term In Equation 6.9 , which is zero at the plane of symmetry where $g(0)=0$, increases numerically with $|z|$ and exceeds very much larger than the simple self gravitating force. Hence, the total effect on mass $m_{2}$ is proportional to $G=\mathrm{g}^{2} / \mathrm{B}^{2}$ instead of $\mathrm{G}$. Parker has further shown that the time scale between the onset of instability and the formation of clouds is $(\Lambda / g)^{\frac{3}{2}} \sim 10^{7}$ yrs, where $\Lambda$ is the scale height of the gas in the z-direction; this value is very much smaller than for the simple Jeans' instability.

One can see from Equation 6.9 that, while the second and third terms average to zero over any plane $\mathrm{y}=$ constant, they are significant for large values of $y$. In the limit of large $y$, Equation 6.9 reduces to

$$
F(r)=-m_{1} m_{2} g^{2}(z)\left[\left(z^{2}-x^{2}\right) /\left(z^{2}+x^{2}\right)^{2}\right] / B^{2} \quad 6.10
$$

This force is attractive when $z>x$. The gas, which is displaced in $z$ below or above a cloud is powerfully attracted towards the cloud while those displaced in $x$ are repelled. In this picture, the initial instabilities may 
grow over scales of the order of $100 \mathrm{pc}$ along the lines of force and 10 to $100 \mathrm{pc}$ across the lines of force. Further, the state of motion of gas clumps would continue unt1l the gas density becomes suffictently large for self gravitation to take over the repulsive force described above and the internal forces resulting from thermodynamical, magnetic and cosmic ray pressures.

\subsection{Thermal Equilibrium of Interste1lar Gas:}

The observed physical properties of interstellar gas have been interpreted (Section 2.3) as due to the gas being composed of cool, dense clouds embedded in a hot, rarefied intercloud medium. In this context, Hayakawa et al (1961) were the first to suggest that low energy cosmic rays are quite effective in heating the interstellar medium. The heating can proceed through primary Ionization of gas atoms by the direct interaction of low energy cosmic rays and through secondary ionization by electrons liberated in the primary ionization process. The cooling of gas, which is very essential to maintain thermal equilibrium, is mainly through the de-excitation of atoms, both ionized and neutra1, and free-bound and free-free emission of free electrons. The evaluation of the equilibrium phase of the gas is a complex mathematical exercise (Field, 1970; Dalgarno and McCray, 1972) and hence in this section we only indicate the results from one such calculation, and summarize the various processes through which cosmic rays could contribute to the heating of interstellar gas.

It is quite obvious that for a given rate of heating, the equilibrium free electron density and the temperature of the medium depend upon the density of the gas. In Figure 6.3, we show the result from a typical calculation (Field et a1, 1969), in which the equilibrium temperature is plotted 


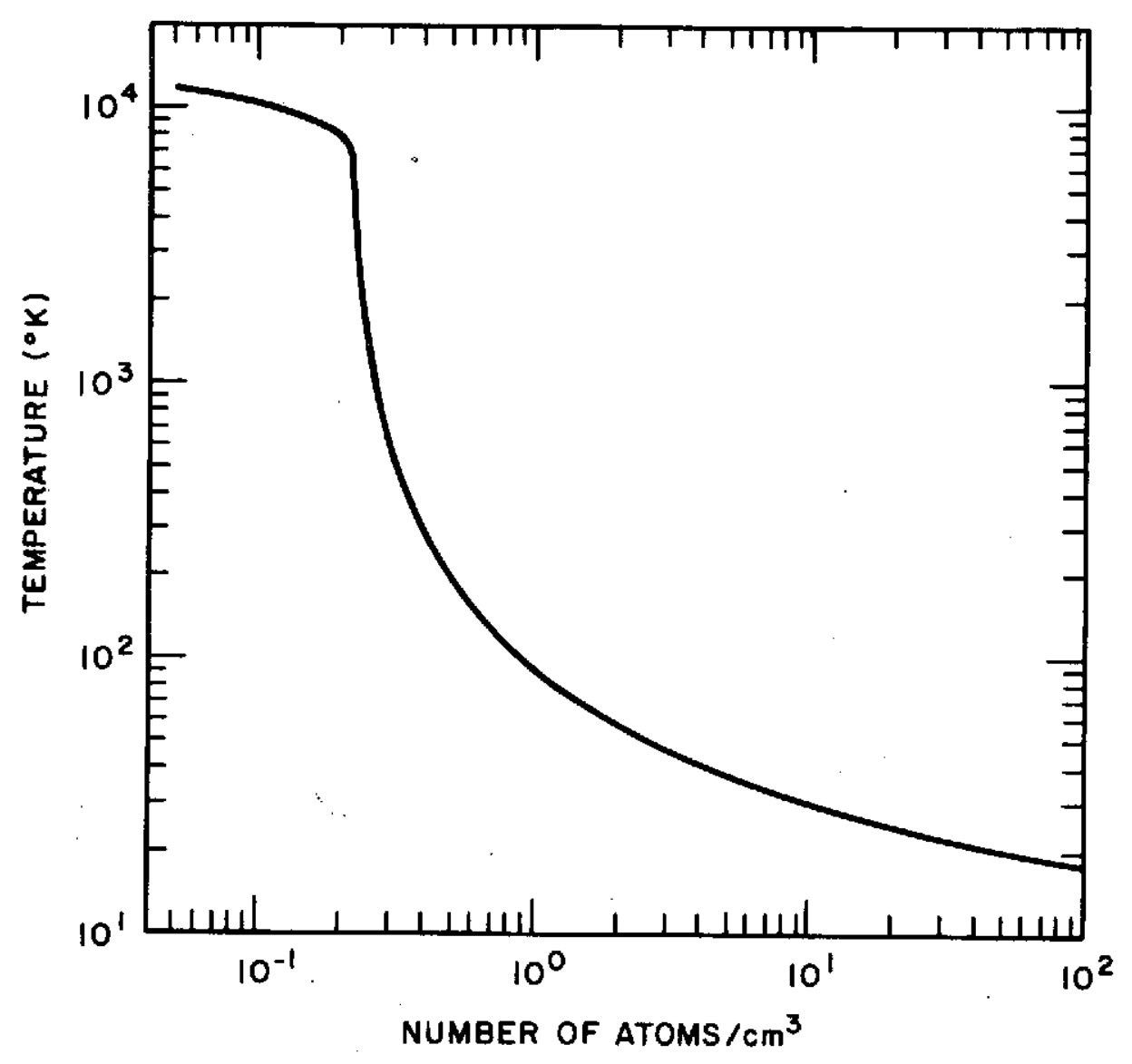

Fig. 6.3: The thermal state of the interstellar gas as a function of gas density. 
against the number density of interste1lar gas atoms. One can see from this figure that the thermal phases, which are not very sensitive to the change in the number density, around $10^{4 \circ} \mathrm{K}$ and below $10^{2 \circ} \mathrm{K}$ are stable. These phases occur at densities $n \sim 10^{-1}$ atoms $\mathrm{cm}^{-3}$ (which is typical of the intercloud medium) and $\mathrm{n} \sim 10$ atoms $\mathrm{cm}^{-3}$ (which is characteristic of the clouds respectively; the intermediate state is obviously unstable. It has also been shown by Field et al (1969) that the equilibrium temperature critically depends upon the abundance of cooling agents 1 ike $\mathrm{C}^{+}, 0^{\circ}$, $\mathrm{Si}^{+}$and $\mathrm{Fe}^{+}$in the gas, and that their depletion in dense clouds as a result of accretion of these trace elements in the grains can Increase the temperature of the cold phase considerably.

The above calculations on the thermal equilibrium of interstellar gas suggest that the ionization rate required is $\sim 10^{-15} \mathrm{sec}^{-1}$. Since the rate of loss of energy through ionization increases as the energy of the particle decreases, only very low energy cosmic rays below $50 \mathrm{MeV} / \mathrm{n}$ are of importance; here heavy nuclei contribute substantfally. It has also been suggested (Silk and Werner, 1970) that low energy $x$-rays in the region 100-250 eV, which are effectively absorbed in the interstellar medium, could also be an important source of heating and Ionization. However, the recent observations by Copernicus satellite (Rogerson et a1, 1973) indicate that low energy cosmic rays and $x$-rays cannot be the main source of heating of interstellar gas, because they lead to higher Ionization states which are not observed, and set a limit of $\sim 10^{-18} \mathrm{sec}^{-1}$ for the rate of ionization by these processes. It may perhaps be relevent to consider quantitatively the mechanism suggested by Wentzel (1971), In which heating of gas results from the dissipation of hydromagnetic waves created by cosmic rays during their propagation in 
Interstellar space (Section 3.2.2); however, since bulk of the cosmic rays have energies $\gtrsim 1 \mathrm{GeV}$, the heating by this process is associated with very little ionization.

\section{Acknowledgement}

One of us (SAS) wishes to acknowledge that this work was started while he was in Bombay and completed during his stay in Maryland, where it was supported in part by NASA Grants NAR 21-002-066, NAR 21-002-224 and NAR 21002-316, and NSF Grant GP25305. 


\section{References}

Abraham, P.B., K.A. Brunstein, and T.L. Cline, 1966, Phys. Rev. 150, 1088.

A1zu, H., Y. Fujimoto, S. Hasegawa, M. Koshiba, I. Mito, J. Nishimura, K. Yoko1, 1960, Suppl. Prog. Theor. Phys., 16, 54.

A1zu, H., Y. Fujimoto, S. Hasegawa, M. Koshiba, I. Mito, J. Nishimura, K. Yokot and M. Schein, 1961, Phys. Rev. 121, 1206.

Alfven, H., 1949, Phys. Rev. $\underline{75}, 1732$.

Alfven, H., 1950, Phys. Rev., 77, 375.

Alfven, H., 1965, Rev. Mod. Phys. 37, 652.

Alexander, J.K., L.W. Brown, T.A. Clark, R.G. Stone and R.R. Weker, 1969, Astrophys. J. (letters), 157, L163.

Alexander, J.K., L.W. Brown, T.A. Clark and R.G. Stone, 1970, Astron. Astrophys., $\underline{6}, 476$.

Alutia, H.R., 1972, Astrophys. Letters, 12, 237.

Allen, C.W., 1965, "Astrophysical Quantities", The Athlone Press.

Anand, K.C. and S.A. Stephens, 1971, Proc. 12th Int. Conf. on Cosmic Rays, Hobart; $7,2542$.

Anand, K.C. and S.A. Stephens, 1972, Astrophys. Sp. Sci: 18, 387.

Anand, K.C., R.R. Daniel and S.A. Stephens, 1968a, Proc. Ind. Acad. Sci., $67 A, 267$.

Anand, K.C., R.R. Danie1 and S.A. Stephens, 1968b, Nature, 217, 25.

Anand, K.C., R.R. Daniel and S.A. Stephens, 1968c, Proc. Ind. Acad. Sci., $68 \mathrm{~A}, 219$.

Anand, K.C., S. Blswas, P.J. Lavakare, S. Ramadura1, N. Sreenivasan, V.S. Bhatia, V.S. Chohan and S.D. Pabb1, 1969, Proc. Ind. Acad. Sci.; 70A, 201.

Anand, R.C., R.R. Danie1 and S.A. Stephens, 1973a, Proc. 13th Int. Conf. on Cosmic Rays, Denver, 1 , 355.

Anand, K.C̣., R.R. Daniel and S.A. Stephens, 1973b, Pramana, 1, 1.

Anders, E., 1965, Sp. Sc1. Rev., 3, 583. 
Andrew, B.H., 1966, Mon. Not. Roy. Astron. Soc., 132, 79.

Apparao, M.V.K. and S. Ramadura1, 1967, J. Geophys. Res. 72, 5991.

Apparao, M.V.K., 1967, Nature, 215, 727.

Apparao, M.V.K., 1973, Proc. 13th Int. Conf. on Cosmic Rays, Denver, 1 , OG375 (revised).

Arnett, W.D., 1969, Astrophys. J., 157, 1369.

Arnett, W.D, and D.D. C1ayton, 1970, Nature, 227, 780 .

Arp, H.C., 1965, in "Galactic Structure" ed. A. Blaauw and M. Schmidt, (University of Chicago Press), p. 401.

Atallah, K., T.F. Cleghorn, A. Modlinger and W.K.H. Schmidt, 1973, Proc. 13th Int. Conf. on Cosmic Rays, Denver, $1,208$.

Audouze, J., M. Epherre and H. Reeves, 1967, Nucl. Phys. A94, 144.

Audouze, J. and C.J. Cesarsky, 1973, Nature Physical Sc1. 241, 98.

Badhwar, G.D., R.R. Daniel and B. Vijayalakshmi, 1962, Prog. Theor. Phys. $28,607$.

Badhwar, G.D. and R.R. Danie1, 1963, Prog. Theor. Phys., 30, 615.

Badhwar, A.D. and R.W. Osborn, 1973, Proc. 13th Int. Conf. on Cosmic Rays, Denver, 1, 195; 1974, Astrophys. Sp. Sci. 28, 101.

Badhwar, G.D., C.L. Deney, R.R. Dennis and M.F. Kap1on, 1967, Phys. Rev., $\underline{163}, 1327$.

Badhwar, A.D., R.L. Golden and J.L. Lacy, 1973, Bul1, Am. Astron. Soc. $\underline{5}, 473$.

Baity, W.H., B.J. Teegarden, J.A. Lezniak and W.R. Webber, 1971, Astrophys . $\mathrm{J} ., 164,521$.

Balasubrahmanyan, V.K. and J.F. Ormes, 1973, Astrophys. J. 186, 109 .

Balasubrahmanyan, V.K., E. Boldt and R.A.R. Palmelra, 1965, Phys. Rev'., 140, B1157.

Balasubrahmanyan, V.K., H.E. Hagge, A.H. Ludwig and R.B. McDonald, 1966, J, Geophys. Res. 71, 1771 .

Balasubrahmanyan, V.K., J.F. Ormes and R. Ramaty, 1973, Proc. 13th Int. Conf. on Cosmic Rays, Denver, $1,163$.

Baldwin, J.E., 1855a, Mon. Not. Roy. Astron. Soc., 115, 684.

Baldwin, J.E., 1955b, Mon. Not. Roy. Astron. Soc., 115, 690. 
Baldwin, J.E., 1967, I.A.U. symposium No. 31, Ed. H. van Woerden (Academic Press), p. 337.

Beck, F. and F. Y1ou, 1968, Astrophys. Letters; $1,75$.

Beedle, R.E., J.A。 Lezniak, J.A. Rockstroch and W.R. Webber, 1970, Acta. Phys. Acad. Sc1. Hungaricae, 29, Supp1. 1, 131.

Bennett, K.,.:P. Penengo, A.K. Rochester, T.R. Sanderson and R.K. Sood, 1972, Nature, 238, 31 .

Berge, G.L. and Sielstad, G.A., 1967, Astrophys. J., 148, 367.

Berkey, G.B. and Shen, C.S., 1969, Phys. Rev., 188, 1994.

Bertsch, D.L., C.E. Fichte1 and D.V. Reames, 1972, Astrophys. J. 171, 169.

Beuermann, K.P., C.J. Rice, E.C. Stone and R.E. Vogt, 1970, Acta. Phys. Acad. Sc1. Hungaricae, 29, Supp1. 1, 173.

Bhabha, H.J., 1938, Proc. Roy. Soc. (London) Al64, 257.

Bhatia, V.S., S. Biswas and S. Ramadurai, 1970, Sp. Research, 10, 956.

Biermann, L. and L. Davies, 1960, Z. Astrophys. 51, 19.

Bignami, A.F. and C.E. F1chte1, 1974, Astrophys. J. (Letters), 189, L65.

Binns, W.R., J.I. Fernandez, M.H. Israel, J. Klarmann, R.C. Mach1 and R.A. Mewaldt, 1973, Proc. 13th Int. Conf. on Cosmic Rays, Denver; 1, 260.

Biswas, S. and E.C. Fichtel, 1965, Sp. Sci. Rev. 4, 709.

Biswas, S. and S. Ramadurai, 1971, Proc. 12th Int. Conf. on Cosmic Rays, Hobart, $1,162$.

Biswas, S, and S. Ramadurai, 1973, Proc. 13th Int. Conf. on Cosmic Rays, Denver, 1,260 .

Biswas, S., S. Ramadurai and N. Sreenivasan, 1966, Phys. Rev., 149, 1027.

Biswas, S., S. Ramadurai and N. Sreenivasan, 1967, Phys. Rev., 159, 1063.

Black, J.M. and G.G. Fazio, 1973, Astrophys. J. (Letters), 185, L7.

Blanford, G.E., M.W. Friedlander, J. Klarmann, S.S. Pomeroy, R.M. Walker, J.P. Wefel, P.H. Fowler, J.M. Kidd, E.J. Kobetich, R.T. Moses and R.T. Throne, 1973 a, Phys. Rev. D8, 1707.

Blanford, G.E., M.W. Friedlander, J. Klarmann, R.M. Walker and J.P. Wefe1, 1973b, Phys. Rev. D8, 1722. 
Bodansky, D., D.D. Clayton and W.A. Fowler, 1968, Astrophys. J. Supp1. 16, 299.

Bogomolov, E.A., N.D. Lubyanaya and V.A. Romanov, 1971, Proc. 12th Int. Conf. on Cosmic Rays, Hobart, 5, 1730.

Bracces1, A. and G. Vespigani, 1964, Nuovo Cimento, 31, 310.

Bradt, H.L. and B. Peters, 1950, Phys. Rev. $\underline{80}, 943$.

Brecher, K. and G.R. Burbridge, 1972, Astrophys. J. 174, 253.

Bridle, A.H., 1967, Mon. Not. Roy. Astron. Soc., 132, 79.

Bridle, A.H., 1968, Nature; 219, 1136.

Brown, J.W., E.C. Stone and R.E. Vogt, 1973a, Proc. 13th Int. Conf. on Cosmic Rays, Denver, 1,484 .

Brown, J.W., E.C. Stone and R.E. Vogt, 1973b, Proc. 13th Int. Conf. on Cosmic Rays, Denver, 1,556 .

Bulanov, S.V., V.A. Doge11 and S.I. Syrovatski1, 1972, Kosmich Issled, 10, 532 and 721.

Burb1dge, G.R., 1962, Prog. Theor. Phys. 27, 999.

Burbidge, G.R. and F. Hoyle, 1957, Nuovo Cimento, 4, 78.

Burbidge, G.R. and F. Hoyle, 1964, Proc. Phys. Soc., 84, 141.

Burger, J.J., 1971, Astrophys. J., 166, 651.

Burger, J.J. and B.N. Swanenburg, 1973, Proc. 13th Int. Conf. on Cosmic Rays, Denver; 5, 3061 .

Burger, J.J., S.A. Stephens and B.N. Swanenburg, 1970, Astrophys. Sp. Sci. 8, 20 .

Casse, M. and P. Goret, 1973, Proc. 13th Int. Conf. on Cosmic Rays, Denver, $\underline{1}, 584$.

Casse, M., L. Koch, N. Lund, J.P. Meyer, B. Peters, A. Soutoul and S.N. Tandon, 1971, Proc. 12th Int. Conf. on Cosmic Rays, Hobart, 1, 241.

Caval1o, G. and R.J. Gould, 1971, Nuovo. Cimento, 132, 77.

Clark, G.W., G.P. Garmaire and W.L. Kraushaar, 1968, Astrophys. J. (Letters), 153, L203.

Clayton, D.D., 1968, "Principles of Stellar Evolution and Nucleosynthesis" (McGraw-H111). 
Cesarsky, C.J. and J. Audouze, 1973, Proc. 13th Int. Conf. on Cosmic Rays, Denver, 1,189 .

Chen, M.C., 1972, II Nuovo Cimento, 8B, 343 .

Chocate, L.M. and J.R. Wayland, 1972, Astrophys. Sp. Sc1. 19, 195.

Cline, T.L. and Hones, E.W., 1970, Acta Phys. Acad. Sci. Hungaricae, 29, Supp1: 1, 159 .

Cline, T.I. and G. Porreca, 1970, Acta. Phys. Acad. Sct. Hungaricae, 29, Supp1. 1, 145.

Colgate, S.A., 1968, Can. J. Phys. 46, S496.

Colgate, S.A. 1970, Astrophys. Sp. Sci. $\underline{8}, 457$.

Coms tock, G.M., 1969, Astrophys. J., 155, 619.

Comstock. G.M., K.C. Hsleh and J.A. Simpson, 1972, Astrophys. J. 173, 691.

Comstock, G.M., C.Y. Fan and J.A. Simpson, 1969, Astrophys. J., 155, 609 .

Constain, C.H., 1960, Mon. Not. Roy. Astron. Soc., 120, 248.

Cowsik, R., 1971, Proc. 12th Int. Conf. on Cosmic Rays, Hobart, $1,329$.

Cowsik, R., 1972, Nature Physical Sci. 239, 41.

Cowsik, R. and Yash Pal, 1969, Phys. Rev. Letters, 22, 550.

Cowstk, R. and L.W. Wilson, 1973, Proc. 13th Int. Conf. on Cosmic Rays, Denver, 1,500 .

Cowsik, R. and J. Mitteldorf, 1974, Astrophys. J. 189, 51.

Cowsik, R. Yash Pal. S.N. Tandon and R.P. Verma, 1967, Phys. Rev. 158, 1238.

Cowsik, R. Y. Pal, S.N. Tandon and R.P. Verma, 1970, Acta Phys. Acad. Sci. Hungaricae, 29, Supp1. 1, 439 .

Crawford, H.J., P.B. Price and J.D. Sull1van, 1972, Astrophys. J. (Letters), 175, L149.

Cummings, A.C., E.C. Stone and R.E. Vogt, 1973a, Proc. 13th Int. Conf. on Cosmic Rays, Denver, 1,340 .

Cummings, A.C., E.C. Stone and R.E. Vogt, 1973b, Proc. 13th Int. Conf. on Cosmic Rays, Denver, $\underline{1}, 335$.

Dah1backa, G.H., P.S. Freier and C.J. Waddington, 1973, Astrophys. J. 180, 371. 
Dalgarno, A. and R.A. McCray, 1972, Ann. Rev. Astron. Astrophys. 10, 375. Damle, S.V., 1968, Ph.D. Thes1s, University of Bombay (unpublished). Daniel, R.R. and N, Durgaprasad; 1966, Prog. Theor. Phys 35, 36. Daniel, R.R. and S.A. Stephens, 1967, Proc. Ind. Acad. Sc1. 65A, 319. Danie1, R.R. and S.A. Stephens, 1970, Sp. Sci. Rev., 10, 599.

Danie1, R.R. and S.A. Stephens, 1970a, Acta Phys. Acad. Sc1. Hungaricae, 29, Suppl. 1, 53 .

Danie1, R.R. and S.A. Stephens, 1974, Rev. Geophys. Sp. Phys. 12, 233. Denisse, J.F., E. Leroux and J.L. Steinberg, 1955, Compt. Rend., 240, 278. Dłcke, R.H., P.J.E. Peebles, P.G. Ro11 and D.J. Wilkinson, 1965, Astrophys. J., 142,414 .

Dooher, J., 1973, Phys. Rev. D, 1406.

Durgaprasad, N., 1967, J. Geophys. Res., 72, 965.

Ear1, J.A., 1974, Astrophys. J. 188, 379.

Ear1, J.A. and A.M. Lencheck, 1969, Astrophys. J. 157, 87.

Ear1, J.A., D.E. Neely and T.A. Rygg, 1972, J. Geophys. Res. 77, 1087.

E11iot, H., T. Tambyahpillai, P.S. Peacock, 1970, Acta Phys. Acad. Sci. Hungar1cae, 29, Supp1. 1, 491.

Elvius, J., 1965, in "Galactic Structure" ed. A. Blaauw and M. Schmidt, (University of Chicago Press), p. 41.

Evanson, P. 1972, Astrophys. J. 176, 797.

Fan, C.Y., G. Gloeckler and J.A. Simpson, 1966, Phys. Rev. Letters, $16,813$.

Fan, C.Y., G. Gloeckler and J.A. Simpson, 1968, Canad. J. Phys., 46, S548.

Fanselow, J.L., R.C. Hartman, R.A. Hildebrand and P. Meyer, 1969, Astrophys. $\mathrm{J} ., 158,771$.

Felten, J.E., 1966, Astrophys. J., 145, 589.

Felten, J.E. and P. Morrison, 1966, Astrophys. J., 146, 686.

Fermi, E., 1934, Z. Phys. 88, 161.

Ferint, E., 1949, Phys. Rev. 75, 1169. 
F1chte1, E.C. and D. Reames, 1966, Phys. Rev., 149, 995.

Fichte1, E.C. and D. Reames, 1968, Phys. Rev., 175, 1564.

F1chte1, C.E., R.C. Hartman and D.A. Kniffen, 1973, Proc. 13th Int. Conf. on Cosmic Rays, Denver, 1, 302.

Fleld, G.B., 1970, in "Interstellar Gas Dynamics" ed. H.J. Habing (Reidel Pub. Co.), p. 51.

Fleld, G.B., D.W. Goldsmith and H.J. Habing, 1969, Astrophys. J. (Letters), 155, L149.

Finkel, R.C., J.R. Arnold, M. Imamura, R.C. Reddy, J.S. Fruchter, H.H. Loosl1, J.C. Evans, A.C. Delany and J.P. Shedlousky, 1971, Proc. 2nd Lunar Sc1. Conf. 2, 1773 .

Fontes, P., C. Perron, J. Lestringuez, F. Ylou and R. Bernas, 1971, Nucl. Phys. A165, 405.

Fowler, P.H., 1973, Proc. 13th Int. Conf. on Cosmic Rays, Denver, $5,3627$.

Fowler, P.H., V.M. Clapham, V.G. Cowen, J.M. Kidd and R.T. Moses, 1970, Proc. Roy. Soc. (London), A318, 1 .

Fowler, P.H., R.T. Thorne, A. Muzumdar, C. O'Caellaigh, D. O'Sullivan, X.V. Rao and A. Thompson, 1973, 13th Int. Conf. on Cosmic Rays, Denver, 5, 3239 .

Fradkin, M.I., 1956, Soviet Phys. JETP, 2, 87.

Freier, P.S., J.S. Rao and C.J. Waddington, 1966, Proc. 10th Int. Conf. on Cosmic Rays (London), $1,38$.

Freler, P.S. and C.J. Waddington, 1968, J. Geophys, Res., 73, 4261.

Frye, G.M., P.A. Albats, A.D. Zych, J.A. Statb, V.D. Hopper, W.R. Rawlinson and J.A. Thomas, 1971, Nature, 231, 372.

Gaisser, T.K. and R.H. Maurer, 1973, Phys, Rev. Letters, 30, 1264.

Gamow, G., 1948, Phys. Rev. 74, 505.

Ganguli, S.N., N. Kameshwar Rao and M.S. Swamy, 1967, Proc. Ind. Acad. Sc1., $65 \mathrm{~A}, 19$.

Garcia-Munoz, M., 1973, Proc. 13th Int. Corif. on Cosmic Rays, Denver, 5, 3513.

Garcia-Munoz, M. and J.A. Simpson, 1970, Acta Phys. Acad. Sc1. Hungaricae, 29, Supp1. 1, 317.

Garcia-Munoz, M., A.M. Mason and J.A. Simpson, 1973, Proc, 13th Int. Conf. on Cosmic Rays, Denver, OG-128. 
Gelss, J., 1963, Proc. 9th Int. Conf. on Cosmic Rays, Jalpur, 3 , 434.

Getmantsev, G.G., 1963, Soviet Astron. AJ, 6, 477.

Getmantsev, G.G., V.S. Karavanov, Yu-S. Korobkov and A.F. Tarasov, 1968, Astron. Zh. SSSR, 45, 936.

Ginzburg, V.L., 1967, IAU symposium No. 31, ed. H. van Woerden (Academic Press), p. 365.

Ginzburg, V.L., and S.I. Syrovatskil, 1964, "Origin of Cosmic Rays", Pergamon Press.

Ginzburg, V.L. and S.I. Syrovatski1, 1965, Ann. Rev. Astron. Astrophys. 3 , 297.

Ginzburg, V.L. and S.I. Syrovatskit, 1966, Proc. 10th Int. Conf. on Cosmic Rays, London, 1 , 53.

Ginzburg, V.L. and Syrovatski1, 1968, Proc. 11th Int. Conf. on Cosmic Rays, Calgary, Invited and Rapporteur talks, p. 53.

Ginzburg, V.L. and Ya. M. Khazan, 1972, Astrophys. Letters, 12, 155.

Gleeson, L.J. and I.H. Uhrch, 1971, Astrophys. Sp. Sc1., 11, 288.

Gloeckler, G. and J.R. Joklpii, 1970, Acta Phys. Acad. Sc1. Hungaricae, 29, Supp1. 1, 541 .

Golden, R.L.J.H. Adams, A.D. Badhwar, C.L. Deney, H.H. Heckman and P.L. Lindstrom, 1973a, Reported at 13th Int. Conf. on Cosmlc Rays, Denver, OG-543.

Golden, R.L., J.H. Adams, A.D. Badhwar, C.L. Deney, H.H. Heckman and P.L. Lindstrom, 1973b, Reported at 13th Int. Conf. on Cosmlc Rays, Denver, OG-544.

Goldsmith, D.W., H.J. Habing and G.B. Field, 1969, Astrophys. J., 158, 173.

Goldstein, M.L., R. Ramaty and L.A. Fisk, 1970, Phys. Rev. Letters, 24, 1193.

Gould, R.J. and G.R. Burbidge, 1965, Ann. Astrophys., 28, 171.

Greenberg, J.M., 1967, In Transactions I.A.U., 13A, Ed. L. Perck, (D. Re1de1 Publishing Company), p. 752.

Greenberg, J.M., 1968, in "Nebula and Interstellar Matter", Ed. by B.A. Middlehurst and L.H. Aller, (University of Chicago Press), p. 1.

Greenberg, D.W., 1969, Astrophys. J., 155, 451.

Greenhil1, J.A., A.R. Clarke and H. E11lot, 1971, Nature 230, 171. 
Grigorov, N.L., D.A. Zhuraulev, M.A. Kondrat'eva, I.D. Rapaport and I.A. Savenko, 1961, Artificial Earth Satellites, 10, 96.

Habing, H.J., 1968, Bull. Astron. Inst., Neth., 19, 421.

Hagge, D.E., V.K. Balasubrahmanyan and F.B. McDona1d, 1968, Canad, J. Phys. 46, 5539 .

Ha11, J.S., 1949, Sclence, 109, 166.

Hanbury Brown, R., R.D. Davies and C. Hazard, 1960, Observatory, $\underline{80}, 191$.

Hartmann, J. 1904, Astrophys. J., 19, 268.

Havnes, 0., 1971, Nature, 229, 548.

Havnes, 0., 1973, Astron, Astrophys. 24, 435.

Hayakawa, S., K. Ito and Y. Terashima, 1958, Supp1. Prog. Theor. Phys., 6, 1.

Hayakawa, S., and M. Matsuoka, 1964, Suppl. Prog. Theor. Phys. 30, 204.

Heiles, C., 1967, Astrophys. J. 148, 299.

H111, E.R., O.B. Slee and B.Y. Mills, 1958.

H1ltnex, W.A., 1949, Sclence, 109, 165.

Hjellming, R.M., C.P. Gordon and K.J. Gordon, 1969, Astron. Astrophys., $\underline{2}$, 202.

Hoffmann, D.J., and J.R. Winckler, 1967, P1an. Sp. Sc1., 15, 715.

Holmes, J.A., 1974, Mon. Not. Roy. Astron. Soc. 166, 155.

Hovestadt, D., P. Meyer and P.J. Schmidt, 1971, Astrophys. Letters, $\underline{9}, 165$.

Hovestadt, D., 0. Vollmer, G.Gloeckler and C.Y. Fan, 1973, Phys. Rev. Letters, 31, 650 .

Howe11, T.F., 1970, Astrophys. Letters, $\underline{6}, 45$.

Hoyle, F. and J.G. Ireland, 1961, Mon. Not. Roy. Astr. Soc., 122, 35.

Hsieh, K.C. and J.A. Simpson, 1969, Astrophys. J. (Letters), 158, L37.

Hsieh, K.C. and J.A. Simpson, 1970, Astrophys. J. (Letters), 162, L197.

Hsieh, K.C.,.A.M. Mason and J.A. Simpson, 1971, Astrophys. J. 166, 221.

Hurford, A.J., R.A. Mewaldt, E.C. Stone and R.E. Vogt, 1973, Proc. 13th Int. Conf. on Cosmic Rays, Denver, $1,93$. 
Hurford, G.J., R.A. Mewaldt, E.C. Stone and R.E. Vogt, 1973a, Proc. 13th Int. Conf. on Cosmic Rays, Denver, $1,324$.

Hurford, G.J., R.A. Mewaldt, E.C. Stone and R.E. Vogt, 1973b, Proc. 13th Int. Conf. on Cosmic Rays, Denver, 1,330 .

Ipavich, F.M. and M. Lenchek, 1970, Phys. Rev. Letters, 2D, 266.

Ishii, C., T. Kobayashi, N. Shigihara, K. Yoko1, M. Matsuo, J. Nish1mura, T. Taira and K. Niu, 1973, Proc. 13th Int. Conf. on Cosmic Rays, Denver, 5, 3073 .

Ivanova, N.S., Yu F. Gagarin and V.N. Kulikov, 1968, Cosmic Research, $\underline{6}, 69$. Jeans, J.M. , 1925, Nature, 116, 861.

Jeans, J.H., 1928, "Astronomy and Cosmology", Cambridge University Press, p. 337.

Jenkins, E.B., J.F. Drake, D.C. Morton, J.B. Rogerson, L. Spitzer and D.G. York, 1973, Astrophys. J. (Letters), 181, L 122.

Johnson, T.H., 1938, :Rev, Mod. -,Phys. I0, 193.:

Jokipi1, J.R. and P. Meyer, 1968, Phys. Rev. Letters, 20, 572.

Jokipil, J.R. and E.N. Parker, 1968, Phys, Rev. Letters, 21, 44.

Jokipi1, J.R. and E.N. Parker, 1969a, Astrophys. J., 155, 777.

Jokipil, J.R. and E.N. Parker, 1969b, Astrophys. J., 155, 799.

Jokipif, J.R. and I. Lerche, 1969, Astrophys. J. 157, 1137.

Jones, F.C., 1970a, Acta Phys. Acad. Sci. Hungaricae, 29, Supp1. 1, 23.

Jones, F.C., 1970b, Phys, Rev. D2, 2787.

Jones, F.C., 1971, Proc. 12th Int. Conf. on Cosmic Rays, Hobart, $1,396$.

Juliusson, E., 1974, Astrophys. J. 191, 331 .

Jullusson, E. and P. Meyer, 1973, Astrophys. Letters, 14, 153.

Juliusson, E., P. Meyer and D. Mu1ler, 1972, Phys. Rev. Letters, 29, 445.

Kaiser, T.B. J.R. Wayland and G. Gloeckler, 1972, Phys. Rev. D5, 307.

Ke11man, S.A. 1972a, Astrophys.J, 175, 353.

Kellman, S.A. 1972b, Astrophys. J. 175, 363. 
Kaplon, M.F. and G. Skadron, 1966, Rev. Geophys. 4 , 177.

Kepner, M., 1970, Astron. Astrophys: 5, 444.

Kerr, F.J. and G. Westerhout, 1965, in "Galactic Structure", ed. A. Blaauw and M. Schmidt, (University of Chicago Press), p. 167.

Kerr, F.J., 1967, I.A.U. Sympostum No. 31, Ed. H. van Woerden (Academic Press), p. 239.

Kniffen, D.A., R.C. Hartman, D.J. Thompson and C.E. Fichte1, 1973, Astrophys. J. (Letters); 186, L105.

Komesaroff, M.M., 1966, Aust. J. Phys., 19, 75.

Korchak, A.A. and S.I. Syrovatsk11, 1958, Soviet Phys. Dok1: 3, 983.

Kozlovsky, B. and R. Ramaty, 1973, Nature Phys. Sc1. 246, 55.

Kraus, J.D. and H.C. Ko, 1955, Nature, 175, 159.

Kraushaar, W.L., A.W. Clark, G.P. Garmire, R. Barken, P. Higbie, V. Leong and T. Thorsos, 1972, Astrophys. J. 177, 341.

Krishnaswamy, K.S., 1970, Astrophys. Sp. Sc1.; 6, 474.

Kristiansson, K., 1966, Arkiv Fysik, 32, 285.

Kristiansson, K., 1971, Astrophys. Sp. Sc1. 14, 485.

Kristiansson, K., 1972, Astrophys. Sp. Sc1. 16, 405.

Kulsrud, R. and W.P. Pearce, 1969, Astrophys. J., 156, 445.

La1, D., 1966, Proc. 10th Int. Conf. on Cosmic Rays, London, $\underline{1}, 81$.

Lamaitre, G. 1931, Nature, 128, 704.

Lampton, M., D.W. Green and C.S. Bowyer, 1971, Nature, 230, 448.

Large, M.I., D.S. Mathewson and C.G.J. Haslam, 1961, Mon. Not. Roy. Astron. Soc., 123,113 .

Laster, H., 1964, Phys. Rev. 135, B1274.

Ledoux, P.,1951, Ann. d'Astrophys. 14, 438.

Lequeux, J., 1967, I.A.U. Symposium No. 31, Ed. H. van Woerden (AcademIc Press), p. 393.

Lequeux, J., 1969, "Structure and Evolution of Galaxies", (Gordon and Breach Science Publishers). 
Levy, D.J. and D.W. Goldsmith, 1972, Astrophys. J. 177, 341.

Lin, R.P., K.A. Anderson and T.L. Cline, 1972, Phys. Rev. Letters, 29, 1035.

Lingenfelter, R.E., R. Ramaty and L.A. Fisk, 1971, Astrophys. Letters, 8 , 93.

Lipschutz, M.E., P. Singer and E. Anders, 1965, J. Geophys. Res., 70, 1473.

Longair, M.S., 1970, Mon. Not. Roy. Astron. Soc. 150, 155.

Malmquist, L., 1967, Arkiv. Fur. Phys., 34, 32.

Marasch1, L., G.C. Perola and S. Schwarz, 1968, Nuovo Cimento, 53B, 443.

Maraschi, L. and A. Treves, 1970, Astrophys. Letters, $\underline{5}, 177$.

Marsden, P.L., R. Jakeways and I.R. Calder, 1971, Proc. 12th Int. Conf. on Cosmic Rays, Hobart, 1, 110.

Mason, G.M., 1972, Astrophys. J. 171, 139.

Mathewson, D.S., J.R. Healey and J.M. Rome, 1962, Aust. J. Phys., 15, 354 .

Mathewson, D.S. and D.C. N1chols, 1968, Astrophys. J. (Letters), 154, L11.

Mathewson, D.C., 1968, Astrophys. J. (Letters), 153, L47.

McDonald, F.B., B.J. Teegarden, J.H. Trainor and W.R. Webber, 1974, Astrophys. J. (Letters), 187, L105.

Meegan, C.A. and J.A. Ear1, 1973, Proc. 13th Int. Conf. on Cosmic Rays, Denver; $5,3067$.

Meneguzzi, M., 1973a, Nature Phys. Scl. 241, 100.

Meneguzz1, M., 1973b, Proc. 13th. Int. Conf. on Cosmic Rays, Denver, $1,378$.

Metzger, A.E., E.C. Anderson, M.A. van Dilla and J.R. Arnold, 1964, Nature, 204, 766 .

Meyer, P., 1969, Ann. Rev. Astron. Astrophys. 2, 1.

Meyer, P., 1971, Proc. 12th Int. Conf. on Cosmlc Rays, Hobart, Invited and Rapporteur talks, p. 241.

Meyer, J.P., 1971, Proc. 12th Int. Conf. on Cosm1c Rays, Hobart; 7, 2565.

Meyer, J.P., D.E. Hagge, and F.B. McDonald, 1968, Canad, J. Phys. 46, S503.

Mi11s, B.Y., 1967, I.A.U. Sumpostum No. 31, Ed., H. van Woerden, (Academlc Press), p. 363. 
Milne, D.K, and H.R. Hil1, 1969, Aust. J. Phys. 22, 211.

Mogro-Campero, A. and J.A. Simpson, 1972a, Astrophys. J. (Letters), 171, L5.

Mogro-Campero, A. and J.A. Simpson, 1972b, Astrophys. J. (Letters), 177, L37.

Mogro-Campero, A., N. Schofield and J.A. Simpson, 1973, Proc. 13th Int.

Conf. on Cosmic Rays, Denver, $1,140$.

Moron, M., 1965, Mon. Not. Roy. Astron. Soc., 129, 447.

Morton, D.C., J.F. Drake, E.B. Jenkins, J.B. Rogerson, L. Spitzer and D.G. York, 1973, Astrophys. J. (Letters), 181, L103.

Mueh1ner, D. and R. Welss, 1970, Phys. Rev. Letters, 24, 742.

Mulier, D. and P. Meyer, 1973, Astrophys. J. 186, 841.

0'Connel1, R.F. and S.D. Verma, 1969, Phys. Rev. Letters, 22, 1443.

O'De11, F.W., M.M. Shapiro, R. S1lberberg and C.A. Tsao, 1971, Proc. 12th

Int. Conf. on Cosmic Rays, Hobart, $1,197$.

O'De11, F.W., M.M. Shapiro, R. Silberberg and C.H. Tsao, 1973, Proc. 13th Int. Conf. on Cosmic Rays, Denver, $\underline{1}, 490$.

Okuda, H. and Y. Tanaka, 1968, Can. J. Phys., 46, S642.

Oort, J.H., 1938, Bull. Astr. Inst. Neth., 8, 233.

Oort, J.H., 1960, Bull: Astron. Inst. Neth., 15, 45.

Oort, J.H., 1969, Nature, 224, 1158.

Oort, J.H., 1973, I.A.U. Symposium on "Galactic Radio Astronomy", Maroochydore (under publication).

Ormes, J.F. and V.K. Balasubrahmanyan, 1973, Nature Phys. Sc1. 241, 95.

O'Sullivan, P.B. Price, E.K. Shirk, P.H. Fowler, J.M. Kidd, E.J. Kobetich and R. Thorne, 1971, Phys. Rev. Letters, 26, 463.

Pacheco, J.A.F., 1971, Astron. Astrophys. 13, 58.

Parker, E.N., 1963, "Interplanetary Dynamical Processes", Interscience Pub1ishers.

Parker, E.N., 1965, Astrophys. J., 142, 584.

Parker, E.N., 1968, in "Nebula and Interstellar Matter", Ed. B.A. Middlehurst and L.H. Aller, (University of Chicago Press), p. 707. 
Parker, E.N., 1968a, in "Colloquium on Cosmic Ray Studies", Ed. R.R. Danie1, P.J. Lavakare and S. Ramaduraf (Tata Institute of Fundamental Research), p. 202 .

Parker, E.N., 1969, Sp. Sc1. Rev. $\underline{9}, 651$.

Parthasarathy, R. and G.M. Lerfald, 1965, Mon. Not. Roy. Astron. Soc., 129, 395.

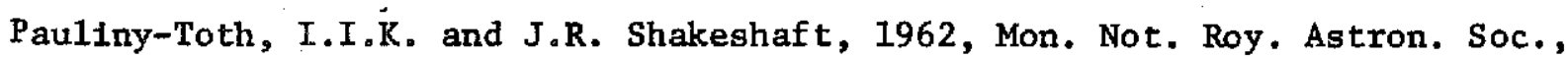
124,61 .

Peeble, P.J.E., 1971, "Physical Cosmology", Princeton Series, New Jersey.

Penzias, A.A. and R.W. Wilson, 1965, Astrophys. J., 142, 419.

Penzlas, A.A. and R.W. Wilson, 1966, Astrophys. J., 146, 666.

Perola, G.C., L. Scarsi and G. Sironi, 1967, Nuovo Cimento, 5213, 455.

Peters, B., 1963, Pontificia Acad. Sci. Seripta Varia, 25, 1.

Piddington, J.A. and G.H. Trent, 1956, Aust. J. Phys., $\underline{9}, 481$.

Pikelner, S.B., 1967, In Transactions of I.A.U., 13A, Ed. L. Perek, (D. Reide1 Publishing Company), p. 729.

Prentice, A.J.R. and D. Ter Haar, 1969, Nature, 222, 964.

Price, P.B., I. Hutcheon, R. Cowsik and D.J. Barber, 1971a, Phys. Rev. Letters, 26, 916 .

Price, P.B., P.H. Fowler, J.M. Kield, E.J. Kobetich, R.L. Flelschev, and G.E. Nichols, 1971b, Phys. Rev; D3, 815.

Price, P.B., J.H. Chan, D. O'Sullivan and A. Thompson, 1973, Proc. 13th Int. Conf. on Cosmic Rays, Denver, $1,146$.

Pskovski1, Y.P., 1965, Astr. Zhu., 42, 537, (Soviet Astr. A.J. 9, 919, 1966).

Purton, C.R., 1966, Mon. Not. Roy. Astron. Soc., 133, 463.

Puzanov, V.I., A.E. Salomonvich and K.S. Stankevich, 1968, Soviet Astron. A.J., $11,905$.

Raisbeck, G, and F. Yiou, 1973, Proc. 13th Int. Conf. on Cosmic Rays, Denver, $\underline{1}, 494$.

Ramadurai, S., 1970, Ph.D. Thesis, University of Bombay (unpublished).

Ramadurai, S. and S. Biswas, 1972, Astrophys. Sp. Sc1. 17, 467.

Ramaty, R. and R.E. Lingenfelter, 1966a, J. Geophys. Res., 71, 3687. 
Ramaty, R. and R.E. Lingenfelter, 1968, Phys. Rev. Letters, 20, 120.

Ramaty, R, and R.E. Lingenfelter, 1969, Astrophys. J., 155, 587.

Ramaty, R. and R.E. Lingenfelter, 1971, in "Isotopic Composition of the Primary Cosmic Radiation", ed. P.A. Dauber (Danish Space Research Institute), p. 203.

Ramaty, R., D.V. Reames, and R.E. Lingenfelter, 1970a, Phys. Rev. Letters, 24,913 .

Ramaty, R., F.W. Stecker and D. Misra, 1970b, J. Geophys. Res. 25, 1141.

Ramaty, R., T.L. Cline and L.A. Fisk, 1972, Phys. Rev. Letters; 29, 1039.

Ramaty, R., V.K. Balasubrahmanyan and J.F. Ormes, 1973, Science, 180, 731.

Ray, E.C., 1960, J. Geophys. Res. 65, 1125 .

Reames, D.V., 1971, Astrophys. J., 162, 837 .

Reames, D.V. and E.C. Fichtel, 1967, Phys. Rev. 162, 1291.

Reames, D.V. and C.E. Fichte1, 1968, Canadian J. Phys. 46, S544.

Rengarajan, T.N., S.A. Stephens and R.P. Verma, 1971, Proc. 12th Int. Conf. on Cosmic Rays, Hobart, $\underline{7}, 2606$.

Rengarajan, T.N., S.A. Stephens and R.P. Verma, 1973, Proc. 13th. Int. Conf. on Cosmic Rays, Denver, 1, 384.

Richtmyer, R.D. and E. Teller, 1949, Phys. Rev. 75, 1729.

Rogerson, J.B., D.G. York, J.F. Drake, E.B. Jenkins, D.C. Morton and L. Spitzer, 1973, Astrophys. J. (Letters), 181, L110.

Rosen, S., 1967, Phys. Rev. 158, 1227.

Rougoor, G.W., 1966, Astrophys. J., 144, 852.

Rougoor, G.W. and J.H. Oort, 1960, Proc. Nat. Acad. Sci., USA, 46, 1.

Rudstam, G., 1955, Phil. Mag. 46, 344.

Rudstam, G., 1966, Z. Naturforsch, 21A, 1027.

Scheaffer, O.A., R. Davis, R.W. Steenner, and D. Heymann, 1963, Proc.

Int. Conf. on Cosmic Rays, Jaipur, $\underline{3}, 480$.

Scheepmaker, A. and Y. Tanaka, 1971, Astron. Astrophys. 11, 53.

Schmidt, M., 1957, Bull. Astron. Inst. Neth., 13, 247.

Schmidt, M., 1965, in "Galactic Structure", Ed. A. Blaaw and M. Schmidt, (University of Chicago Press), p. 513. 
Sciama, D., 1962, Mon. Not. Roy. Astron. Soc., 123, 317.

Seeger, C.L., F.L.H.M. Stempers and N. Aarch, 1960, Phillips Tech. Rev., 21,317 .

Seeger, C.L., G. Westerhout, R.G. Convey and T. Hockema, 1965, Bull. Astron. Inst. Neth., 18, 11 .

Seeger, P.A., W.A. Fowler and D.D. Clayton, 1965, Astrophys. J. Supp1. 11, 121.

Setti, G. and L. Woltzer, 1971, Nature Phys. Sci., 231, 57.

Shain, C.A., 1958, Proc. I.A.U. Symposium on "Radio Astronomy", Paris, p. 328.

Shakeshaft, J.R., 1968, in "Colloquium on Cosmic Ray Studies", Ed. R.R. Dantel, P.J. Lavakare and S. Ramadurai, (Tata Institute of Fundamental Research), p. 131 .

Shapiro, M.M. and R. Silberberg, 1970, Ann. Rev. Nucl. Sci., 20, 324.

Shapiro, M.M., R. Silberberg and C.H. Tsao, 1970a, Acta Phys. Acad. Sci. Hungaricae, 29, Supp1. 1, 479 .

Shapiro, M.M., R. Silberberg and C.A. Tsao, 1970b, Acta Phys. Acad. Sci., Hungaricae, 29 , Supp1. $1,471$.

Shapiro, M.M., R. Silberberg and C.A: Tsao, 1973, Proc. 13th Int.: Conf . on Cosmic Rays, Denver, 1,578 .

Share, G.H., R.L. Kinzer and N. Seeman, 1974, Astrophys. J. 187, 45.

Shen, C.S., 1969, Phys. Rev. Letters, 22, 568.

Shen, C.S., 1970, Astrophys. J. (Letters), 162, L181.

Shen, C.S. and G.B. Berkey, 1968, Phys. Rev. 171, 1344 .

Shirk, E.K., P.B. Price, E.J. Kobetich, W.Z. Osborne, L.S. Pinsky, R.D. Eandi and R.B. Rushing, 1973, Phys. Rev. D7, 3220.

Shivanandan, K., J.R. Houch, and M.0. Harw1t, 1968, Phys. Rev. Letters, 20, 1460 .

Shklovsky, I.S., 1952, Astron. Ah. SSSR, 29, 418.

Silberberg, R. and C.H. Tsao, 1973a, Astrophys. J. Supp1. 25, 315.

Silberberg, R. and C.H. Tsao, 1973b, Astrophys. J. Supp1. 25, 335.

Silberberg, R., M.M. Shapiro and C.H. Tsao, 1973, Proc. 13th Int. Conf. on Cosmic Rays, 1 , 567 . 
Silk, J. and G. Strefgman, 1969, Phys. Rev. Letters, 23, 597.

Silk, J., and M. Werner, 1970, Nature Phys. Sci., 243, 134.

Silverberg, R.F., and R. Ramaty, 1973, Nature Phys. Sci., 243, 134.

Silverberg, R.F., J.F. Ormes and V.K. Balasubrahmanyan, 1973, Proc. Int. Conf. on Cosmic Rays, $1,347$.

Simnett, G.M. and F.B. McDonald, 1969, Astrophys. J., 157, 1435.

Simpson, J.A., 1971, Proc. 12th Int. Conf. on Cosmic Rays, Hobart, Invited and Rapporteur Papers, p. 324.

Sironi, G., 1965, Nuovo Cimento, $39,372$.

Sironi, G., 1974, Mon. Not. Roy. Astron. Soc. 166, 345.

Smith, F.G., 1968, Nature, 220, 891.

Smith, L.H., A. Buffington, G.F. Smoot, L.W. Alvarez and W.H. Wahlig, 1973a, Astrophys. J. 180,987 .

Smith, I.H., A. Buffington, C.D. Orth and G.F. Smoot, 1973b, reported at the Proc. Int. Conf, on Cosmic Rays, Denver, OG-128.

Smoot, G.F., A Buffington, C.D. Orth and L.H. Smith, 1973, Proc. Int. Conf. on Cosmic Rays, Denver, $\underline{1}, 225$.

Speller, R., T. Thambyahpillai and H. Elliot, 1972, Nature, 235, 25.

Spitzer, L., 1968a, in "Nebula and Interstellar Matter", Ed. by B.A. Middlehurst and L.H. Aller, (University of Chicago Press), p. 1.

Spitzer, L., 1968b, "Diffuse Matter in Space", Interscience Publishers.

Stecker, F.W., 1969, Nature, 222, 865.

Stecker, F.W., 1970, Astrophys. Sp. Sci., $\underline{6}, 377$.

Stecker, F.W., 1971, "Cosmlc Gamma Rays", NASA Scientific and Technical Publications, Washington, D.C.

Stecker, F.W., D.L. Morgan and J.H. Bredekamp, 1971, Phys. Rev. Letters, 27, 1469 .

Stecker, F.W., J.L. Puget, A.W. Strong and J.H. Bredekamp, 1974, Astrophys. J. (Letters), 188, L59.

Stephens, S.A., 1969, Ph.D. Thesis, University of Bombay (unpublished).

Stephens, S.A., 1970a, Proc. Ind.Acad. Sci. 72, Sec. A, 214. 
Stephens, S.A., 1970b, Acta Phys. Acad. Sci. Hungaricae, 29, Suppl. 2, 733. Stephens, S.A., 1971, Astron. Astrophys., 11, 311.

Stone, E.C., 1973, Proc. 13th Int. Conf. on Cosmic Rays, Denver, 5, 3615.

Strong, A.W., J. Wdowczyk and A.W. Wolfendale, 1973, Proc. 13th Int. Conf. on Cosmic Rays, Denver, 1 , 463.

Suh, P.K., 1971, Astron. Astrophys. 15, 206.

Sullivan, J.D. and R. Cowstk, 1973, Proc. 13th Int. Conf. on Cosmic Rays, Denver, paper no. 358.

Swanenburg, B.N., J.J. Burger and P.A.J. de Korte, 1971, Proc. 12th Int. Conf. on Cosmic Rays, Hobart, $\underline{5}, 1714$.

Syrovatskii, S.I., 1959, Soviet Astron. A.J., 3, 22.

Syrovatskii, S.I., and B.M. Kuzhevsky, 1970, Acta Phys. Acad. Sc1., Hungaricae, 29, Supp1. 1, 313 .

Tandon, S:N., 1970, Ph.D. Thesis, University of Bombay (unpub1ished).

Teegarden, B.J., F.B. McDonald and V.K. Balasubrahmanyan, 1970, Acta Phys. Acad. Sci. Hungaricae, 29, Supp1. 1, 345.

Teegarden, B.J., T.T. von Rosenvinge and F.B. McDonald, 1973a, Astrophys J., 180,571 .

Teegarden, B.J., T.T. von Rosenvinge and F.B. McDonald, 1973b, Proc. 13th Int. Conf. on Cosmic Rays, Denver, paper no. 400.

Toomre, A., 1972, Quart. J. Roy. Astron. Soc. 13, 241.

Truran, J.W. and W.D. Arnett, 1970, Astrophys J. 160, 181.

Truran, J.W. and A.G.W. Cameron, 1971, Astrophys. Sp. Sc1. 14, 179.

Turtle, A.J., J.F. Pugh, S. Kenderdine and I.I.K. Pauliny-Toth, 1962, Mon. Not. Roy. Astron. Soc., 124, 297.

Van de Hulst, H.C., 1958, Rev. Mod. Phys., 30, 913.

Van Woerden, H., 1967, I.A.U. Symposium No. 31, Ed. H. van Woerden (Academic Press), p. 1.

Verma, S.D., 1968, Astrophys. J. 152, 537.

Verma, S.D., 1971, Proc. 12th Int. Conf. on Cosmic Rays, Hobart, 1 , 31. 
Verma, R.P., T.N. Rengarajan, S.N. Tandon, S.V. Damle and Y. Pal, 1972, Nature Phys. Sc1. 240, 135.

Verschuur, G.L., 1969", Nature, 223, 140.

Von Rosenvinge, J.J., W.R. Webber and J.F. Ormes, 1969b, Astrophys. Sp. Sci., $\underline{3}, 4$.

Von Rosenvinge, J.J., W.R. Webber, and J.F. Ormes, 1969c, Astrophys. Sp. Sci., $\underline{5}, 342$.

Voshage, H. and H. Hinterberger, 1963, Radio Active Dating (Vienna, Int. Atomic Energy Agency), p. 367.

Waddington, C.J., 1969, Astrophys. Sp. Sci., 5,3 .

Walraven, T., A.B. Muller, P.T. Oosterhoff, 1958, Bu11. Astr. Inst. Neth., 14,81 .

Wayland, J. and T. Bowen, 1968, Phys. Rev. 171, 1376.

Webbink, R.F. and W.0. Jeffers, 1969, Sp. Sc1. Rev., 10, 191.

Webber, W.R., 1968, Aust. J. Phys. 21, 845.

Webber, W.R. and J.F. Ormes, 1967, J. Geophys. Res., 72, 5957.

Webber, W.R. and J.M. Rockstroh, 1973, J. Geophys. Res. 78, 1.

Webber, W.R., S.V. Damle and J. Kish, 1972, Astrophys. Sp. Sci. 15, 245.

Webber, W.R., J.A. Leznlah, J. Kish and S.V. Damle, 1973a, Astrophys. Sp. Sci. 24,17 .

Webber, W.R., J.A. Leznlah, J.C. Kish and S.V. Damle, 1973b, Nature Phys. Sci. 241, 96 .

Webber, W.R., J.A. Lezniah, J. Kish, 1973c, Proc. 13th Int. Conf. on Cosmic Rays, Denver, 1 , 248.

Webber, W.R., J.A. Lezniah, and S.V. Damle, 1973d, J. Geophys. Res. 78, 1487.

Webster, A.S., 1970, Astrophys. Letters, $\underline{5}, 189$.

Webster, A.S., 1974, Mon. Not. Roy. Astron. Soc. 166, 355.

Wentze1, D.G., 1969, Astrophys. J., 156, 303.

Wentzel, D.G., 1971, Astrophys. J. 163, 503.

Wentze1, D.G., 1974, Ann. Rev. Astron. Astrophys, 12, 71.

Westerhout, G., 1957, Bu11. Astron. Inst. Neth: 13, 201. 
Westerhout, G., 1958, Bull. Astron. Inst. Neth., 14, 215.

Wielebinski, R., D.H. Smith and X.G. Cardenas, 1968, Aust. J. Phys. 21, 185 .

Williamsen, K.D., A.G..Blalr, L.L. Cartlin,.R.D. Kiebert, E.G. Loyd and A.V. Romero, 1973, Nature Phys. Sci. 241, 79.

W11son, R.W. and J.G. Bolton, 1960, Pub1. Astron. Soc. Pacif1c, 72, 331.

Wolfendale, A.W., 1969, Proc. 11th Int. Conf. on Cosmic Rays, Budapest, Ed. G. Bozoki, E. Gombost, A. Sebestyen and A. Somogyi, p. 567.

Wooley, R., 1965, in "Galactic Structure", Ed., A. Blaauw and M. Schmidt, University of Chicago Press, p. 85.

Yash Pa1, 1969, Proc. 11th Int. Conf. on Cosmic Rays, Budapest, Invited Papers and Rapporteur talks, p. 241.

Yates, K.W. and R. Wielebinski, 1966, Aust. J. Phys., 19, 389.

Zatsepin, V.I., 1971, Proc. 12th Int. Conf. on Cosmic Rays, Hobart, 5 , 1720.

Zatsepin, G.J. and U.K. Kuzm1n, 1962, Sov. Phys. JETP 14, 1294.

Zimmermann, H., 1964, Astron. Nachor. 288, 95. 